\title{
Multicomponent cascade reaction by metal-free aerobic oxidation for synthesis of highly functionalized 2-amino-4-cumarinyl-5-arylpyrroles
}

Quan-Xing Zi, Chang-Long Yang, Kun Li, Qin Luo, Jun Lin* and Sheng-Jiao Yan*

Key Laboratory of Medicinal Chemistry for Natural Resource (Yunnan University), Ministry of Education, School of Chemical Science and Technology, Yunnan University, Kunming, 650091, P. R. China.

\section{Supporting Information}

Table of Contents

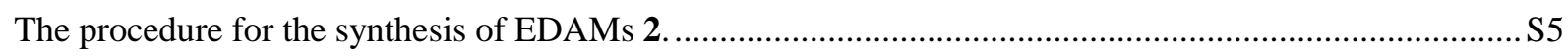

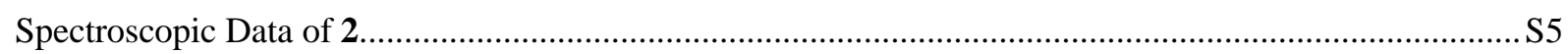

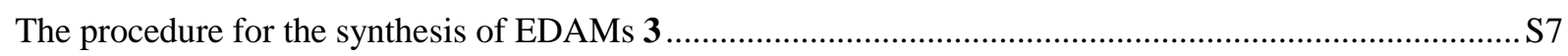

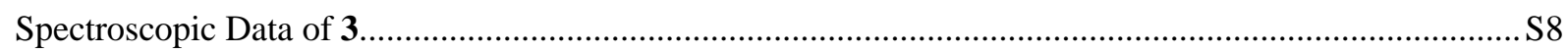

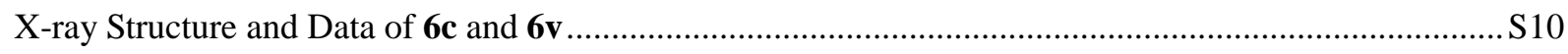

Figure S1. X-Ray crystal structure of $\mathbf{6 c}$, ellipsoids are drawn at the $30 \%$ probability level.....................S10

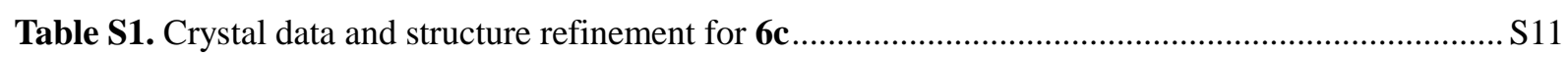

Figure S2. X-Ray crystal structure of $\mathbf{6 v}$, ellipsoids are drawn at the 30\% probability level.....................S12

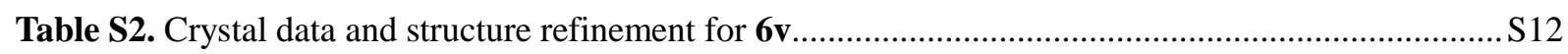

Figure S3. ${ }^{1} \mathrm{H}$ NMR $\left(600 \mathrm{MHz}, \mathrm{DMSO}-d_{6}\right)$ spectra of compound 2a.................................................... 13

Figure S4. ${ }^{13} \mathrm{C}\left\{{ }^{1} \mathrm{H}\right\}$ NMR $\left(150 \mathrm{MHz}\right.$, DMSO- $\left.d_{6}\right)$ spectra of compound 2a............................................ 14

Figure S5. ${ }^{1} \mathrm{H}$ NMR (600 MHz, DMSO- $d_{6}$ ) spectra of compound 2b.................................................. 15

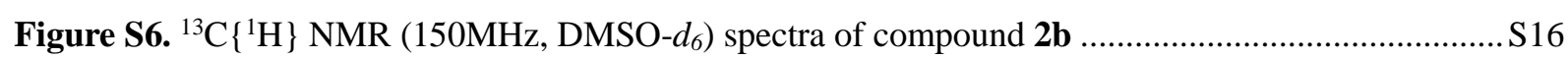

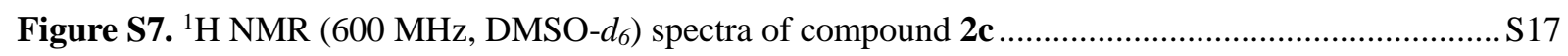




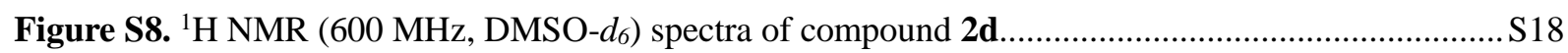

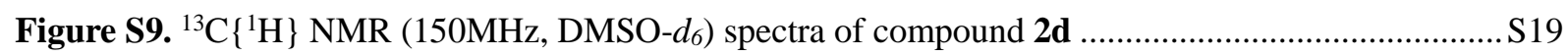

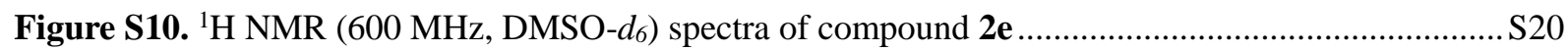

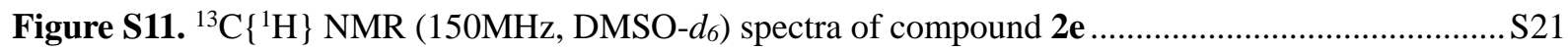

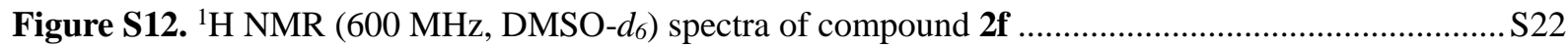

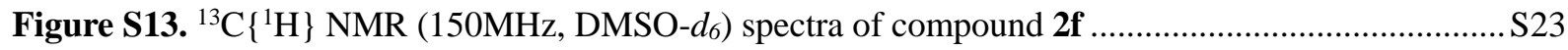

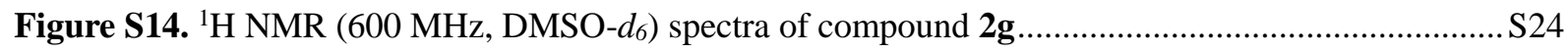

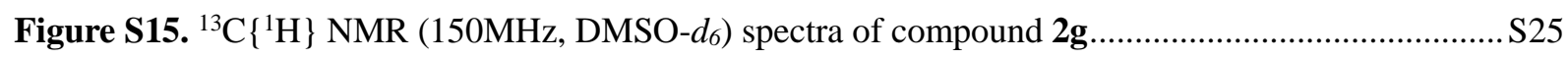

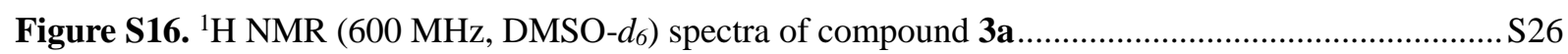

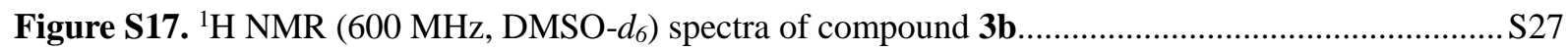

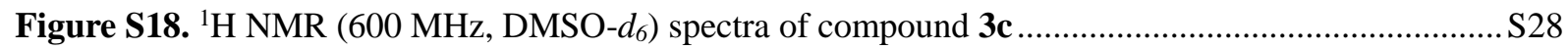

Figure S19. ${ }^{1} \mathrm{H}$ NMR (600 MHz, DMSO- $\left.d_{6}\right)$ spectra of compound 3d................................................. 29

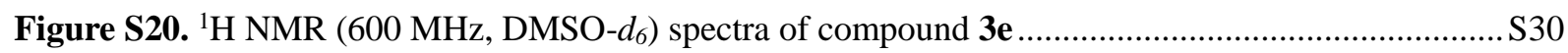

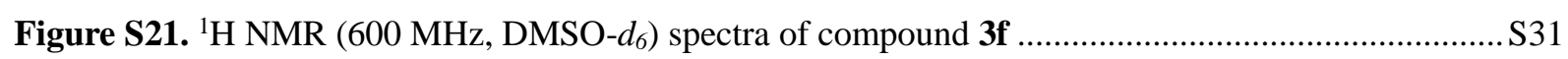

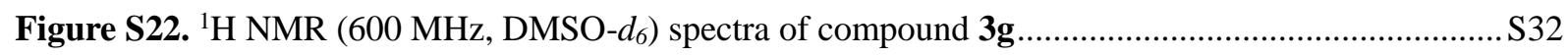

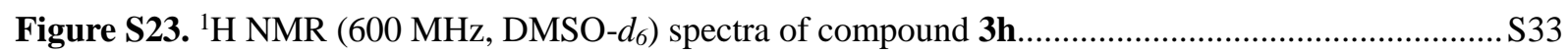

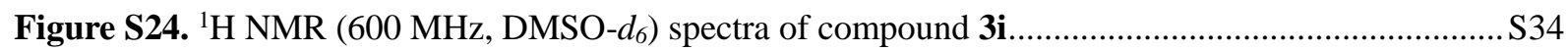

Figure S25. ${ }^{1} \mathrm{H}$ NMR (600 MHz, DMSO- $d_{6}$ ) spectra of compound 5a

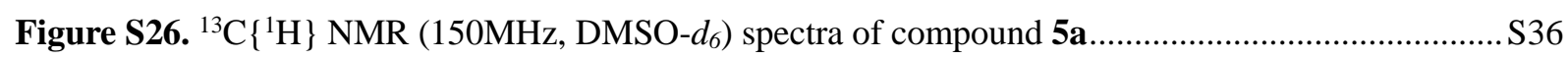

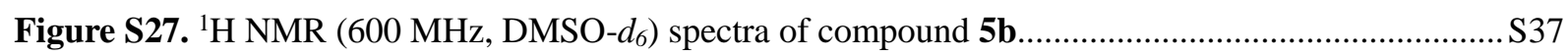

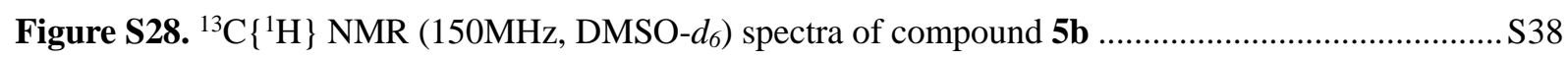

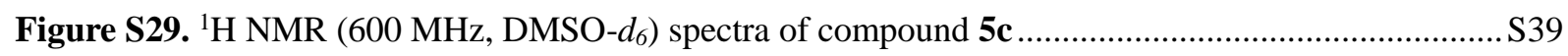

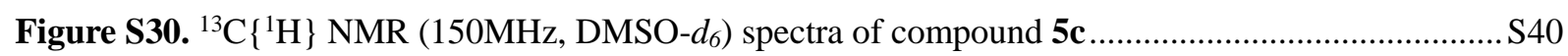

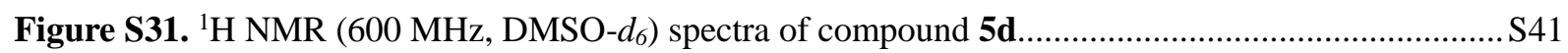

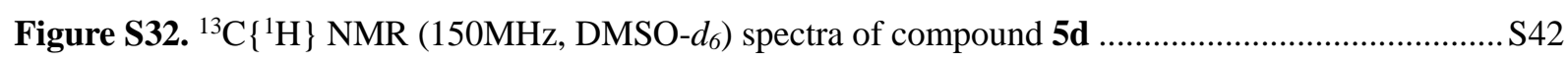

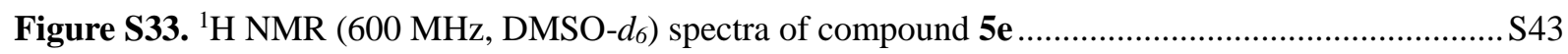

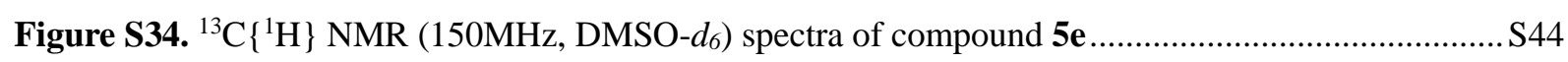

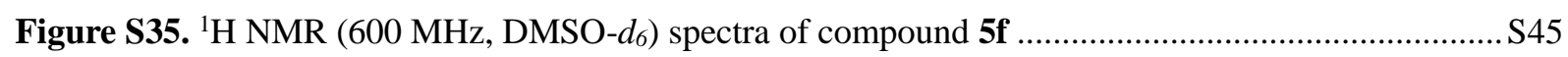

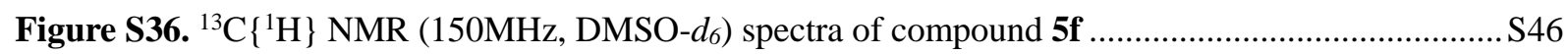

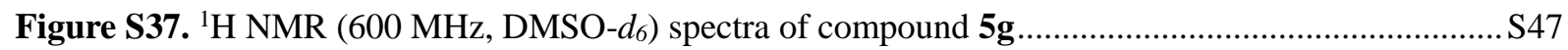

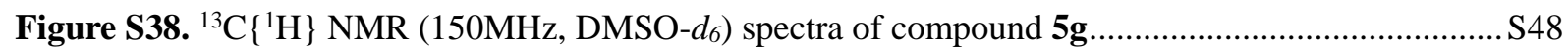

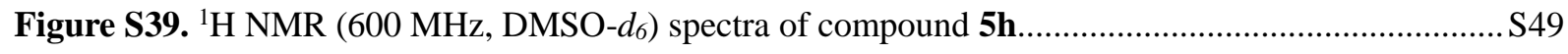

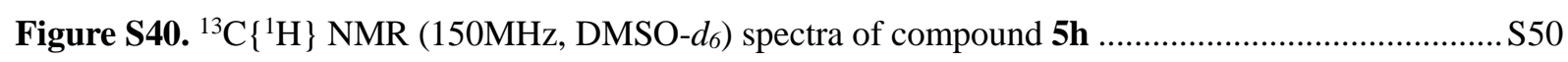

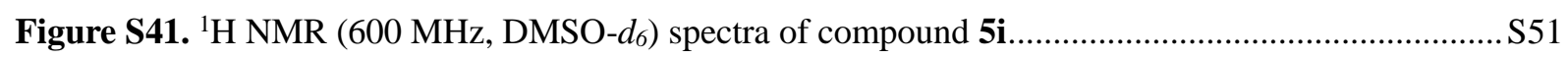

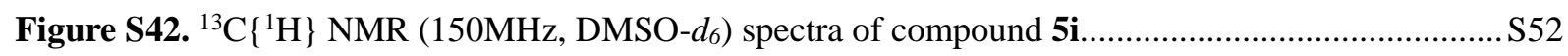

Figure S43. ${ }^{1} \mathrm{H}$ NMR (600 MHz, DMSO- $\left.d_{6}\right)$ spectra of compound $\mathbf{5 j}$................................................ 553

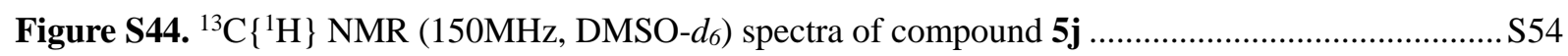


Figure S45. ${ }^{1} \mathrm{H}$ NMR (600 MHz, DMSO- $\left.d_{6}\right)$ spectra of compound 5k. S55

Figure S46. ${ }^{13} \mathrm{C}\left\{{ }^{1} \mathrm{H}\right\}$ NMR $\left(150 \mathrm{MHz}, \mathrm{DMSO}-d_{6}\right)$ spectra of compound $\mathbf{5 k}$ S56

Figure S47. ${ }^{1} \mathrm{H}$ NMR $\left(600 \mathrm{MHz}, \mathrm{DMSO}-d_{6}\right)$ spectra of compound $6 \mathbf{a}$ S57

Figure S48. ${ }^{13} \mathrm{C}\left\{{ }^{1} \mathrm{H}\right\}$ NMR (150 MHz, DMSO-d $d_{6}$ ) spectra of compound $6 \mathbf{a}$ S58

Figure S49. ${ }^{1} \mathrm{H}$ NMR $\left(500 \mathrm{MHz}, \mathrm{DMSO}-d_{6}\right)$ spectra of compound $\mathbf{6 b}$ S59

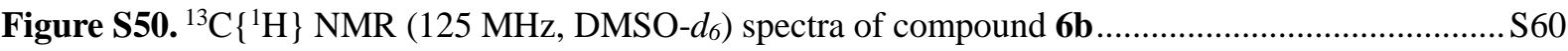

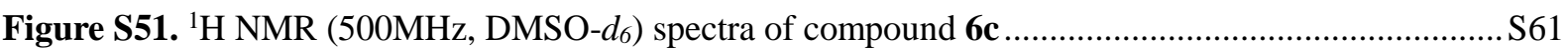

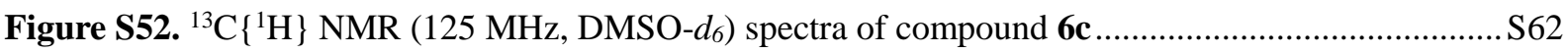

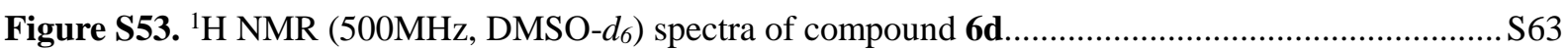

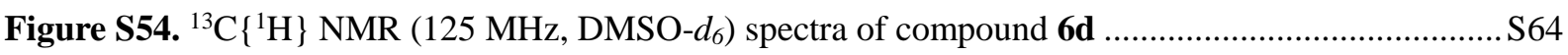

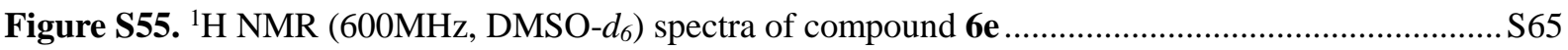

Figure S56. ${ }^{13} \mathrm{C}\left\{{ }^{1} \mathrm{H}\right\}$ NMR (150 MHz, DMSO-d $d_{6}$ spectra of compound 6e....................................S66

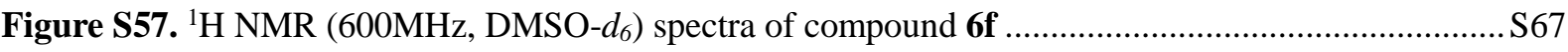

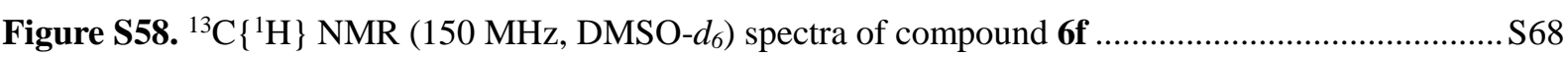

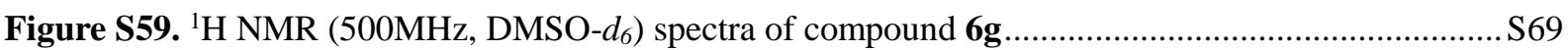

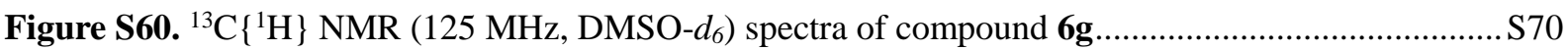

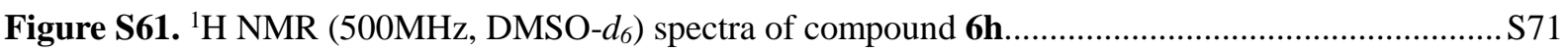

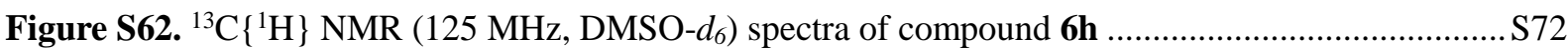

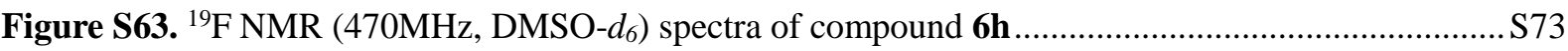

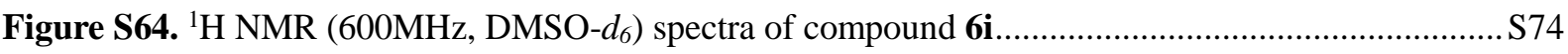

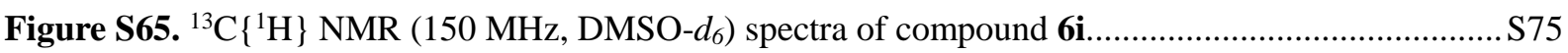

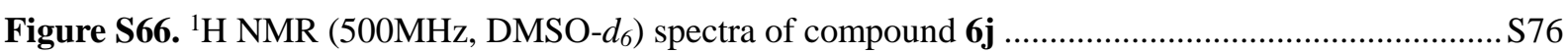

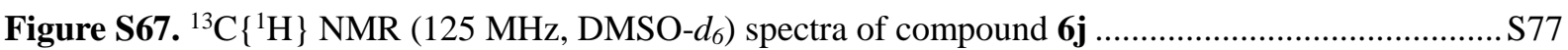

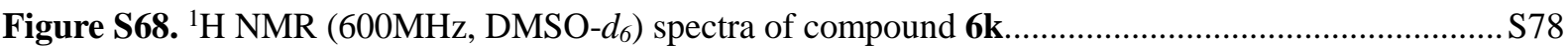

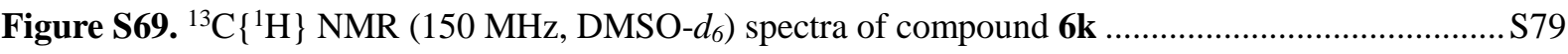

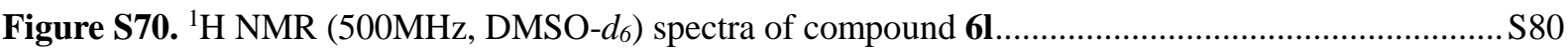

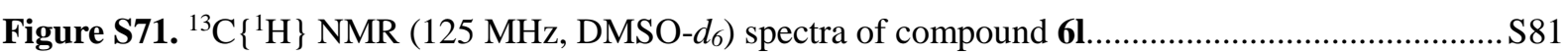

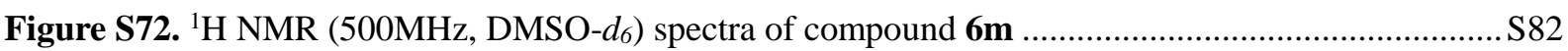

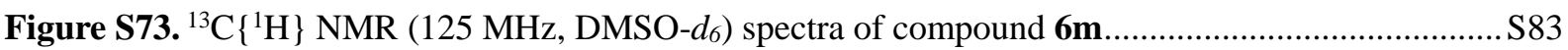

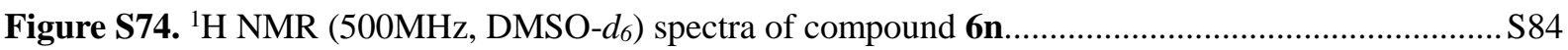

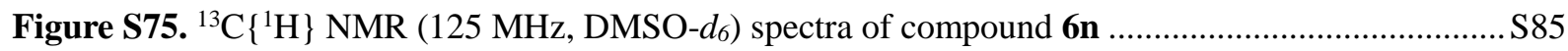

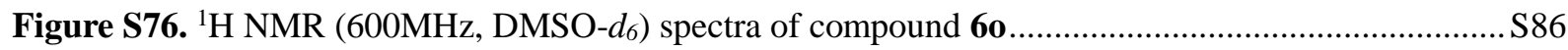

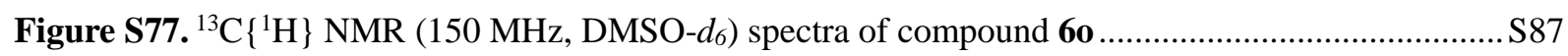

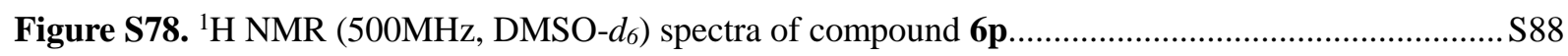

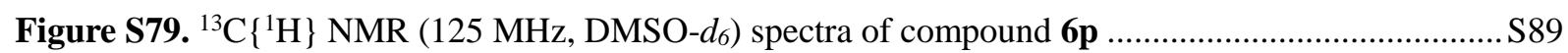

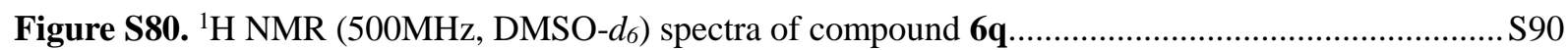

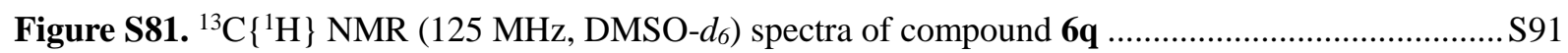


Figure S82. ${ }^{1} \mathrm{H}$ NMR $\left(600 \mathrm{MHz}\right.$, DMSO- $\left.d_{6}\right)$ spectra of compound $\mathbf{6 r}$ S92

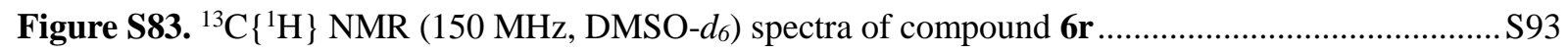

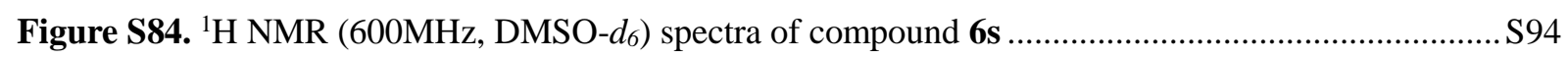

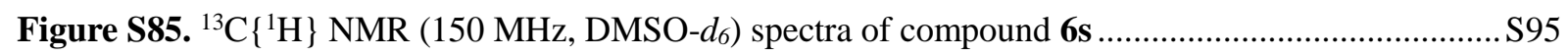

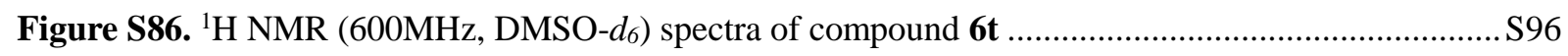

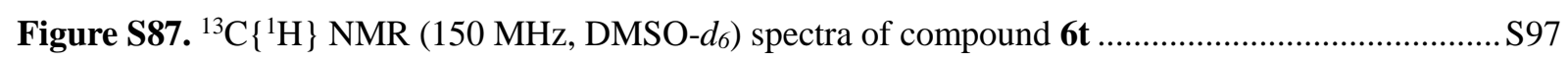

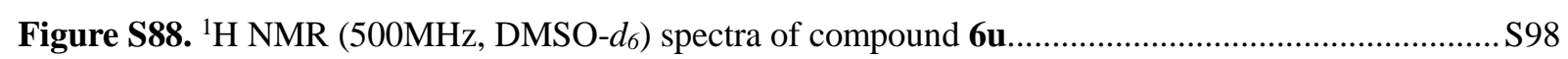

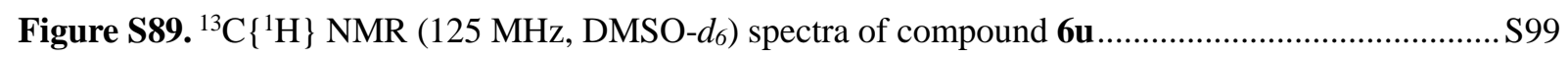

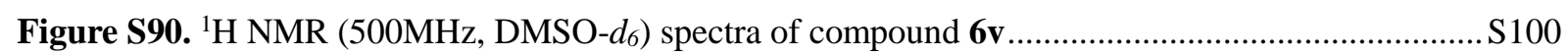

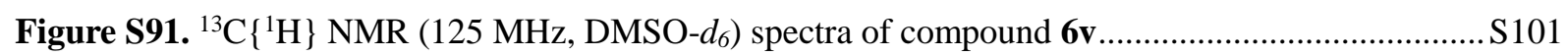

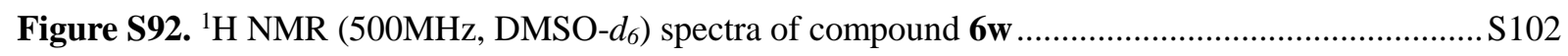

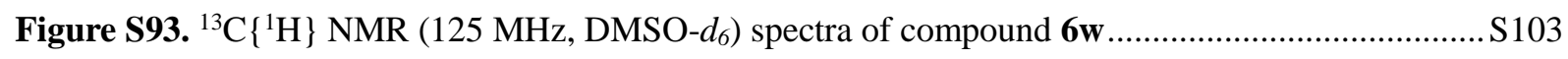

Figure S94. ${ }^{1} \mathrm{H}$ NMR $\left(500 \mathrm{MHz}\right.$, DMSO-d $\left.d_{6}\right)$ spectra of compound 7r ............................................ S104

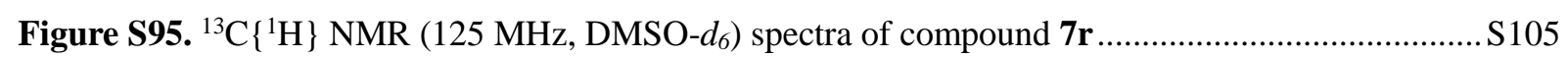

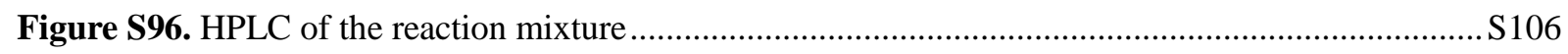

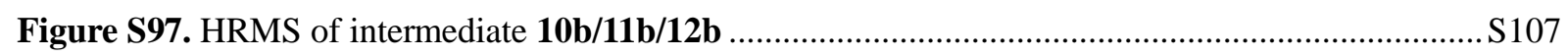

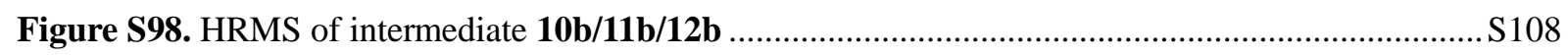

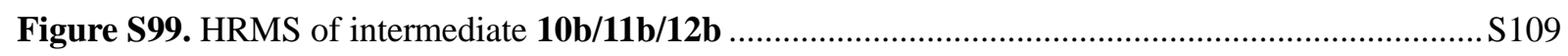

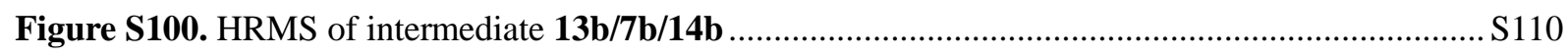

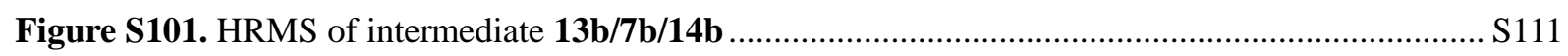

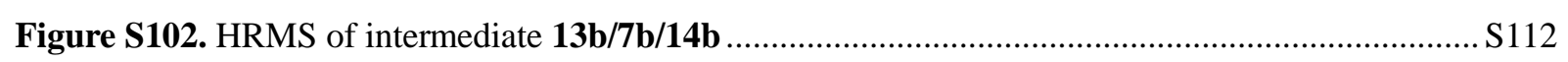

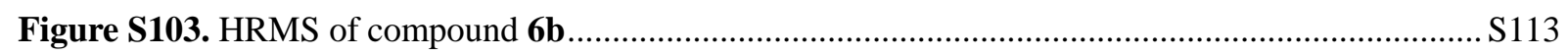

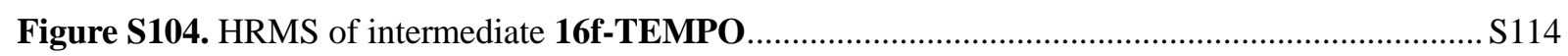

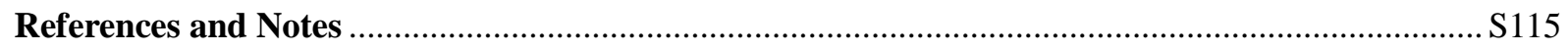




\section{The procedure for the synthesis of EDAMs 2.}

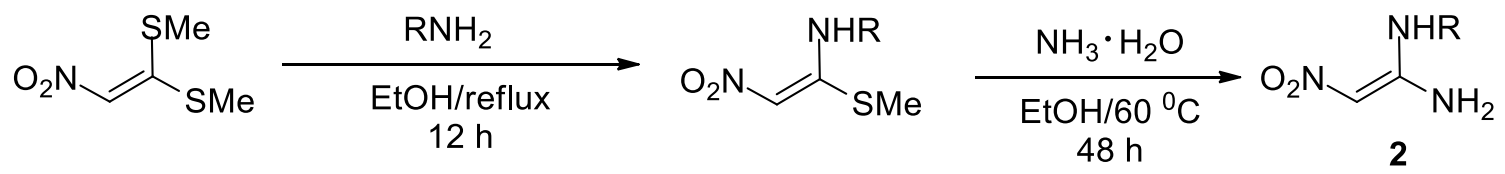

EDAMs (2) were prepared according to the literature. ${ }^{1}$ The mixture of ketene dithioacetals (15 mmol) and alkylamine or arylamine $(15 \mathrm{mmol})$ in ethanol $(25 \mathrm{~mL})$ was stirred for about 12 hours at reflux. The mixture was cooled to room temperature and the product collected by filtration as a withe solid with $80 \sim 90 \%$. Then, this white solid $(10 \mathrm{mmol})$, ethanol $(15 \mathrm{~mL})$ and $\mathrm{NH}_{3} \cdot \mathrm{H}_{2} \mathrm{O}(50 \mathrm{~mL})$ was changed in a round-bottom flask. The mixture was stirred for about 48 hours at $60{ }^{\circ} \mathrm{C}$. The mixture was cooled to room temperature and the product collected by filtration as a solid with $82 \sim 88 \%$.

\section{Spectroscopic Data of 2}

\section{$N$-(4-Methoxyphenethyl)-2-nitroethene-1,1-diamine (2a)}<smiles>COc1ccc(CCN/C(N)=C/[N+](=O)[O-])cc1</smiles>

Yellow solid; Mp: $162-163{ }^{\circ} \mathrm{C}$; IR (KBr): 3472, 1599, 1461, 1130, 804, $766 \mathrm{~cm}^{-1}$; ${ }^{1} \mathrm{H}$ NMR (600MHz, DMSO-d $): \delta=2.76-2.79$ (m, 2H, $\mathrm{ArCH}_{2}$ ), 3.36-3.41 (m, 2H, $\mathrm{NHCH}_{2}$ ), 3.73 (s, $\left.3 \mathrm{H}, \mathrm{OCH}_{3}\right), 6.35$ (s, 1H, CH), 6.87-6.88 (m, 2H, ArH), 7.19-7.20 (m, 2H, ArH), 7.78 (br, 2H, $\mathrm{NH}_{2}$ ), 9.22 (br, 1H, NH); ${ }^{13} \mathrm{C}$ NMR (150 MHz, DMSO-d6): $\delta=33.9,42.7,55.5,97.7,114.3$, 130.2, 130.8, 157.7, 158.4; HRMS (TOF ES ${ }^{+}$): $m / z$ calcd for $\mathrm{C}_{11} \mathrm{H}_{16} \mathrm{~N}_{3} \mathrm{O}_{3}[\mathrm{M}+\mathrm{H}]^{+}, 238.1186$; found, 238.1193.

\section{$N$-(4-Methylphenethyl)-2-nitroethene-1,1-diamine (2b)}<smiles>Cc1ccc(CCN/C(N)=C/[N+](=O)[O-])cc1</smiles>

White solid; Mp: $150-151{ }^{\circ} \mathrm{C}$; IR (KBr): 3321, 1614, 1453, 1139, 832, $736 \mathrm{~cm}^{-1} ;{ }^{1} \mathrm{H}$ NMR (600MHz, DMSO-d $): \delta=2.28\left(\mathrm{~s}, 3 \mathrm{H}, \mathrm{CH}_{3}\right), 2.78-2.81\left(\mathrm{~m}, 2 \mathrm{H}, \mathrm{ArCH}_{2}\right), 3.36-3.45(\mathrm{~m}, 2 \mathrm{H}$, $\mathrm{NHCH}_{2}$ ), 6.36 (s, 1H, CH), 7.12-7.17 (m, 4H, ArH), 7.77 (br, 2H, NH 2$), 9.21$ (br, 1H, NH); 
${ }^{13} \mathrm{C}$ NMR (150 MHz, DMSO- $\left.d_{6}\right): \delta=21.1,34.3,42.5,99.1,129.1,129.4,135.8,135.6,157.7$;

HRMS (TOF ES ${ }^{+}$): $m / z$ calcd for $\mathrm{C}_{11} \mathrm{H}_{15} \mathrm{~N}_{3} \mathrm{~N}_{\mathrm{a}} \mathrm{O}_{2}$ [M+Na] $]^{+}$, 244.1056; found, 244.1061.

\section{2-Nitro- $N$-phenethylethene-1,1-diamine $(2 \mathrm{c})^{1 \mathrm{a}}$}

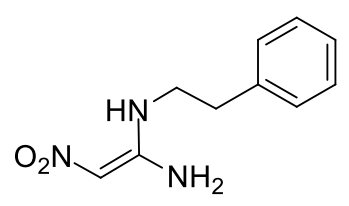

White solid; Mp: $147-148{ }^{\circ} \mathrm{C}$ [Lit. $148-149{ }^{\circ} \mathrm{C}$ ]; IR (KBr): 3197, 2362, 1656, 1354, 894, 760 $\mathrm{cm}^{-1} ;{ }^{1} \mathrm{H}$ NMR $\left(600 \mathrm{MHz}, \mathrm{DMSO}-d_{6}\right): \delta=2.83-2.86\left(\mathrm{~m}, 2 \mathrm{H}, \mathrm{ArCH}_{2}\right), 3.44-3.46(\mathrm{~m}, 2 \mathrm{H}$, $\mathrm{NHCH}_{2}$ ), 6.35 (s, 1H, CH), 7.23-7.33 (m, 4H, ArH), 7.81(br, 2H, NH 2$), 9.21$ (br, 1H, NH); HRMS (TOF ES ${ }^{+}$): $m / z$ calcd for $\mathrm{C}_{10} \mathrm{H}_{14} \mathrm{~N}_{3} \mathrm{O}_{2}[\mathrm{M}+\mathrm{H}]^{+}, 208.1081$; found, 208.1086.

\section{$N$-(4-Chlorophenethyl)-2-nitroethene-1,1-diamine (2d)}

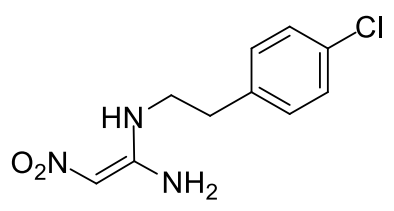

White solid; Mp: $143-144{ }^{\circ} \mathrm{C}$; IR (KBr): 3020, 2733, 1688, 1441, 1141, 939, $826 \mathrm{~cm}^{-1}$; ${ }^{1} \mathrm{H}$ NMR (600MHz, DMSO- $\left.d_{6}\right): \delta=2.83-2.85\left(\mathrm{~m}, 2 \mathrm{H}, \mathrm{ArCH}_{2}\right), 3.35-3.45\left(\mathrm{~m}, 2 \mathrm{H}, \mathrm{NHCH}_{2}\right)$, 6.35 (s, 1H, CH), 7.31-7.32 (m, 2H, ArH), 7.37-7.38 (m, 2H, ArH), 7.80 (br, 2H, NH $), 9.19$ (br, 1H, NH); ${ }^{13} \mathrm{C}$ NMR (150 MHz, DMSO-d $): \delta=34.0,42.2,99.1,128.8,131.2,131.6$, 138.0, 157.7; HRMS (TOF ES ${ }^{+}$): $m / z$ calcd for $\mathrm{C}_{10} \mathrm{H}_{12} \mathrm{ClN}_{3} \mathrm{O}_{2}[\mathrm{M}+\mathrm{H}]^{+}, 242.0691$; found, 242.0698 .

$N$-(4-Fluorophenethyl)-2-nitroethene-1,1-diamine (2e)

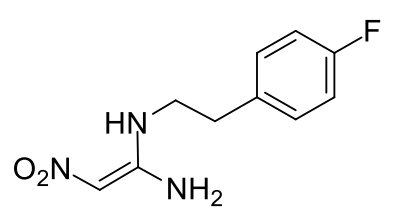

Yellow solid; Mp: $140-141^{\circ} \mathrm{C}$; IR (KBr): 3428, 2370, 1598, 1151, 873, $789 \mathrm{~cm}^{-1}$; ${ }^{1} \mathrm{H}$ NMR (600MHz, DMSO-d $): \delta=2.86-2.88\left(\mathrm{~m}, 2 \mathrm{H}, \mathrm{ArCH}_{2}\right), 3.46 \sim 3.49\left(\mathrm{~m}, 2 \mathrm{H}, \mathrm{NHCH}_{2}\right), 6.37$ (s, 1H, CH), 7.04-7.16 (m, 3H, ArH), 7.34-7.38 (m, 1H, ArH), 7.82 (br, 2H, NH2), 9.28 (br, 1H, $\mathrm{NH}) ;{ }^{13} \mathrm{C}$ NMR $\left(150 \mathrm{MHz}, \mathrm{DMSO}-d_{6}\right): \delta=34.4,42.1,99.1,113.7$ (d, $\left.J=21.0 \mathrm{~Hz}\right), 116.0$ (d, $J=21.0 \mathrm{~Hz}), 125.4,130.7(\mathrm{~d}, J=7.5 \mathrm{~Hz}), 141.9(\mathrm{~d}, J=7.5 \mathrm{~Hz}), 157.7,162.7(\mathrm{~d}, J=241.5$ $\mathrm{Hz}$ ); HRMS (TOF $\mathrm{ES}^{+}$): $m / z$ calcd for $\mathrm{C}_{10} \mathrm{H}_{12} \mathrm{ClN}_{3} \mathrm{O}_{2}[\mathrm{M}+\mathrm{H}]^{+}, 242.0691$; found, 242.0698 . 


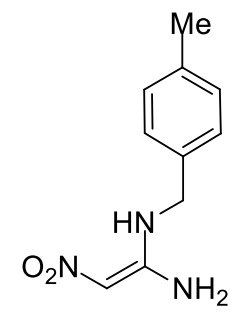

Yellow solid; Mp: $163-164^{\circ} \mathrm{C}$; IR (KBr): 3410, 1533, 1171, 807, $684 \mathrm{~cm}^{-1} ;{ }^{1} \mathrm{H}$ NMR $\left(600 \mathrm{MHz}, \mathrm{DMSO}-d_{6}\right): \delta=2.30\left(\mathrm{~s}, 3 \mathrm{H}, \mathrm{CH}_{3}\right), 4.40-4.41\left(\mathrm{~m}, 2 \mathrm{H}, \mathrm{CH}_{2}\right), 6.42(\mathrm{~s}, 1 \mathrm{H}, \mathrm{CH})$, 7.19-7.22 (m, 4H, ArH), 7.91 (br, 2H, $\left.\mathrm{NH}_{2}\right), 9.41$ (br, $\left.1 \mathrm{H}, \mathrm{NH}\right) ;{ }^{13} \mathrm{C}$ NMR $(150 \mathrm{MHz}$, DMSO- $\left.d_{6}\right): \delta=21.1,44.3,99.2,127.8,129.6,134.5,137.2,157.8$. HRMS $\left(\mathrm{TOF} \mathrm{ES}^{+}\right): \mathrm{m} / \mathrm{z}$ calcd for $\mathrm{C}_{10} \mathrm{H}_{13} \mathrm{~N}_{3} \mathrm{O}_{2}[\mathrm{M}+\mathrm{H}]^{+}$, 208.1081; found, 208.1084.

\section{2-Nitro- $N$-(p-tolyl)ethene-1,1-diamine (2g)}<smiles>Cc1ccc(N/C(N)=C/[N+](=O)[O-])cc1</smiles>

Yellow solid; Mp: $185-186^{\circ} \mathrm{C}$; IR (KBr): 3278, 1633, 1435, 963, $879 \mathrm{~cm}^{-1} ;{ }^{1} \mathrm{H}$ NMR $\left(600 \mathrm{MHz}, \mathrm{DMSO}-d_{6}\right): \delta=2.33\left(\mathrm{~s}, 3 \mathrm{H}, \mathrm{CH}_{3}\right), 6.46(\mathrm{~s}, 1 \mathrm{H}, \mathrm{CH}), 7.15-7.16(\mathrm{~m}, 2 \mathrm{H}, \mathrm{ArH})$, 7.26-7.27 (m, 2H, ArH), 7.61 (br, $\left.1 \mathrm{H}, \mathrm{NH}_{2}\right), 10.01$ (br, $\left.1 \mathrm{H}, \mathrm{NH}\right) ;{ }^{13} \mathrm{C}$ NMR $(150 \mathrm{MHz}$, DMSO- $d_{6}$ ): $\delta=21.0,99.6,125.1,130.6,133.4,136.4,156.7$; HRMS (TOF ES ${ }^{+}$): $m / z$ calcd for $\mathrm{C}_{10} \mathrm{H}_{13} \mathrm{~N}_{3} \mathrm{O}_{2}[\mathrm{M}+\mathrm{H}]^{+}$, 208.1081; found, 208.1084.

\section{The procedure for the synthesis of EDAMs 3}

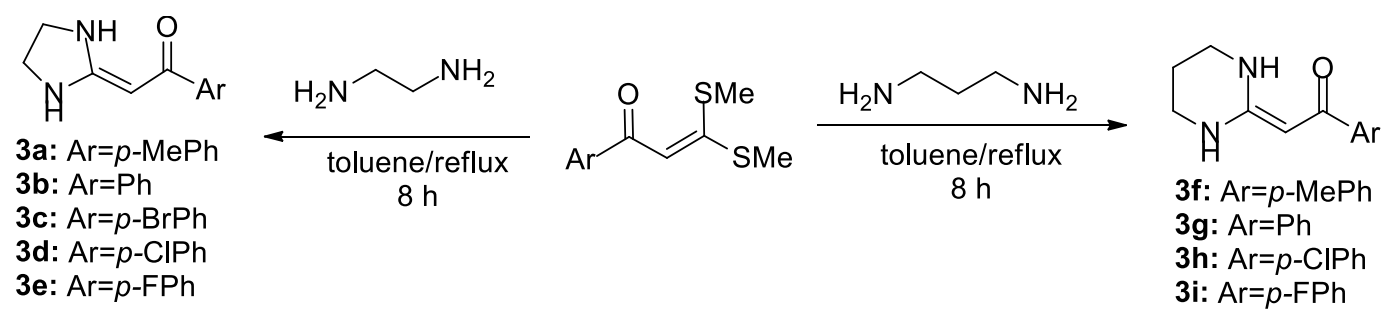

EDAMs (3) were prepared according to the literature. ${ }^{2 a}$ The mixture of ketene dithioacetals (10 mmol) and 1,2-diaminoethane or propane-1,3-diamine (12 mmol) in anhydrous toluene $(40 \mathrm{~mL})$ was stirred for about 8 hours at reflux. The mixture was cooled to room temperature and the product collected by filtration as a withe or yellow solid with $85 \sim 92 \%$. 


\section{Spectroscopic Data of 3}

2-(Imidazolidin-2-ylidene)-1-(p-tolyl)ethanone (3a) $)^{2 b}$

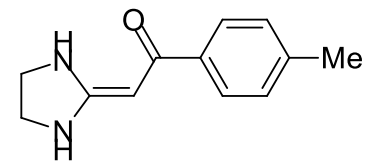

Withe solid (1.82 g, 90\%); Mp: 244-245 ${ }^{\circ} \mathrm{C}$ [Lit. $245{ }^{\circ} \mathrm{C}$ ]; ${ }^{1} \mathrm{H}$ NMR (600 MHz, DMSO- $\left.d_{6}\right): \delta$ $=9.26(\mathrm{~s}, 1 \mathrm{H}, \mathrm{NH}), 7.61(\mathrm{~d}, J=8.0 \mathrm{~Hz}, 2 \mathrm{H}, \mathrm{ArH}), 7.33(\mathrm{~s}, 1 \mathrm{H}, \mathrm{NH}), 7.16(\mathrm{~d}, J=8.0 \mathrm{~Hz}, 2 \mathrm{H}$, $\mathrm{ArH}), 5.24$ (s, 1H, CH), 3.59 (d, $\left.J=7.6 \mathrm{~Hz}, 2 \mathrm{H}, \mathrm{CH}_{2}\right), 3.43\left(\mathrm{~d}, J=7.6 \mathrm{~Hz}, 2 \mathrm{H}, \mathrm{CH}_{2}\right), 2.31$ (s, $3 \mathrm{H}, \mathrm{CH}_{3}$ ); HRMS (TOF ES ${ }^{+}$): $\mathrm{m} / z$ calcd for $\mathrm{C}_{12} \mathrm{H}_{14} \mathrm{~N}_{2} \mathrm{O}[\mathrm{M}+\mathrm{H}]^{+}, 203.1179$; found, 203.1176.

\section{2-(Imidazolidin-2-ylidene)-1-phenylethan-1-one (3b) ${ }^{2 c}$}

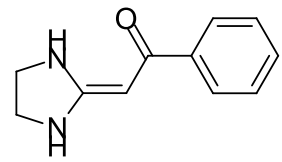

White solid (1.65 g, 88\%); Mp: 212-214 ${ }^{\circ} \mathrm{C}$ [Lit. 208-210 ${ }^{\circ} \mathrm{C}$ ]; ${ }^{1} \mathrm{H}$ NMR $(600 \mathrm{MHz}$, DMSO-d $\left.)_{6}\right): \delta=3.44-3.47\left(\mathrm{~m}, 2 \mathrm{H}, \mathrm{CH}_{2}\right), 3.60-3.63\left(\mathrm{~m}, 2 \mathrm{H}, \mathrm{CH}_{2}\right), 5.27(\mathrm{~s}, 1 \mathrm{H}, \mathrm{CH})$, 7.37-7.38 (m, 4H, ArH), 7.71-7.73 (m, 2H, ArH, NH), 9.29 (br, 1H, NH). HRMS (TOF ES ${ }^{+}$): $m / z$ calcd for $\mathrm{C}_{11} \mathrm{H}_{13} \mathrm{~N}_{2} \mathrm{O}[\mathrm{M}+\mathrm{H}]^{+}, 189.1022$; found, 189.1028 .

\section{1-(4-Bromophenyl)-2-(imidazolidin-2-ylidene)ethanone $(3 c)^{2 b}$}

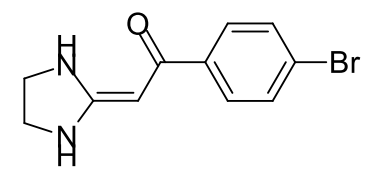

Yellow solid (2.35 g, 88\%); Mp: 272-274 ${ }^{\circ} \mathrm{C}$ [Lit. $275^{\circ} \mathrm{C}$ ]; ${ }^{1} \mathrm{H}$ NMR (600 MHz, DMSO-d 6 ): $\delta=9.25(\mathrm{~s}, 1 \mathrm{H}, \mathrm{NH}), 7.66(\mathrm{~d}, J=8.4 \mathrm{~Hz}, 2 \mathrm{H}, \mathrm{ArH}), 7.56(\mathrm{~d}, J=8.4 \mathrm{~Hz}, 2 \mathrm{H}, \operatorname{ArH}), 7.43$ (s, $1 \mathrm{H}, \mathrm{NH}), 5.24$ (s, 1H, CH), $3.61\left(\mathrm{~d}, J=7.7 \mathrm{~Hz}, 2 \mathrm{H}, \mathrm{CH}_{2}\right), 3.46\left(\mathrm{~d}, J=7.7 \mathrm{~Hz}, 2 \mathrm{H}, \mathrm{CH}_{2}\right)$; HRMS (TOF ES ${ }^{+}$): $\mathrm{m} / z$ calcd for $\mathrm{C}_{11} \mathrm{H}_{12} \mathrm{BrN}_{2} \mathrm{O}[\mathrm{M}+\mathrm{H}]^{+}, 267.0128$; found, 267.0128.

1-(4-Chlorophenyl)-2-(imidazolidin-2-ylidene)ethanone $(3 \mathrm{~d})^{2 \mathrm{~b}}$

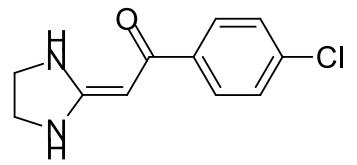

Withe solid (2.05 g, 92\%); Mp: 236-237.5 ${ }^{\circ} \mathrm{C}$ [Lit. $238{ }^{\circ} \mathrm{C}$ ]; ${ }^{1} \mathrm{H}$ NMR (600 MHz, DMSO-d6): $\delta=9.26(\mathrm{~s}, 1 \mathrm{H}, \mathrm{NH}), 7.73(\mathrm{~d}, J=8.5 \mathrm{~Hz}, 2 \mathrm{H}, \mathrm{ArH}), 7.42(\mathrm{~s}, 1 \mathrm{H}, \mathrm{NH}), 7.41(\mathrm{~d}, J=8.5 \mathrm{~Hz}, 2 \mathrm{H}$, $\mathrm{ArH}), 5.25(\mathrm{~s}, 1 \mathrm{H}, \mathrm{CH}), 3.60\left(\mathrm{~d}, J=7.7 \mathrm{~Hz}, 2 \mathrm{H}, \mathrm{CH}_{2}\right), 3.46\left(\mathrm{~d}, J=7.7 \mathrm{~Hz}, 2 \mathrm{H}, \mathrm{CH}_{2}\right)$; HRMS 
(TOF ES ${ }^{+}$): $m / z$ calcd for $\mathrm{C}_{11} \mathrm{H}_{12} \mathrm{ClN}_{2} \mathrm{O}[\mathrm{M}+\mathrm{H}]^{+}, 223.0633$; found, 223.0634.

1-(4-Fluorophenyl)-2-(imidazolidin-2-ylidene)ethanone (3e) $)^{2 d}$

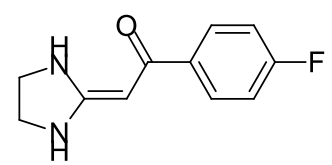

Withe solid (1.86 g, 90\%); Mp: 206-208 ${ }^{\circ} \mathrm{C}$ [Lit. $206{ }^{\circ} \mathrm{C}$ ]; ${ }^{1} \mathrm{H}$ NMR (600 MHz, DMSO- $\left.d_{6}\right): \delta$ $=9.25(\mathrm{~s}, 1 \mathrm{H}, \mathrm{NH}), 7.78-7.76(\mathrm{~m}, 2 \mathrm{H}, \mathrm{ArH}), 7.40(\mathrm{~s}, 1 \mathrm{H}, \mathrm{NH}), 7.17(\mathrm{t}, J=8.8 \mathrm{~Hz}, 2 \mathrm{H}, \mathrm{ArH})$, $5.24(\mathrm{~s}, 1 \mathrm{H}, \mathrm{CH}), 3.61\left(\mathrm{t}, J=8.3 \mathrm{~Hz}, 2 \mathrm{H}, \mathrm{CH}_{2}\right), 3.45$ (t, $J=8.3 \mathrm{~Hz}, 2 \mathrm{H}, \mathrm{CH}_{2}$ ); HRMS (TOF $\mathrm{ES}^{+}$): $m / z$ calcd for $\mathrm{C}_{11} \mathrm{H}_{12} \mathrm{FN}_{2} \mathrm{O}[\mathrm{M}+\mathrm{H}]^{+}, 207.0928$; found, 207.0928.

2-(Tetrahydropyrimidin-2(1H)-ylidene)-1-(p-tolyl)ethan-1-one $(3 f)^{2 c}$

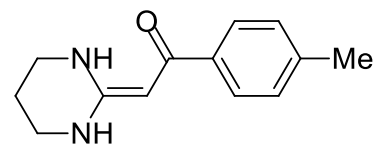

White solid (1.83 g, 85\%); Mp: 248-249 ${ }^{\circ} \mathrm{C}$ [Lit. 248-250 ${ }^{\circ} \mathrm{C}$ ]; ${ }^{1} \mathrm{H}$ NMR $(600 \mathrm{MHz}$, DMSO- $\left.d_{6}\right): \delta=1.82-1.86\left(\mathrm{~m}, 2 \mathrm{H}, \mathrm{CH}_{2}\right), 2.30$ (s, 3H, $\left.\mathrm{CH}_{3}\right), 3.31-3.35\left(\mathrm{~m}, 4 \mathrm{H}, \mathrm{NCH}_{2}, \mathrm{NCH}_{2}\right)$, 5.05 (s, 1H, CH), 7.13-7.15 (m, 2H, ArH), 7.30 (br, 1H, NH), 7.54-7.56 (m, 2H, ArH), 11.16 (br, 1H, NH). HRMS (TOF $\mathrm{ES}^{+}$): $m / z$ calcd for $\mathrm{C}_{13} \mathrm{H}_{17} \mathrm{~N}_{2} \mathrm{O}[\mathrm{M}+\mathrm{H}]^{+}, 217.1335$; found, 217.1339.

Phenyl-2-(tetrahydropyrimidin-2(1H)-ylidene)ethan-1-one $(3 \mathrm{~g})^{2 \mathrm{c}}$

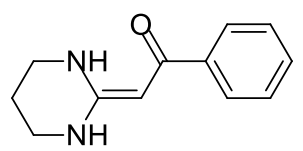

White solid (1.76 g, 87\%); Mp: 210-212 ${ }^{\circ} \mathrm{C}$ [Lit. 206-207 ${ }^{\circ} \mathrm{C}$ ]; ${ }^{1} \mathrm{H}$ NMR $(600 \mathrm{MHz}$, DMSO-d $)): \delta=1.83-1.85\left(\mathrm{~m}, 2 \mathrm{H}, \mathrm{CH}_{2}\right), 3.23-3.35\left(\mathrm{~m}, 4 \mathrm{H}, \mathrm{NCH}_{2}, \mathrm{NCH}_{2}\right), 5.07$ (s, 1H, CH), 7.33-7.35 (m, 4H, ArH), 7.64 7.66 (m, 2H, ArH, NH), 11.14 (br, 1H, NH); HRMS (TOF $\left.\mathrm{ES}^{+}\right): m / z$ calcd for $\mathrm{C}_{12} \mathrm{H}_{14} \mathrm{~N}_{2} \mathrm{O}[\mathrm{M}+\mathrm{H}]^{+}, 203.1179$; found, 203.1183 .

1-(4-Chlorophenyl)-2-(tetrahydropyrimidin-2(1H)-ylidene)ethan-1-one $(3 \mathrm{~h})^{2 \mathrm{c}}$

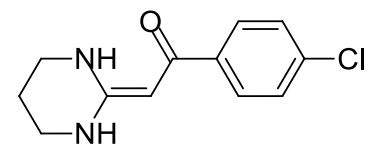

White solid (2.12 g, 90\%); Mp: 213-215 ${ }^{\circ} \mathrm{C}$ [Lit. 213-215 ${ }^{\circ} \mathrm{C}$ ]; ${ }^{1} \mathrm{H}$ NMR $(600 \mathrm{MHz}$, DMSO- $\left.d_{6}\right): \delta=1.83-1.84\left(\mathrm{~m}, 2 \mathrm{H}, \mathrm{CH}_{2}\right), 3.23-3.36\left(\mathrm{~m}, 4 \mathrm{H}, \mathrm{NCH}_{2}, \mathrm{NCH}_{2}\right), 5.06(\mathrm{~s}, 1 \mathrm{H}, \mathrm{CH})$, 
7.39-7.40 (m, 3H, ArH), 7.75-7.66 (m, 2H, ArH, NH), 11.10 (br, 1H, NH); HRMS (TOF ES ${ }^{+}$): $m / z$ calcd for $\mathrm{C}_{12} \mathrm{H}_{14} \mathrm{ClN}_{2} \mathrm{O}[\mathrm{M}+\mathrm{H}]^{+}, 237.0789$; found, 237.0794 .

1-(4-Fluorophenyl)-2-(tetrahydropyrimidin-2(1H)-ylidene)ethan-1-one $(3 i)^{2 c}$

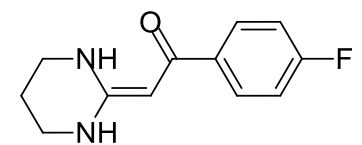

White solid (1.96 g, 89\%); Mp: 223-225 ${ }^{\circ} \mathrm{C}$ [Lit. 228-230 ${ }^{\circ} \mathrm{C}$ ]; ${ }^{1} \mathrm{H}$ NMR $(600 \mathrm{MHz}$, DMSO- $\left.d_{6}\right): \delta=1.83-1.85\left(\mathrm{~m}, 2 \mathrm{H}, \mathrm{CH}_{2}\right), 3.23-3.34\left(\mathrm{~m}, 4 \mathrm{H}, \mathrm{NCH}_{2}, \mathrm{NCH}_{2}\right), 5.03-5.04(\mathrm{~m}, 1 \mathrm{H}$, $\mathrm{CH}), 7.14-7.15$ (m, 2H, ArH), 7.63 (br, 1H, NH), 7.67-7.70 (m, 2H, ArH), 11.10 (br, 1H, NH); HRMS (TOF ES ${ }^{+}$): $m / z$ calcd for $\mathrm{C}_{12} \mathrm{H}_{14} \mathrm{FN}_{2} \mathrm{O}[\mathrm{M}+\mathrm{H}]^{+}, 221.1085$; found, 221.1091.

\section{$\underline{\text { X-ray Structure and Data of } 6 \mathrm{c} \text { and } 6 \mathrm{v}}$}

Single crystal culture and confirmation: First, compound $\mathbf{6 c}$ was added to a bottle and dissolved by the addition of chloroform. Then, the bottle was placed in a jar, and petroleum ether $(15 \mathrm{~mL})$ was added to the bottle. The jar was sealed, and the bottle within the jar remained at room temperature for 4 days. Some crystals appeared, and for single crystal parsing, crystals were selected with sizes of $0.27 \mathrm{~mm}$ x $0.25 \mathrm{~mm}$ x $0.24 \mathrm{~mm}$. The APEX DUO diffractometer was used to obtain single crystal diffraction at $296.15 \mathrm{~K}$ with the use of graphite monochromatic Mo K (lambda $=0.71073 \mathrm{~A}$ ) for diffraction intensity data collection, using $\Phi$ and omega scanning. Multi-scan correction was performed for all intensity data. The crystal structure was solved by the atomic method using the SHELXT program (Supporting Information, Figure S1, CCDC 1916446) $)^{3}$.

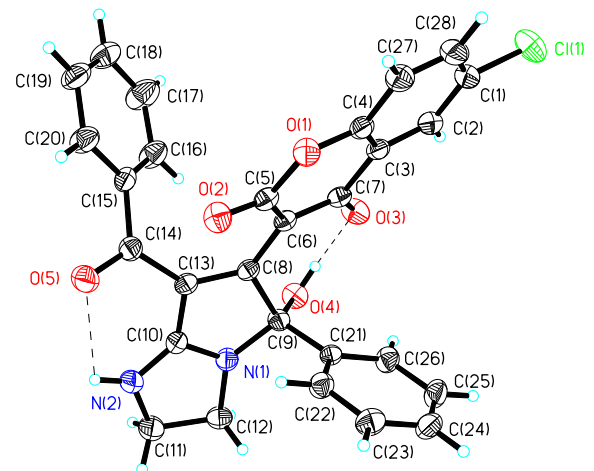

Figure S1. X-Ray crystal structure of $\mathbf{6 c}$, ellipsoids are drawn at the $30 \%$ probability level. 
Table S1. Crystal data and structure refinement for $\mathbf{6 c}$

\begin{tabular}{|c|c|}
\hline Identification code & ysj17 \\
\hline Empirical formula & $\mathrm{C} 31 \mathrm{H} 25 \mathrm{Cl} \mathrm{N} 2 \mathrm{O} 6$ \\
\hline Formula weight & 556.98 \\
\hline Temperature & $296.15 \mathrm{~K}$ \\
\hline Wavelength & $0.71073 \AA$ \\
\hline Crystal system, space group & Triclinic, $\mathrm{P}-1$ \\
\hline Unit cell dimensions & $\begin{array}{ll}\mathrm{a}=9.639(3) \AA & =89.481(4)^{\circ} . \\
\mathrm{b}=11.716(4) \AA, & =78.344(4)^{\circ} \\
\mathrm{c}=13.177(5) \AA . & =70.108(4)^{\circ} .\end{array}$ \\
\hline Volume & $1367.6(8) \AA^{3}$ \\
\hline Z, Calculated density & $2,1.353 \mathrm{Mg} / \mathrm{m}^{3}$ \\
\hline Absorption coefficient & $0.188 \mathrm{~mm}^{-1}$ \\
\hline $\mathrm{F}(000)$ & 580 \\
\hline Theta range for data collection & 1.581 to $27.681^{\circ} .$. \\
\hline Limiting indices & $-12<=\mathrm{h}<=12,-15<=\mathrm{k}<=14,-15<=1<=16$ \\
\hline Reflections collected / unique & $110265861[\mathrm{R}(\mathrm{int})=0.0221]$ \\
\hline Completeness to theta $=25.242^{\circ}$ & $99.5 \%$ \\
\hline Absorption correction & Semi-empirical from equivalents \\
\hline Max. and min. transmission & 0.7456 and 0.6802 \\
\hline Refinement method & Full-matrix least-squares on $\mathrm{F}^{2}$ \\
\hline Data / restraints / parameters & $5861 / 0 / 364$ \\
\hline Goodness-of-fit on $\mathrm{F}^{\wedge} 2$ & 1.019 \\
\hline Final $R$ indices $[\mathrm{I}>2 \operatorname{sigma}(\mathrm{I})]$ & $\mathrm{R} 1=0.0494, \mathrm{wR} 2=0.1063$ \\
\hline $\mathrm{R}$ indices (all data) & $\mathrm{R} 1=0.0897, \mathrm{wR} 2=0.1224$ \\
\hline Extinction coefficient & $\mathrm{n} / \mathrm{a}$ \\
\hline Largest diff. peak and hole & 0.244 and -0.319 e..$\AA^{-3}$ \\
\hline
\end{tabular}

Single crystal culture and confirmation: First, compound $\mathbf{6 v}$ was added to a bottle and dissolved by the addition of chloroform. Then, the bottle was placed in a jar, and petroleum ether $(15 \mathrm{~mL})$ was added to the bottle. The jar was sealed, and the bottle within the jar remained at room temperature for 6 days. Some crystals appeared, and for single crystal parsing, crystals were selected with sizes of $0.24 \mathrm{~mm} \times 0.22 \mathrm{~mm}$ x $0.20 \mathrm{~mm}$. The APEX DUO diffractometer was used to obtain single crystal diffraction at $296 \mathrm{~K}$ with the use of graphite monochromatic Mo K (lambda $=0.71073 \mathrm{~A}$ ) for diffraction intensity data collection, using $\Phi$ and omega scanning. Multi-scan correction was performed for all intensity data. The crystal structure was solved by the atomic method using the SHELXT program (Supporting Information, Figure S1, CCDC 1916449) $)^{3}$. 


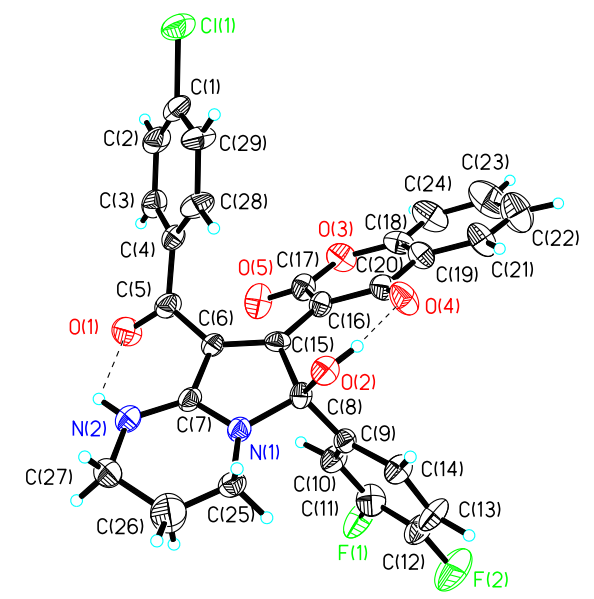

Figure S2. X-Ray crystal structure of $\mathbf{6 v}$, ellipsoids are drawn at the $30 \%$ probability level.

Table S2. Crystal data and structure refinement for $\mathbf{6 v}$

\begin{tabular}{ll}
\hline Identification code & ysj16_sq \\
Empirical formula & $\mathrm{C} 29 \mathrm{H} 19 \mathrm{Cl} \mathrm{F} 2 \mathrm{~N} 2 \mathrm{O} 5$ \\
Formula weight & 548.91 \\
Temperature & $296.15 \mathrm{~K}$ \\
Wavelength & $0.71073 \AA$ \\
Crystal system, space group & $\mathrm{P} 21212 \mathrm{~A}$ \\
Unit cell dimensions & $\mathrm{a}=8.589(2) \AA \quad=90^{\circ}$ \\
& $\mathrm{b}=15.353(4) \AA \quad=90^{\circ}$ \\
& $\mathrm{c}=22.146(6) \AA \quad=90^{\circ}$ \\
Volume & $2920.3(13) \AA^{3}$ \\
Z, Calculated density & $4,1.248 \mathrm{Mg} / \mathrm{m}^{3}$ \\
Absorption coefficient & $0.182 \mathrm{~mm}{ }^{-1}$ \\
F(000) & 1128 \\
Theta range for data collection & 2.268 to $27.693^{\circ}$. \\
Limiting indices & $-11<=\mathrm{h}<=10,-20<=\mathrm{k}<=19,-26<=1<=27$ \\
Reflections collected / unique & $17813 \quad 6609[\mathrm{R}(\mathrm{int})=0.0512]$ \\
Completeness to theta $=25.242^{\circ}$ & $99.8 \%$ \\
Absorption correction & Semi-empirical from equivalents \\
Max. and min. transmission & 0.7456 and 0.7099 \\
Refinement method & Full-matrix least-squares on $\mathrm{F}^{2}$ \\
Data / restraints / parameters & $6609 / 15 / 363$ \\
Goodness-of-fit on F^2 & 0.983 \\
Final R indices [I>2sigma(I)] & $\mathrm{R} 1=0.0530, \mathrm{wR} 2=0.1405$ \\
$\mathrm{R}$ indices (all data) & $\mathrm{R} 1=0.0798, \mathrm{wR} 2=0.1623$ \\
Extinction coefficient & $\mathrm{n} / \mathrm{a}$ \\
Largest diff. peak and hole & 0.257 and -0.309 e. $\mathrm{A}^{\wedge}-3$ \\
\hline
\end{tabular}




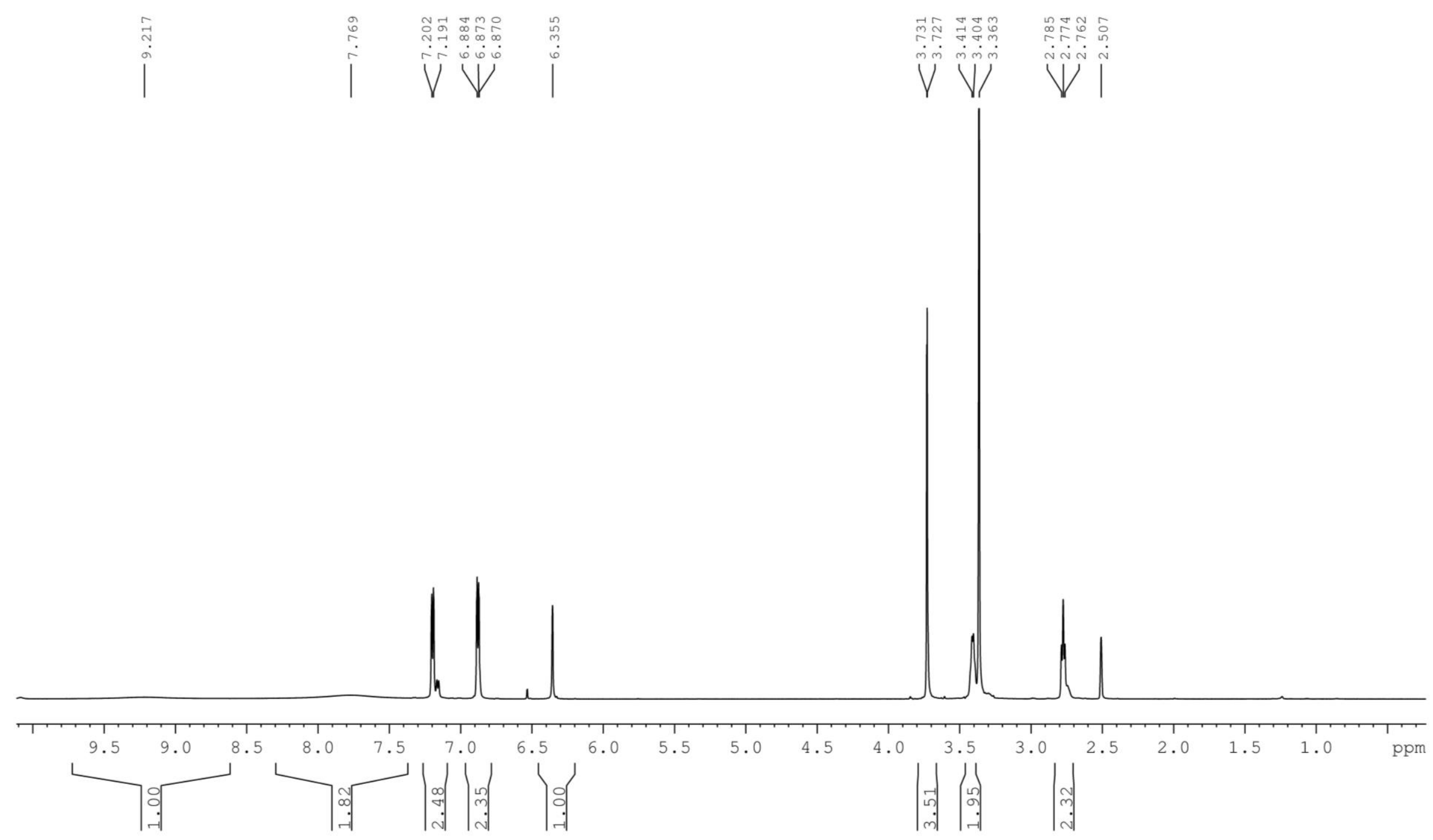

Figure S3. ${ }^{1} \mathrm{H}$ NMR (600 MHz, DMSO- $\left.d_{6}\right)$ spectra of compound 2a 


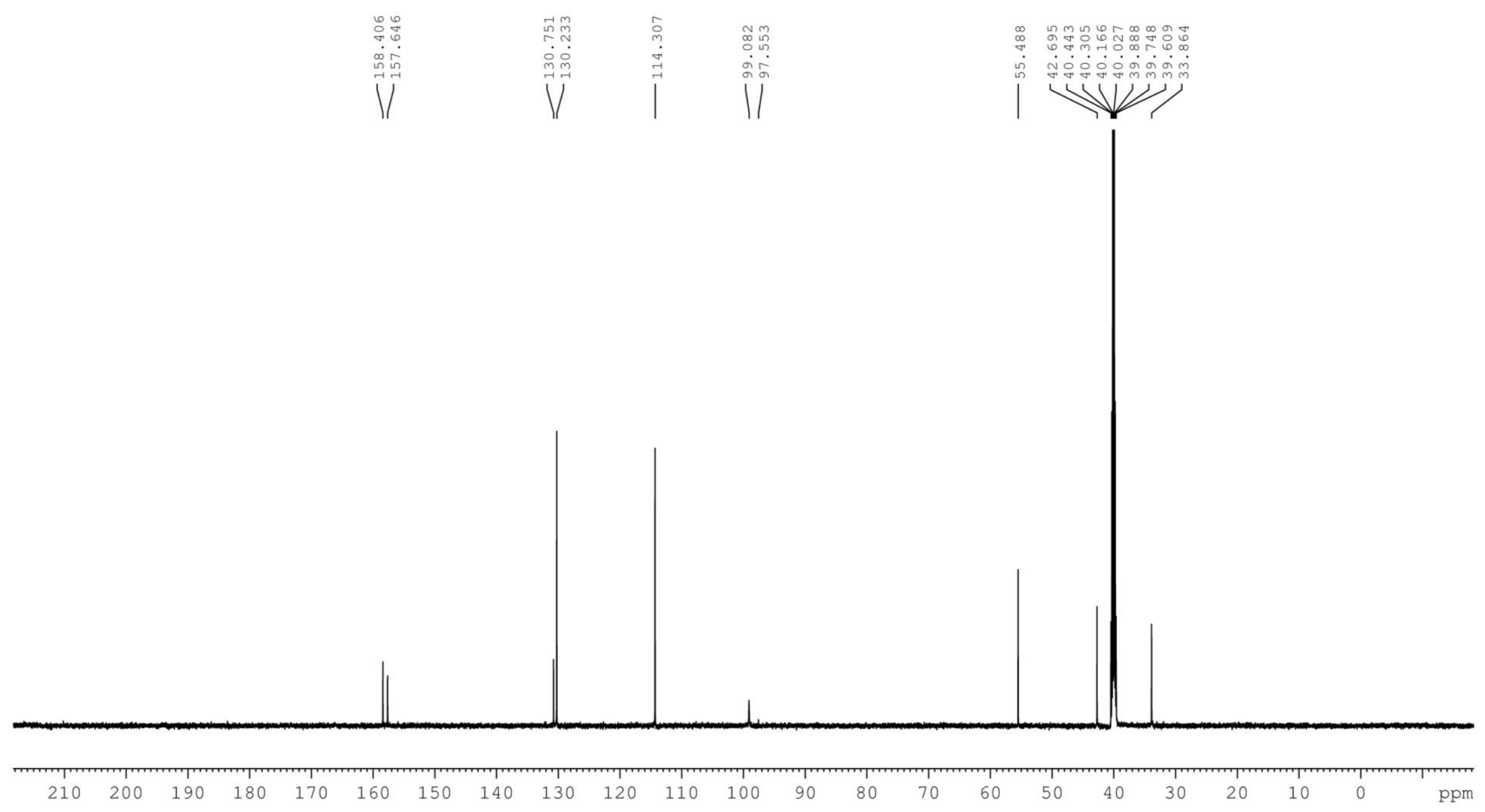

Figure S4. ${ }^{13} \mathrm{C}\left\{{ }^{1} \mathrm{H}\right\}$ NMR (150MHz, DMSO- $\left.d_{6}\right)$ spectra of compound 2a 


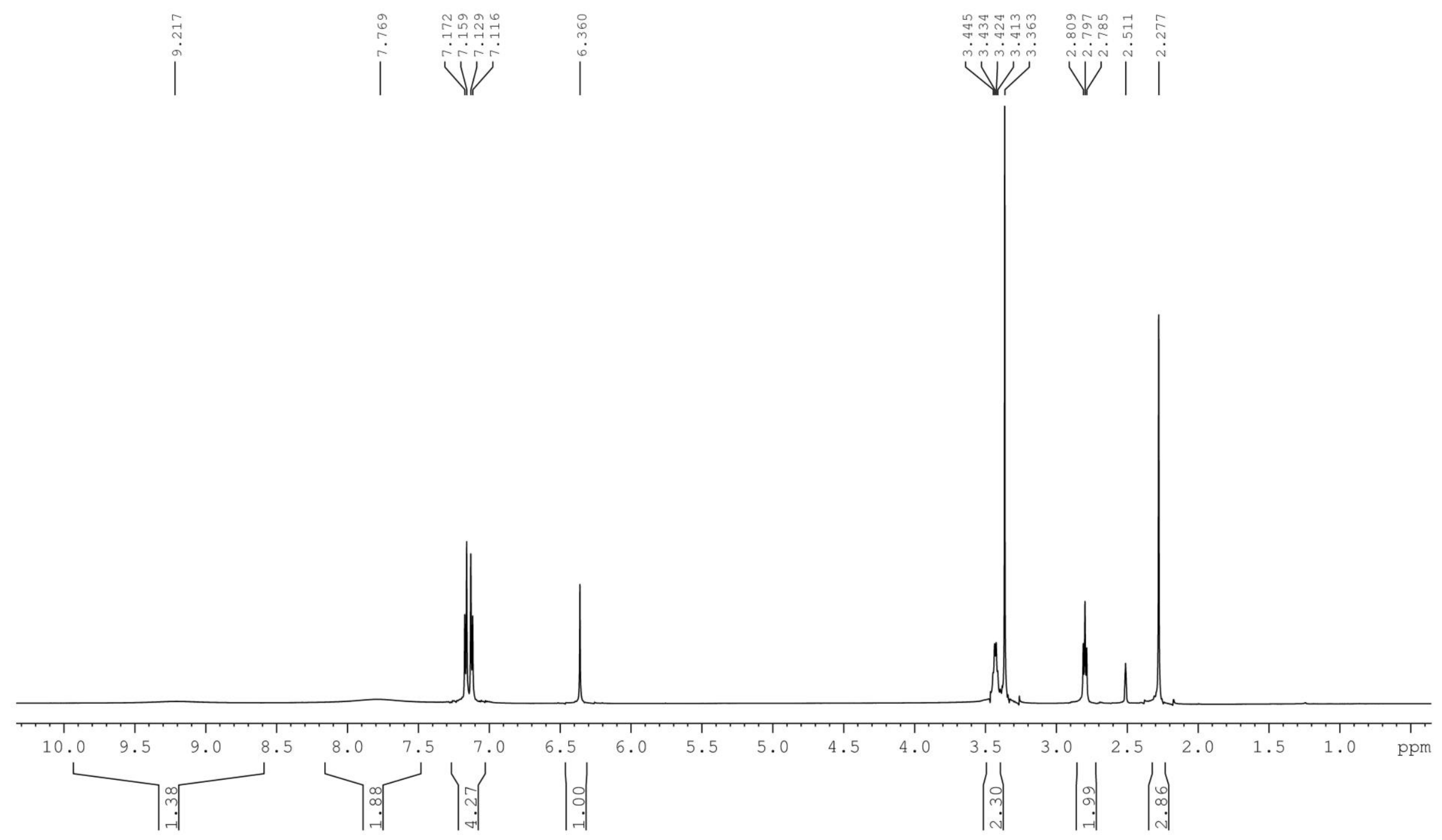

Figure S5. ${ }^{1} \mathrm{H}$ NMR $\left(600 \mathrm{MHz}, \mathrm{DMSO}-d_{6}\right)$ spectra of compound $\mathbf{2 b}$ 


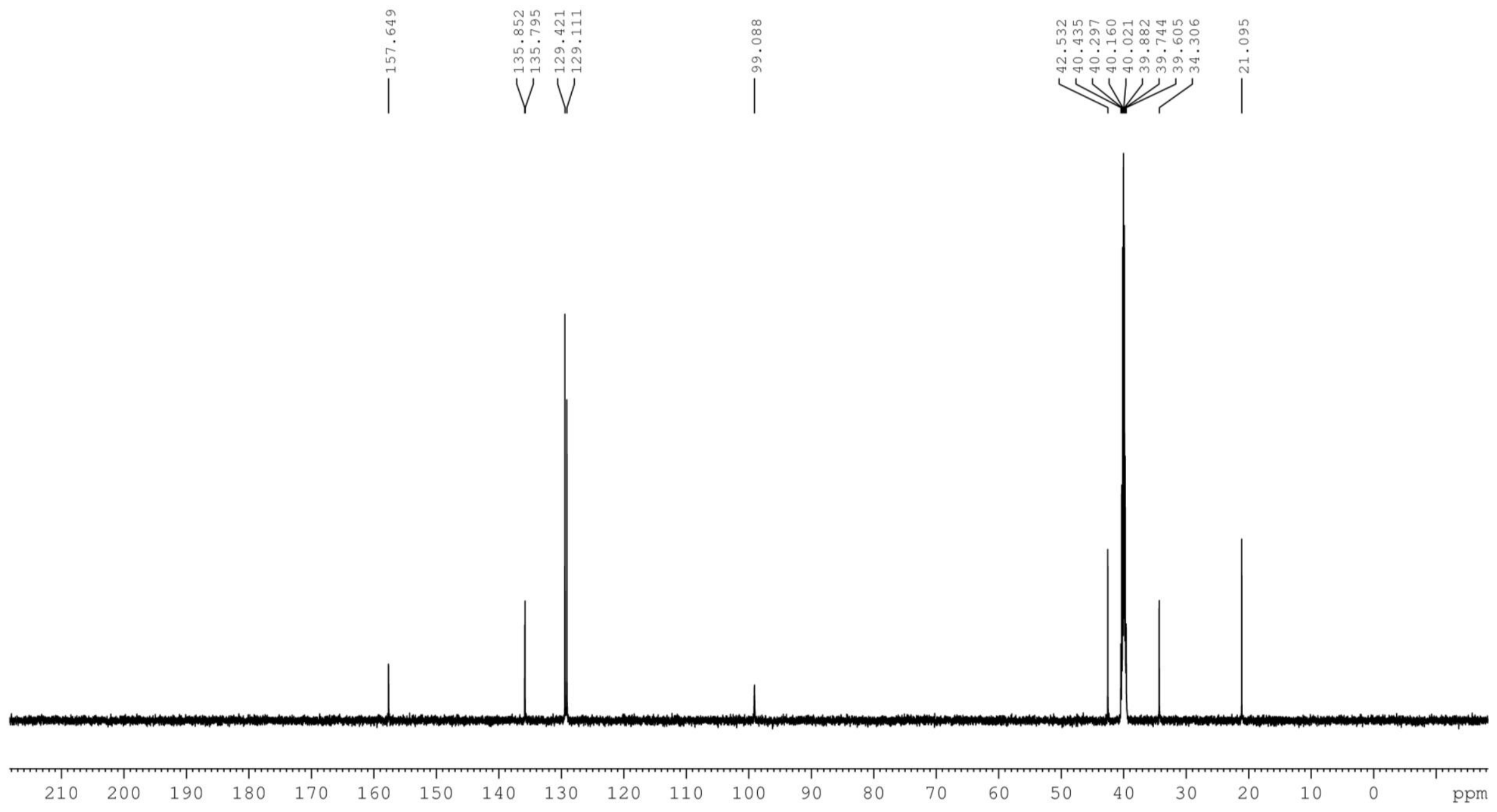

Figure S6. ${ }^{13} \mathrm{C}\left\{{ }^{1} \mathrm{H}\right\}$ NMR $\left(150 \mathrm{MHz}\right.$, DMSO- $\left.d_{6}\right)$ spectra of compound $\mathbf{2 b}$ 


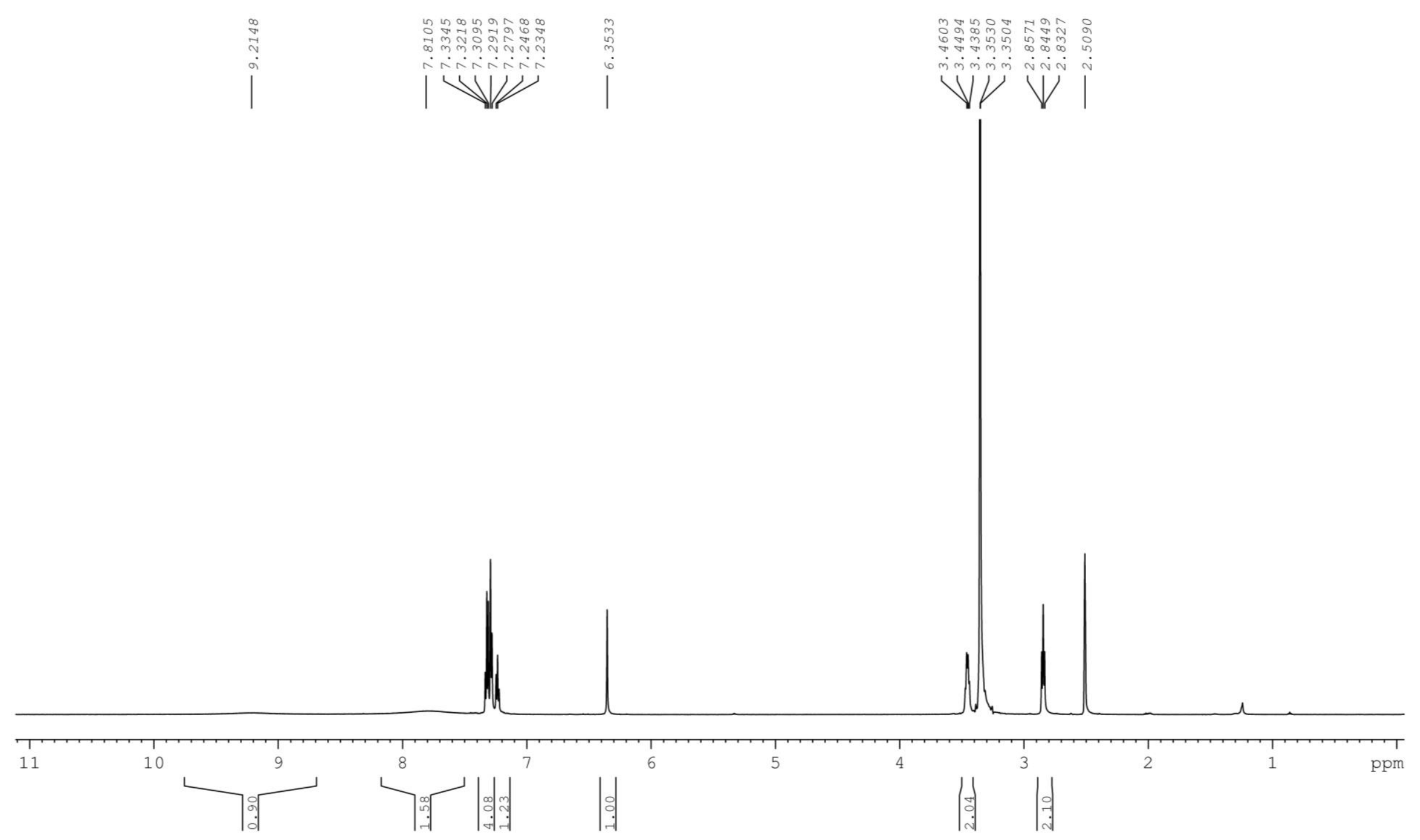

Figure S7. ${ }^{1} \mathrm{H}$ NMR $\left(600 \mathrm{MHz}, \mathrm{DMSO}-d_{6}\right)$ spectra of compound $2 \mathrm{c}$ 


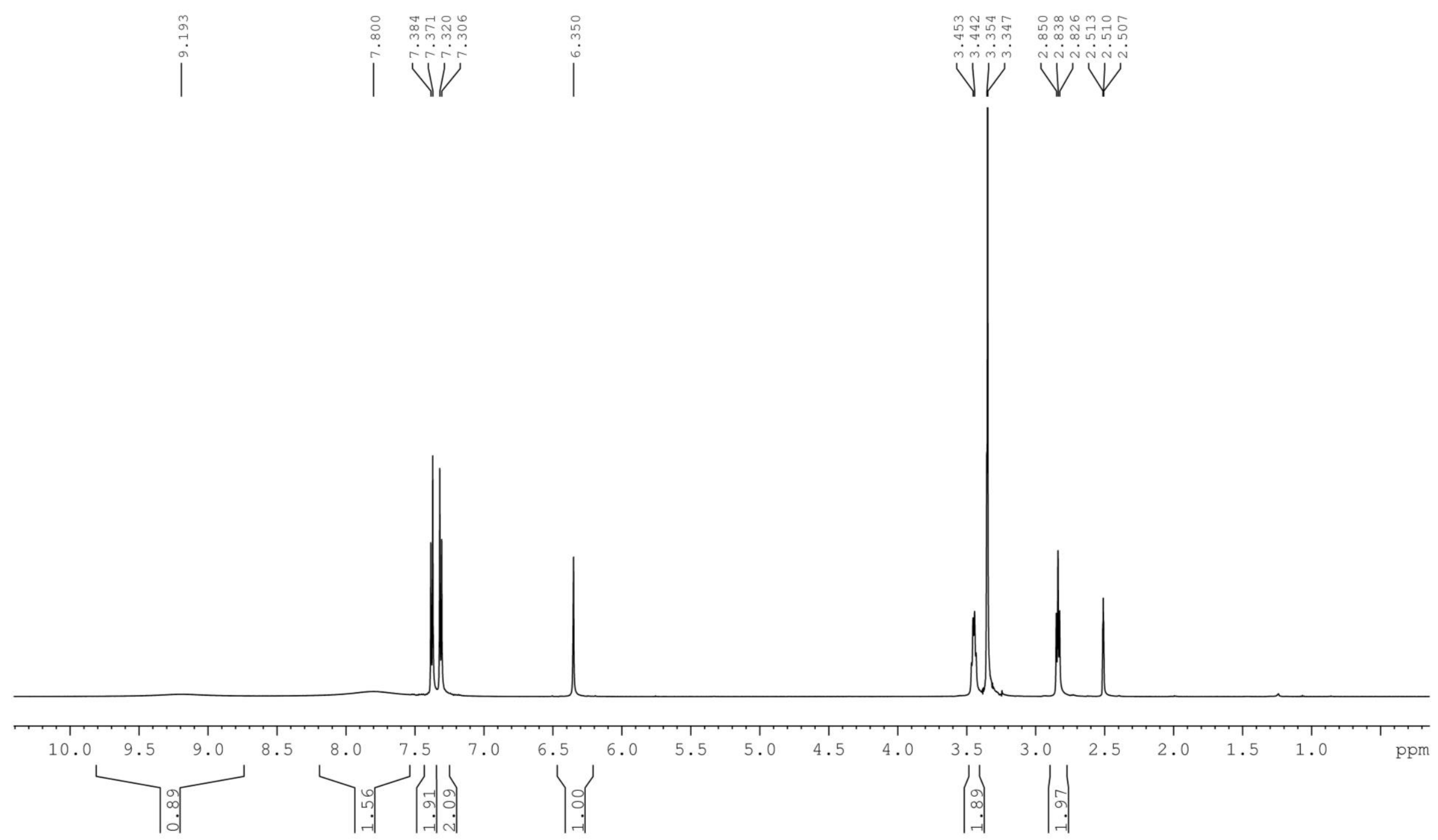

Figure S8. ${ }^{1} \mathrm{H}$ NMR $\left(600 \mathrm{MHz}, \mathrm{DMSO}-d_{6}\right)$ spectra of compound 2d 


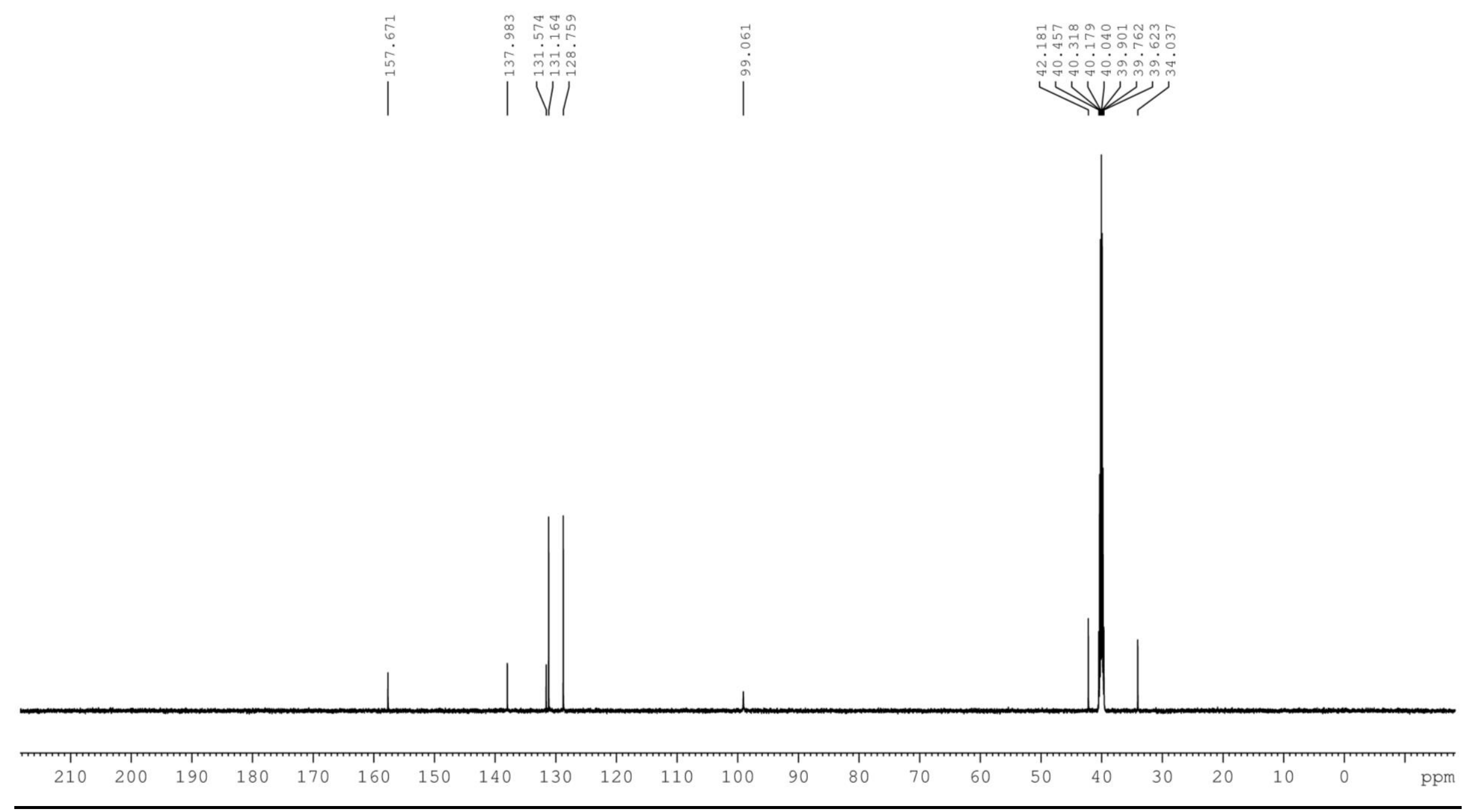

Figure S9. ${ }^{13} \mathrm{C}\left\{{ }^{1} \mathrm{H}\right\}$ NMR $\left(150 \mathrm{MHz}\right.$, DMSO- $\left.d_{6}\right)$ spectra of compound 2d 


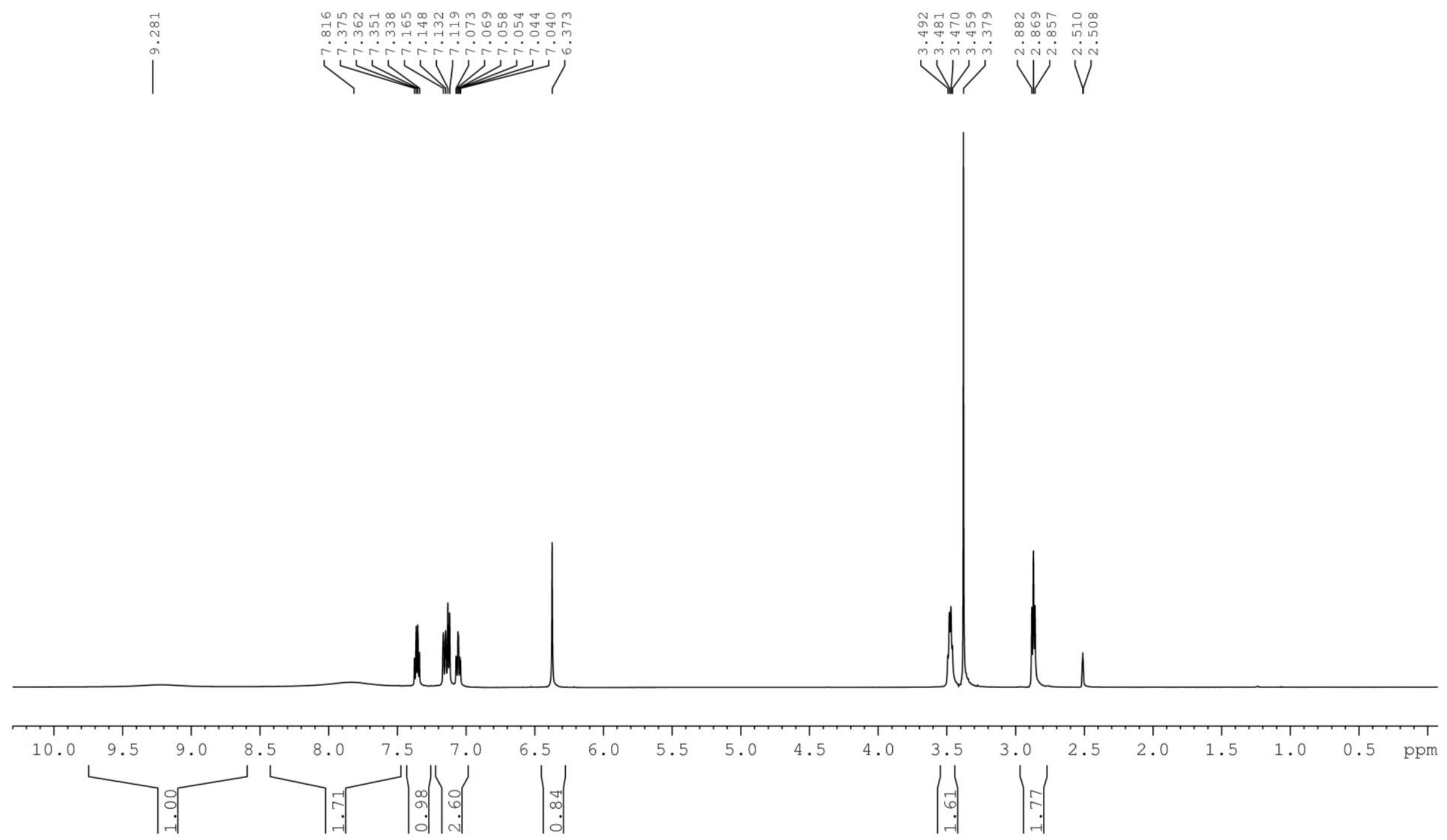

Figure S10. ${ }^{1} \mathrm{H}$ NMR $\left(600 \mathrm{MHz}, \mathrm{DMSO}-d_{6}\right)$ spectra of compound $2 \mathbf{e}$ 

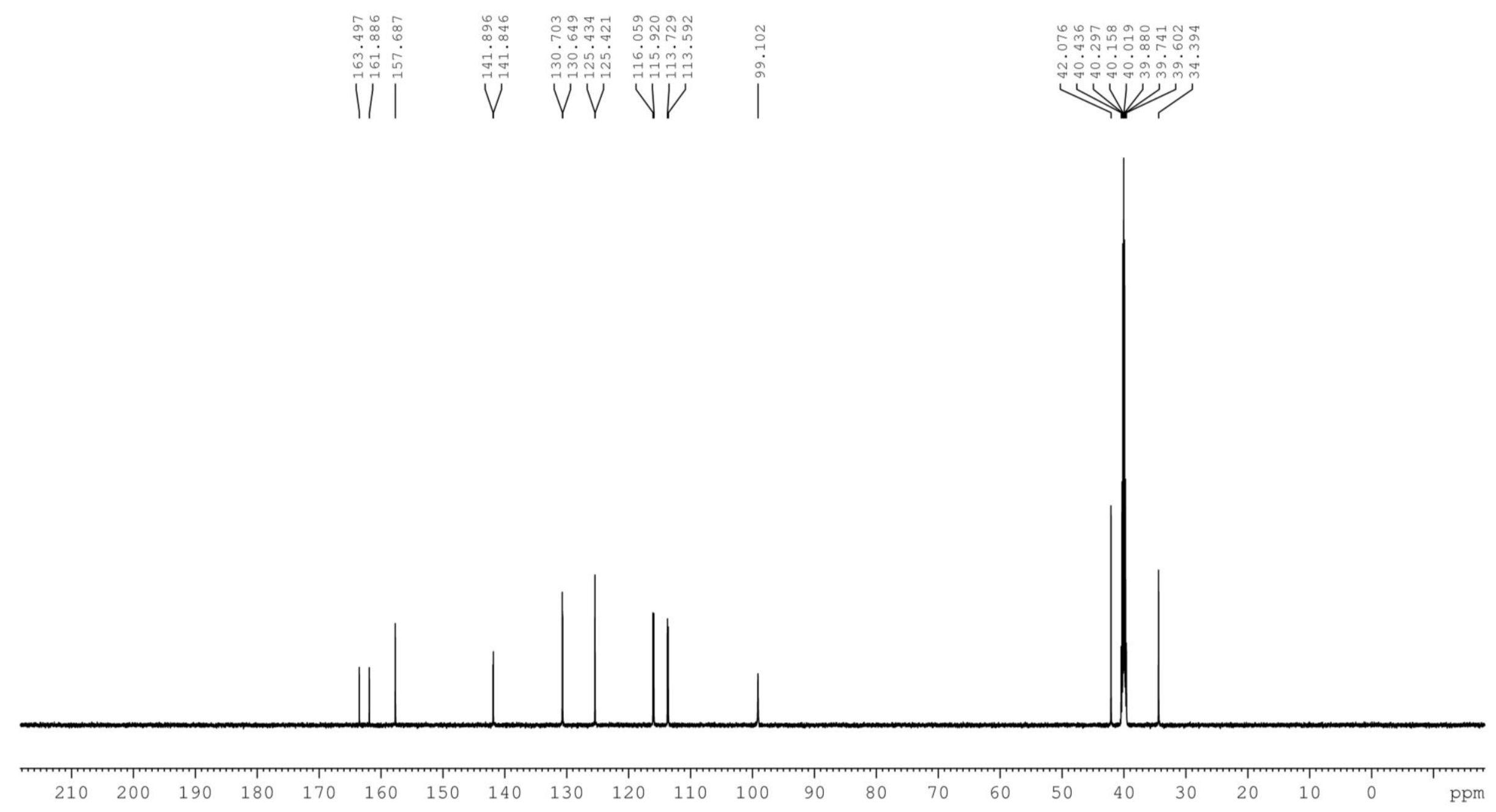

Figure S11. ${ }^{13} \mathrm{C}\left\{{ }^{1} \mathrm{H}\right\}$ NMR $\left(150 \mathrm{MHz}, \mathrm{DMSO}-d_{6}\right)$ spectra of compound $2 \mathbf{e}$ 


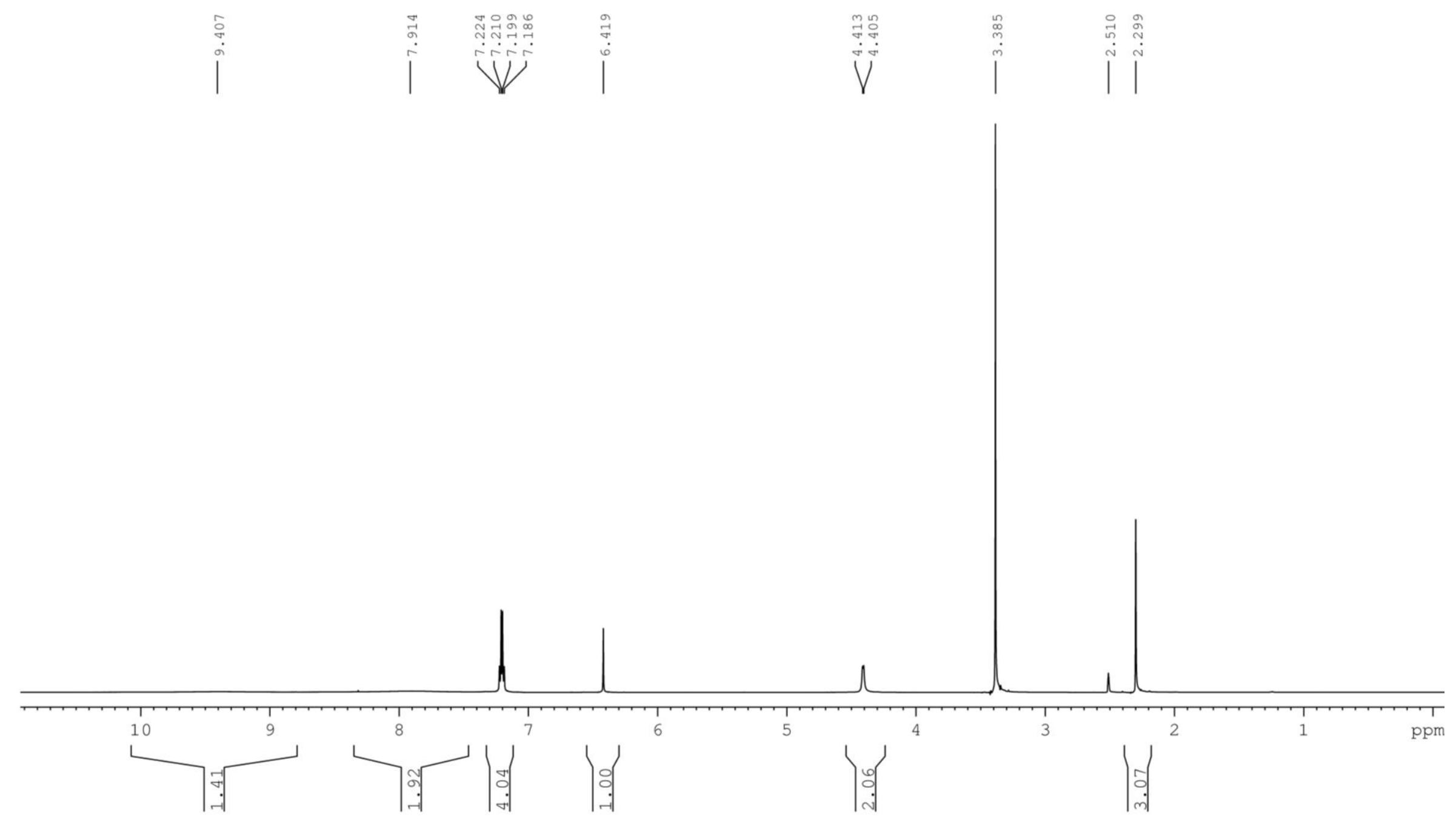

Figure S12. ${ }^{1} \mathrm{H}$ NMR $\left(600 \mathrm{MHz}, \mathrm{DMSO}-d_{6}\right)$ spectra of compound $\mathbf{2 f}$ 


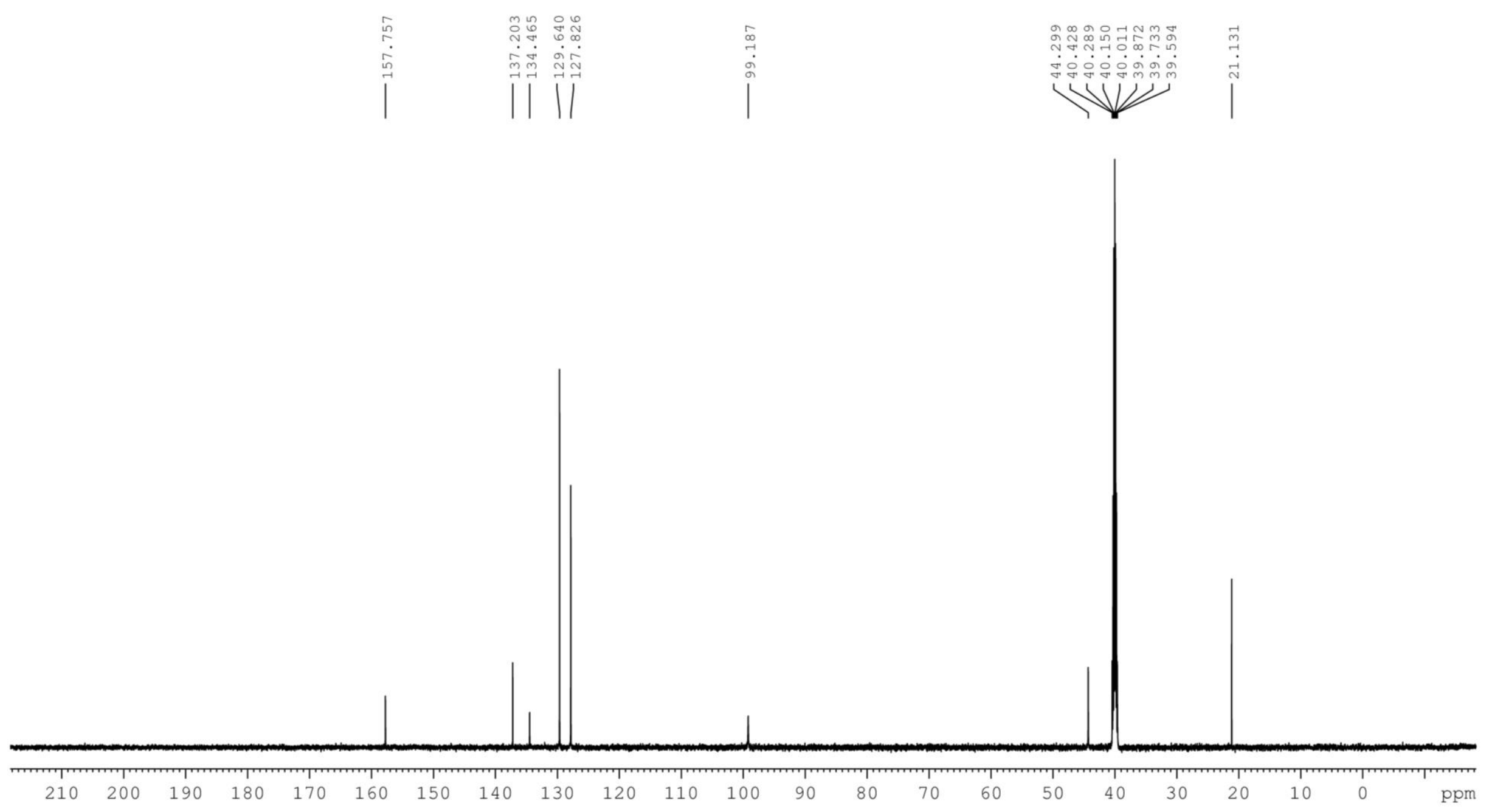

Figure S13. ${ }^{13} \mathrm{C}\left\{{ }^{1} \mathrm{H}\right\}$ NMR (150MHz, DMSO- $\left.d_{6}\right)$ spectra of compound $2 \mathbf{f}$ 


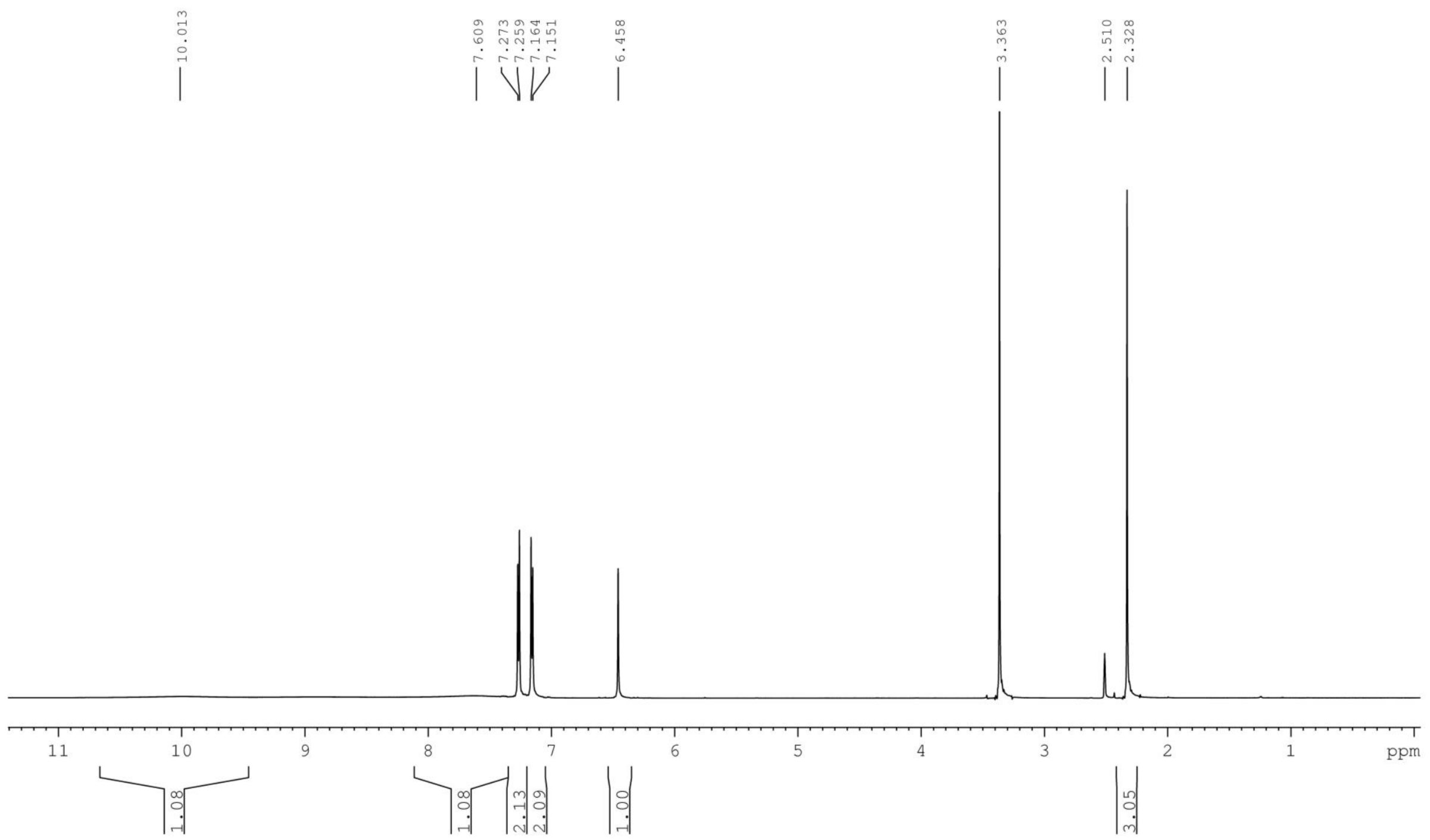

Figure S14. ${ }^{1} \mathrm{H}$ NMR (600 MHz, DMSO- $\left.d_{6}\right)$ spectra of compound $2 \mathrm{~g}$

$\mathrm{S} 24$ 


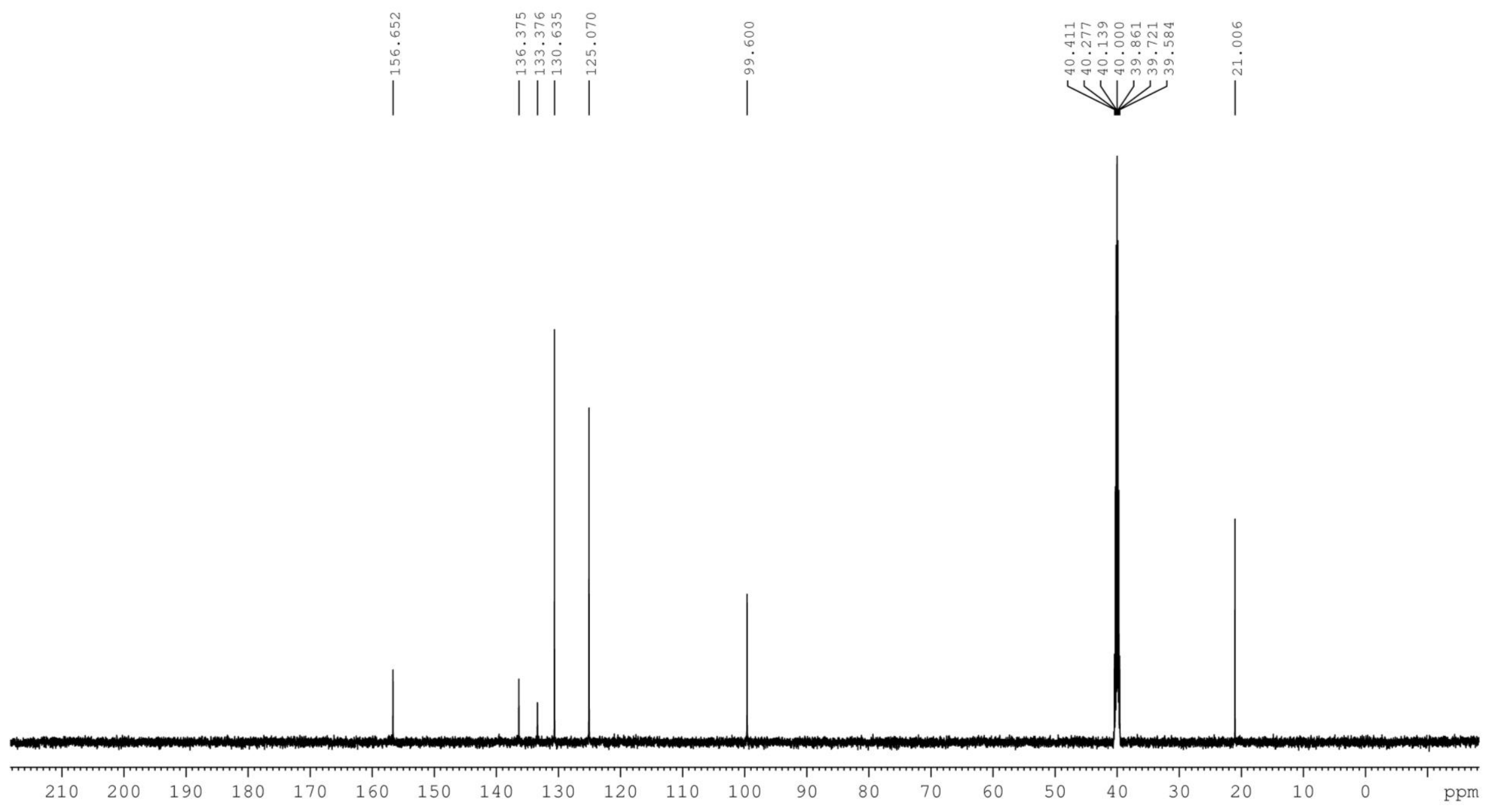

Figure S15. ${ }^{13} \mathrm{C}\left\{{ }^{1} \mathrm{H}\right\}$ NMR $\left(150 \mathrm{MHz}\right.$, DMSO- $\left.d_{6}\right)$ spectra of compound $\mathbf{2 g}$ 


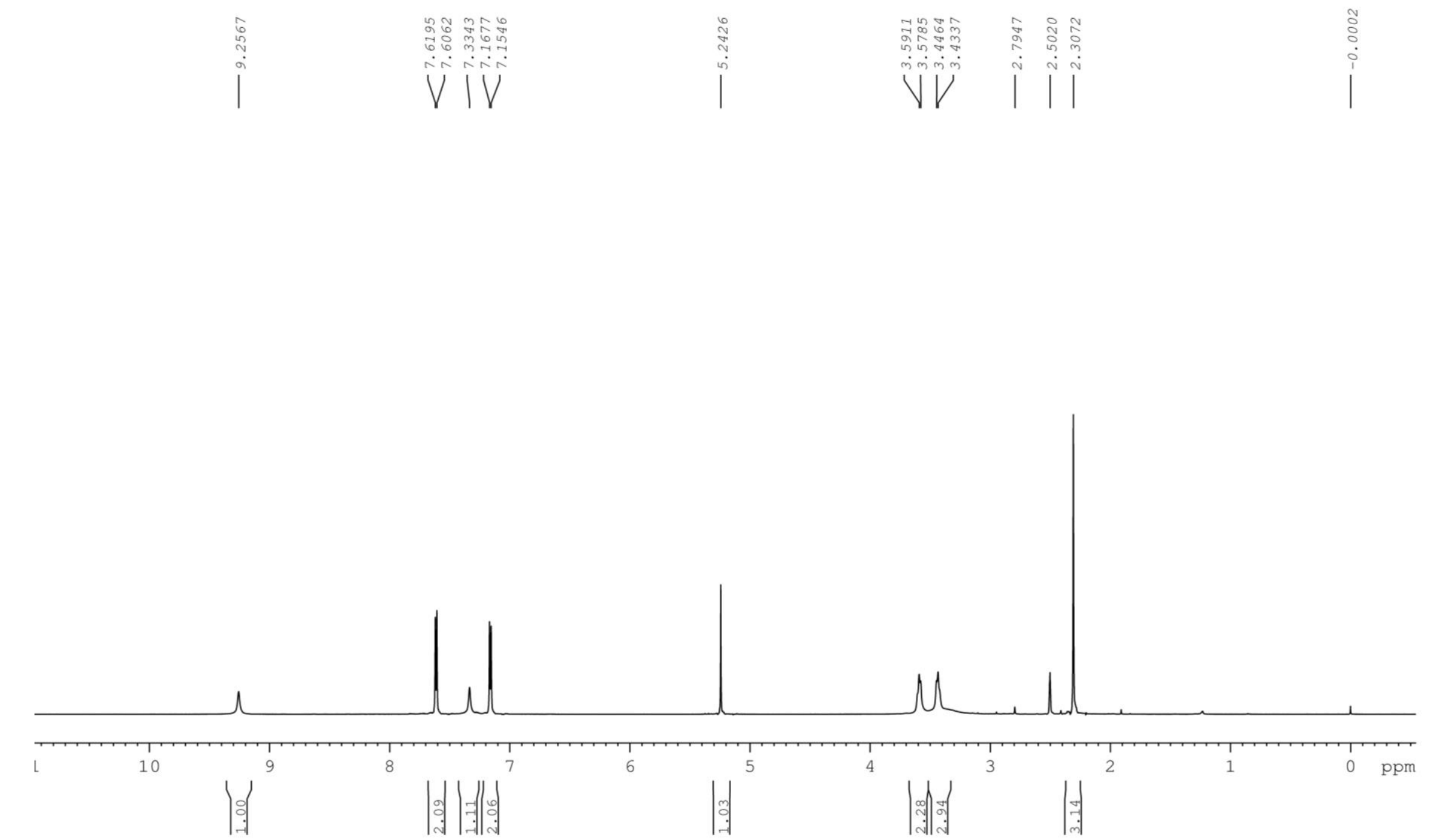

Figure S16. ${ }^{1} \mathrm{H}$ NMR $\left(600 \mathrm{MHz}, \mathrm{DMSO}-d_{6}\right)$ spectra of compound 3a 


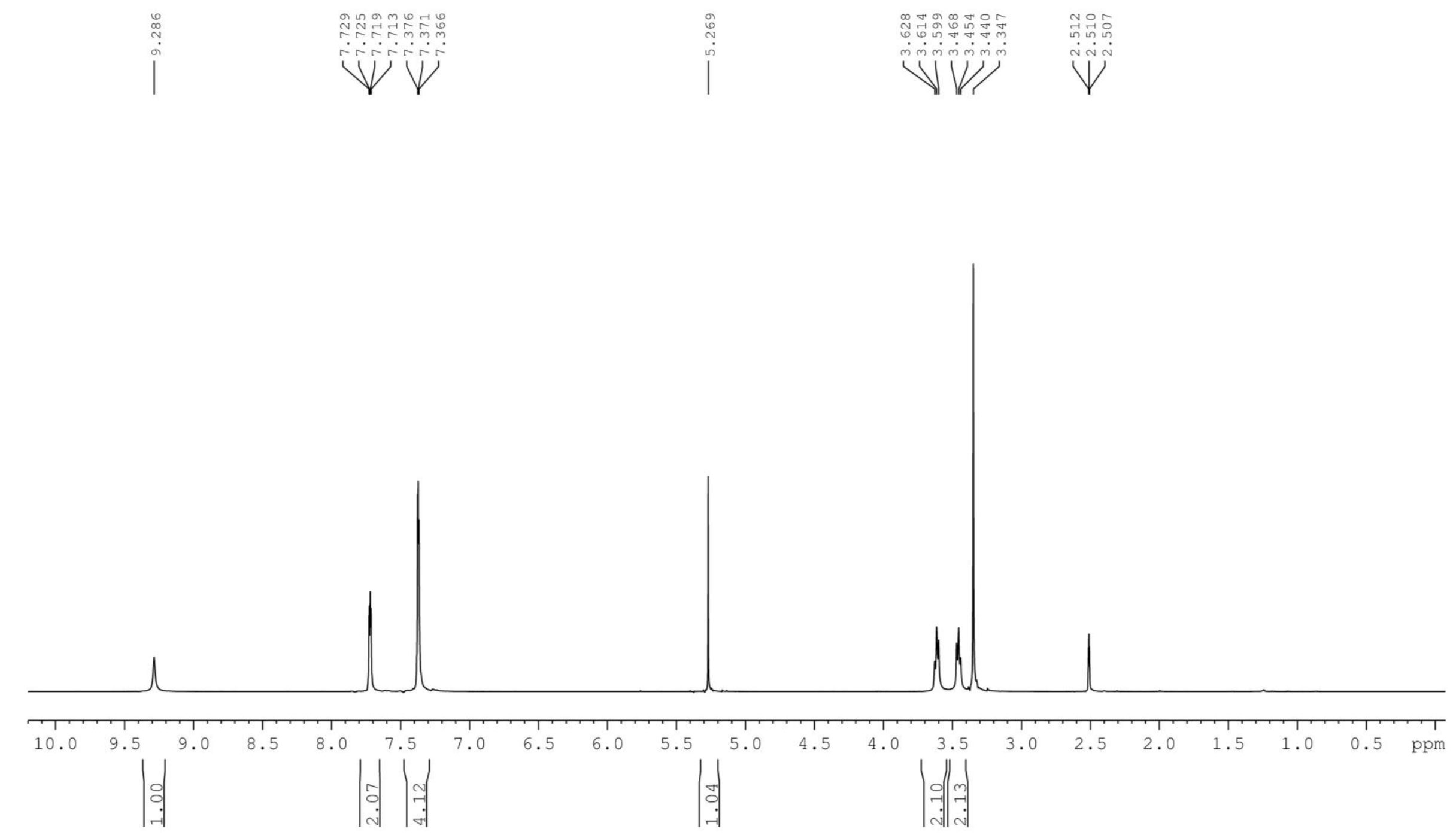

Figure S17. ${ }^{1} \mathrm{H}$ NMR $\left(600 \mathrm{MHz}\right.$, DMSO- $\left.d_{6}\right)$ spectra of compound $\mathbf{3 b}$ 


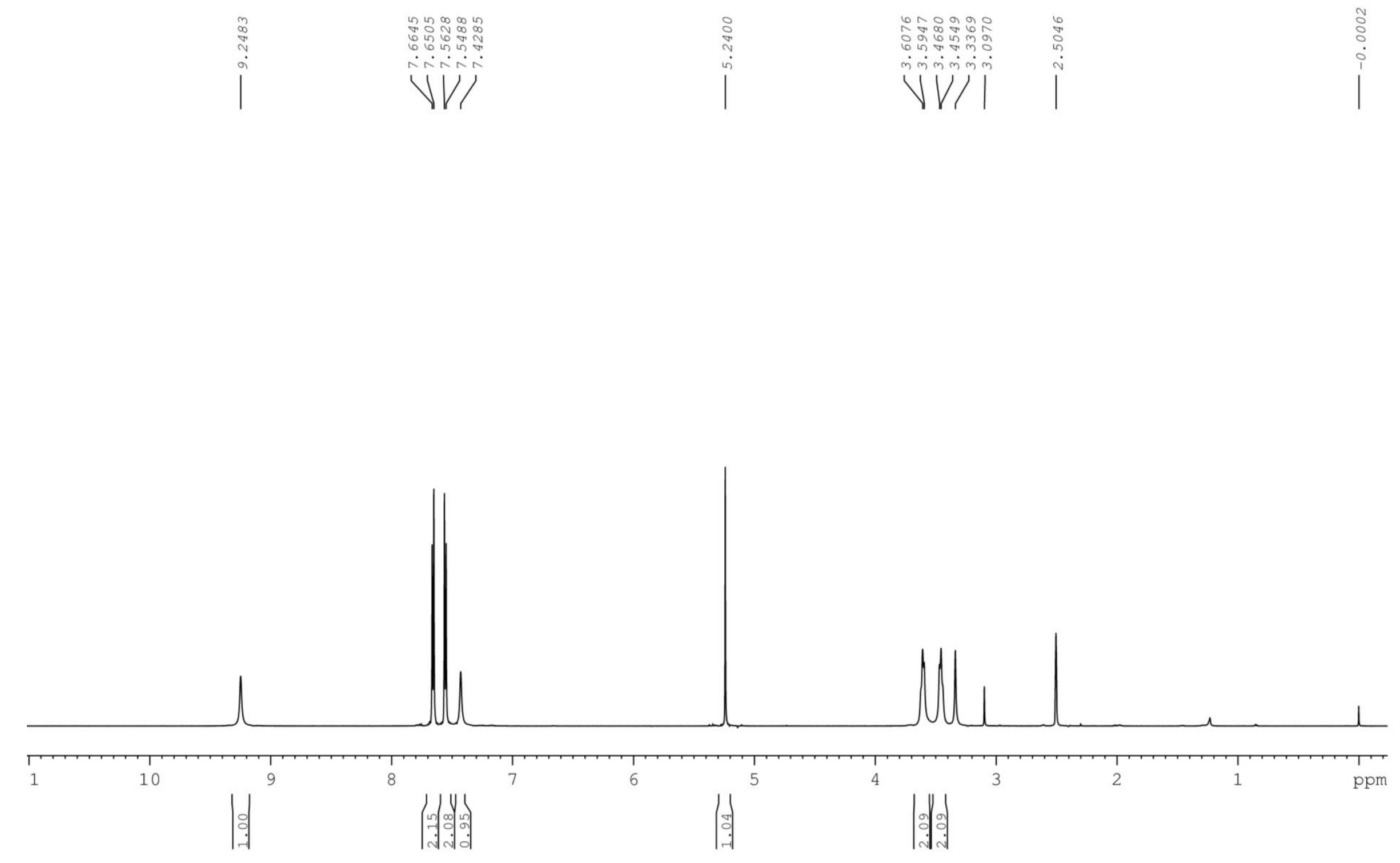

Figure S18. ${ }^{1} \mathrm{H}$ NMR (600 MHz, DMSO- $\left.d_{6}\right)$ spectra of compound 3c 


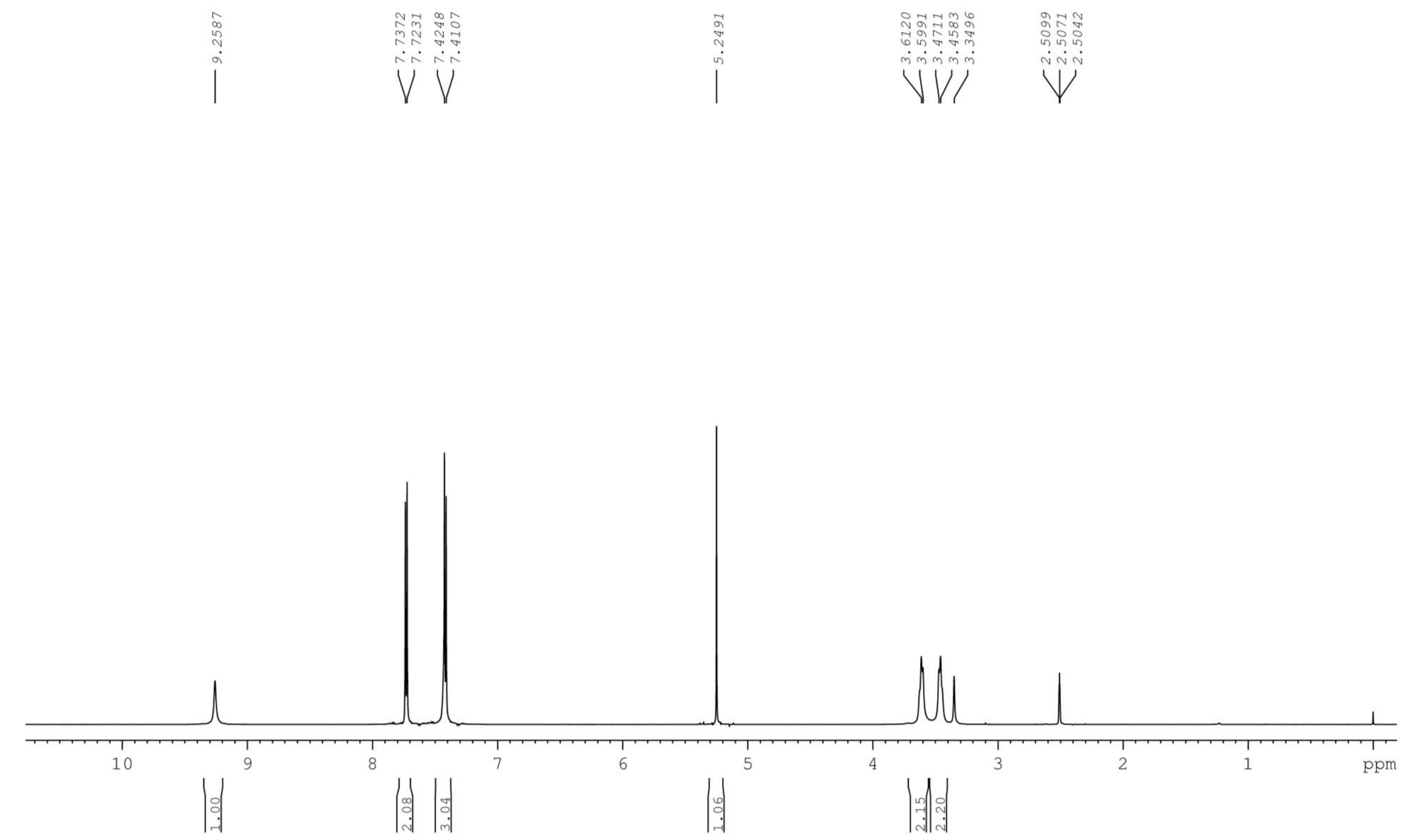

Figure S19. ${ }^{1} \mathrm{H}$ NMR (600 MHz, DMSO- $\left.d_{6}\right)$ spectra of compound 3d 


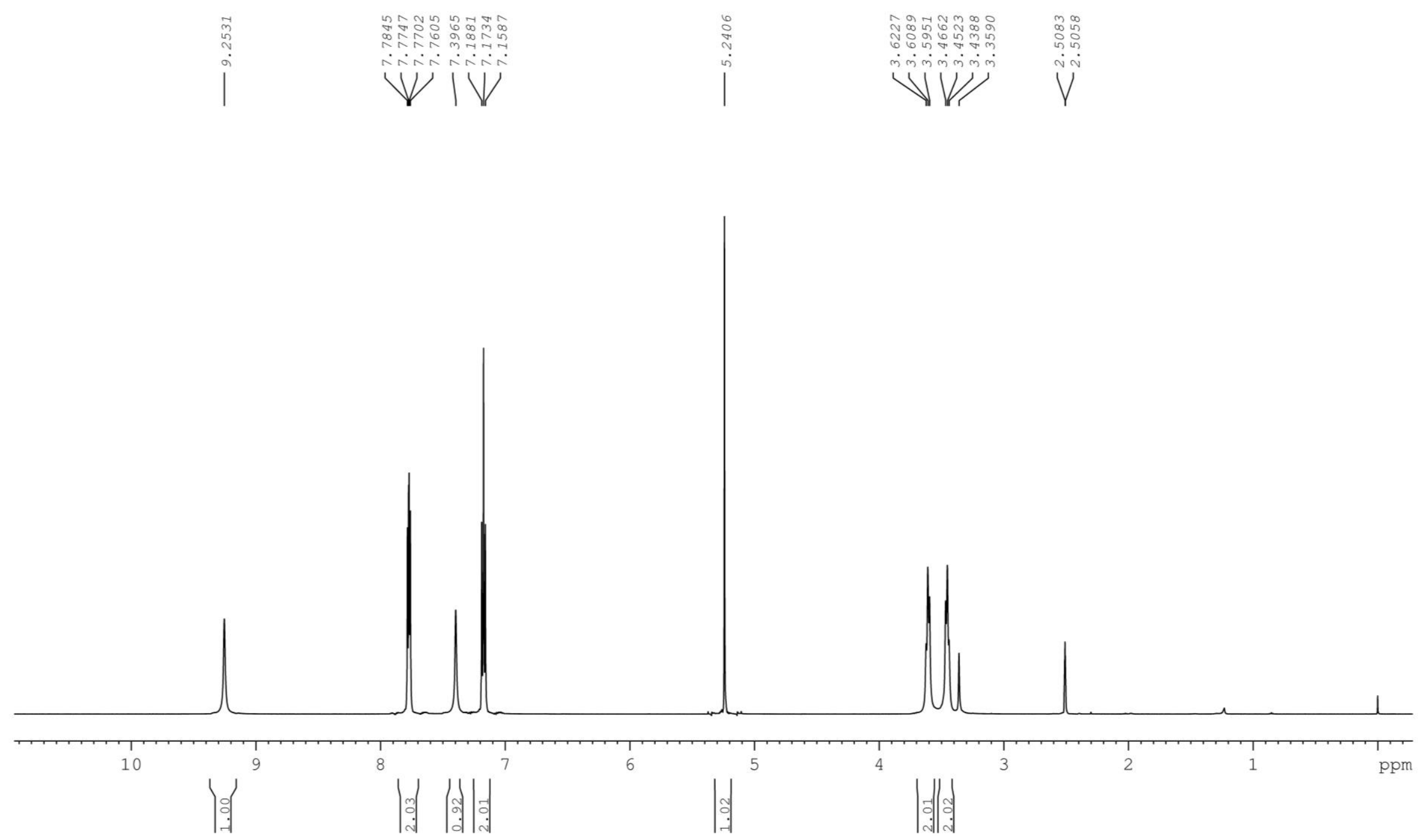

Figure S20. ${ }^{1} \mathrm{H}$ NMR $\left(600 \mathrm{MHz}, \mathrm{DMSO}-d_{6}\right)$ spectra of compound 3e 


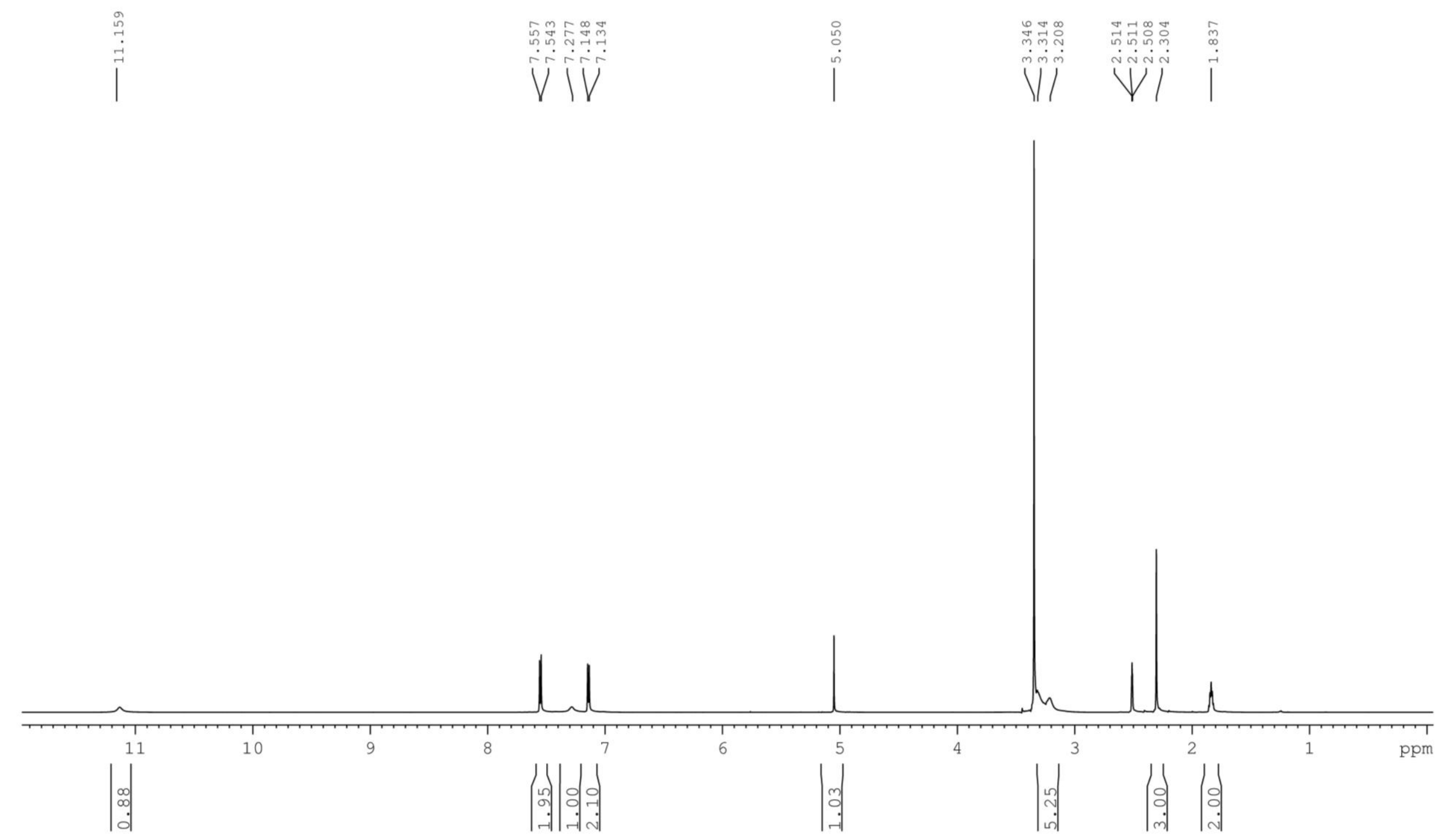

Figure S21. ${ }^{1} \mathrm{H}$ NMR (600 MHz, DMSO- $d_{6}$ ) spectra of compound $3 \mathbf{f}$ 


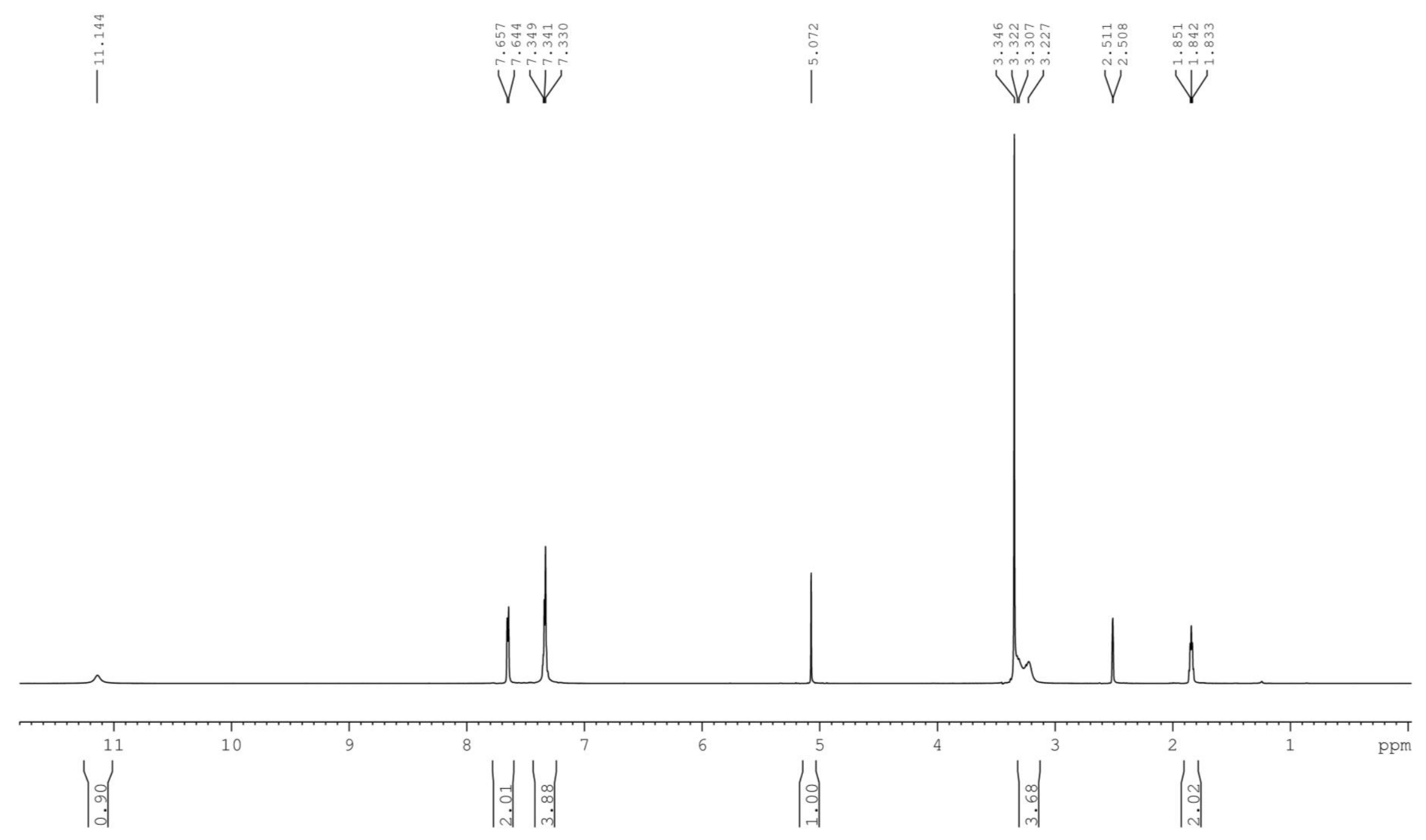

Figure S22. ${ }^{1} \mathrm{H}$ NMR (600 MHz, DMSO- $\left.d_{6}\right)$ spectra of compound 3g 

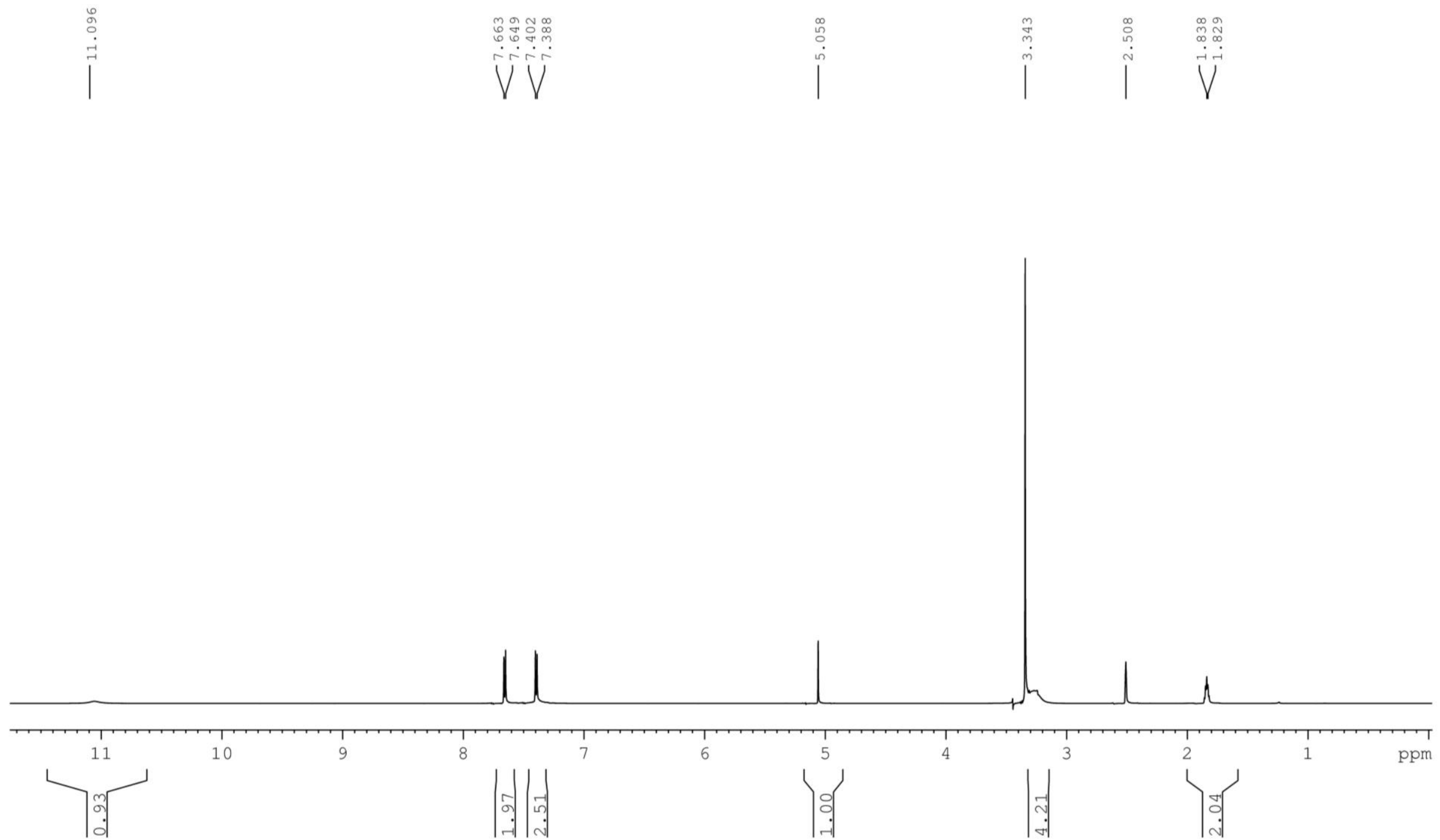

Figure S23. ${ }^{1} \mathrm{H}$ NMR $\left(600 \mathrm{MHz}\right.$, DMSO- $\left.d_{6}\right)$ spectra of compound $\mathbf{3 h}$ 


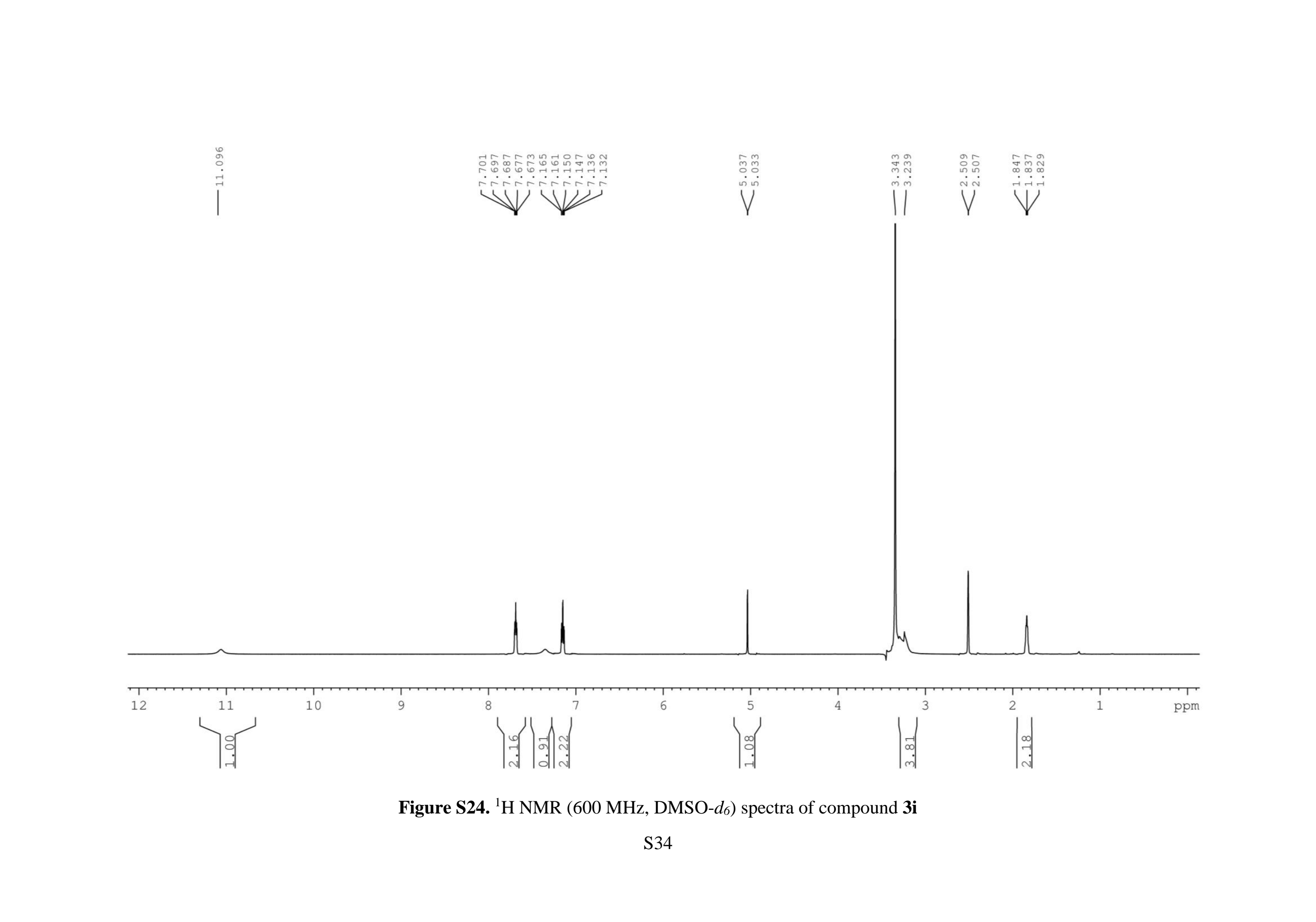



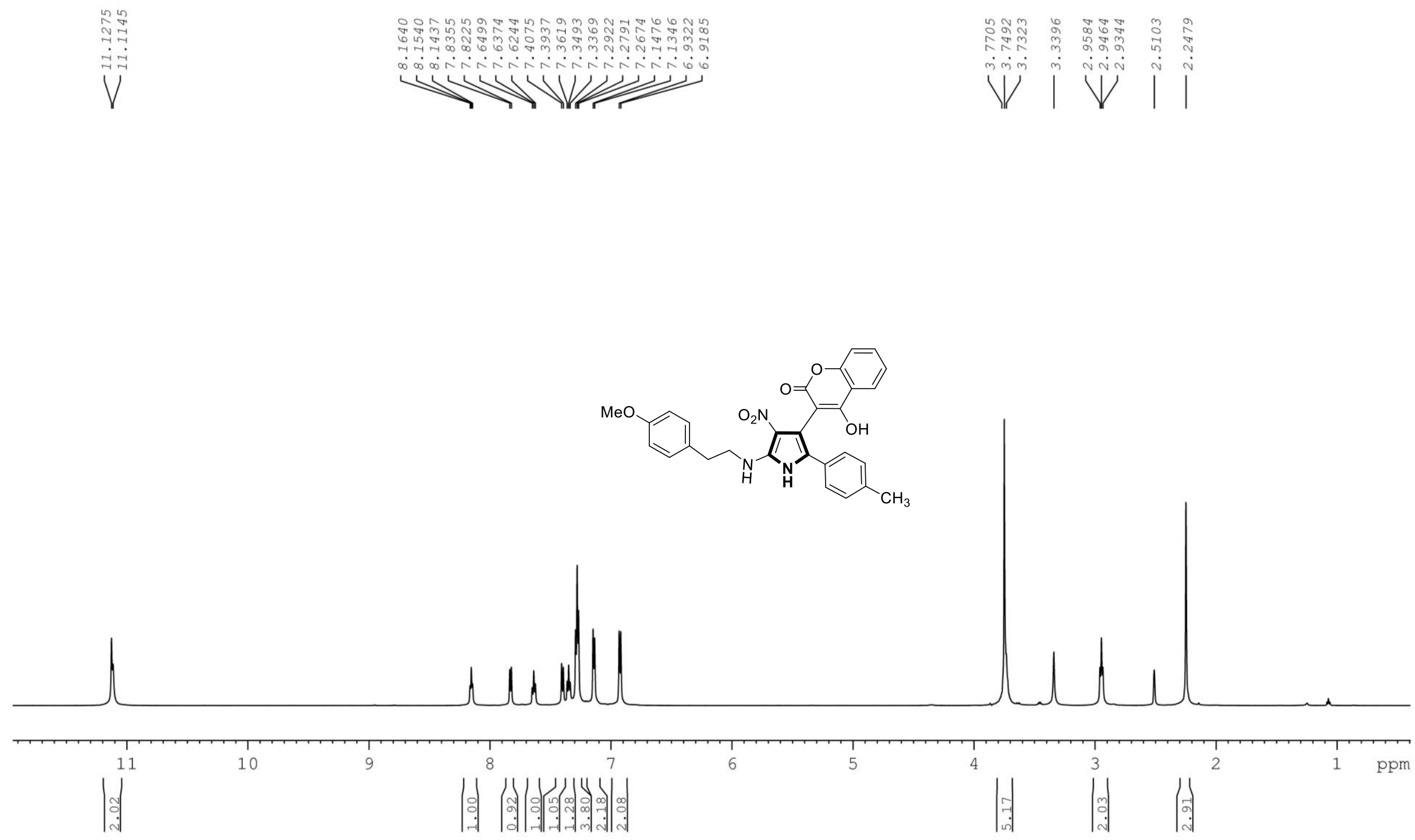

Figure S25. ${ }^{1} \mathrm{H}$ NMR (600 MHz, DMSO- $\left.d_{6}\right)$ spectra of compound 5a 


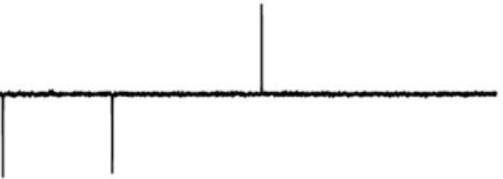

YUNNAN UNIVERSITY ASCEND AVIIIHD600 ZQX-a-47 Jan25-2019-ziquanxing C13CPD DMSO
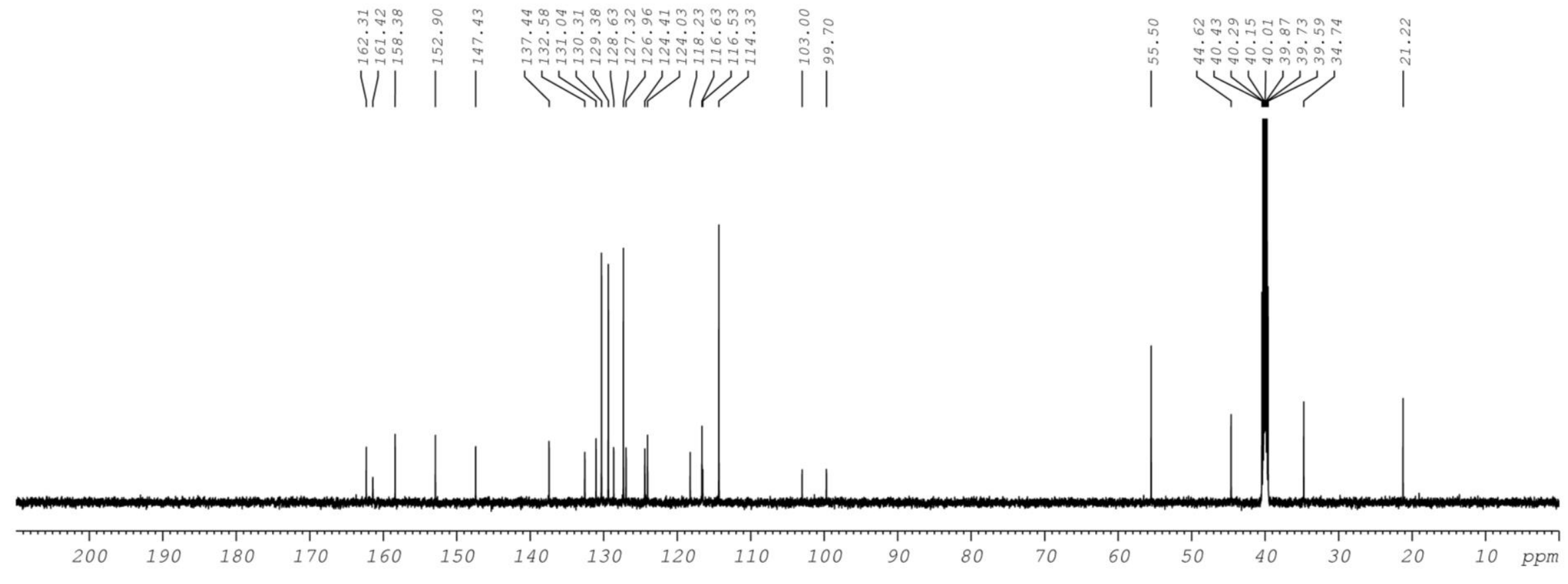

Figure S26. ${ }^{13} \mathrm{C}\left\{{ }^{1} \mathrm{H}\right\}$ NMR $\left(150 \mathrm{MHz}\right.$, DMSO- $\left.d_{6}\right)$ spectra of compound 5a 


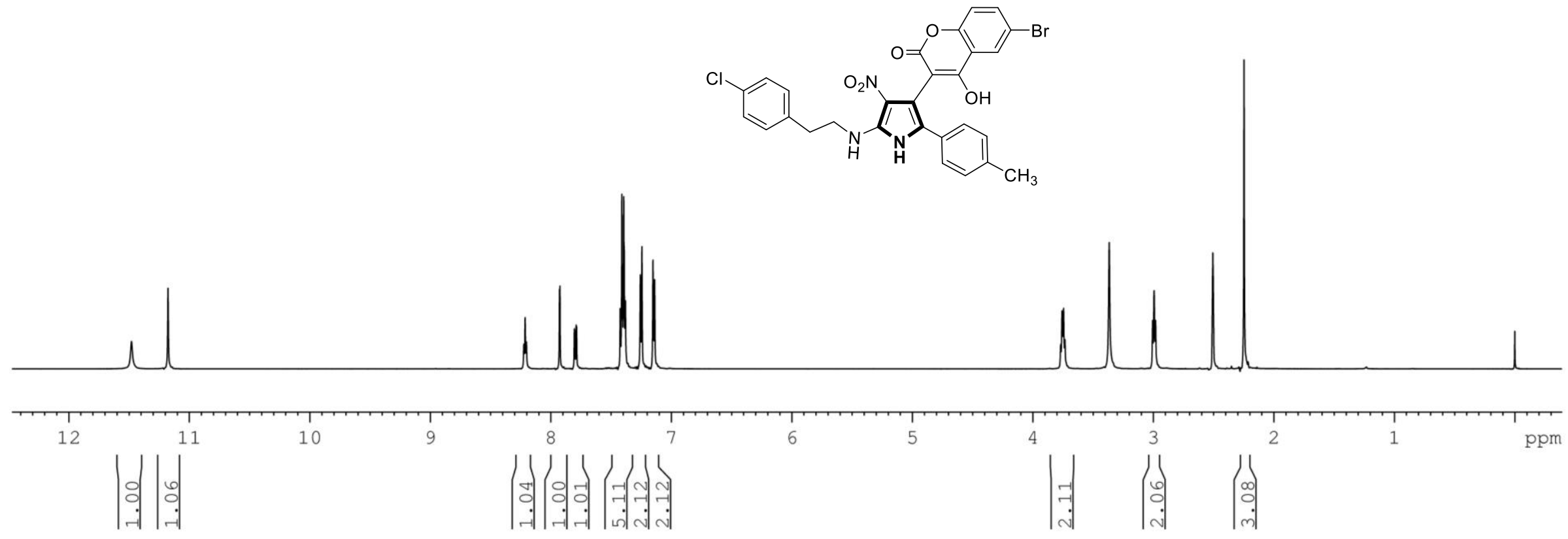

Figure S27. ${ }^{1} \mathrm{H}$ NMR (600 MHz, DMSO- $d_{6}$ ) spectra of compound $\mathbf{5 b}$ 


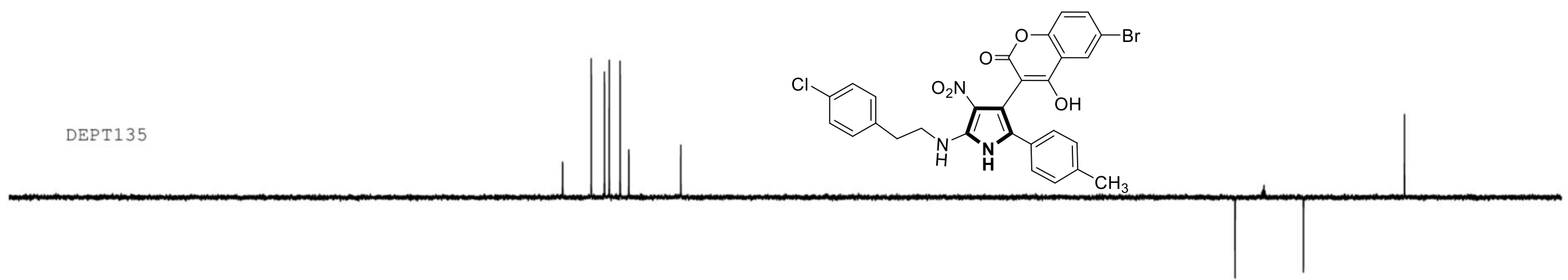

YUNNAN UNIVERSITY ASCEND AVIIIHD600 ZQX-a-48 Jan26-2019-ziquanxing C13CPD DMSO
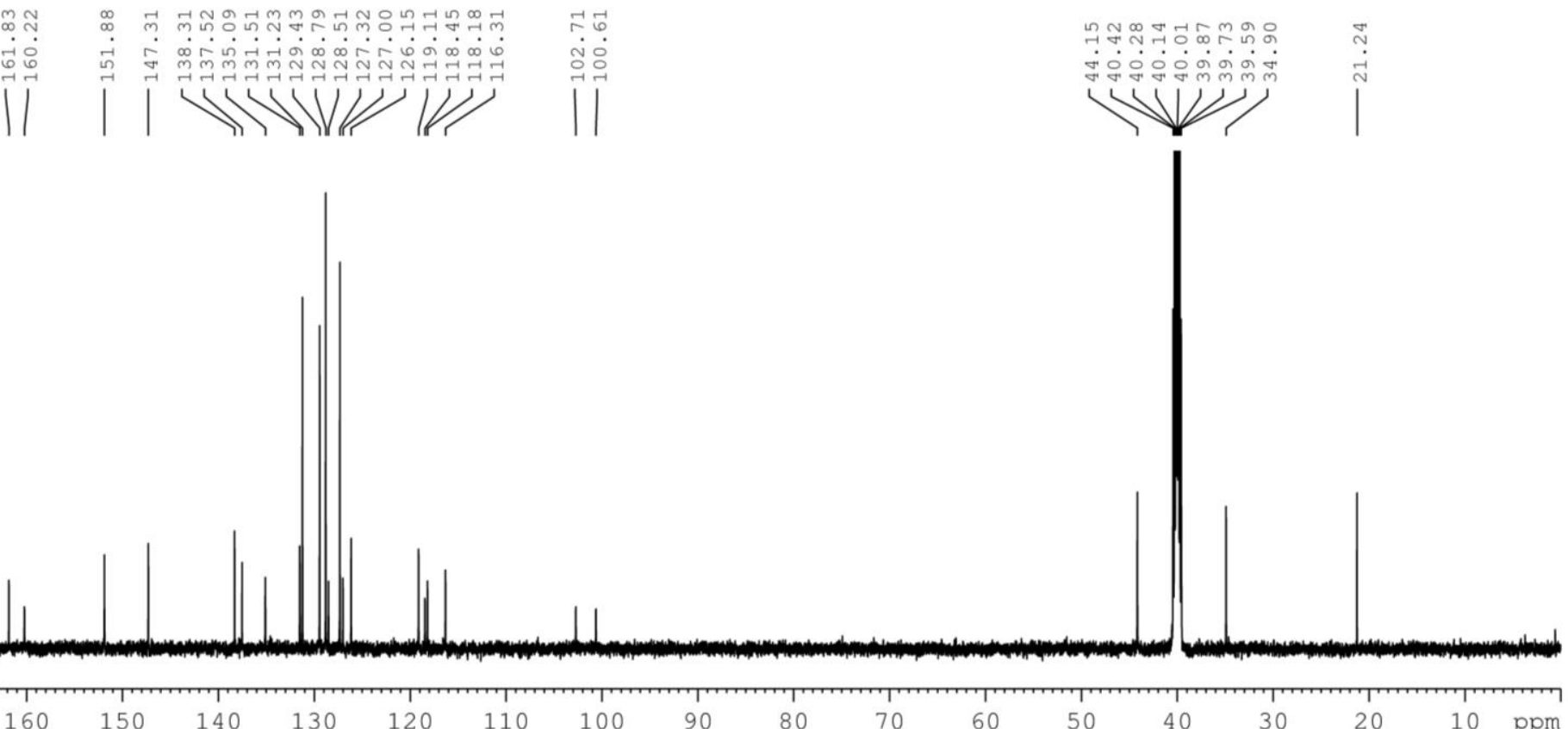

Figure S28. ${ }^{13} \mathrm{C}\left\{{ }^{1} \mathrm{H}\right\}$ NMR $\left(150 \mathrm{MHz}\right.$, DMSO- $\left.d_{6}\right)$ spectra of compound $\mathbf{5 b}$ 

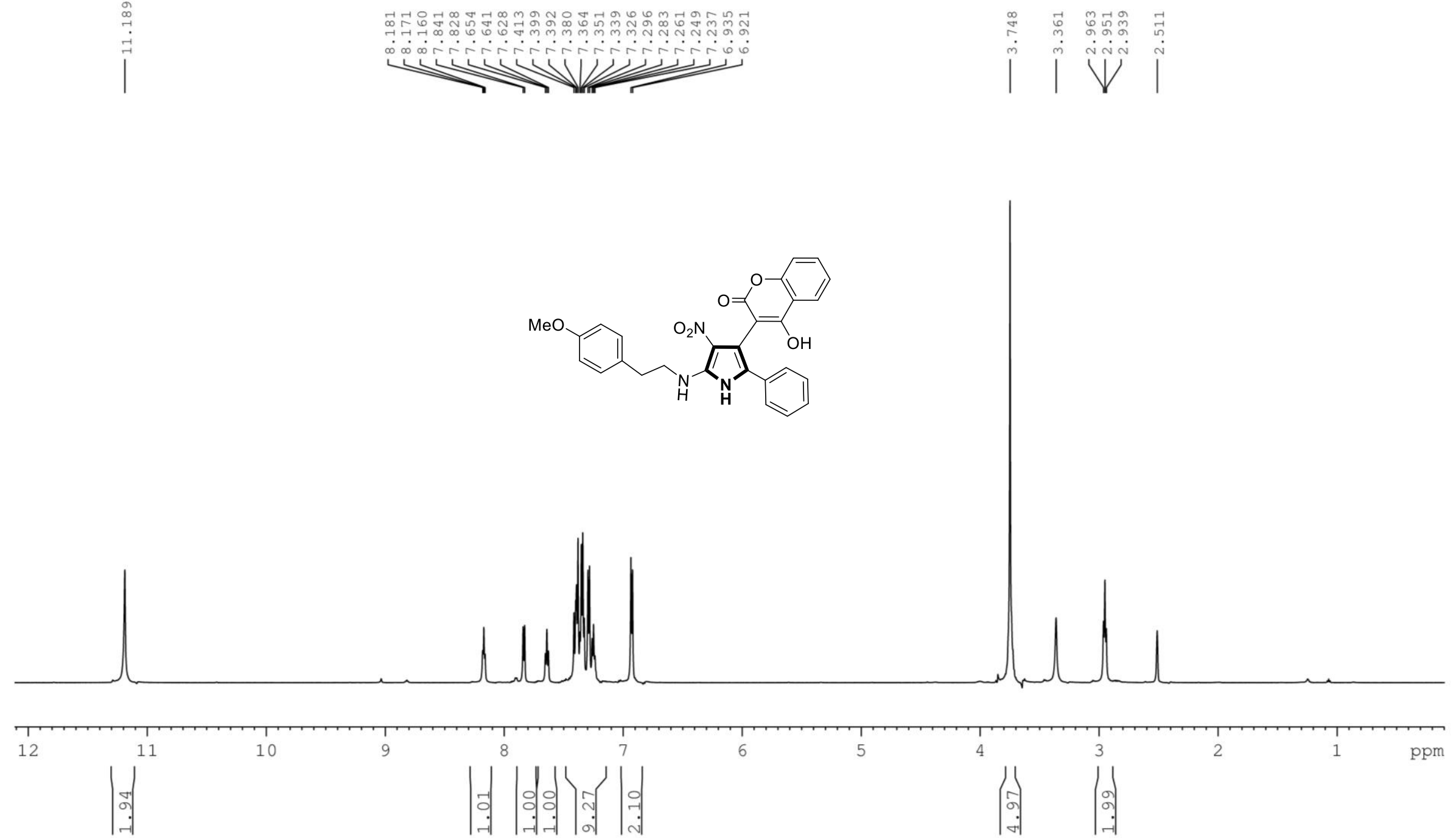

Figure S29. ${ }^{1} \mathrm{H}$ NMR $\left(600 \mathrm{MHz}, \mathrm{DMSO}-d_{6}\right)$ spectra of compound 5c 


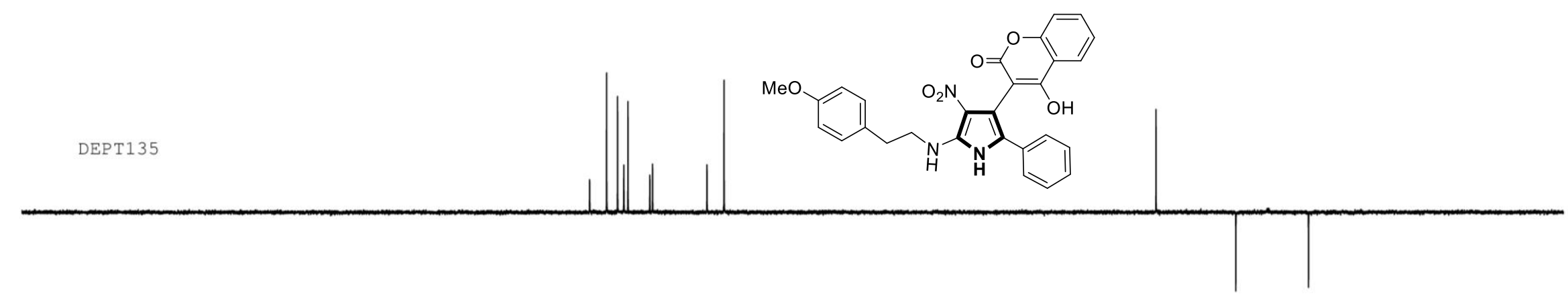

YUNNAN UNIVERSITY ASCEND AVIIIHD600 A-42 C13CPD DMSO

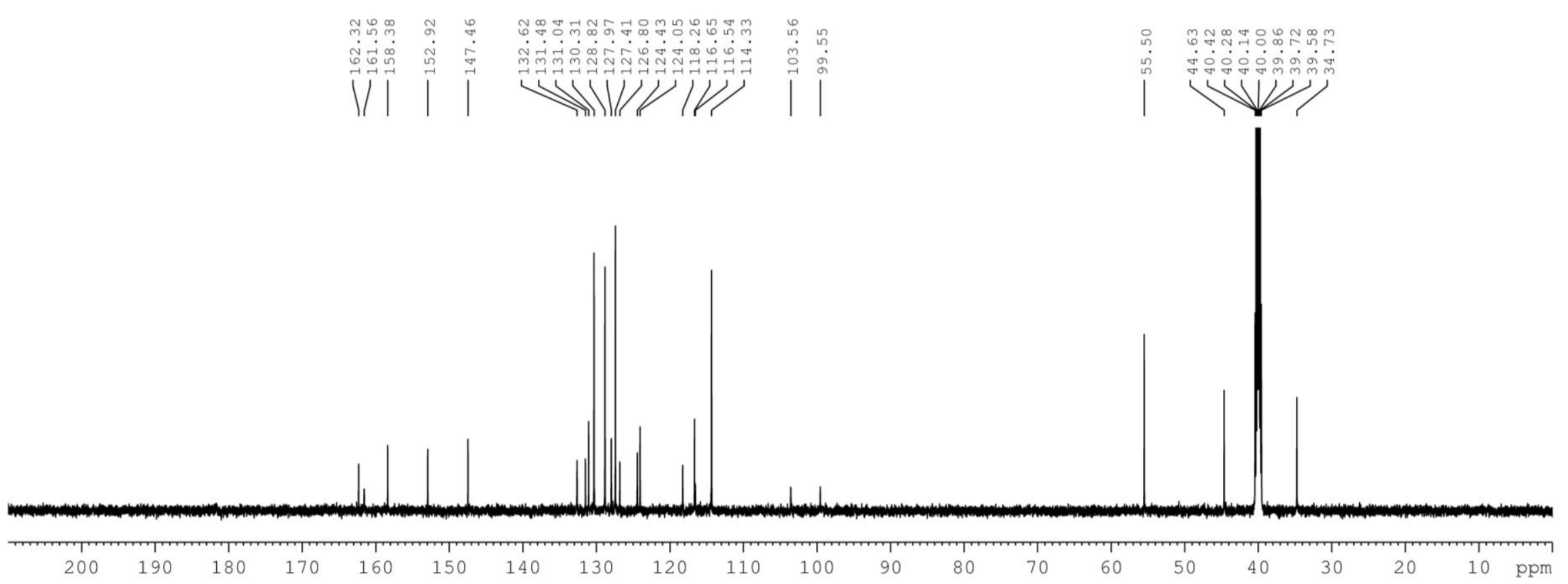

Figure S30. ${ }^{13} \mathrm{C}\left\{{ }^{1} \mathrm{H}\right\}$ NMR $\left(150 \mathrm{MHz}, \mathrm{DMSO}-d_{6}\right)$ spectra of compound 5c 

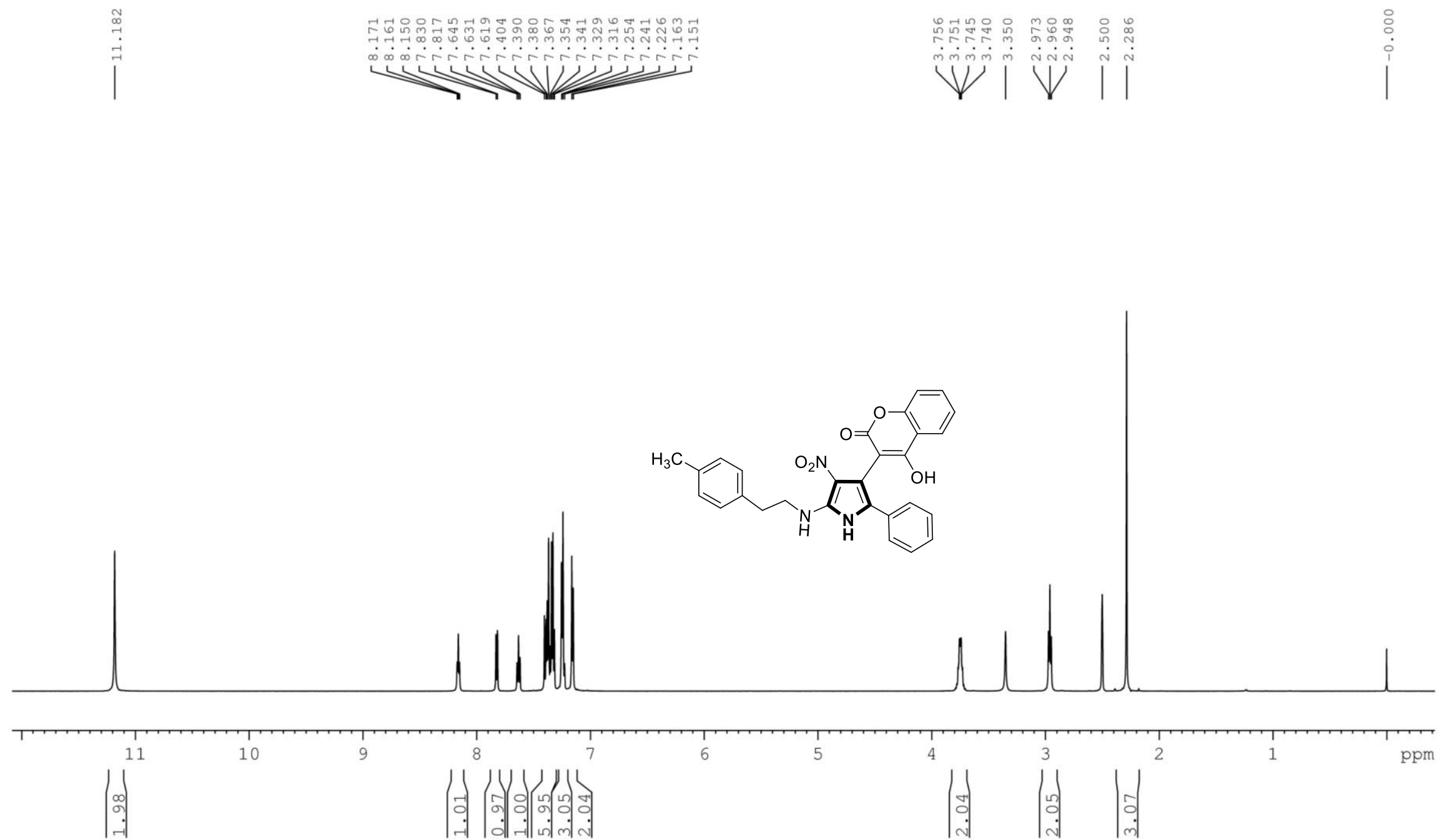

Figure S31. ${ }^{1} \mathrm{H}$ NMR $\left(600 \mathrm{MHz}, \mathrm{DMSO}-d_{6}\right)$ spectra of compound 5d 


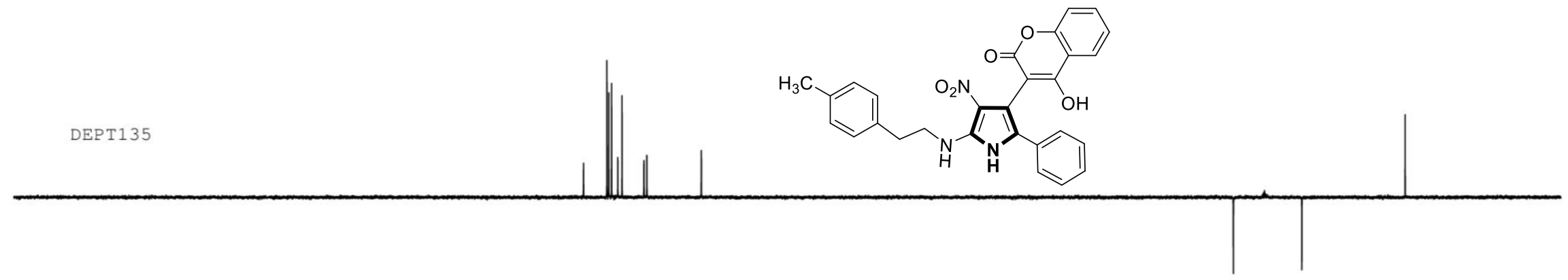

YUNNAN UNIVERSITY ASCEND AVIIIHD600 ZOX-a-56 Jan26-2019-ziquanxing C13CPD DMSO

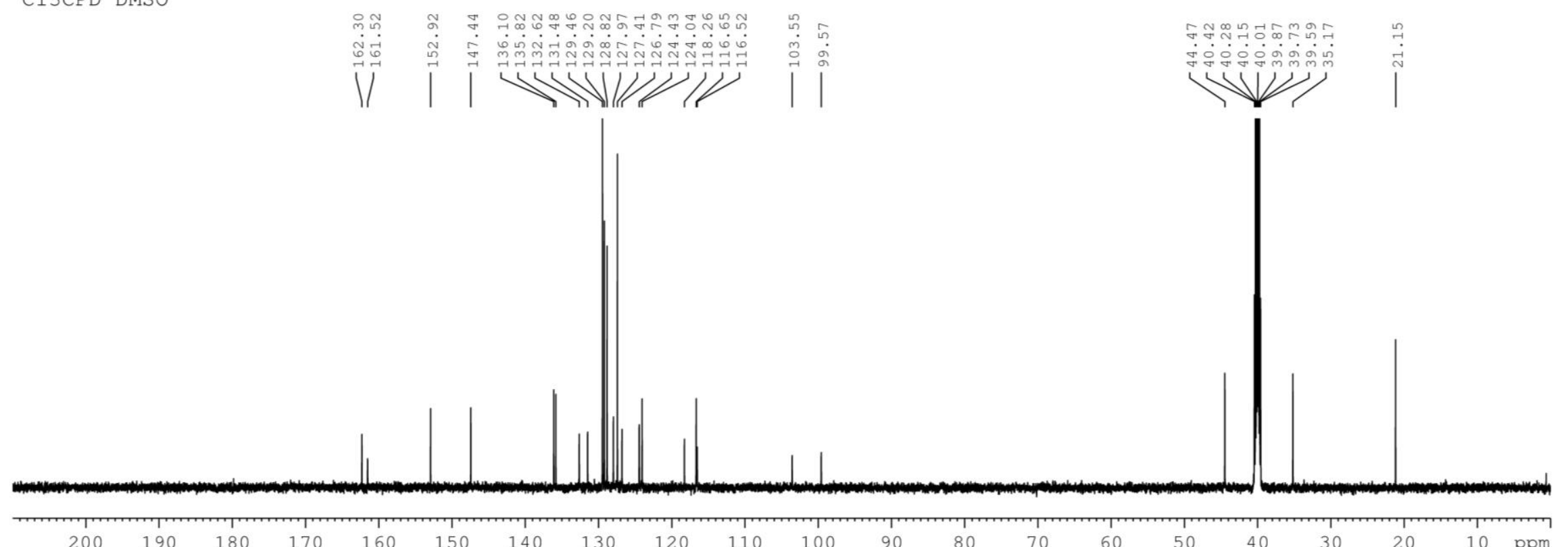

Figure S32. ${ }^{13} \mathrm{C}\left\{{ }^{1} \mathrm{H}\right\}$ NMR $\left(150 \mathrm{MHz}\right.$, DMSO- $\left.d_{6}\right)$ spectra of compound 


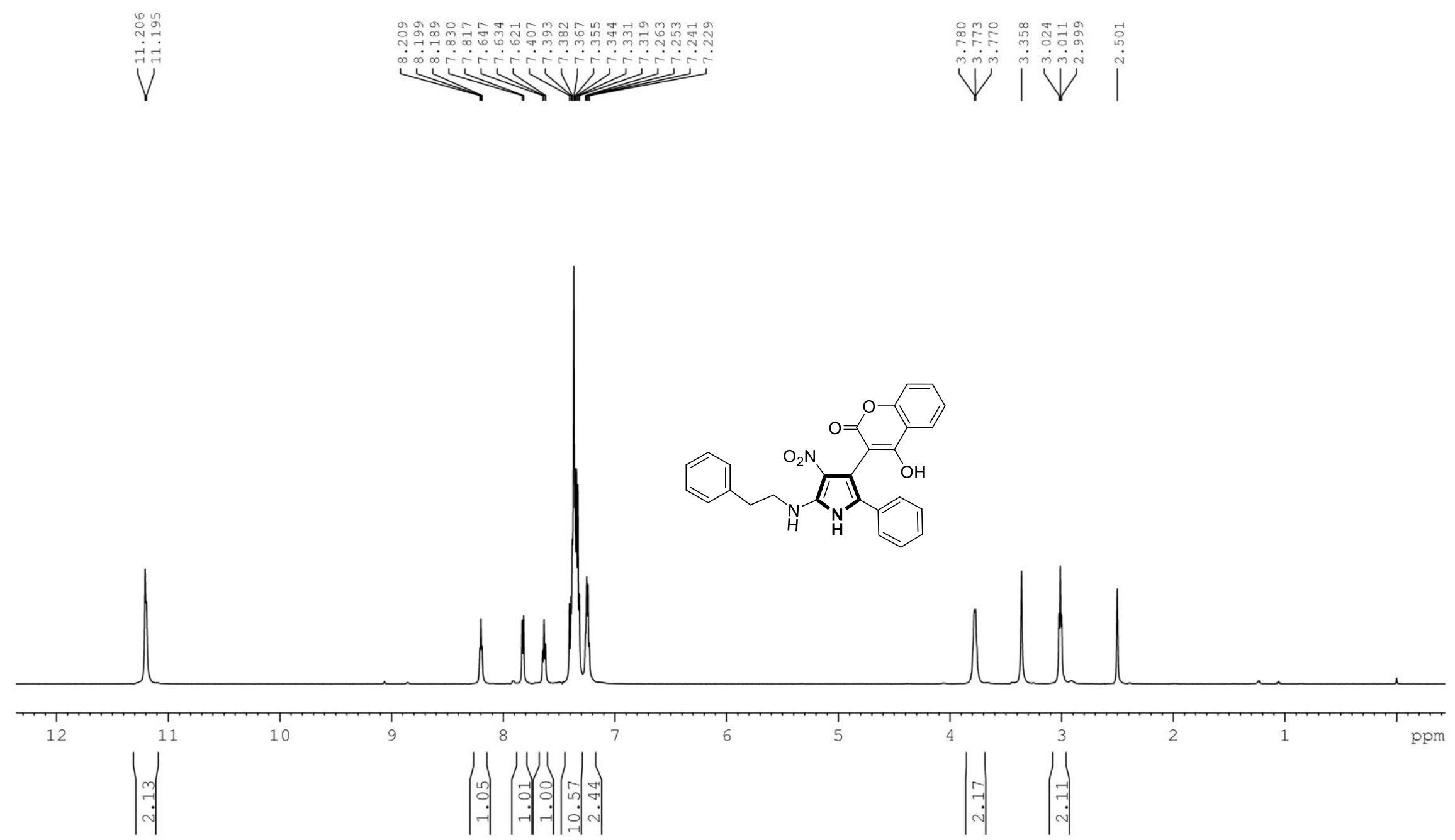

Figure S33. ${ }^{1} \mathrm{H}$ NMR $\left(600 \mathrm{MHz}\right.$, DMSO- $\left.d_{6}\right)$ spectra of compound $\mathbf{5 e}$ 


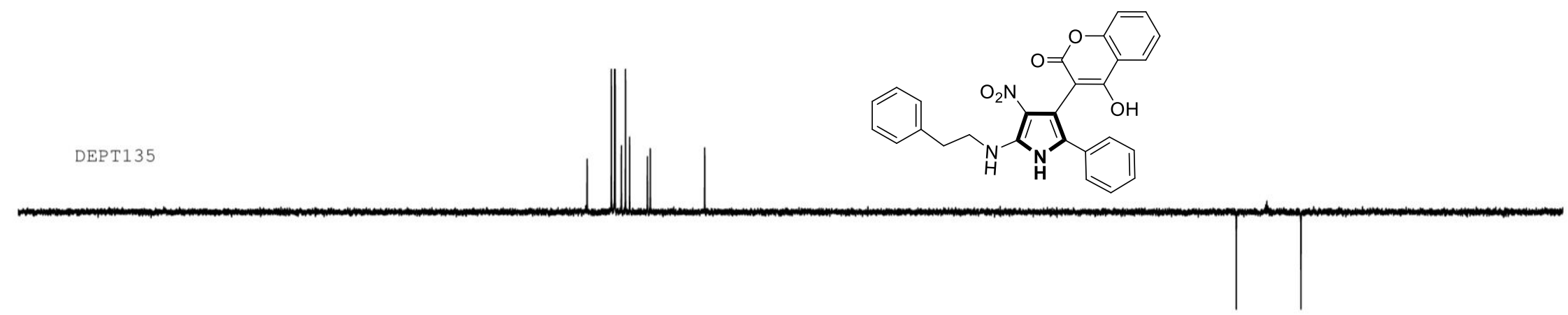

YUNNAN UNIVERSITY ASCEND AVIIIHD600 ZQX-a-43

Jan26-2019-ziquanxing

C13CPD DMSO
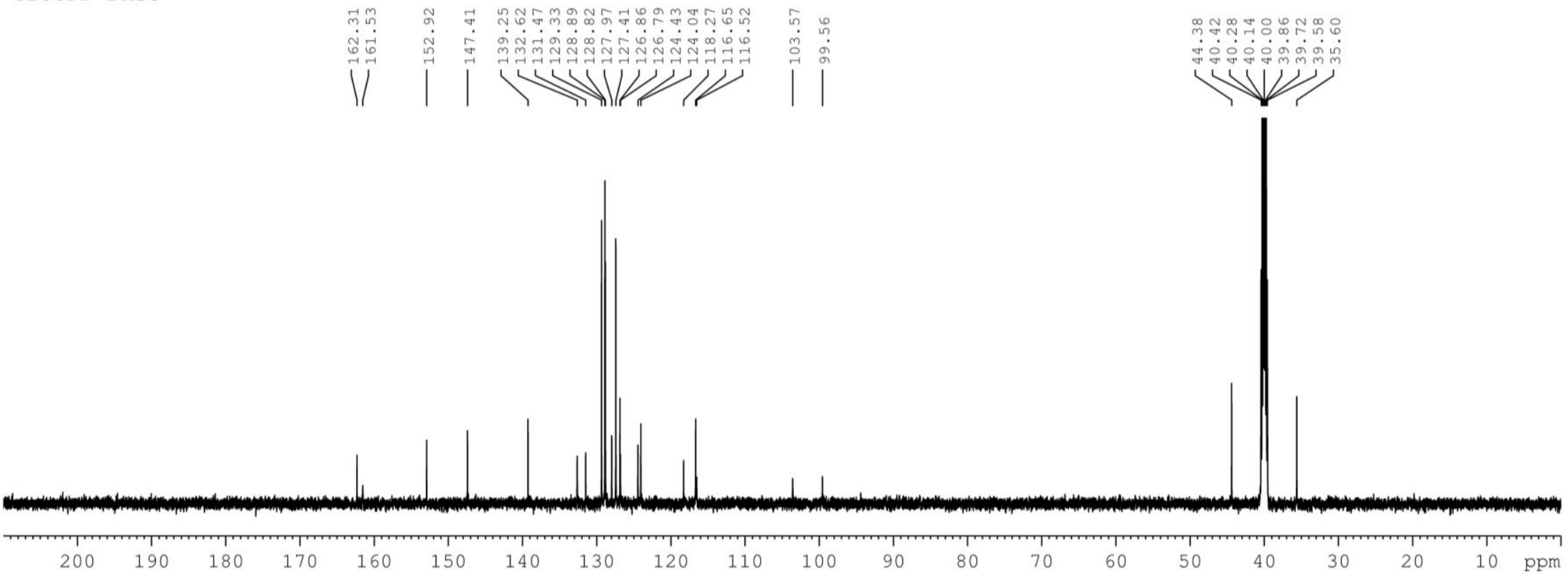

Figure S34. ${ }^{13} \mathrm{C}\left\{{ }^{1} \mathrm{H}\right\}$ NMR $\left(150 \mathrm{MHz}, \mathrm{DMSO}-d_{6}\right)$ spectra of compound $\mathbf{5 e}$ 

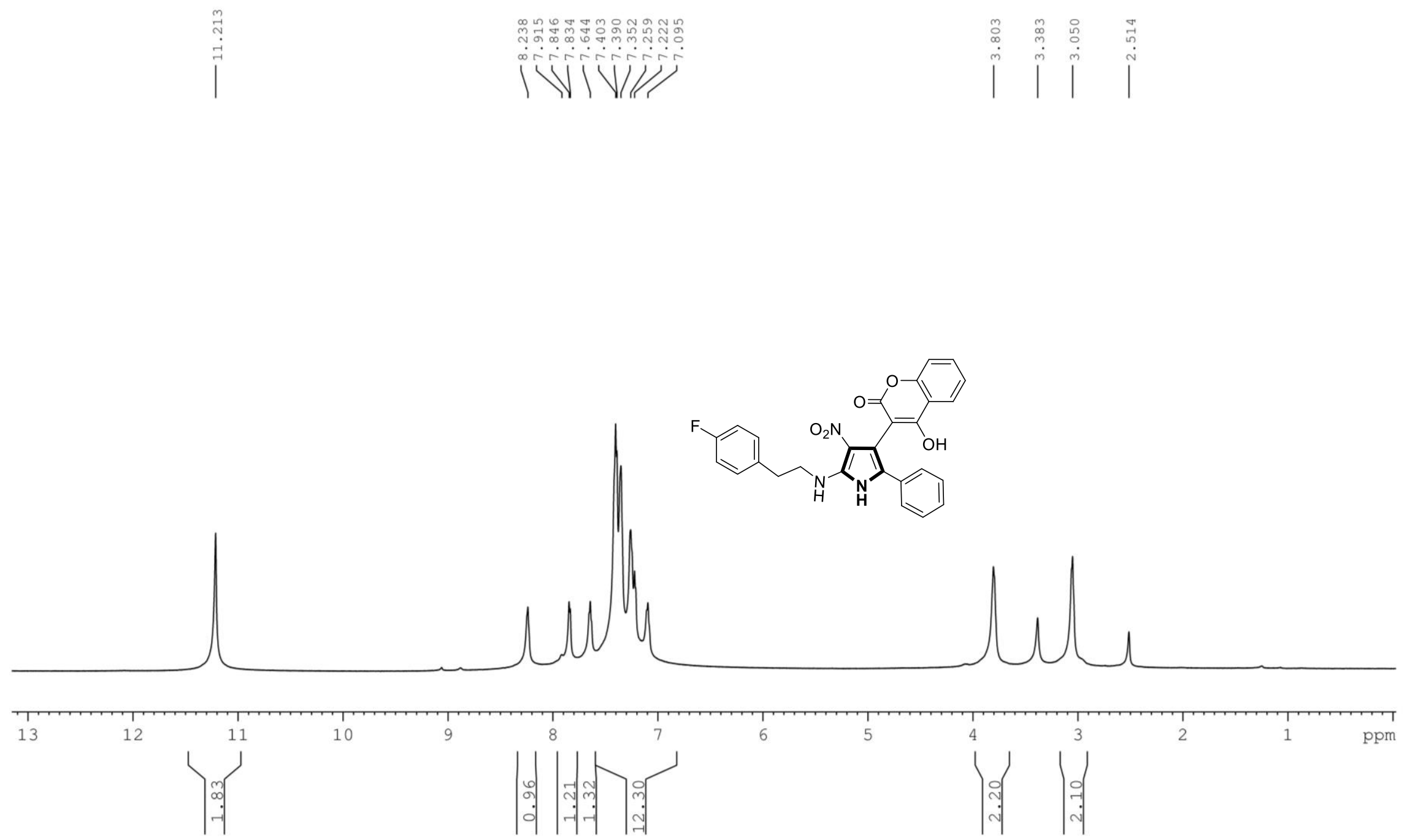

Figure S35. ${ }^{1} \mathrm{H}$ NMR (600 MHz, DMSO- $d_{6}$ ) spectra of compound $\mathbf{5 f}$ 


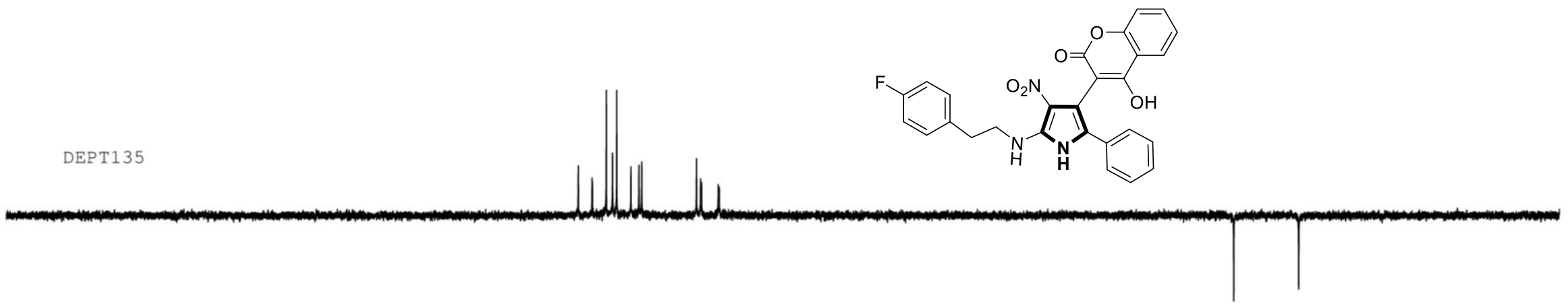

YUNNAN UNIVERSITY ASCEND AVIIIHD600 ZQX-a-45 Jan26-2019-ziquanxing

C13CPD DMSO
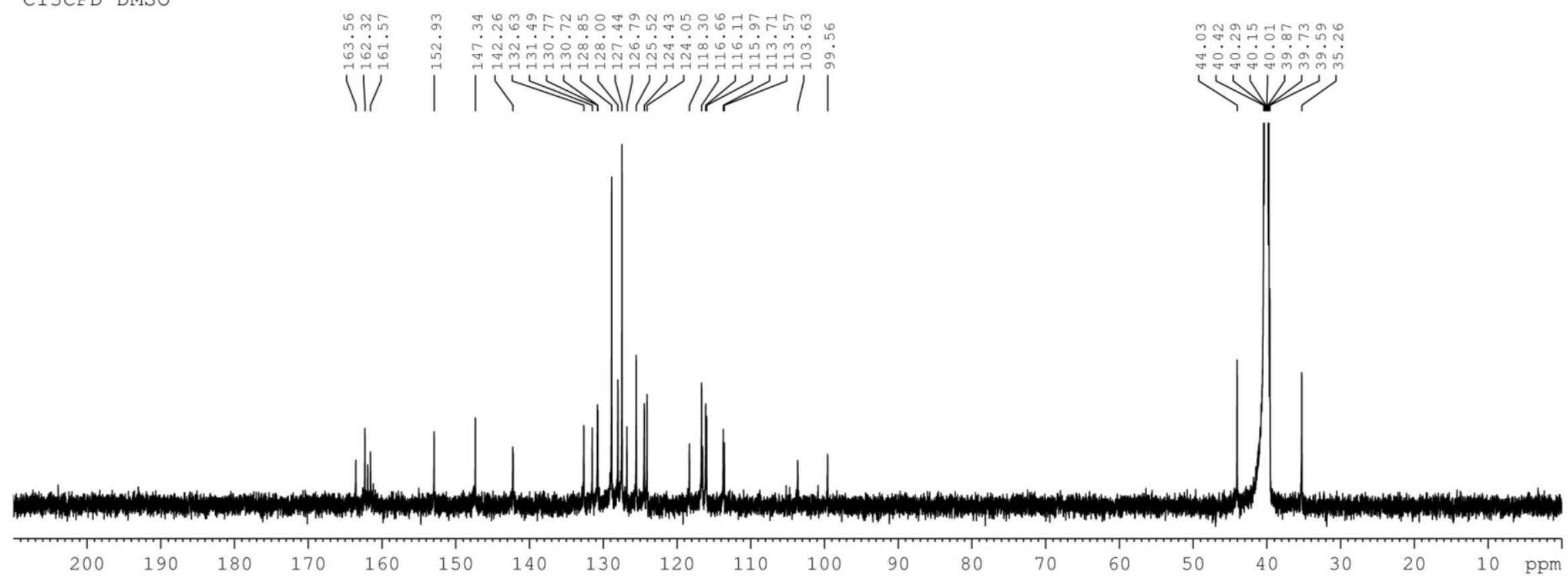

Figure S36. ${ }^{13} \mathrm{C}\left\{{ }^{1} \mathrm{H}\right\}$ NMR (150MHz, DMSO-d $d_{6}$ ) spectra of compound $\mathbf{5 f}$ 

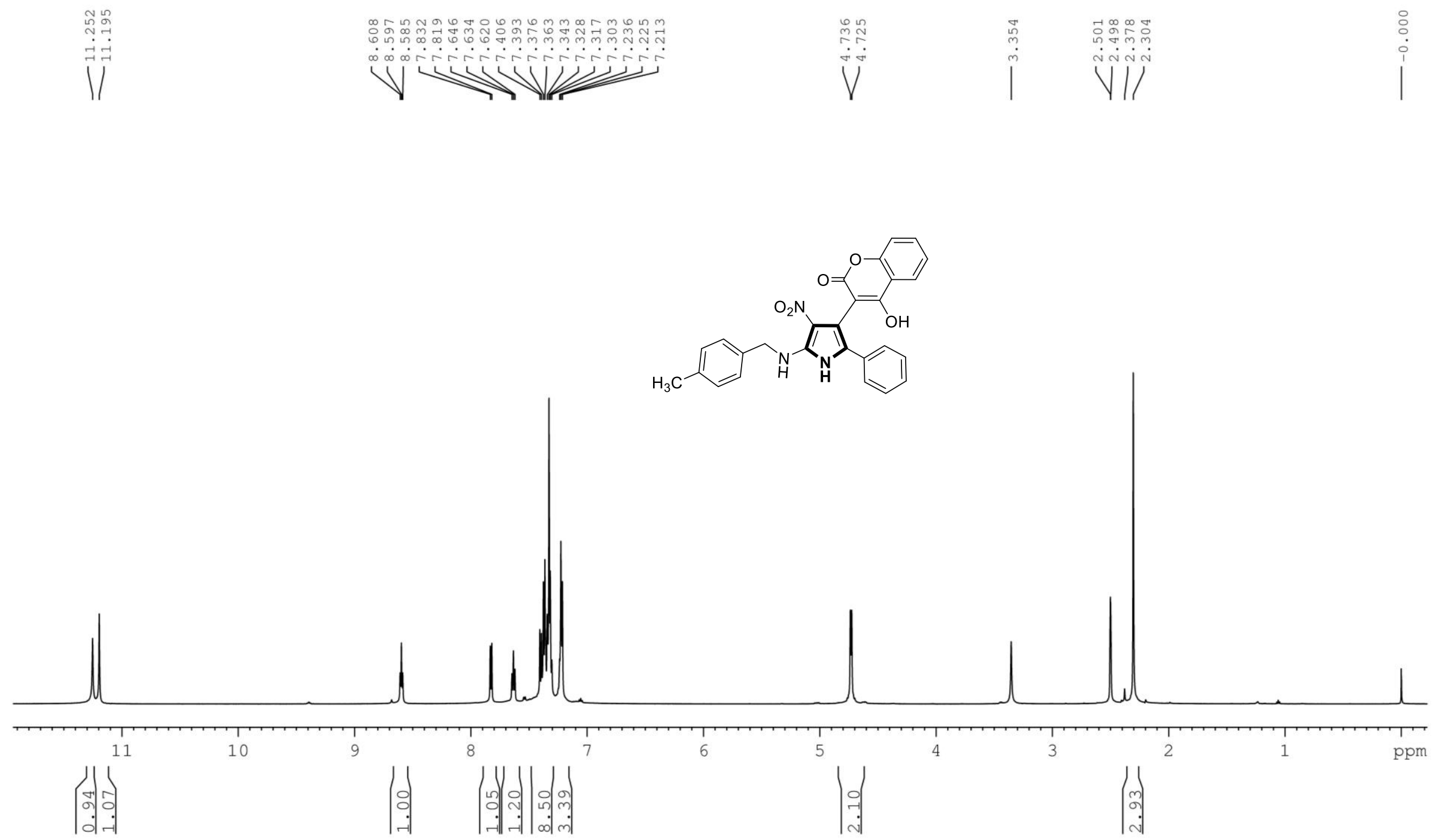

Figure S37. ${ }^{1} \mathrm{H}$ NMR (600 MHz, DMSO- $\left.d_{6}\right)$ spectra of compound $\mathbf{5 g}$ 


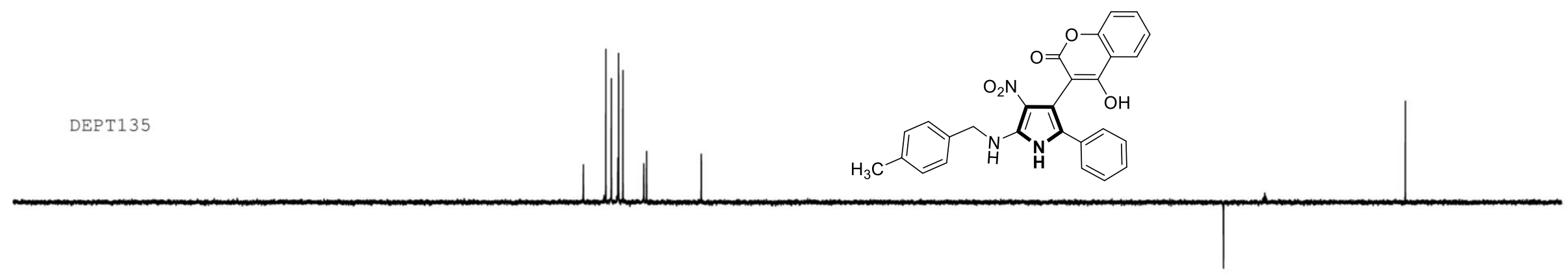

YUNNAN UNIVERSITY ASCEND AVIIIHD600 ZOX-a-55 Jan26-2019-ziquanxing

C13CPD DMSO

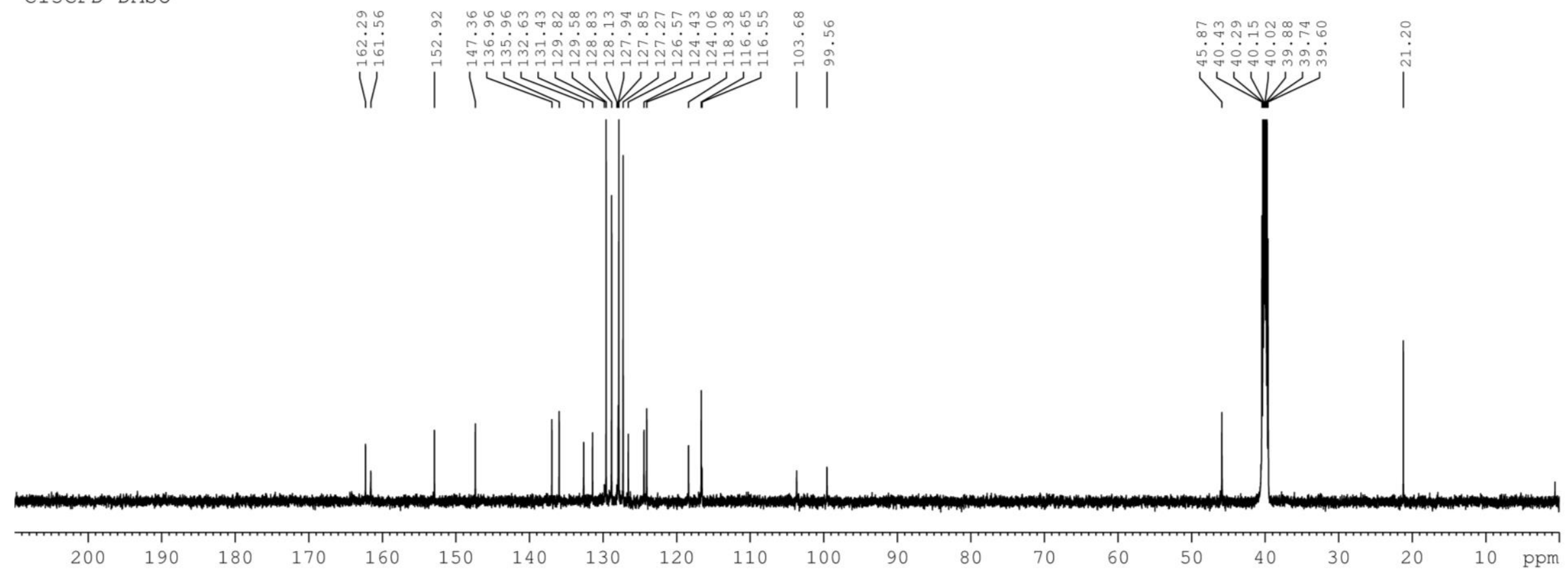

Figure S38. ${ }^{13} \mathrm{C}\left\{{ }^{1} \mathrm{H}\right\}$ NMR $\left(150 \mathrm{MHz}\right.$, DMSO- $\left.d_{6}\right)$ spectra of compound $\mathbf{5 g}$ 

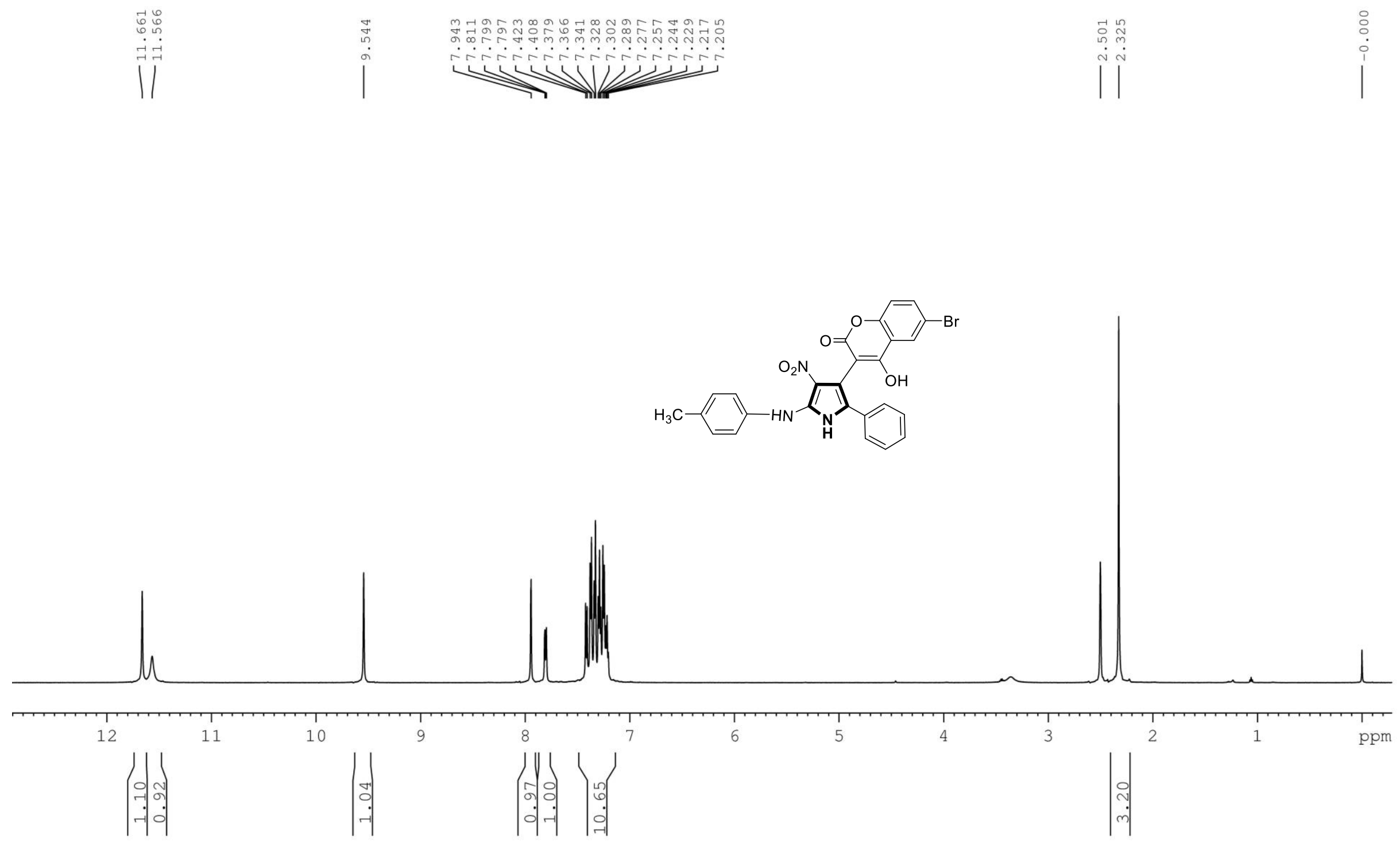

Figure S39. ${ }^{1} \mathrm{H}$ NMR (600 MHz, DMSO- $d_{6}$ ) spectra of compound $\mathbf{5 h}$ 


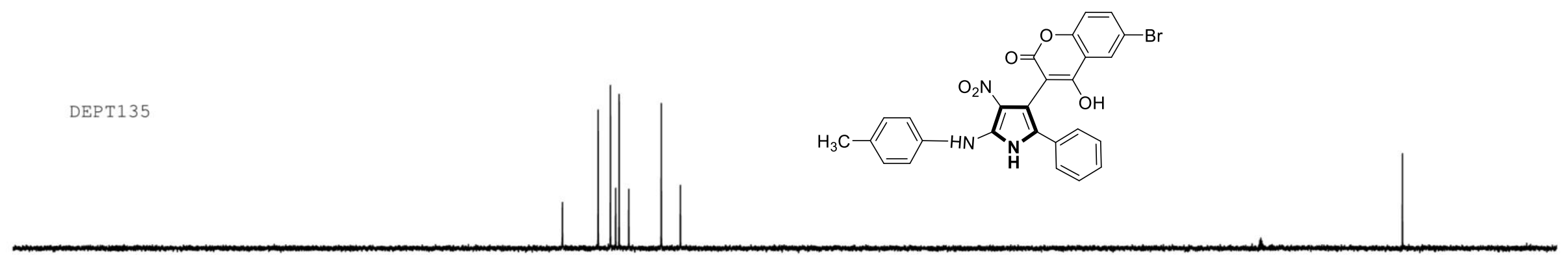

YUNNAN UNIVERSITY ASCEND AVIIIHD600 ZOX-a-50 Jan26-2019-ziquanxing

C13CPD DMSO
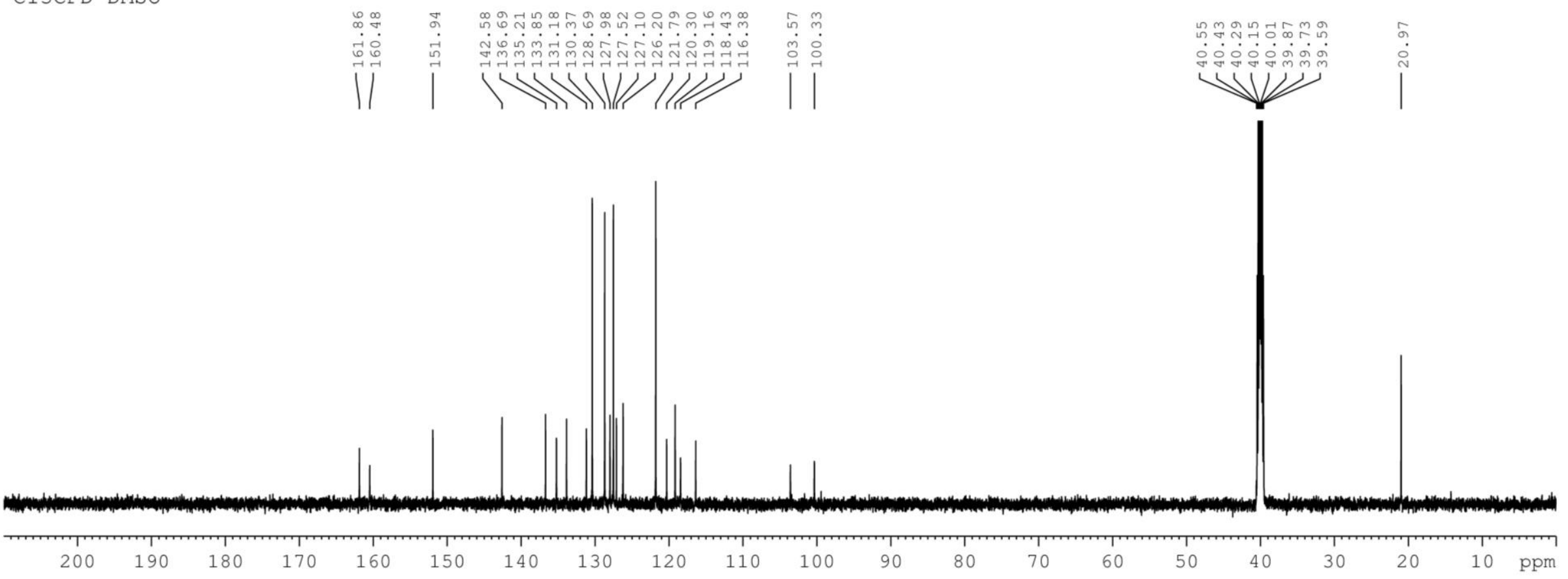

Figure S40. ${ }^{13} \mathrm{C}\left\{{ }^{1} \mathrm{H}\right\}$ NMR $\left(150 \mathrm{MHz}\right.$, DMSO- $\left.d_{6}\right)$ spectra of compound $\mathbf{5 h}$ 


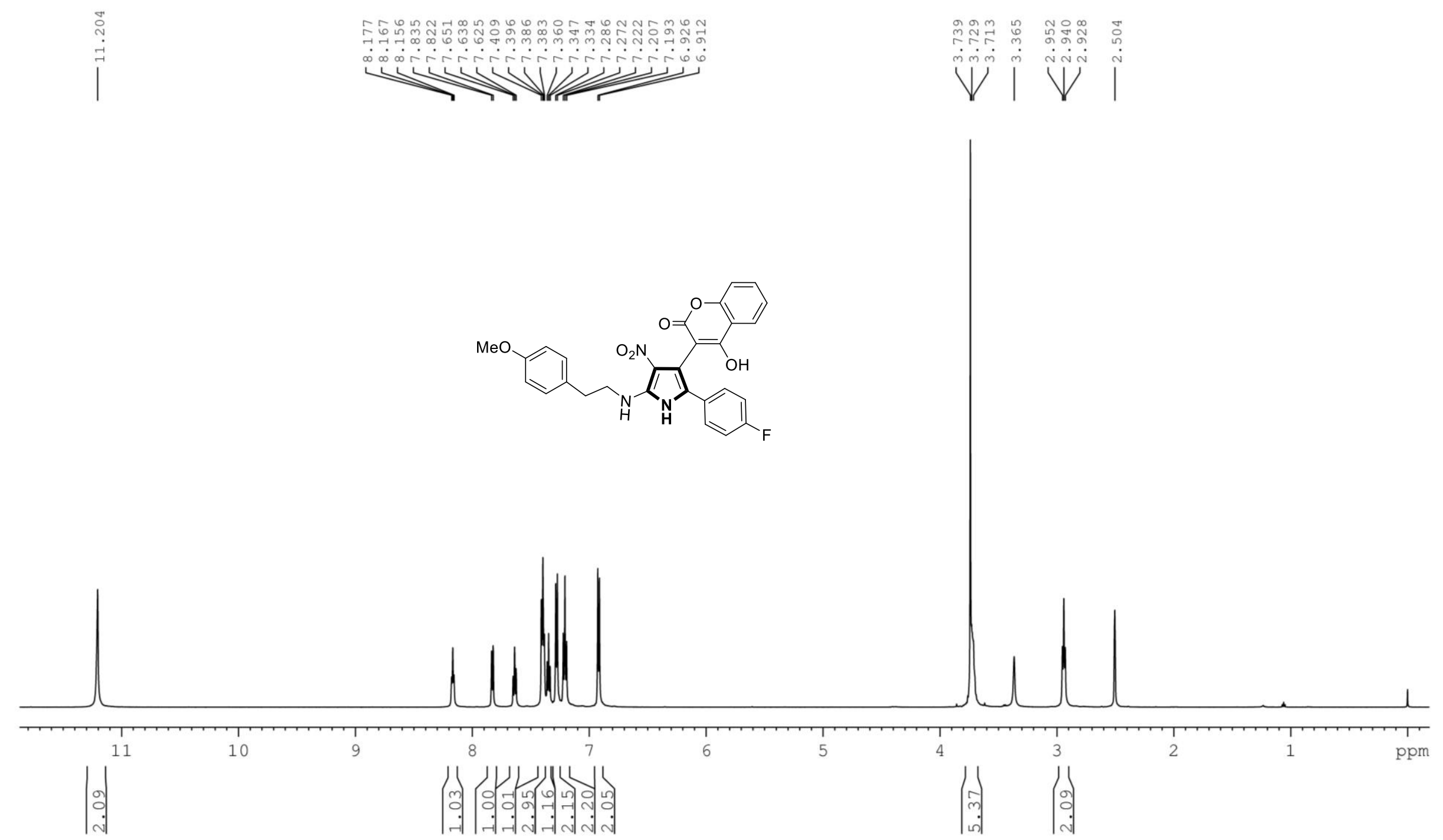

Figure S41. ${ }^{1} \mathrm{H}$ NMR (600 MHz, DMSO-d $\left.d_{6}\right)$ spectra of compound 5i 


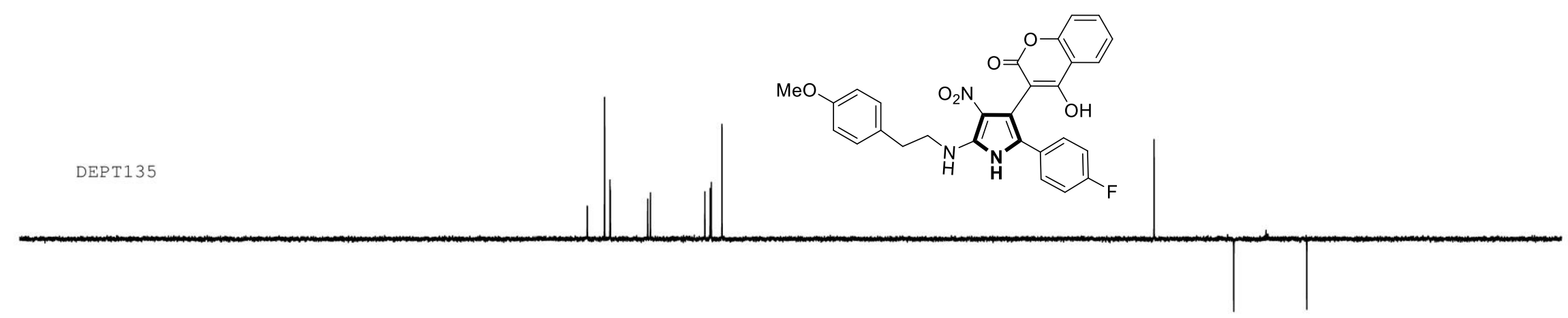

YUNNAN UNIVERSITY ASCEND AVIIIHD600 ZQX-a-57

Jan26-2019-ziquanxing

C13CPD DMSO
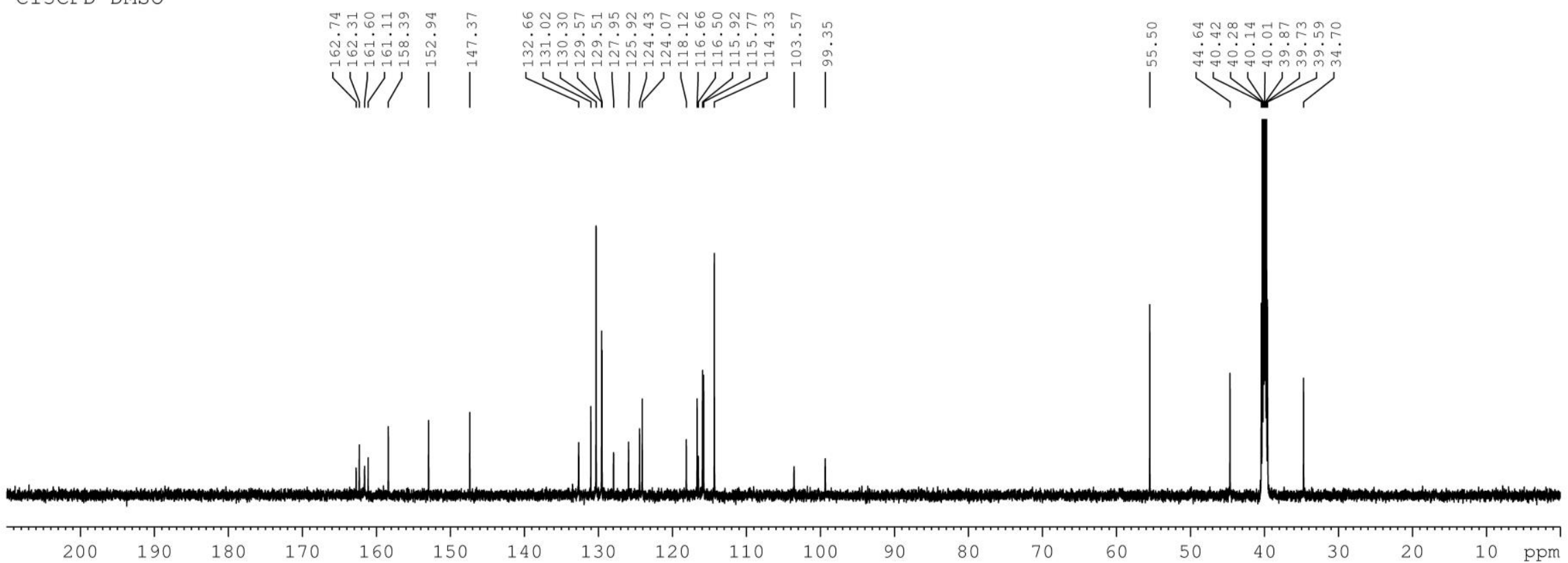

Figure S42. ${ }^{13} \mathrm{C}\left\{{ }^{1} \mathrm{H}\right\}$ NMR $\left(150 \mathrm{MHz}\right.$, DMSO- $\left.d_{6}\right)$ spectra of compound $\mathbf{5 i}$ 


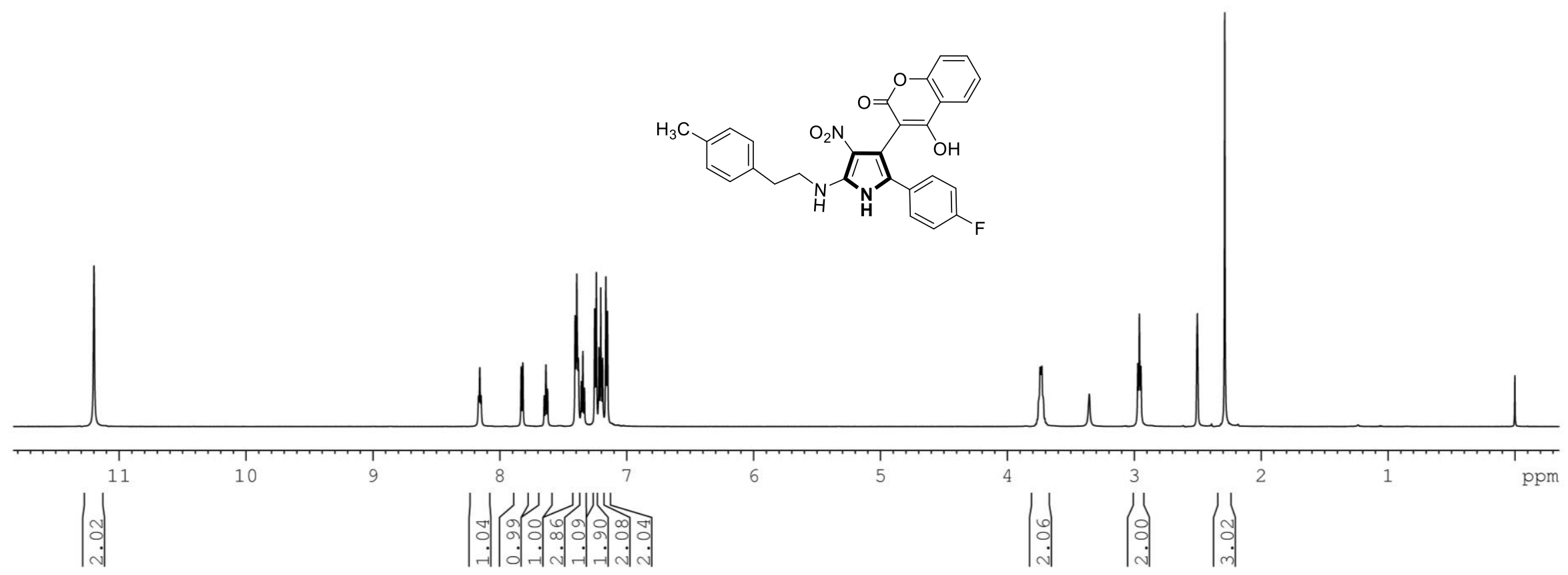

Figure S43. ${ }^{1} \mathrm{H}$ NMR (600 MHz, DMSO-d $)$ spectra of compound 5j 


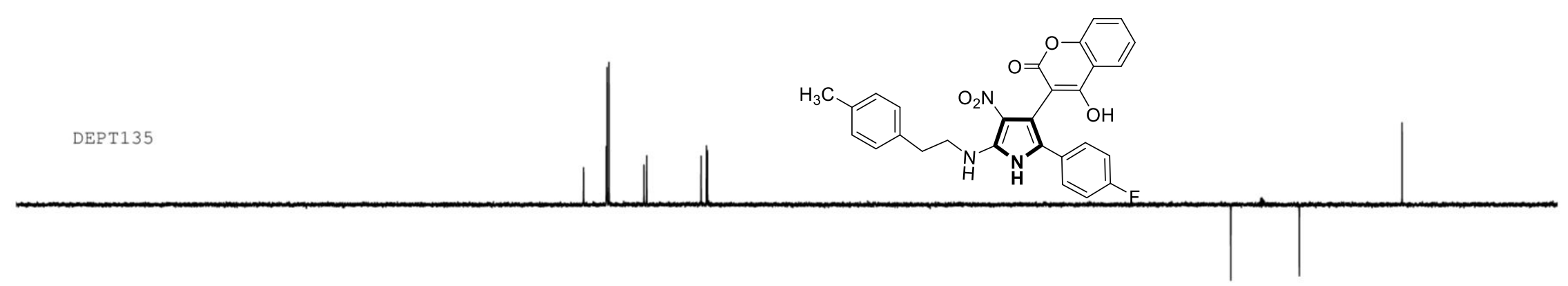

YUNNAN UNIVERSITY ASCEND AVIIIHD600 ZQX-a-52 Jan26-2019-ziquanxing C13CPD DMSO
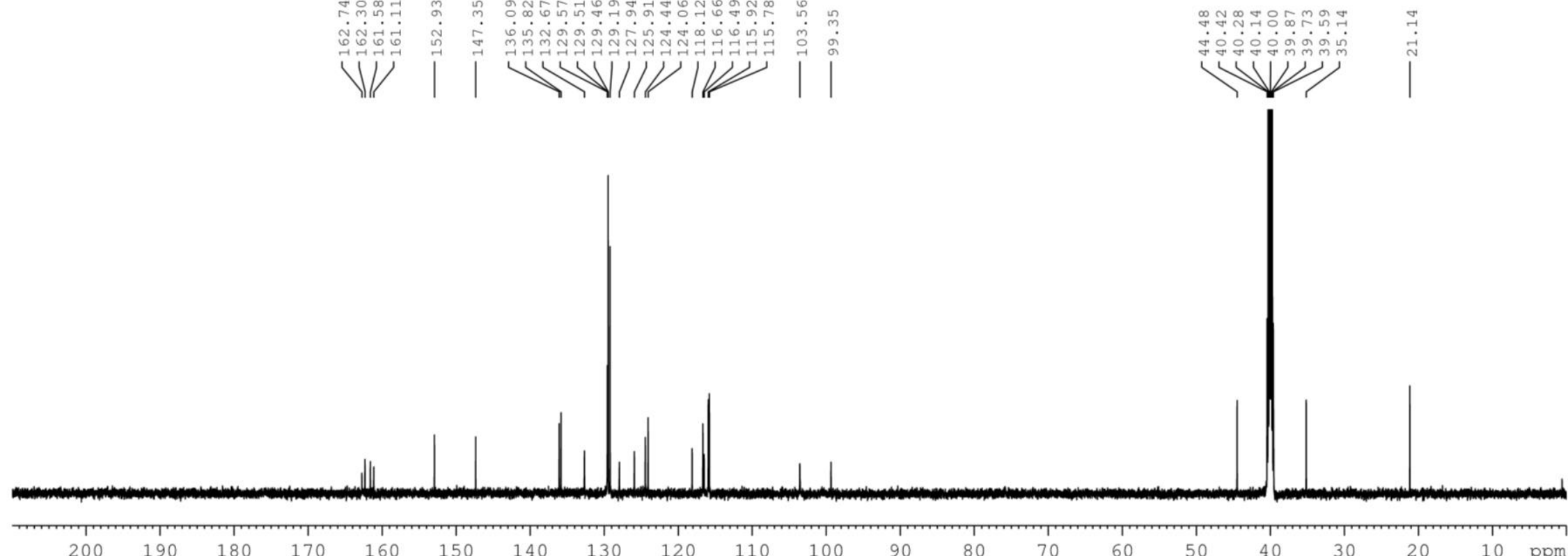

Figure S44. ${ }^{13} \mathrm{C}\left\{{ }^{1} \mathrm{H}\right\}$ NMR $\left(150 \mathrm{MHz}\right.$, DMSO- $\left.d_{6}\right)$ spectra of compound $\mathbf{5 j}$ 


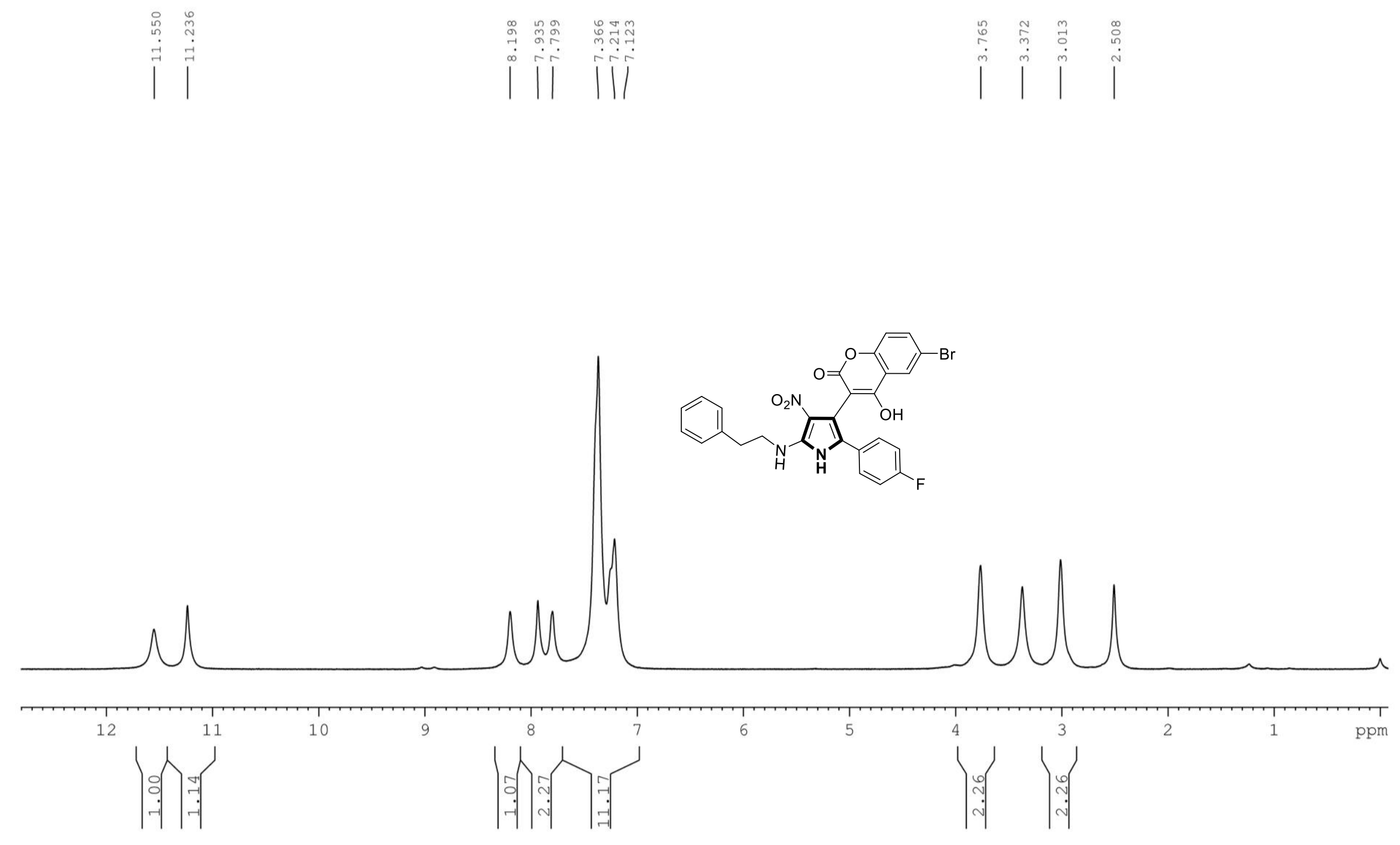

Figure S45. ${ }^{1} \mathrm{H}$ NMR (600 MHz, DMSO-d $)$ spectra of compound $\mathbf{5 k}$ 


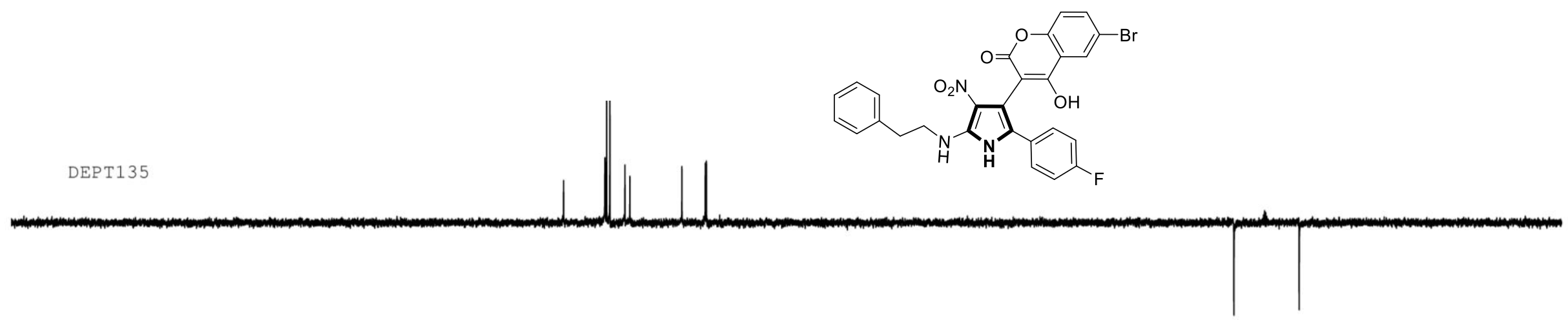

YUNNAN UNIVERSITY ASCEND AVIIIHD600 ZQX-a-49 Jan26-2019-ziquanxing

C13CPD DMSO
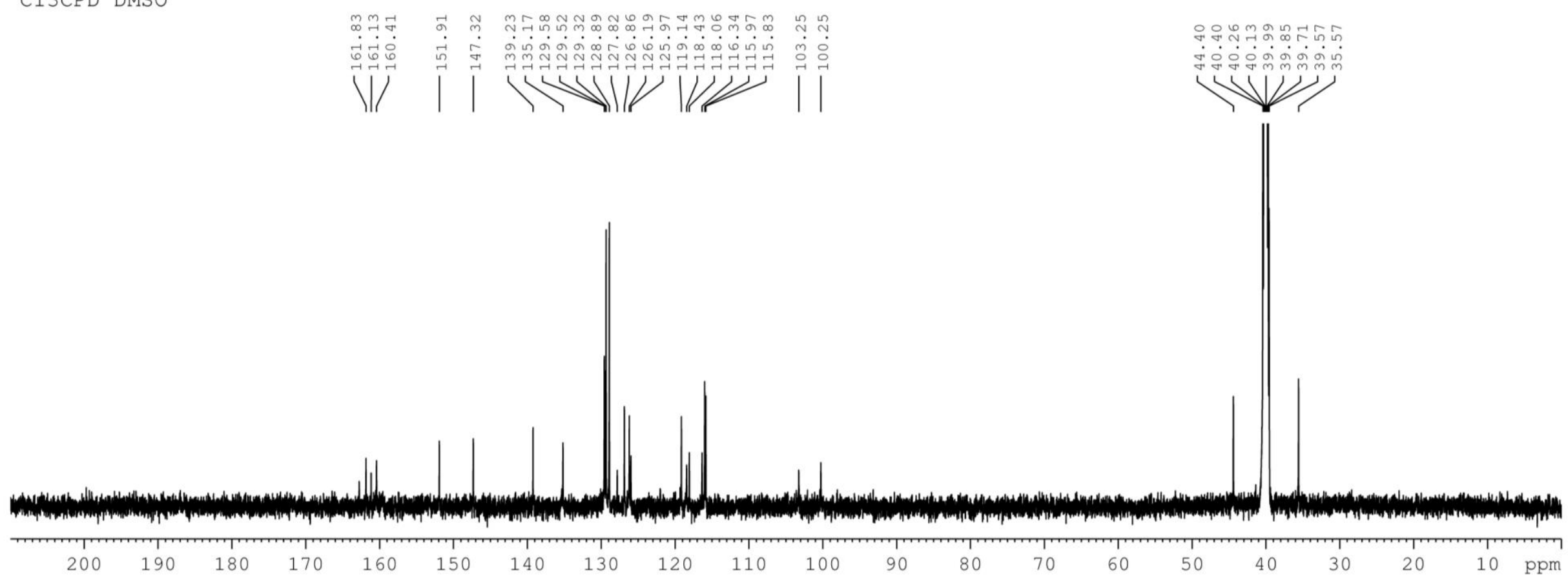

Figure S46. ${ }^{13} \mathrm{C}\left\{{ }^{1} \mathrm{H}\right\}$ NMR $\left(150 \mathrm{MHz}\right.$, DMSO- $\left.d_{6}\right)$ spectra of compound $\mathbf{5 k}$ 


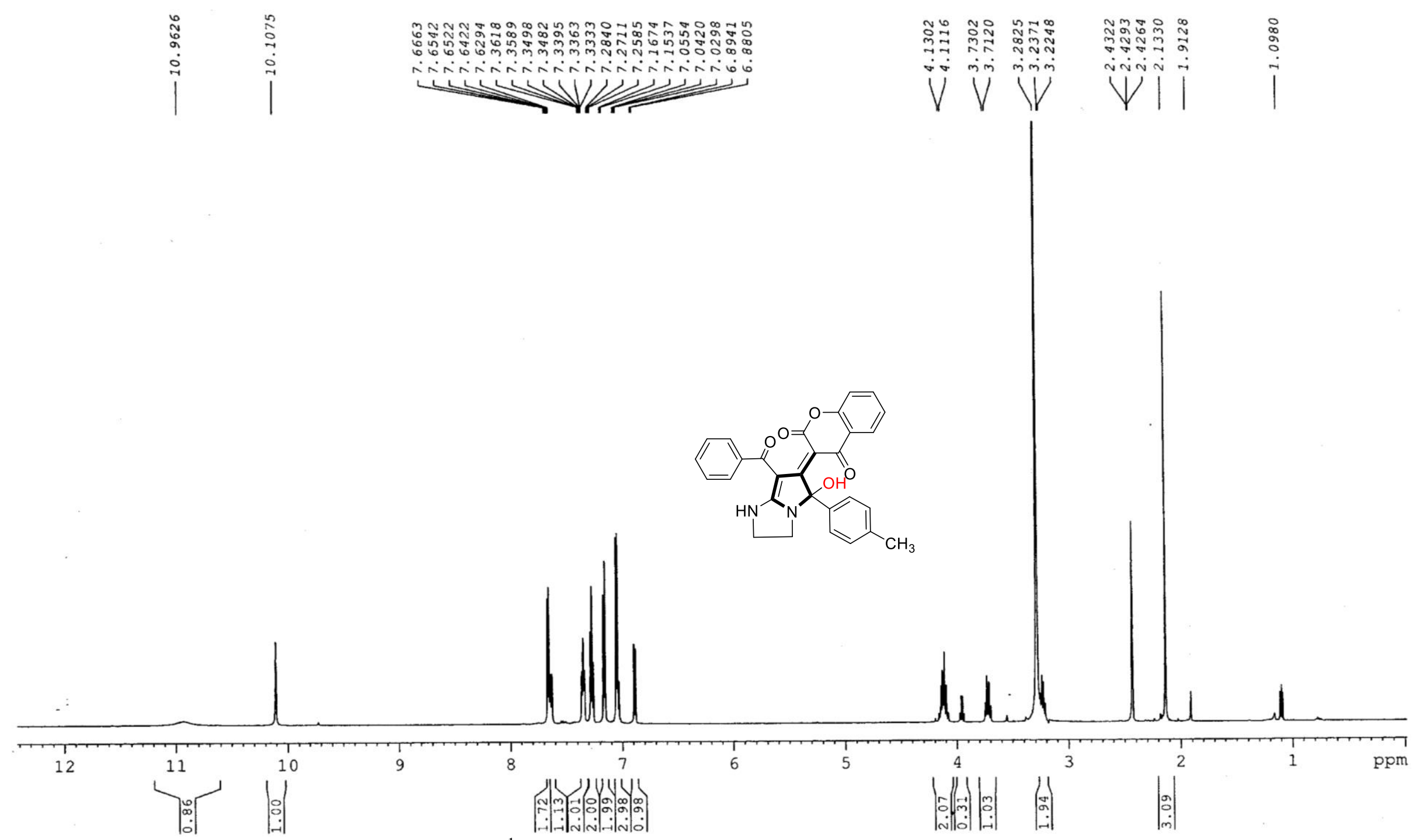

Figure S47. ${ }^{1} \mathrm{H}$ NMR $\left(600 \mathrm{MHz}, \mathrm{DMSO}-d_{6}\right)$ spectra of compound $\mathbf{6 a}$ 


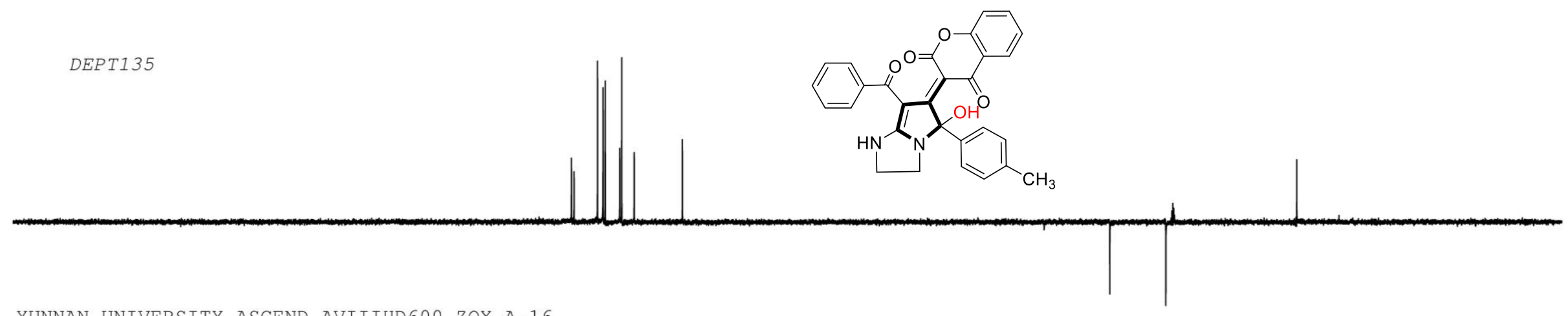

YUNNAN UNIVERSITY ASCEND AVIIIHD600 ZQX-A-16 Sep05-2018-yangchanglong

C13CPD DMSO
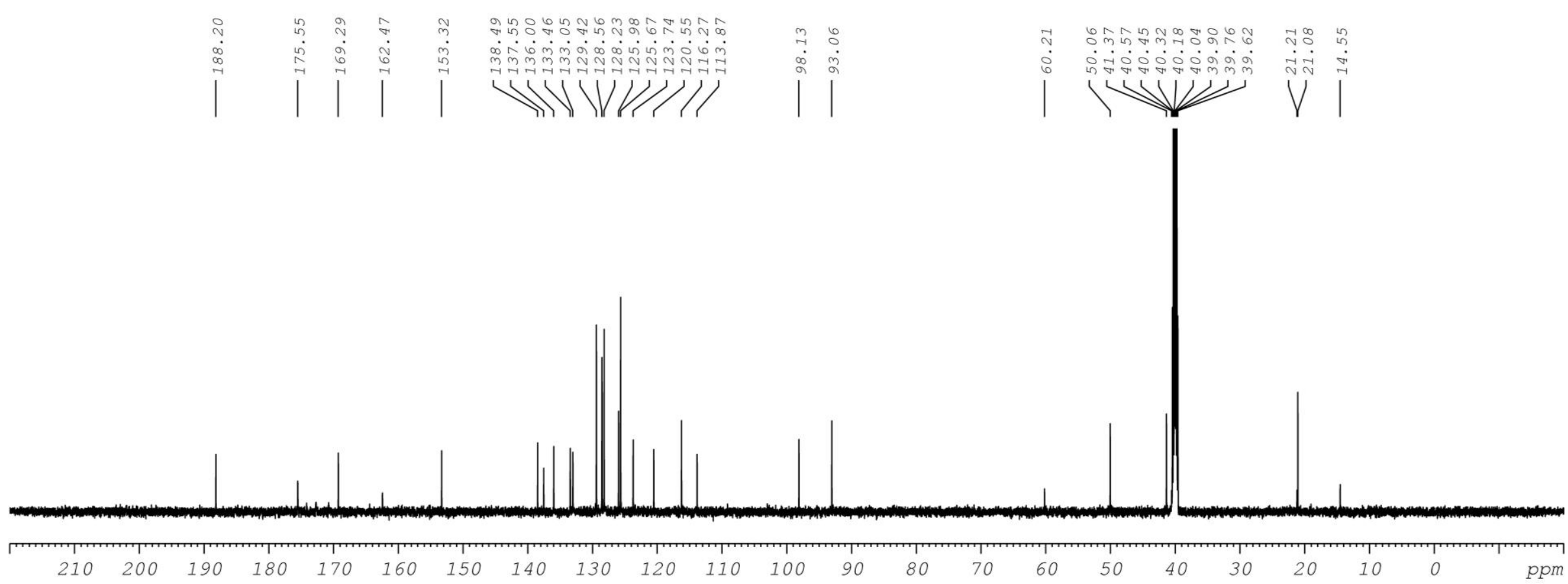

Figure S48. ${ }^{13} \mathrm{C}\left\{{ }^{1} \mathrm{H}\right\}$ NMR (150 MHz, DMSO-d $)$ spectra of compound $6 \mathbf{a}$ 

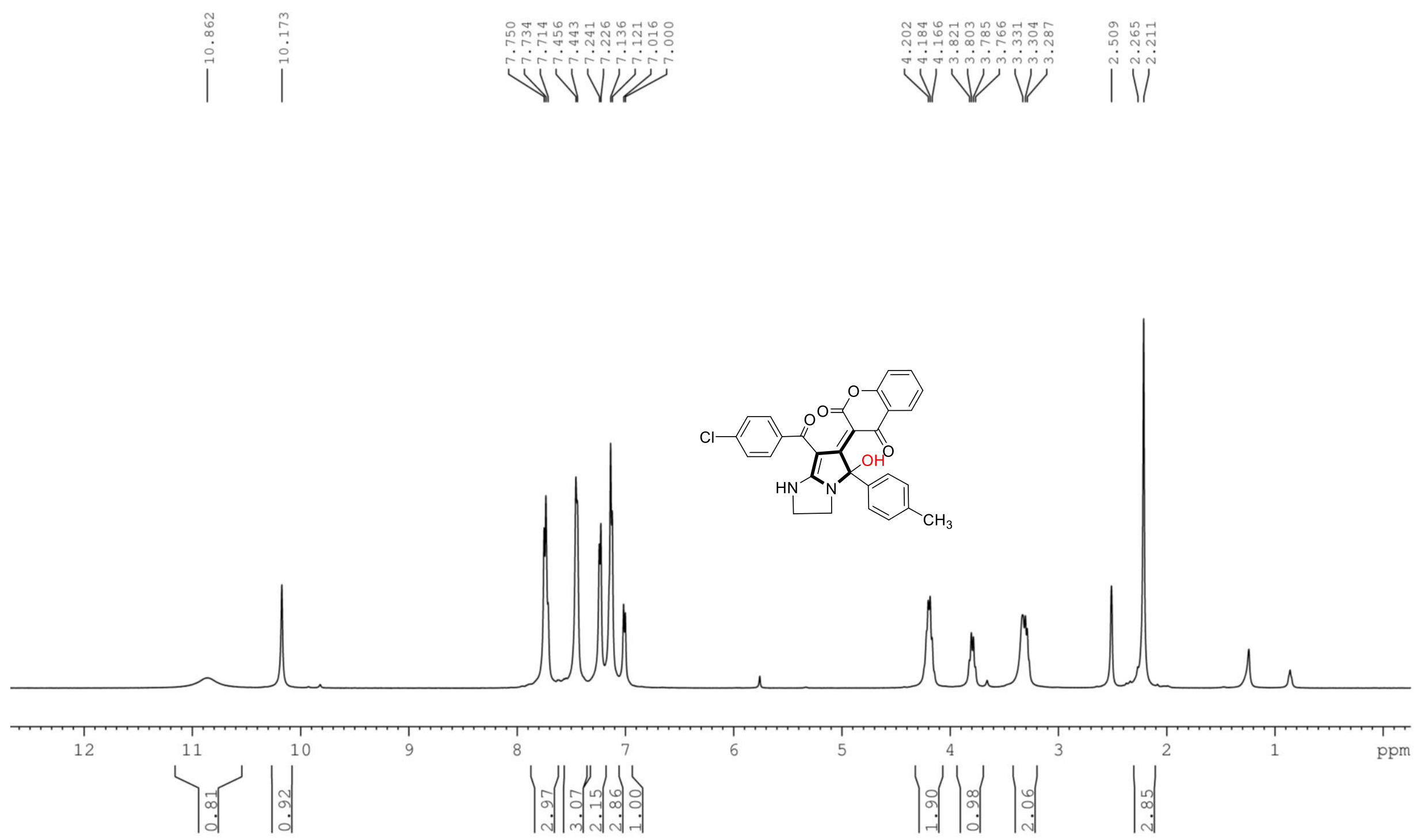

Figure S49. ${ }^{1} \mathrm{H}$ NMR $\left(500 \mathrm{MHz}, \mathrm{DMSO}-d_{6}\right)$ spectra of compound $\mathbf{6 b}$ 


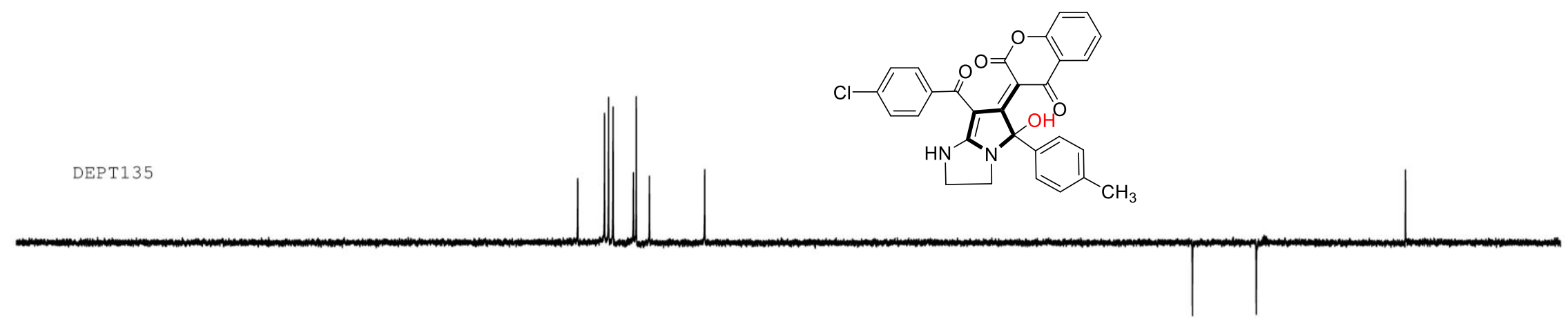

YUNNAN UNIVERSITY AVIIIHD500 ZQX-a-8 Jul06-2018-ziquanxing C13CPD DMSO

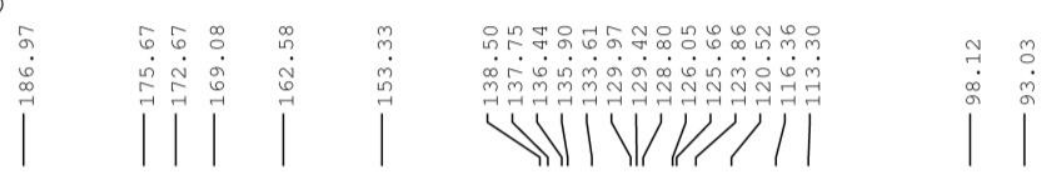

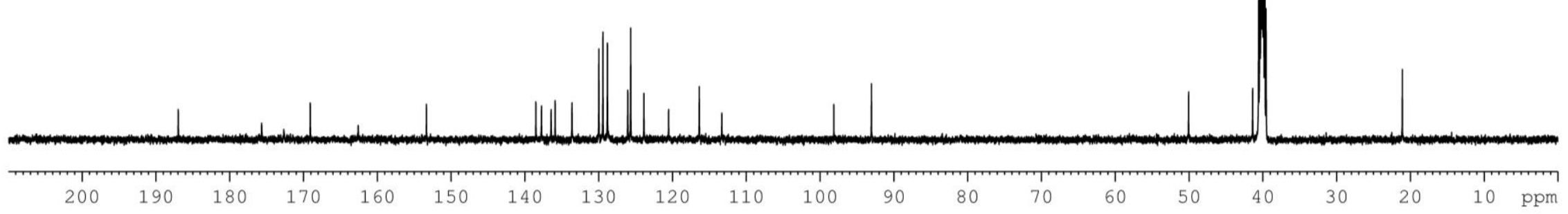

Figure S50. ${ }^{13} \mathrm{C}\left\{{ }^{1} \mathrm{H}\right\}$ NMR $\left(125 \mathrm{MHz}, \mathrm{DMSO}-d_{6}\right)$ spectra of compound $\mathbf{6 b}$ 


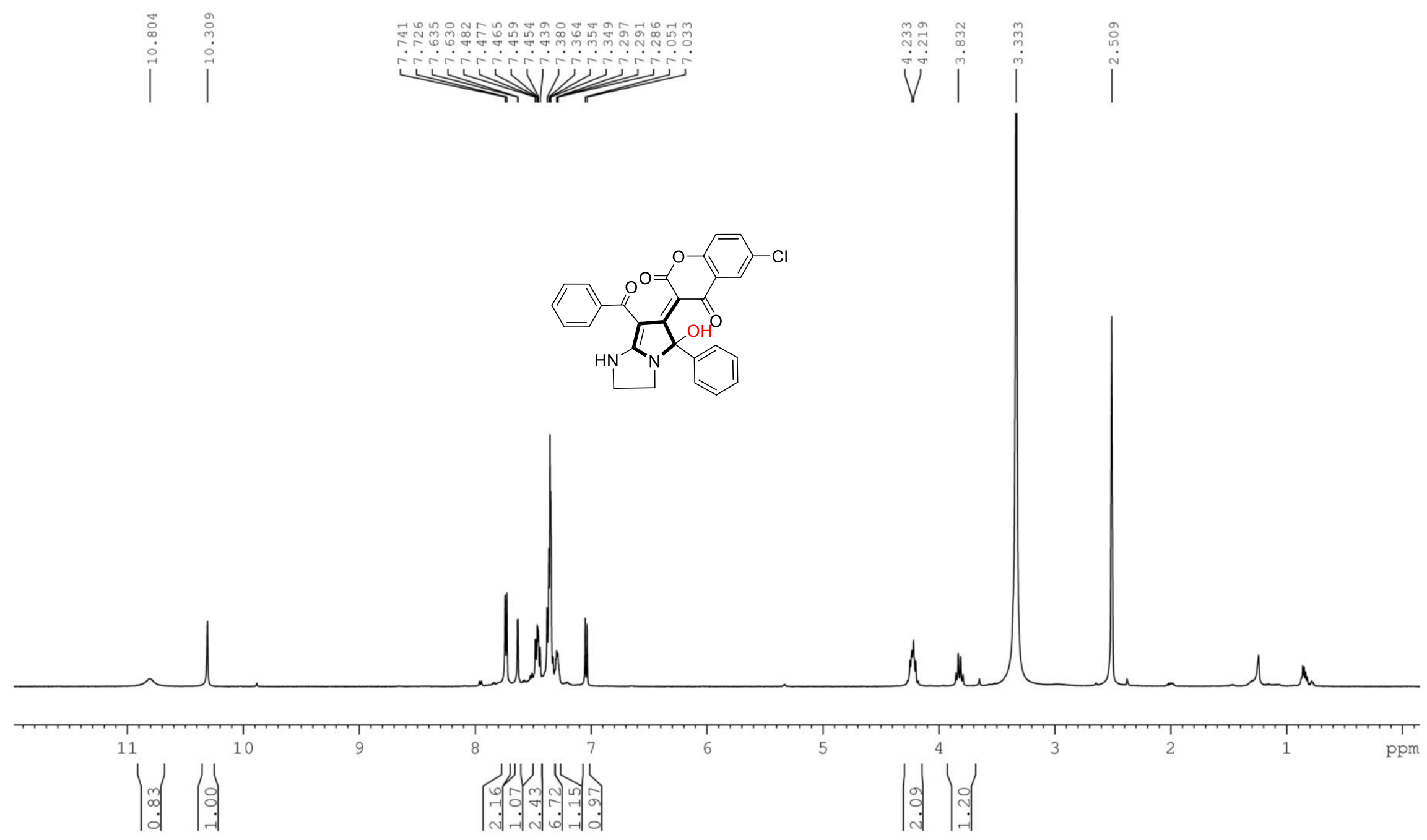

Figure S51. ${ }^{1} \mathrm{H}$ NMR $\left(500 \mathrm{MHz}, \mathrm{DMSO}-d_{6}\right)$ spectra of compound $\mathbf{6 c}$ 


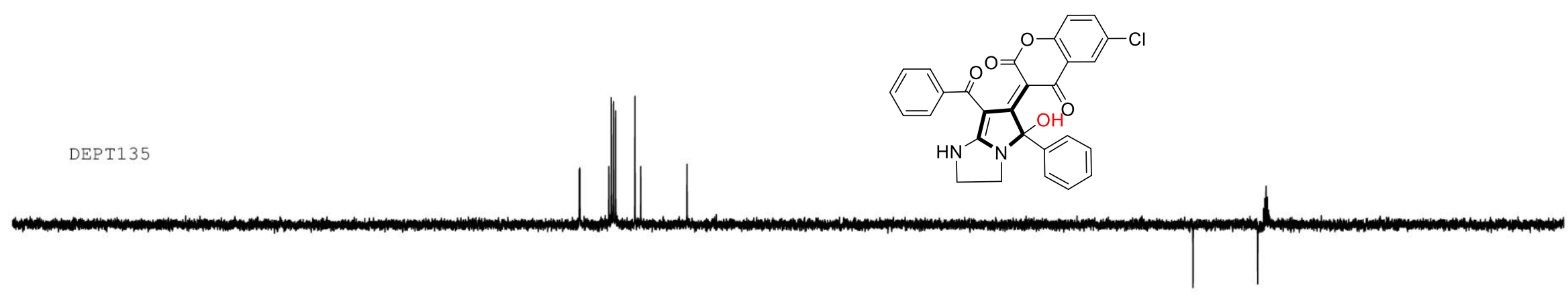

YUNNAN UNIVERSITY AVIIIHD500 ZQX-a-26 Aug06-2018-ziquanxing

C13CPD DMS

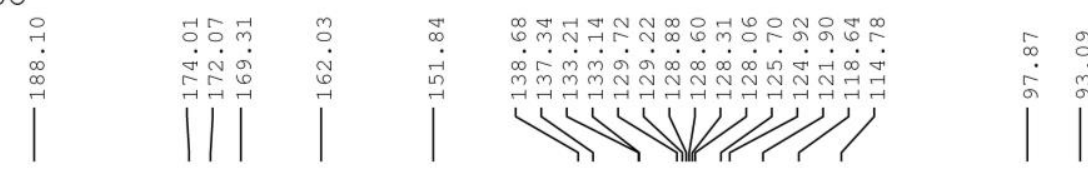

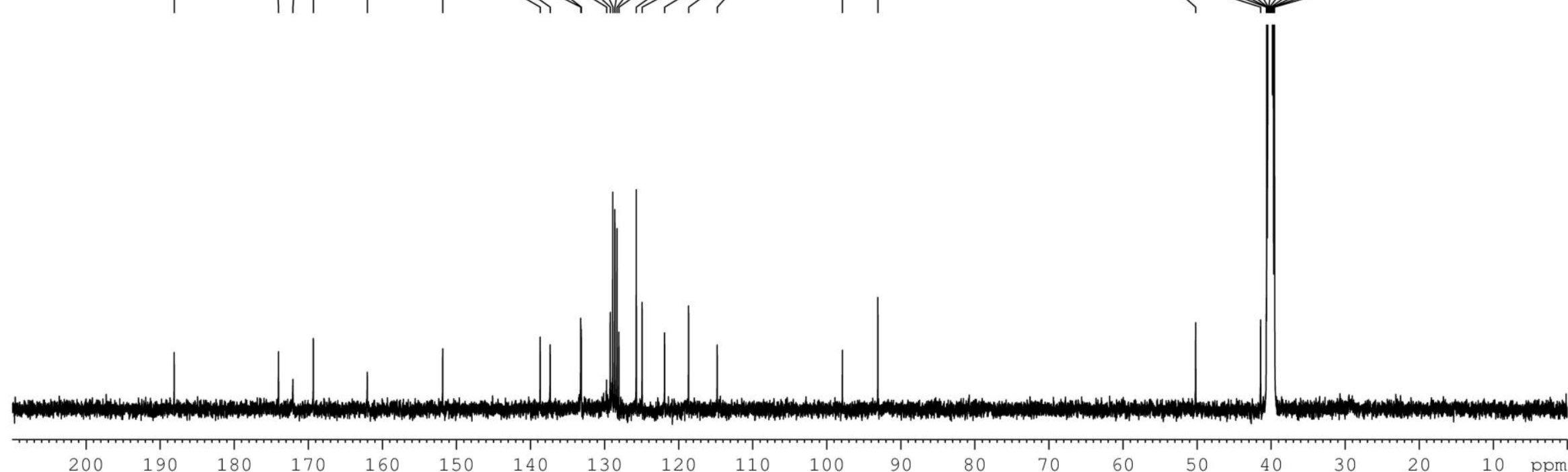

Figure S52. ${ }^{13} \mathrm{C}\left\{{ }^{1} \mathrm{H}\right\}$ NMR (125 MHz, DMSO- $\left.d_{6}\right)$ spectra of compound $\mathbf{6 c}$ 


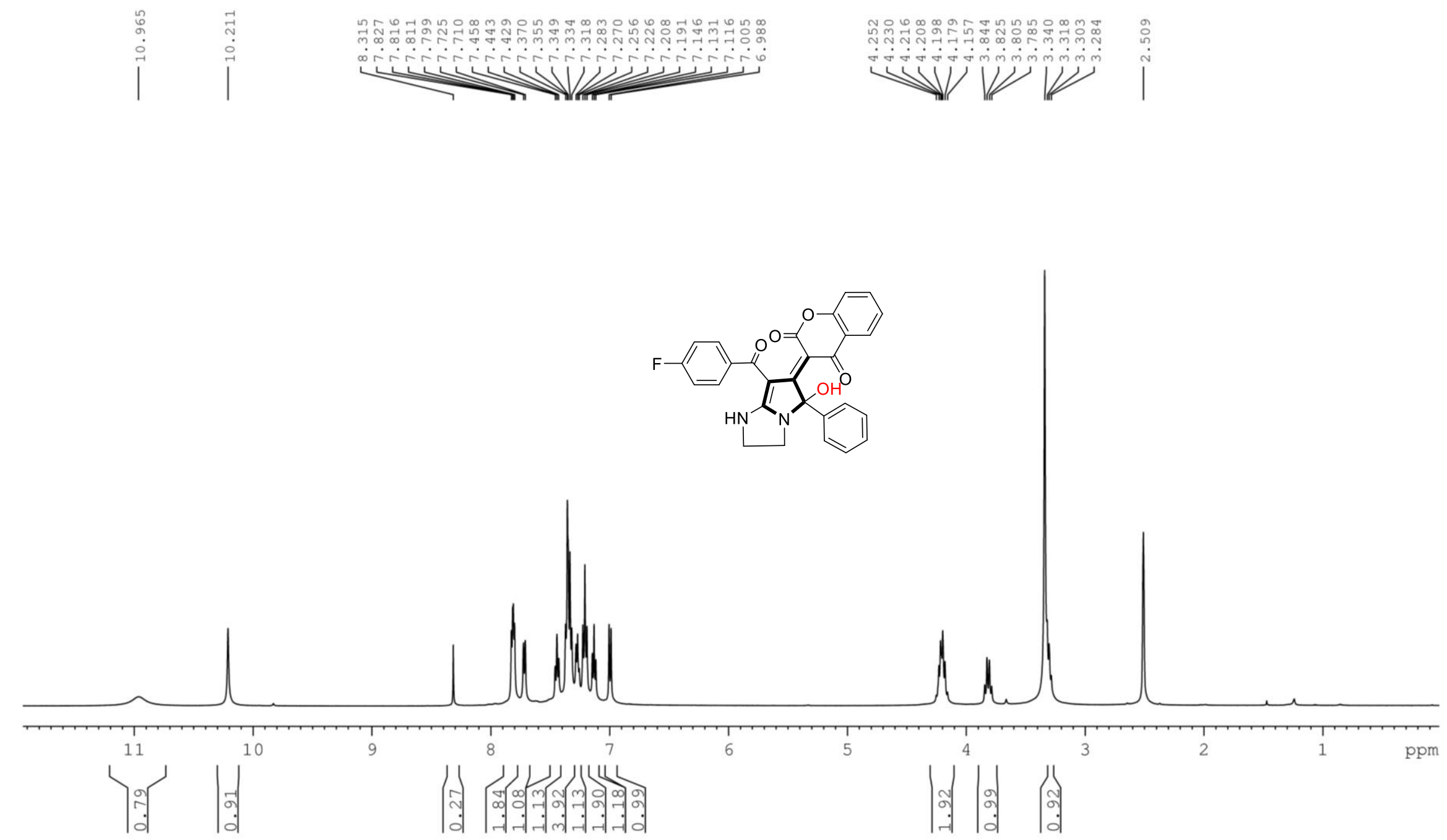

Figure S53. ${ }^{1} \mathrm{H}$ NMR $\left(500 \mathrm{MHz}, \mathrm{DMSO}-d_{6}\right)$ spectra of compound $\mathbf{6 d}$ 


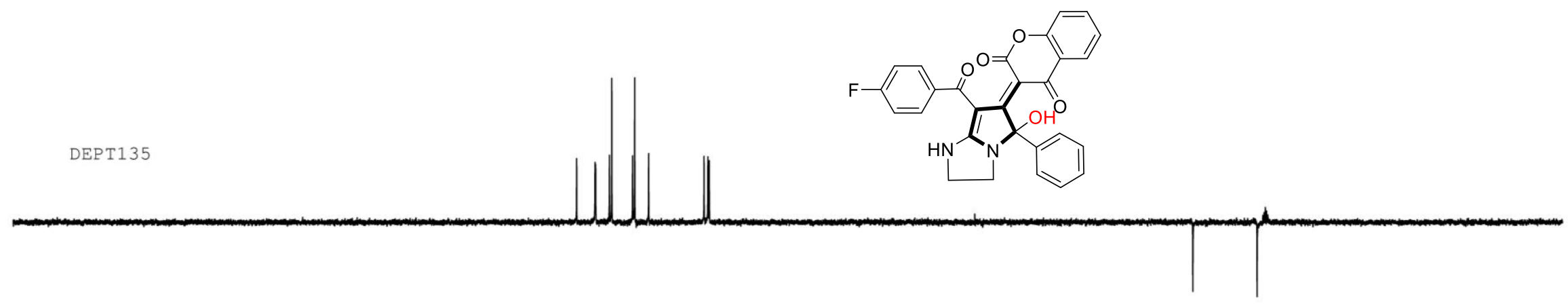

YUNNAN UNIVERSITY AVIIIHD500 ZQX-a-7 Jul06-2018-ziquanxing

C13CPD DMSO

$$
\text { | }
$$

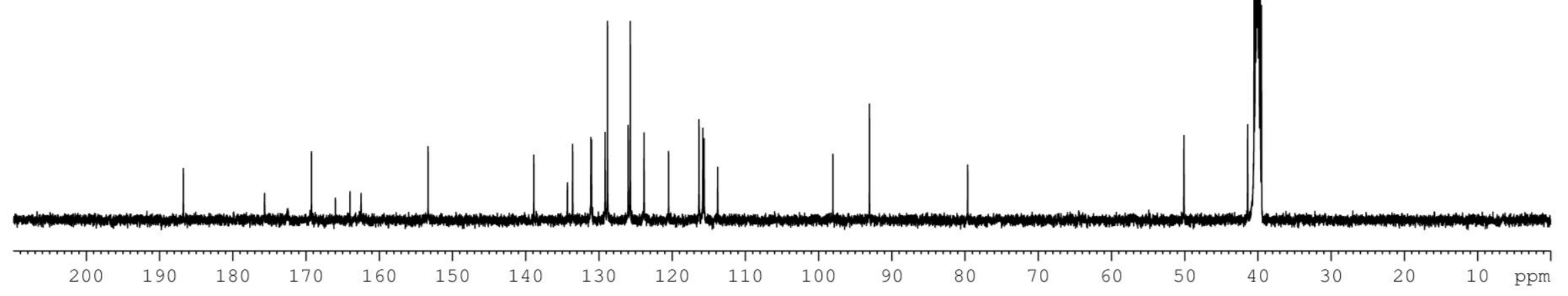

Figure S54. ${ }^{13} \mathrm{C}\left\{{ }^{1} \mathrm{H}\right\}$ NMR $\left(125 \mathrm{MHz}, \mathrm{DMSO}-d_{6}\right)$ spectra of compound $\mathbf{6 d}$ 


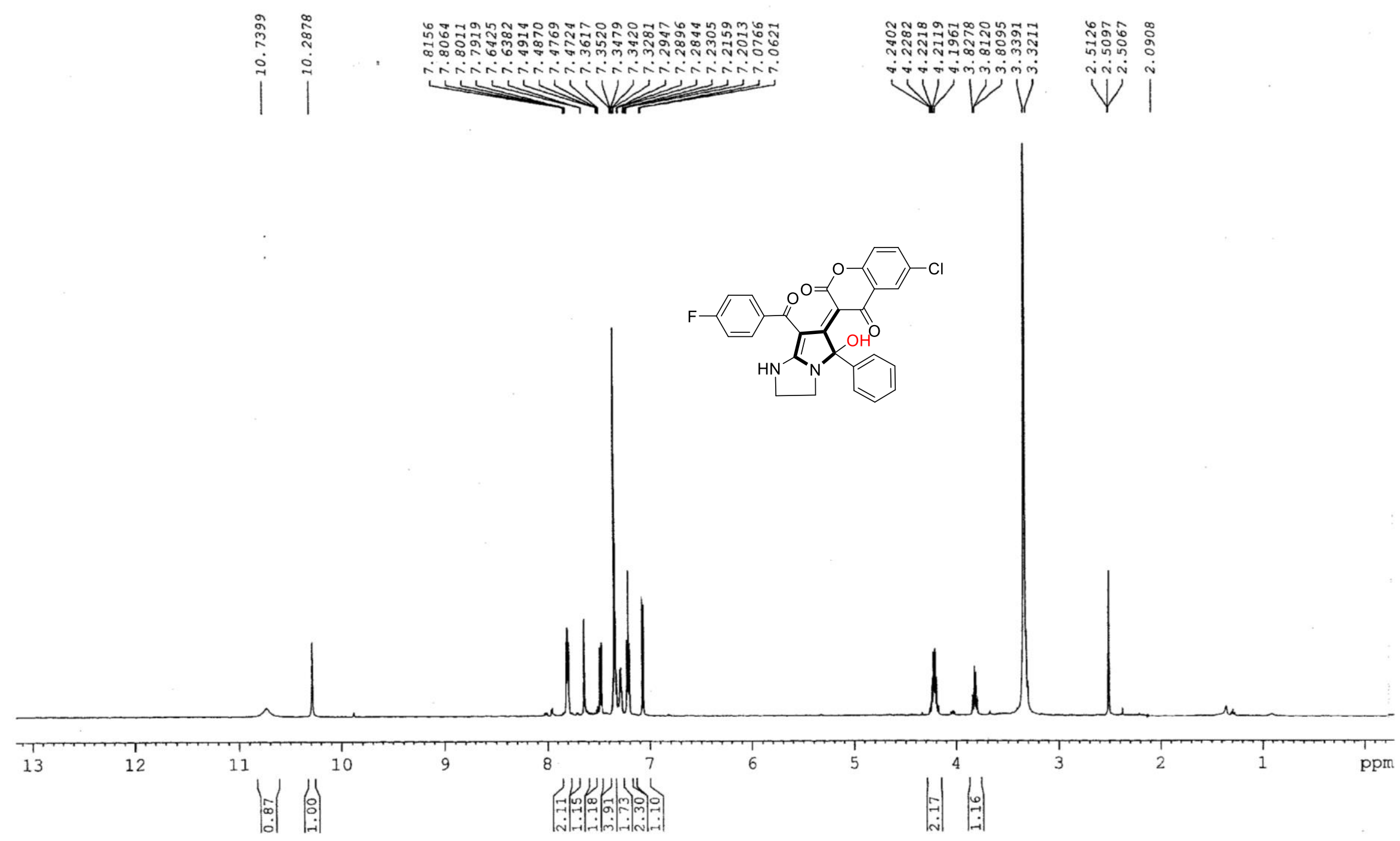

Figure S55. ${ }^{1} \mathrm{H}$ NMR $\left(600 \mathrm{MHz}, \mathrm{DMSO}-d_{6}\right)$ spectra of compound $6 \mathbf{e}$ 


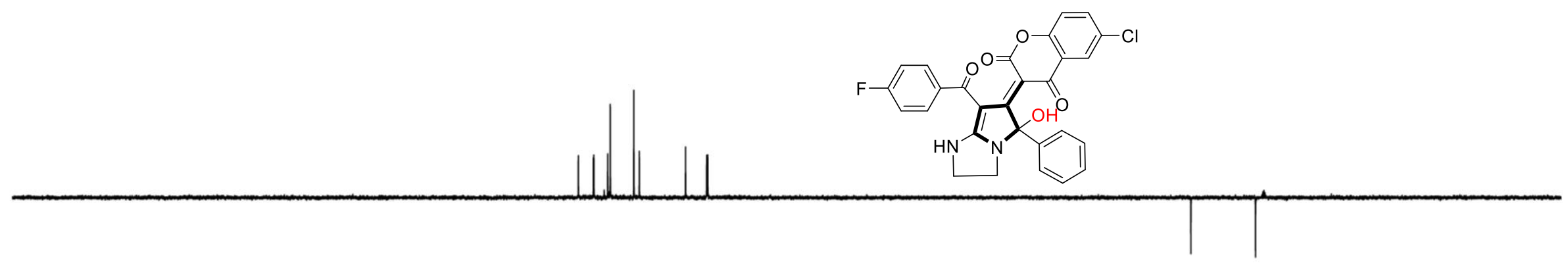

YUNNAN UNIVERSITY ASCEND AVIIIHD600 ZQX-a-29-1

Aug10-2018-ziquanxing

C13CPD DMSO

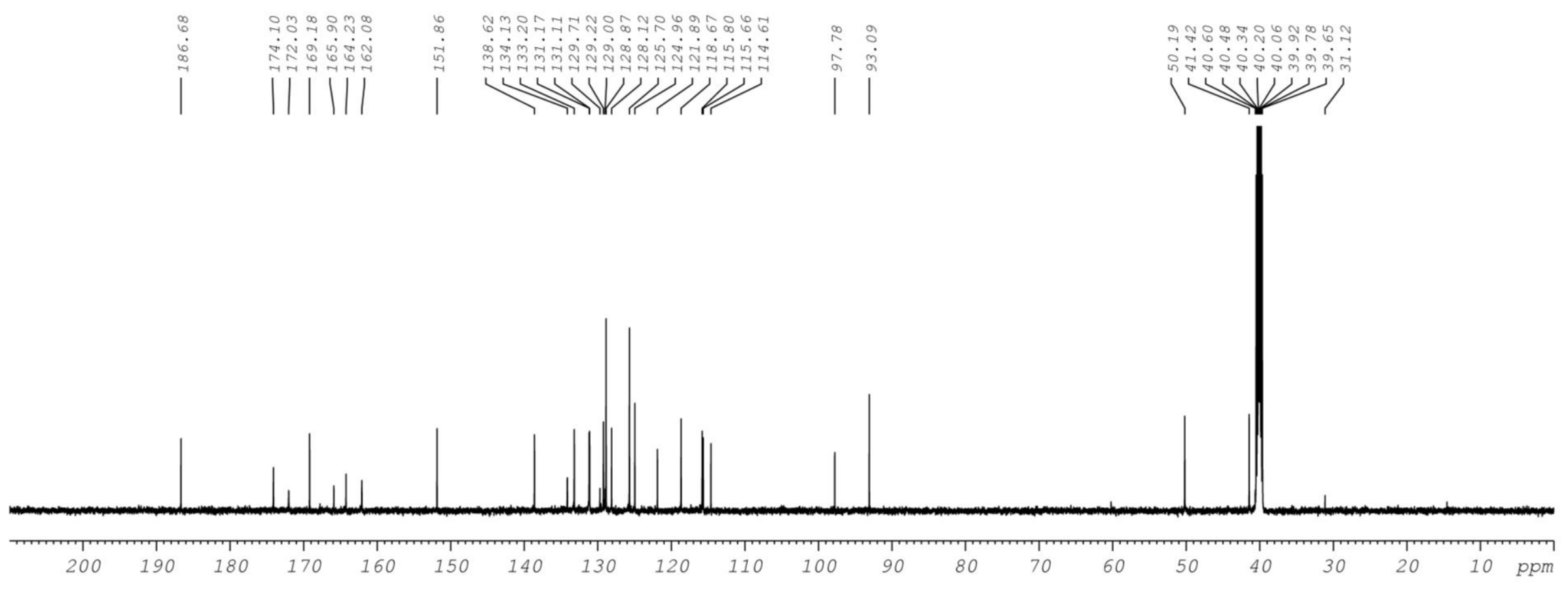

Figure S56. ${ }^{13} \mathrm{C}\left\{{ }^{1} \mathrm{H}\right\}$ NMR (150 MHz, DMSO- $\left.d_{6}\right)$ spectra of compound $6 \mathbf{e}$ 


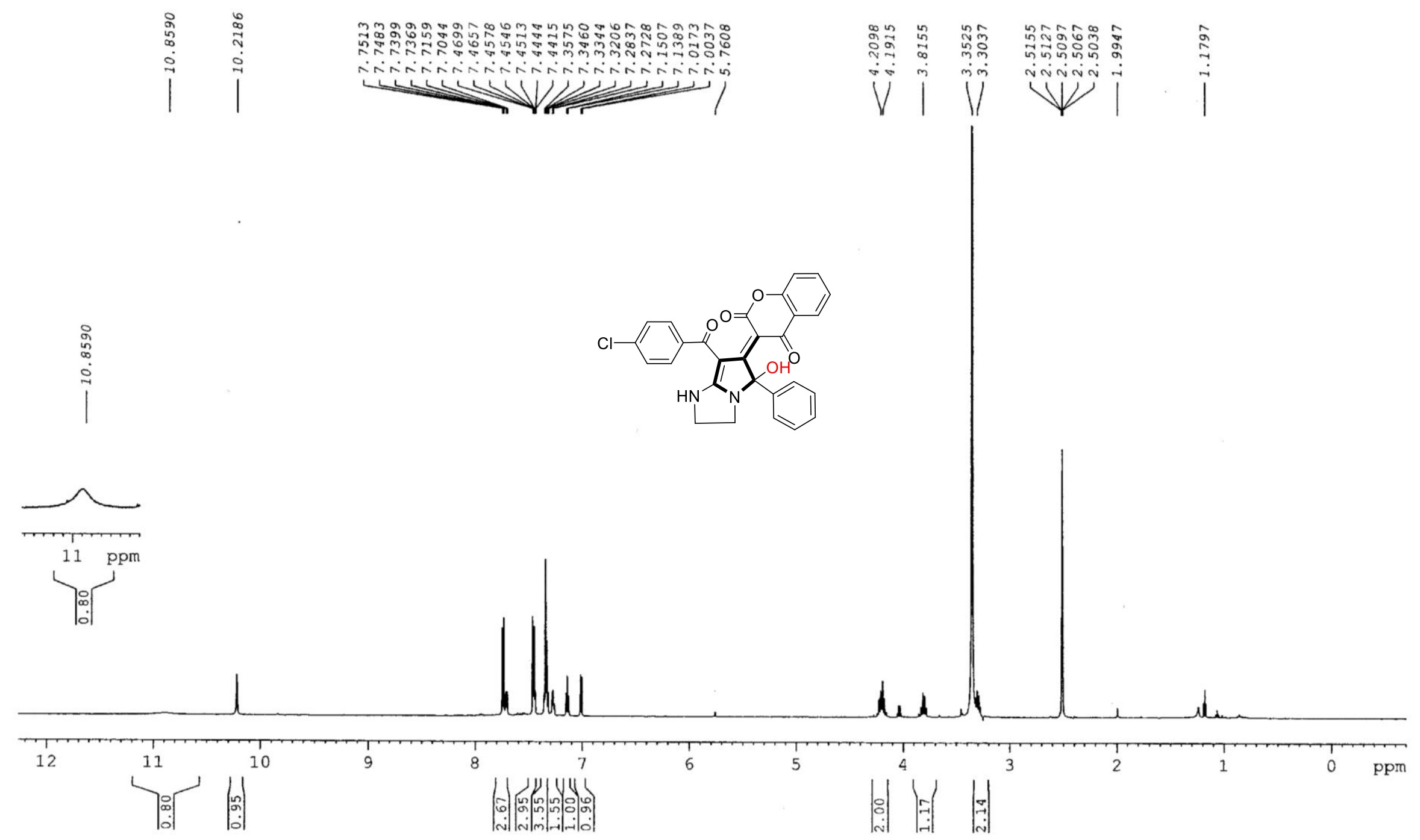

Figure S57. ${ }^{1} \mathrm{H}$ NMR $\left(600 \mathrm{MHz}, \mathrm{DMSO}-d_{6}\right)$ spectra of compound $\mathbf{6 f}$ 


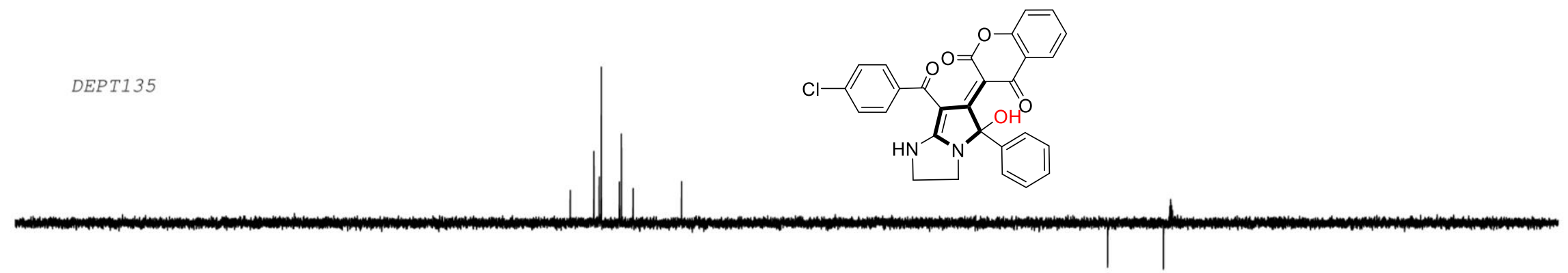

YUNNAN UNIVERSITY ASCEND AVIIIHD600 ZQX-A-36 Oct 26-2018-ziquanxing

C13CPD DMSO

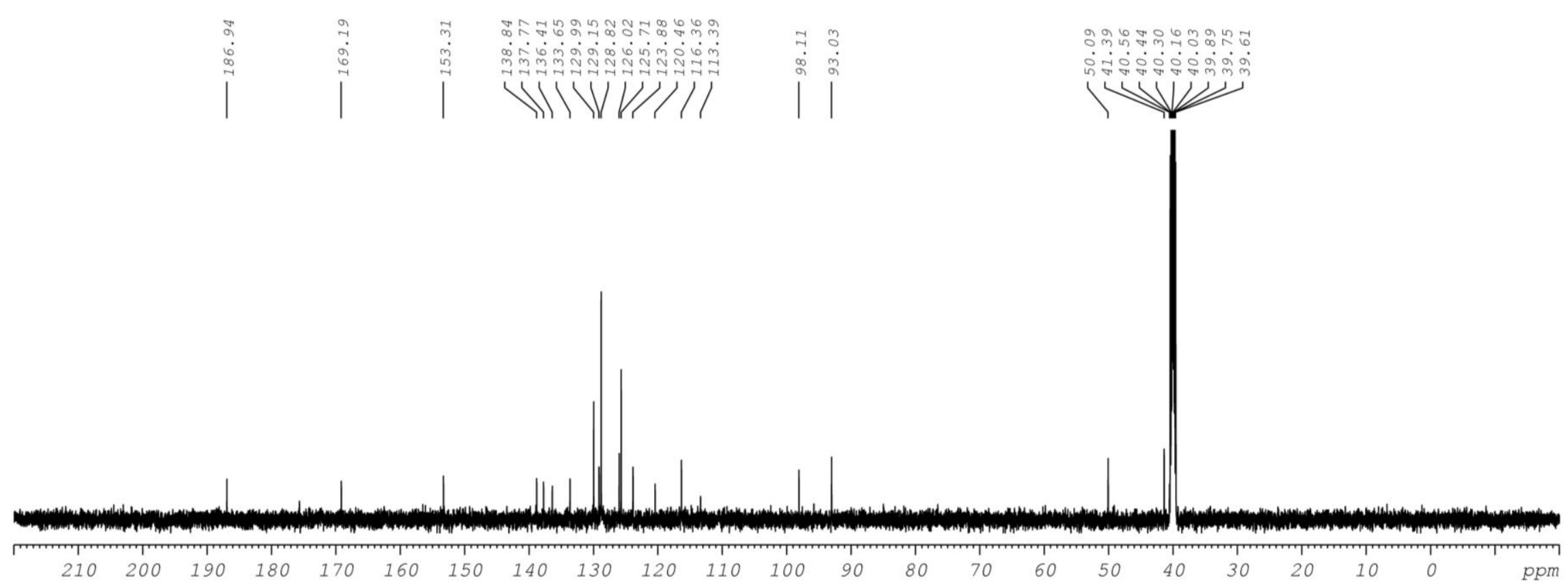

Figure S58. ${ }^{13} \mathrm{C}\left\{{ }^{1} \mathrm{H}\right\}$ NMR $\left(150 \mathrm{MHz}\right.$, DMSO- $\left.d_{6}\right)$ spectra of compound $\mathbf{6 f}$ 


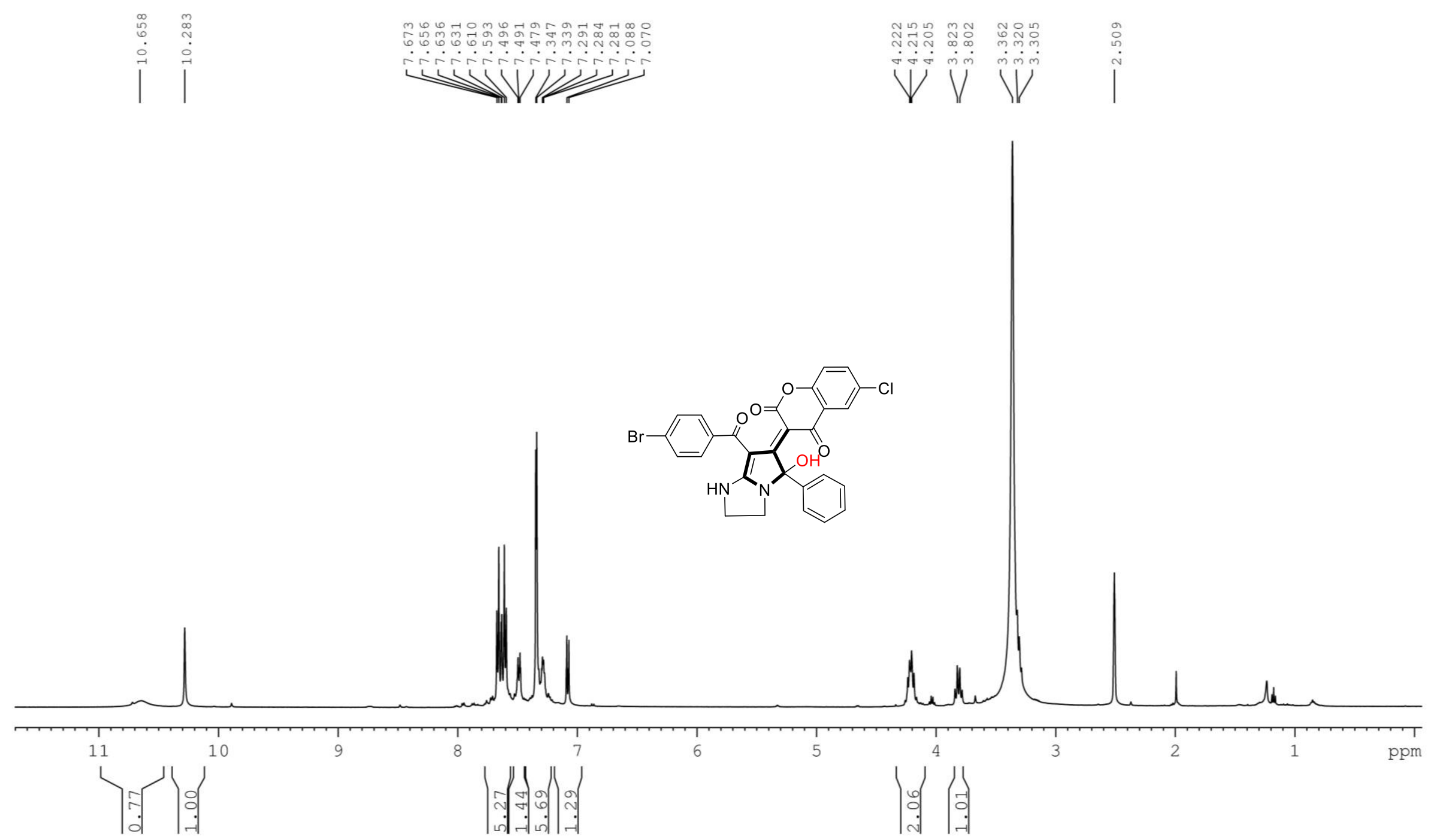

Figure S59. ${ }^{1} \mathrm{H}$ NMR $\left(500 \mathrm{MHz}, \mathrm{DMSO}-d_{6}\right)$ spectra of compound $\mathbf{6 g}$ 


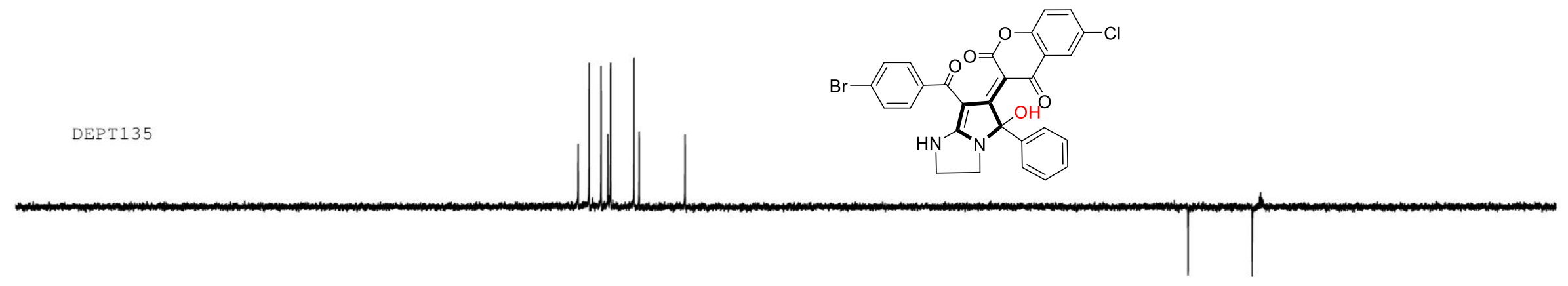

YUNNAN UNIVERSITY AVIIIHD500 ZQX-a-29

Aug09-2018-ziquanxing

C13CPD DMSO

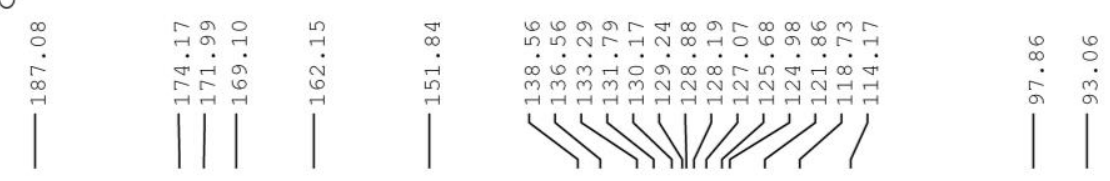

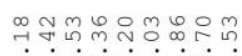

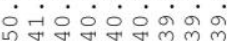

(W)
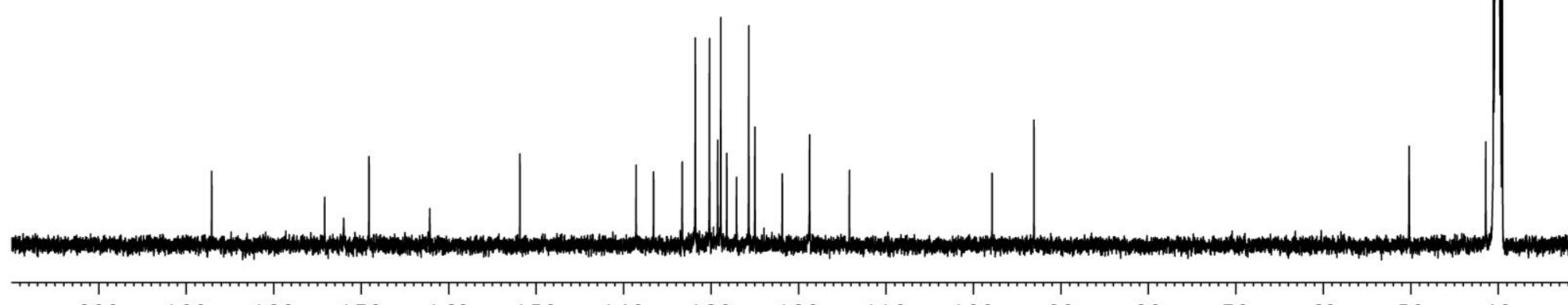

$200 \quad 190 \quad 180$

110100

90

80

Figure S60. ${ }^{13} \mathrm{C}\left\{{ }^{1} \mathrm{H}\right\}$ NMR (125 MHz, DMSO- $\left.d_{6}\right)$ spectra of compound $\mathbf{6 g}$ 


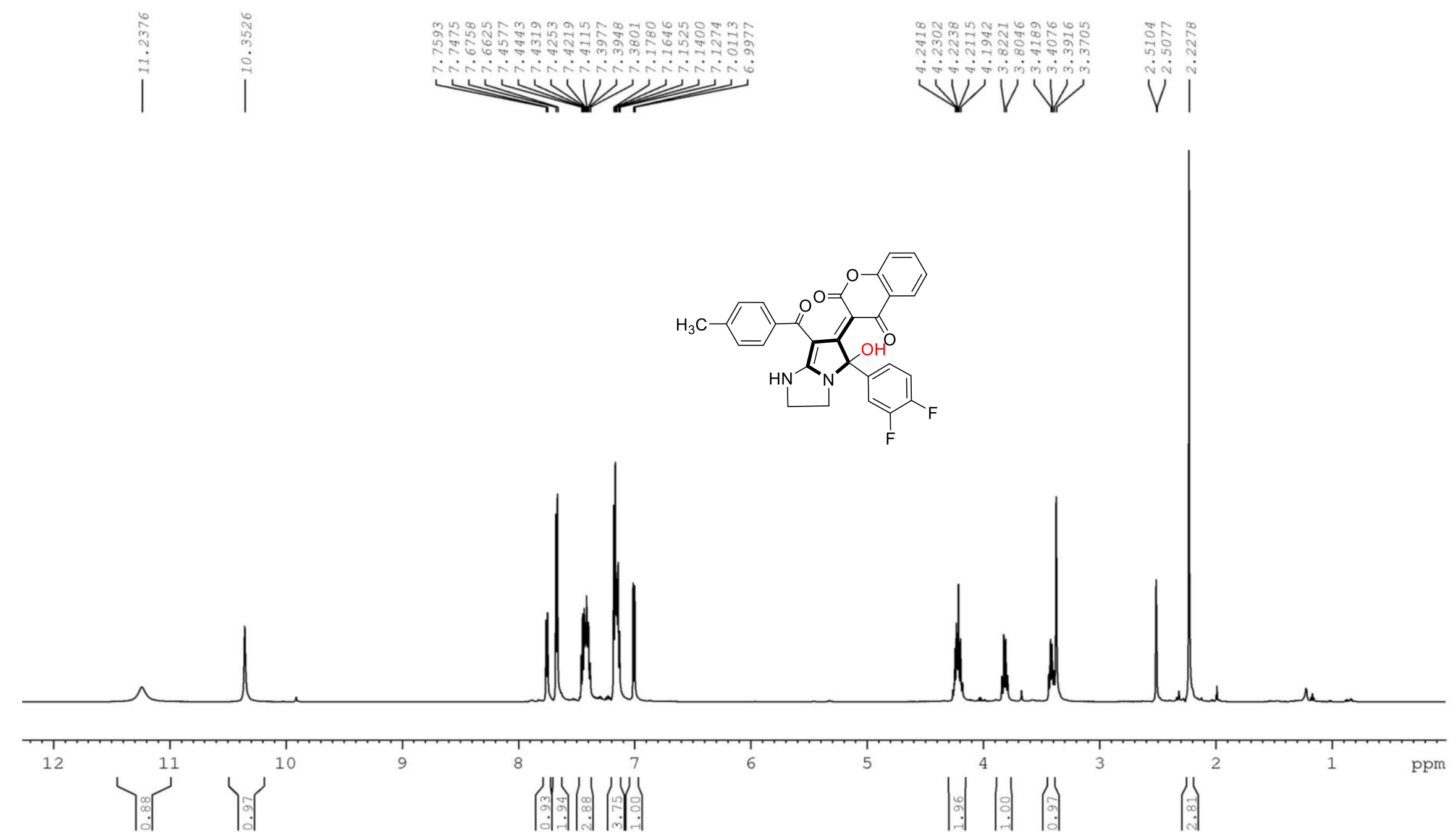

Figure S61. ${ }^{1} \mathrm{H}$ NMR $\left(500 \mathrm{MHz}, \mathrm{DMSO}-d_{6}\right)$ spectra of compound $\mathbf{6 h}$ 


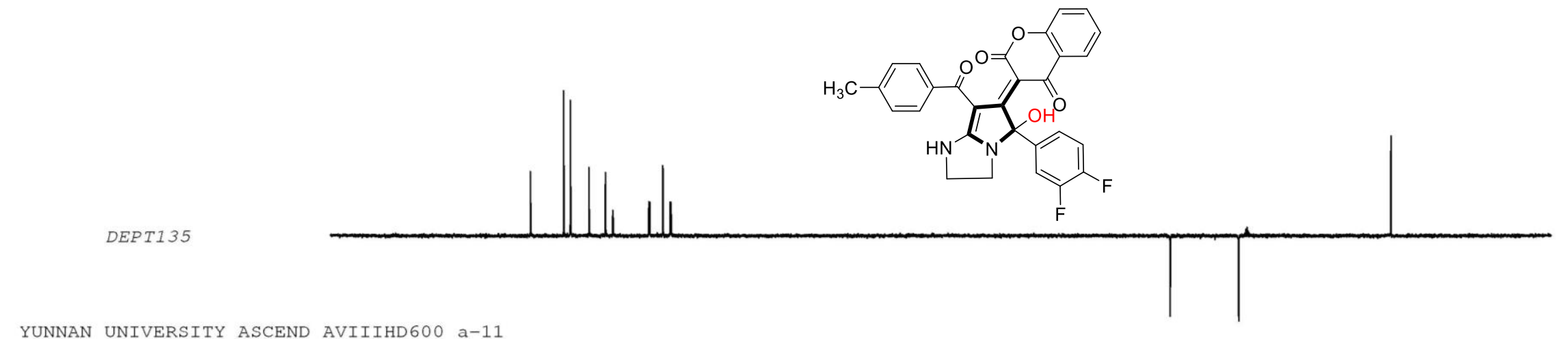

YUNNAN UNIVERSITY ASCEND AVIIIHD600 a-11 C13CPD DMSO

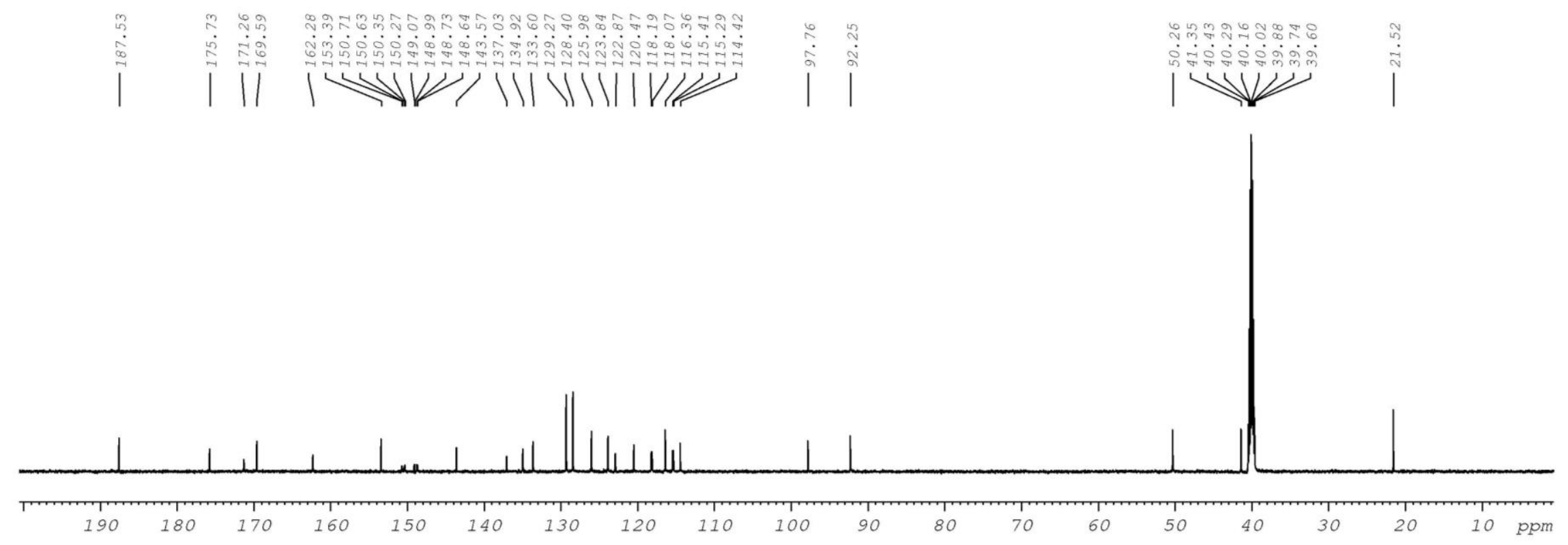

Figure S62. ${ }^{13} \mathrm{C}\left\{{ }^{1} \mathrm{H}\right\}$ NMR $\left(125 \mathrm{MHz}\right.$, DMSO- $\left.d_{6}\right)$ spectra of compound $\mathbf{6 h}$ 

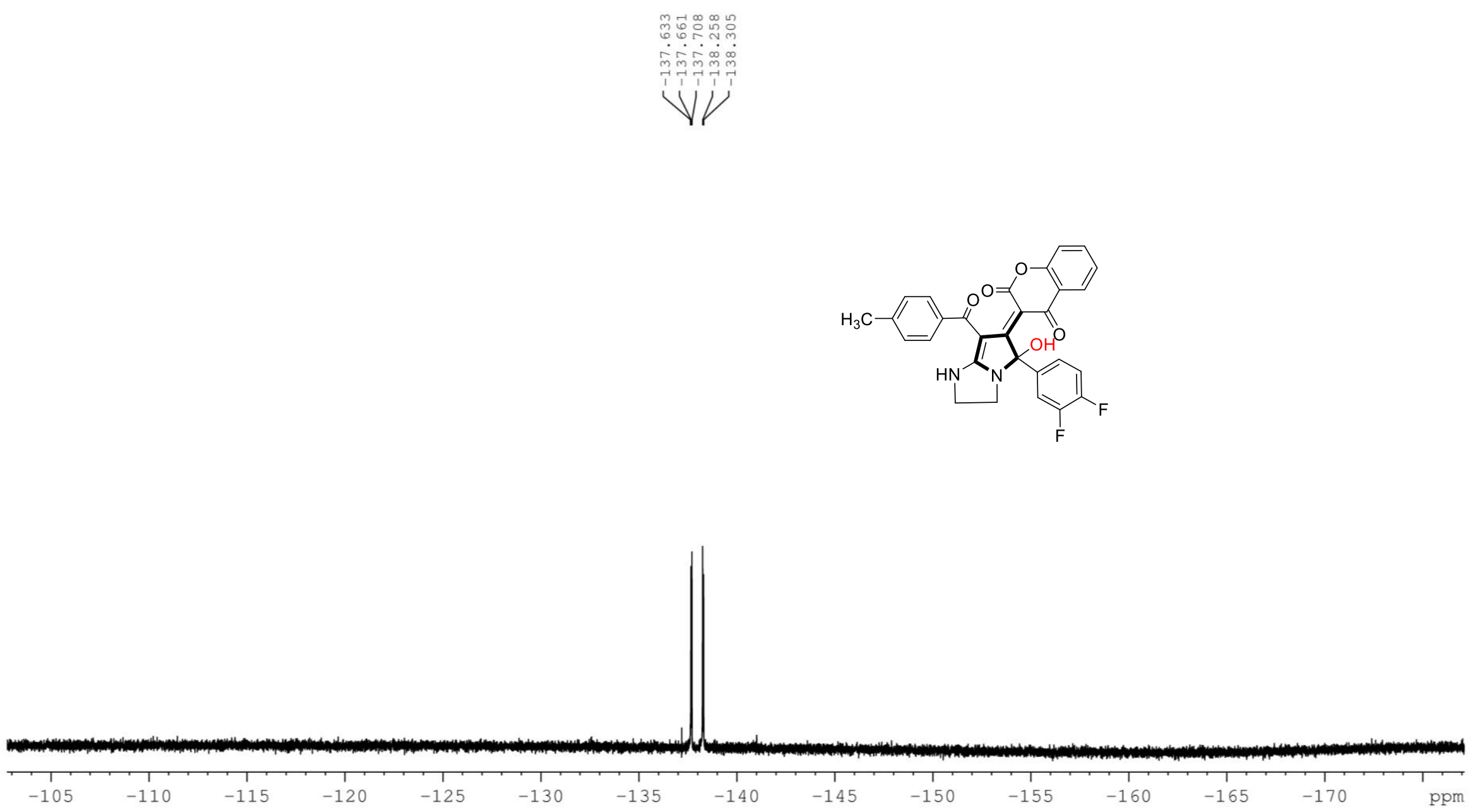

Figure S63. ${ }^{19}$ F NMR $\left(470 \mathrm{MHz}\right.$, DMSO- $\left.d_{6}\right)$ spectra of compound $\mathbf{6 h}$ 

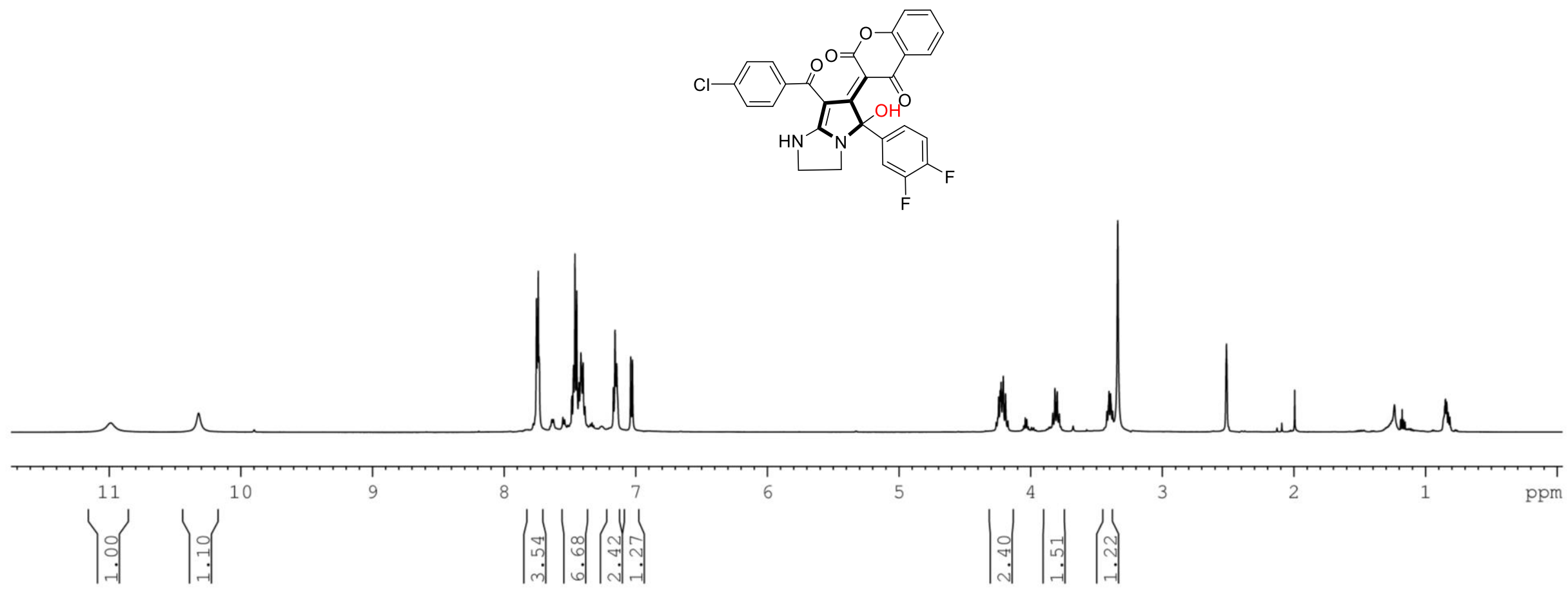

Figure S64. ${ }^{1} \mathrm{H}$ NMR $\left(600 \mathrm{MHz}, \mathrm{DMSO}-d_{6}\right)$ spectra of compound $\mathbf{6 i}$ 


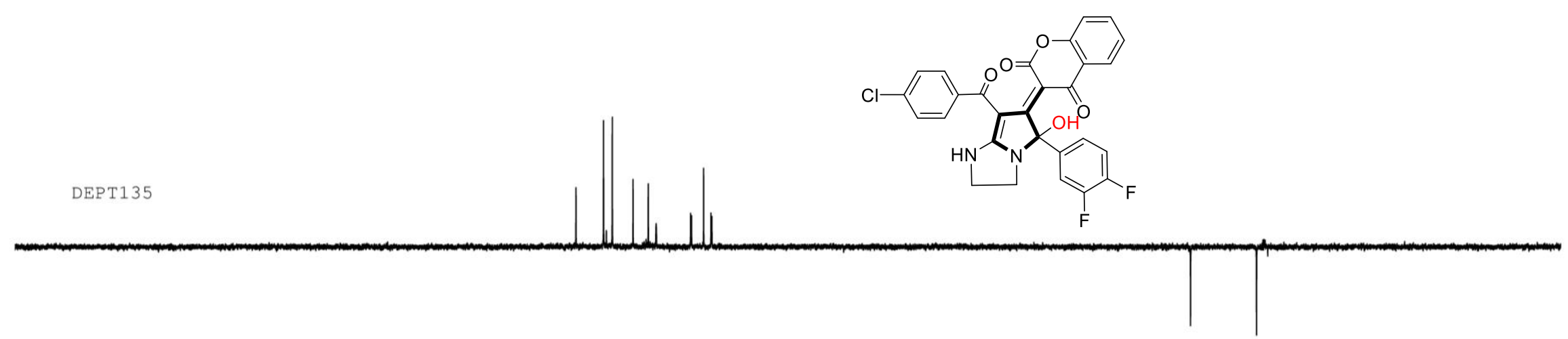

YUNNAN UNIVERSITY ASCEND AVIIIHD600 ZQX-A-20 Aug27-2018-ziquanxing

C13CPD DMSO

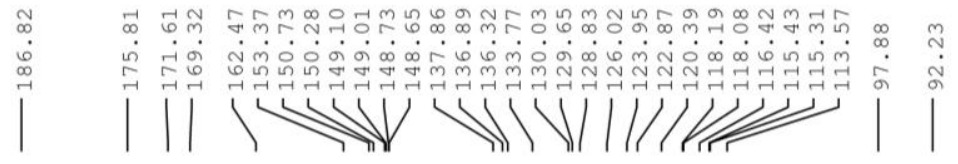

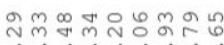

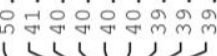

(N)
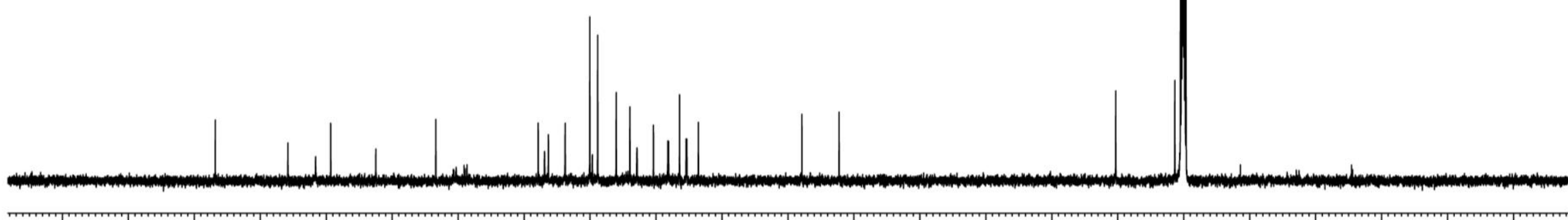

$210-200-190-180-170-160-150-140-130=120101000$

50

Figure S65. ${ }^{13} \mathrm{C}\left\{{ }^{1} \mathrm{H}\right\}$ NMR (150 MHz, DMSO-d $)$ spectra of compound $6 \mathbf{i}$ 


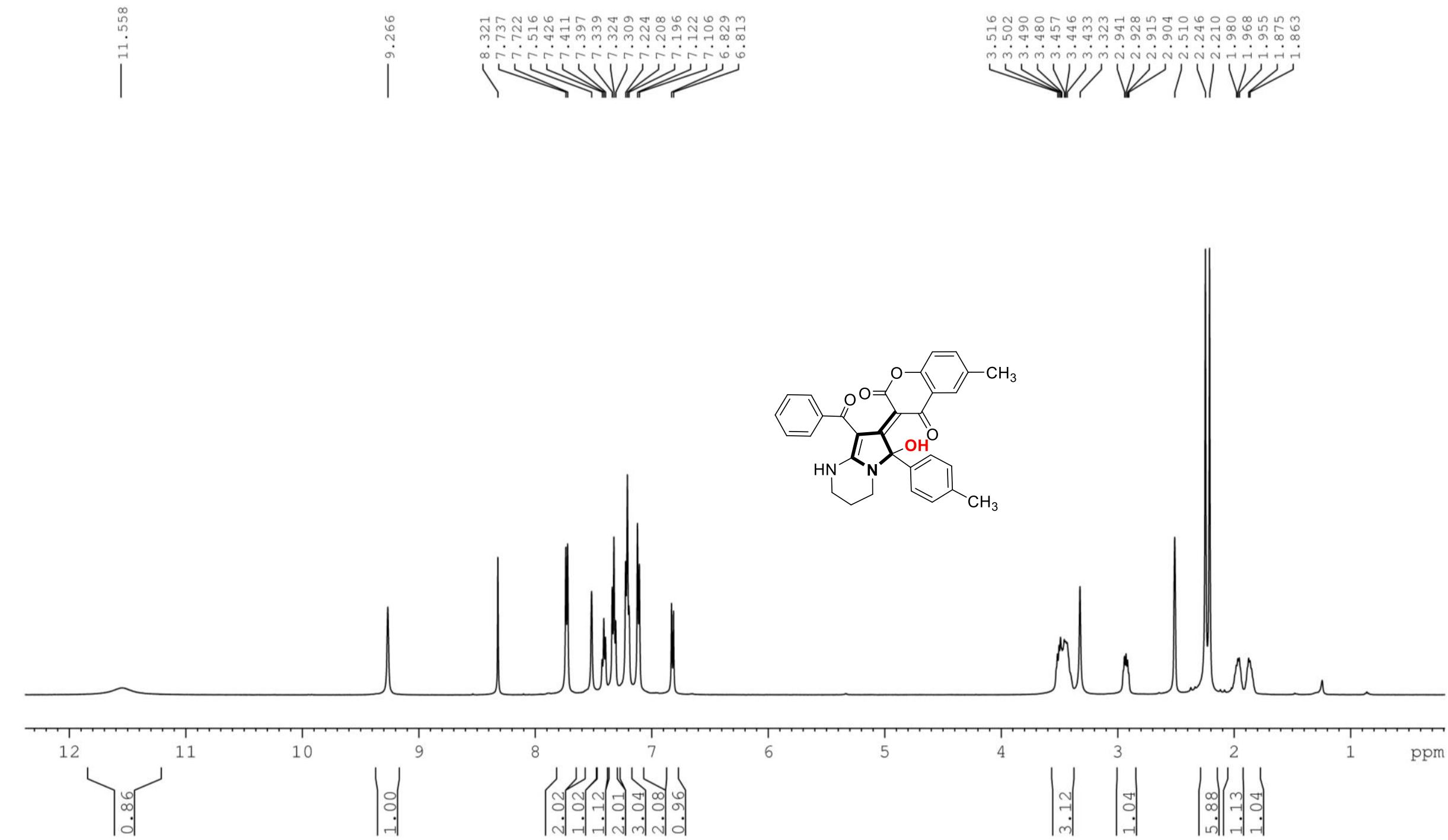

Figure S66. ${ }^{1} \mathrm{H}$ NMR $\left(500 \mathrm{MHz}, \mathrm{DMSO}-d_{6}\right)$ spectra of compound $\mathbf{6 j}$ 


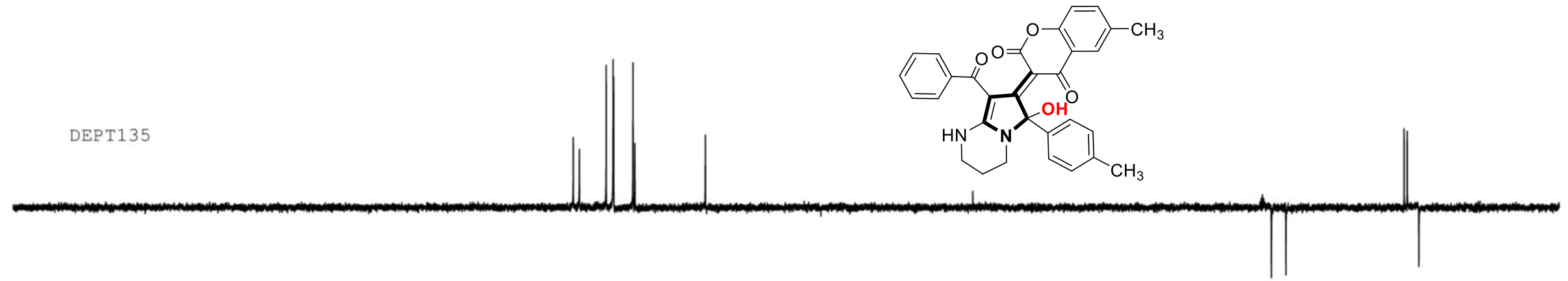

YUNNAN UNIVERSITY AVIIIHD500 ZOX-a-12 Jul18-2018-ziquanxing C13CPD DMSO
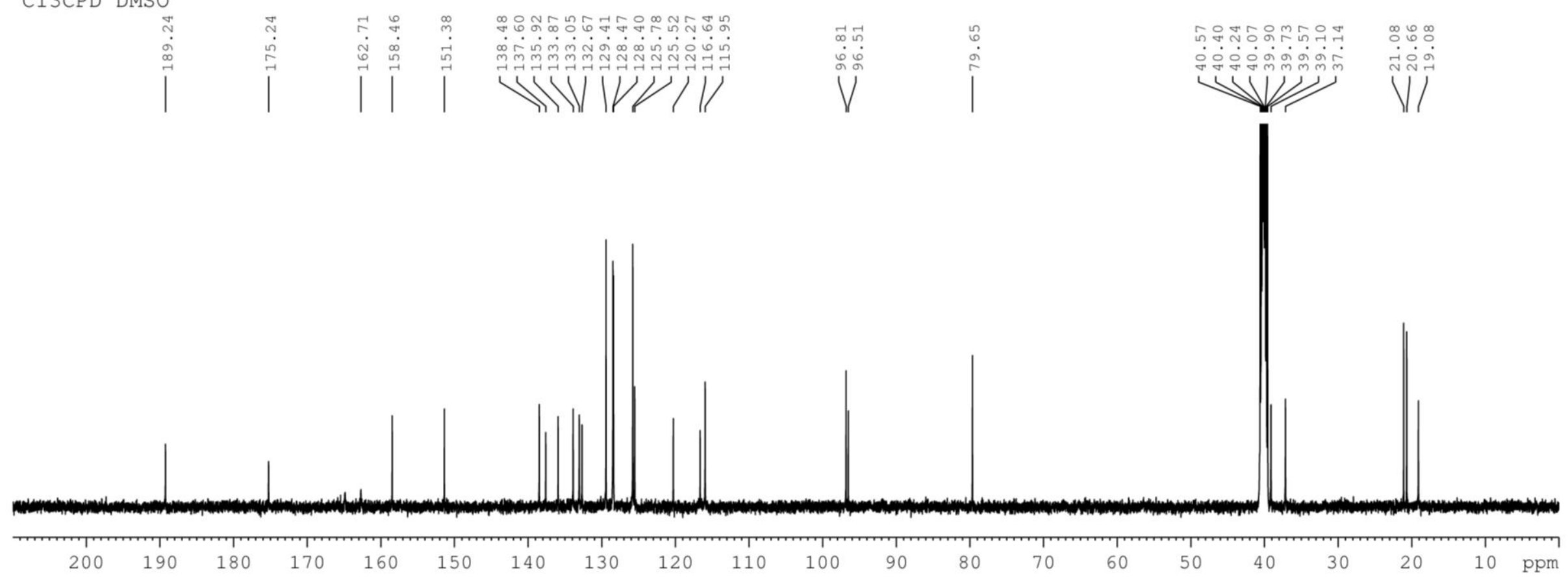

Figure S67. ${ }^{13} \mathrm{C}\left\{{ }^{1} \mathrm{H}\right\}$ NMR $\left(125 \mathrm{MHz}, \mathrm{DMSO}-d_{6}\right)$ spectra of compound $\mathbf{6 j}$ 


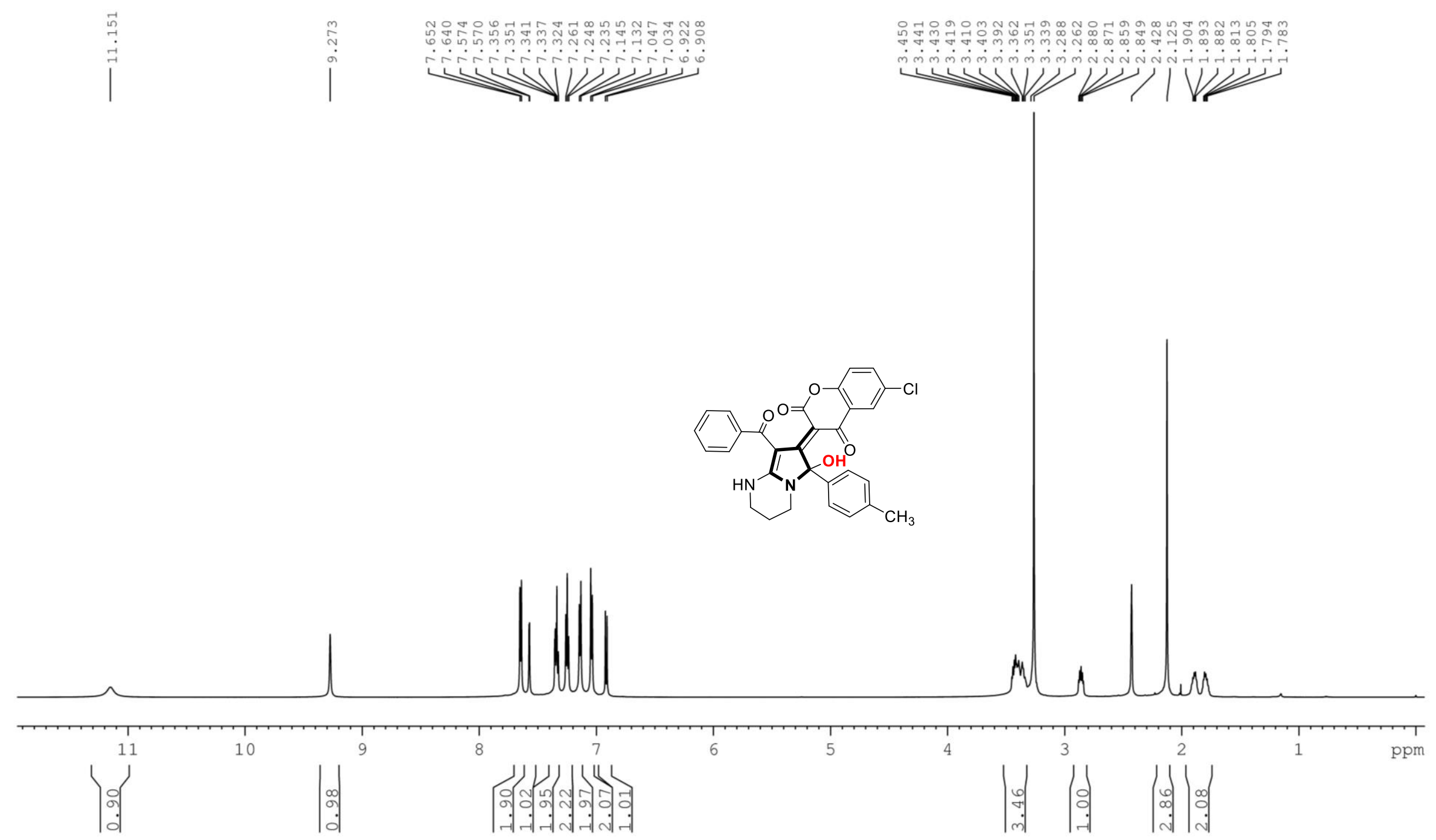

Figure S68. ${ }^{1} \mathrm{H}$ NMR $\left(600 \mathrm{MHz}, \mathrm{DMSO}-d_{6}\right)$ spectra of compound $\mathbf{6 k}$ 


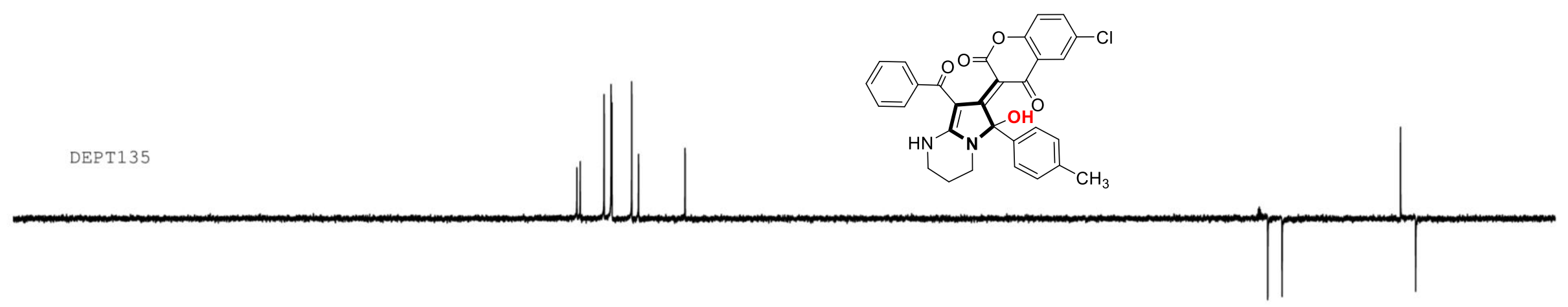

YUNNAN UNIVERSITY ASCEND AVIIIHD600 ZQX-a-23 Aug28-2018-ziquanxing

C13CPD DMSO
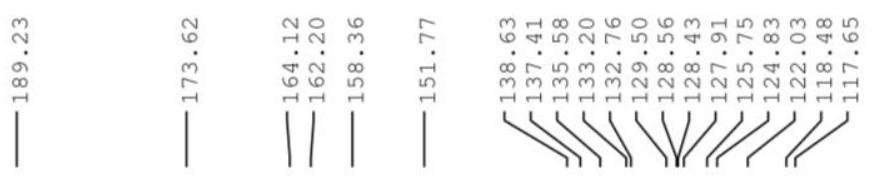

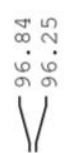
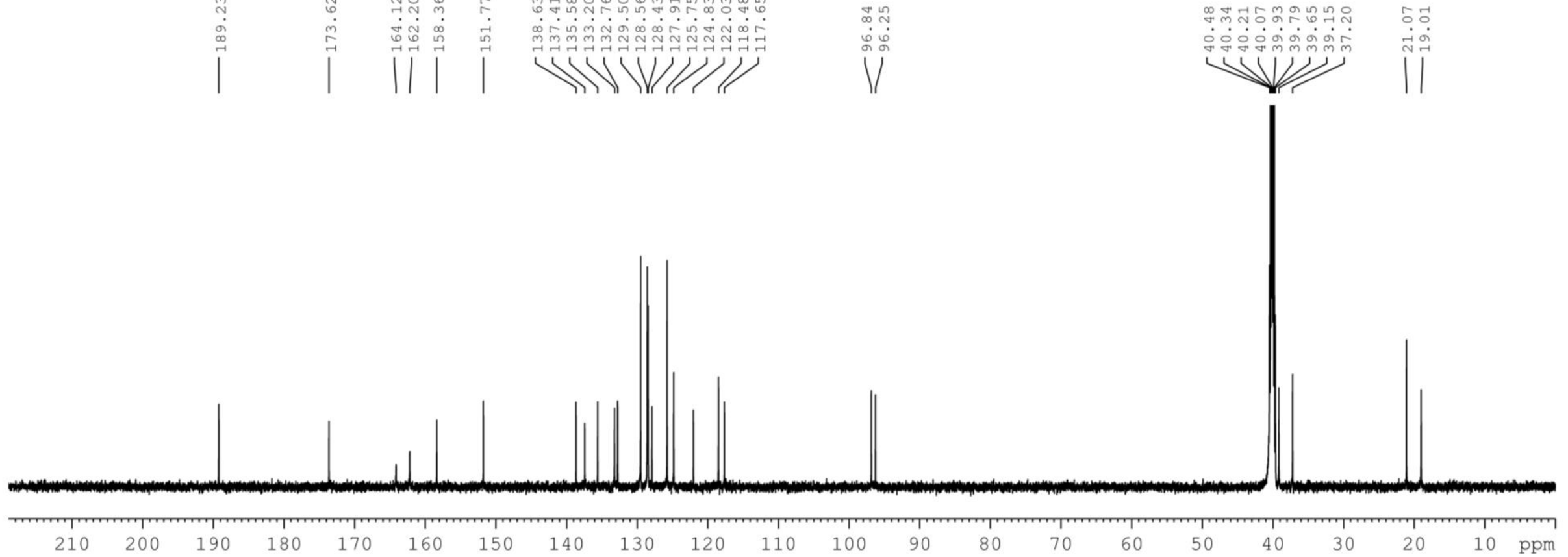

Figure S69. ${ }^{13} \mathrm{C}\left\{{ }^{1} \mathrm{H}\right\}$ NMR (150 MHz, DMSO- $\left.d_{6}\right)$ spectra of compound $\mathbf{6 k}$ 


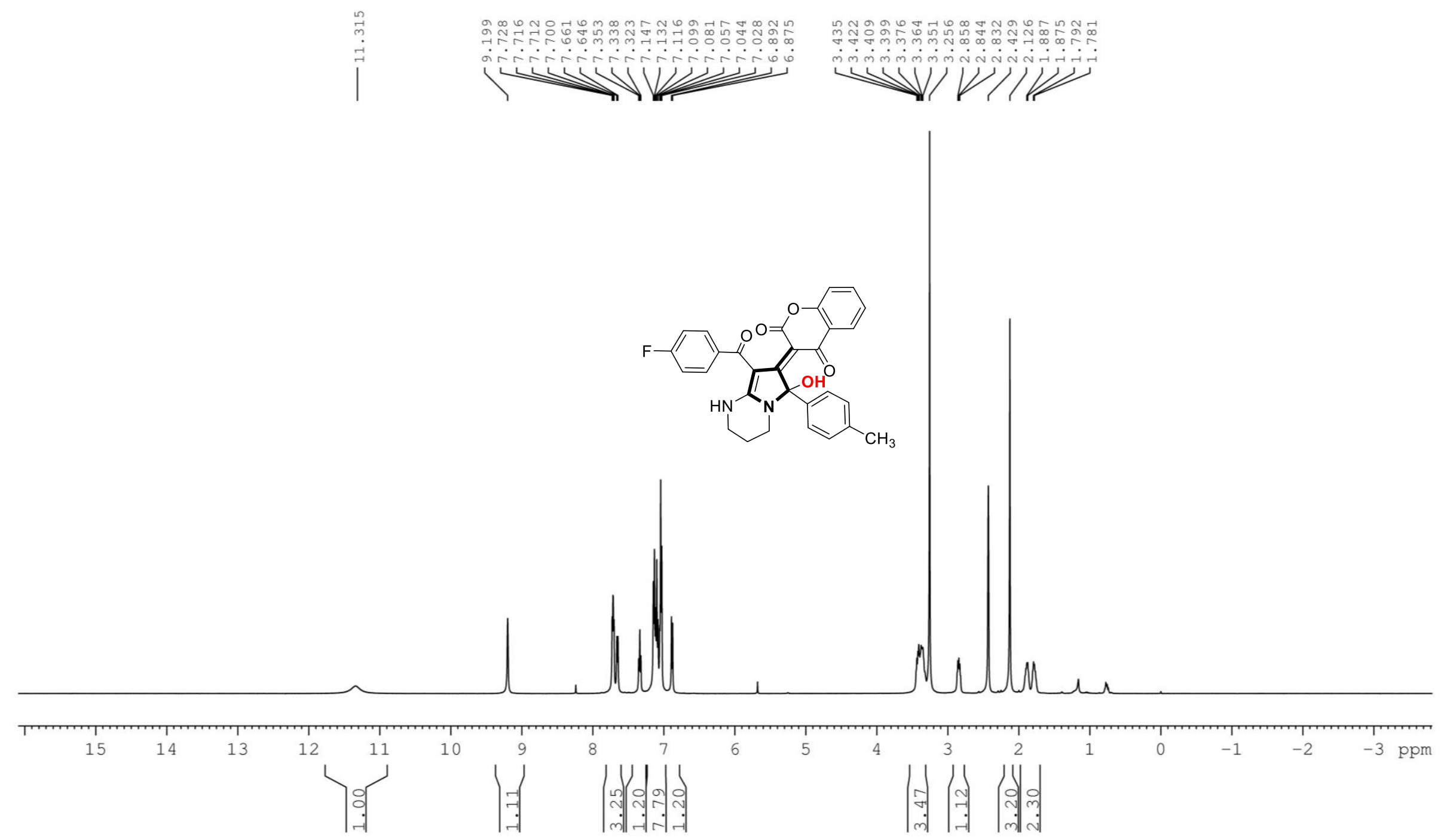

Figure S70. ${ }^{1} \mathrm{H}$ NMR $\left(500 \mathrm{MHz}, \mathrm{DMSO}-d_{6}\right)$ spectra of compound $\mathbf{6}$ 


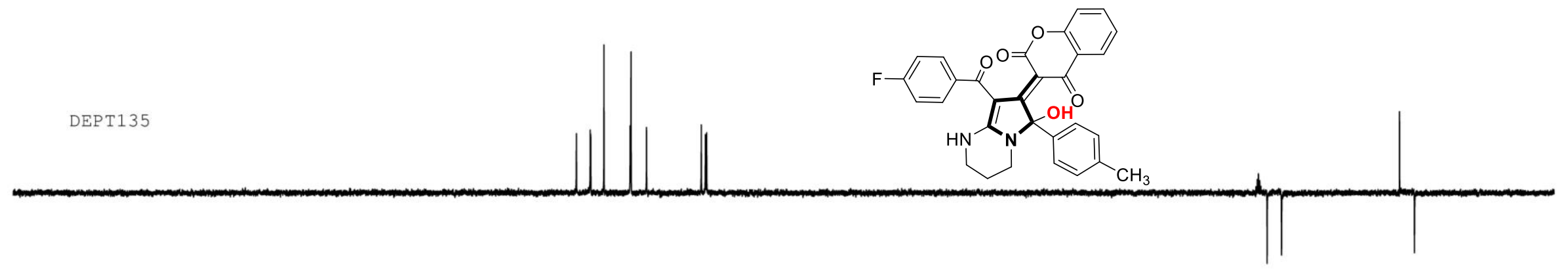

YUNNAN UNIVERSITY AVIIIHD500 ZQX-a-5 Jun27-2018-ziquanxing C13CPD DMSO
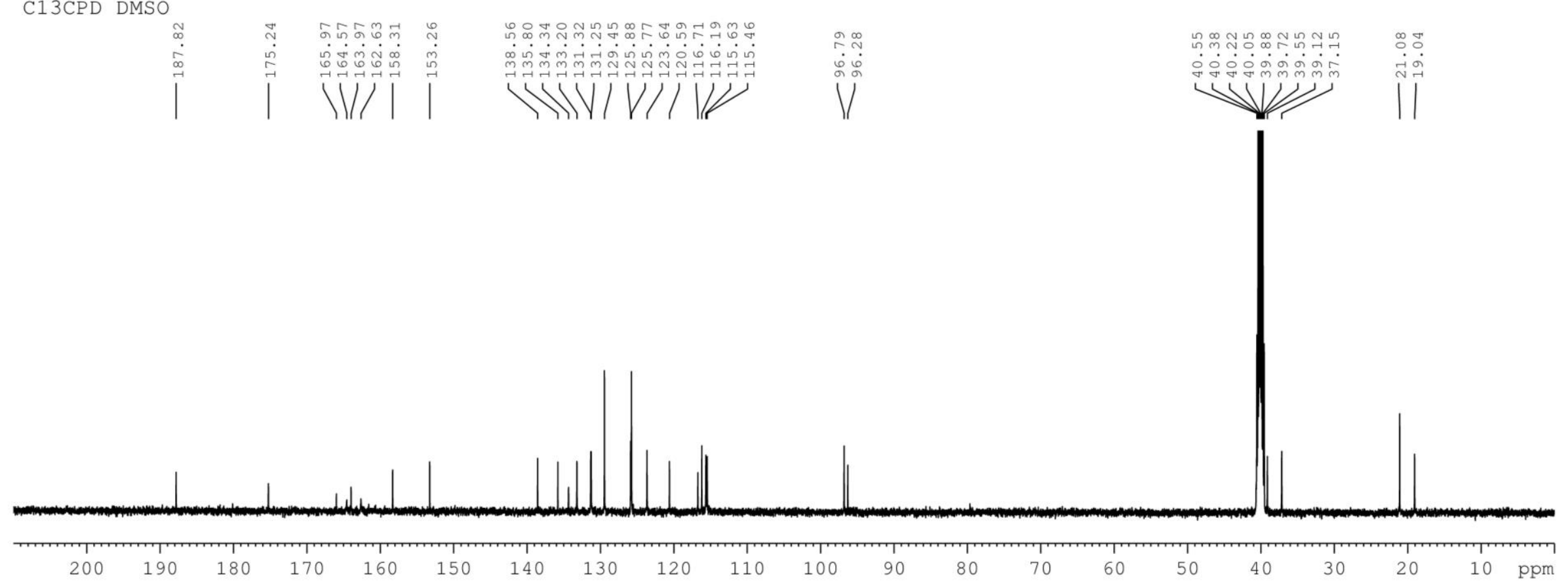

Figure S71. ${ }^{13} \mathrm{C}\left\{{ }^{1} \mathrm{H}\right\}$ NMR (125 MHz, DMSO- $\left.d_{6}\right)$ spectra of compound $\mathbf{6}$ 


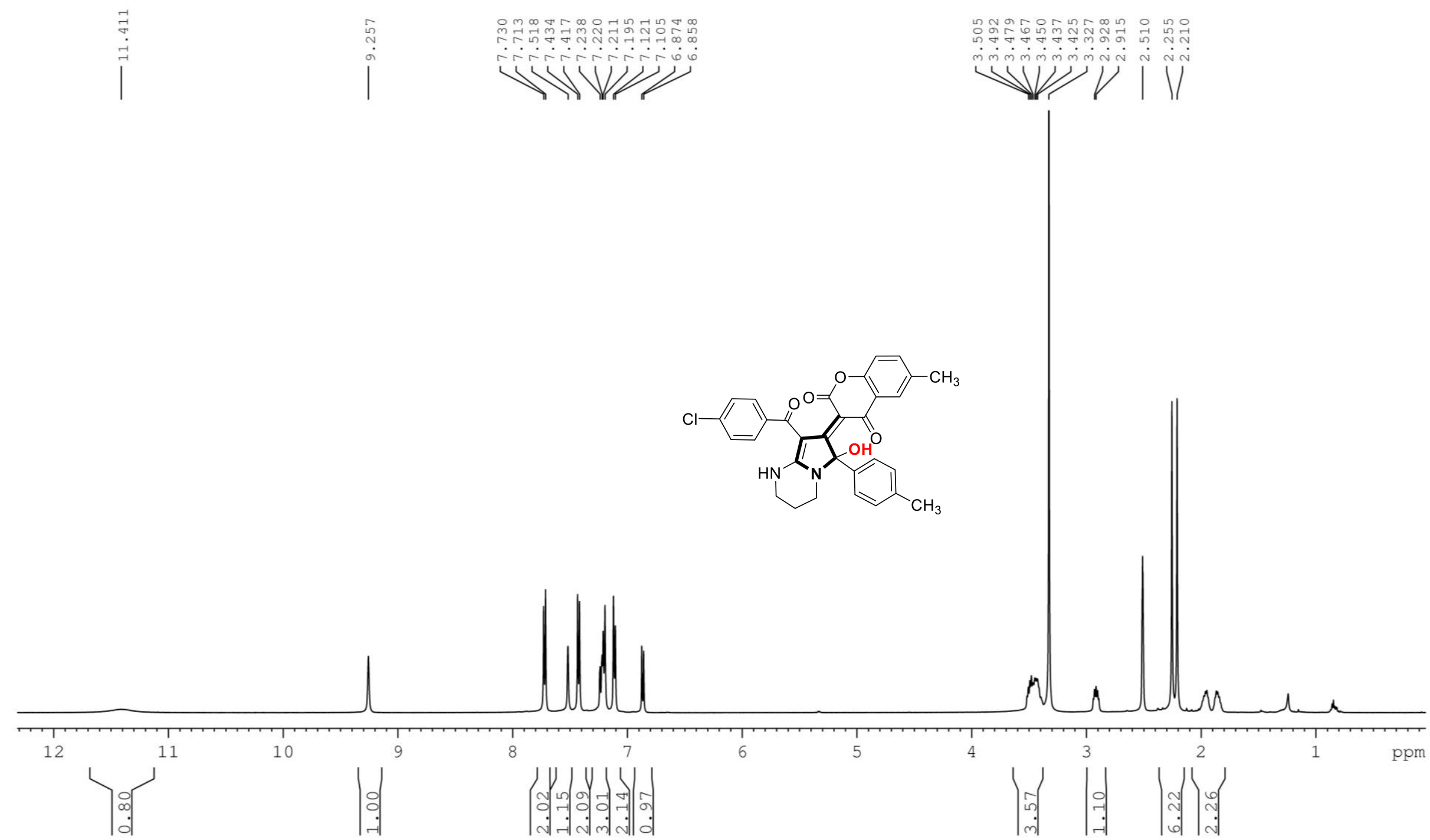

Figure S72. ${ }^{1} \mathrm{H}$ NMR $\left(500 \mathrm{MHz}, \mathrm{DMSO}-d_{6}\right)$ spectra of compound $\mathbf{6 m}$ 


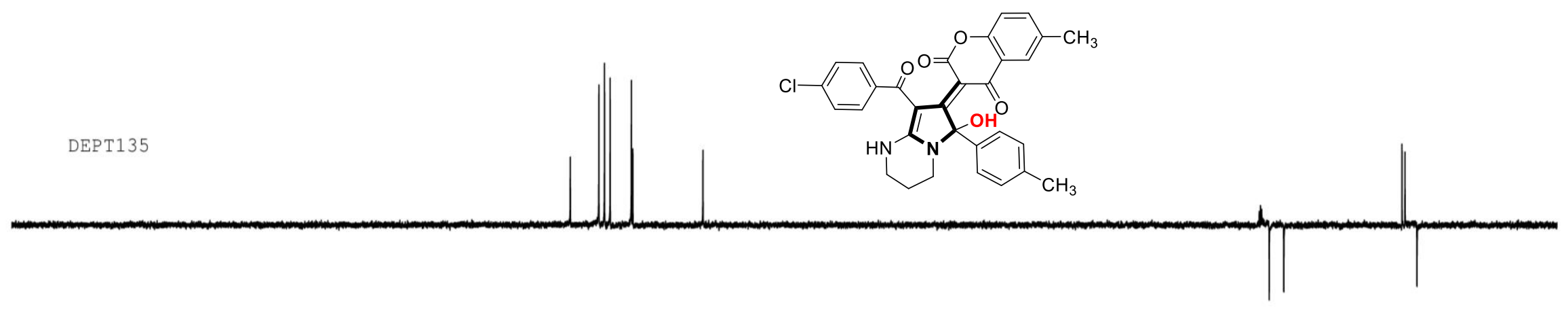

YUNNAN UNIVERSITY AVIIIHD500 ZQX-a-10 Aug02-2018-ziquanxing

C13CPD DMSO
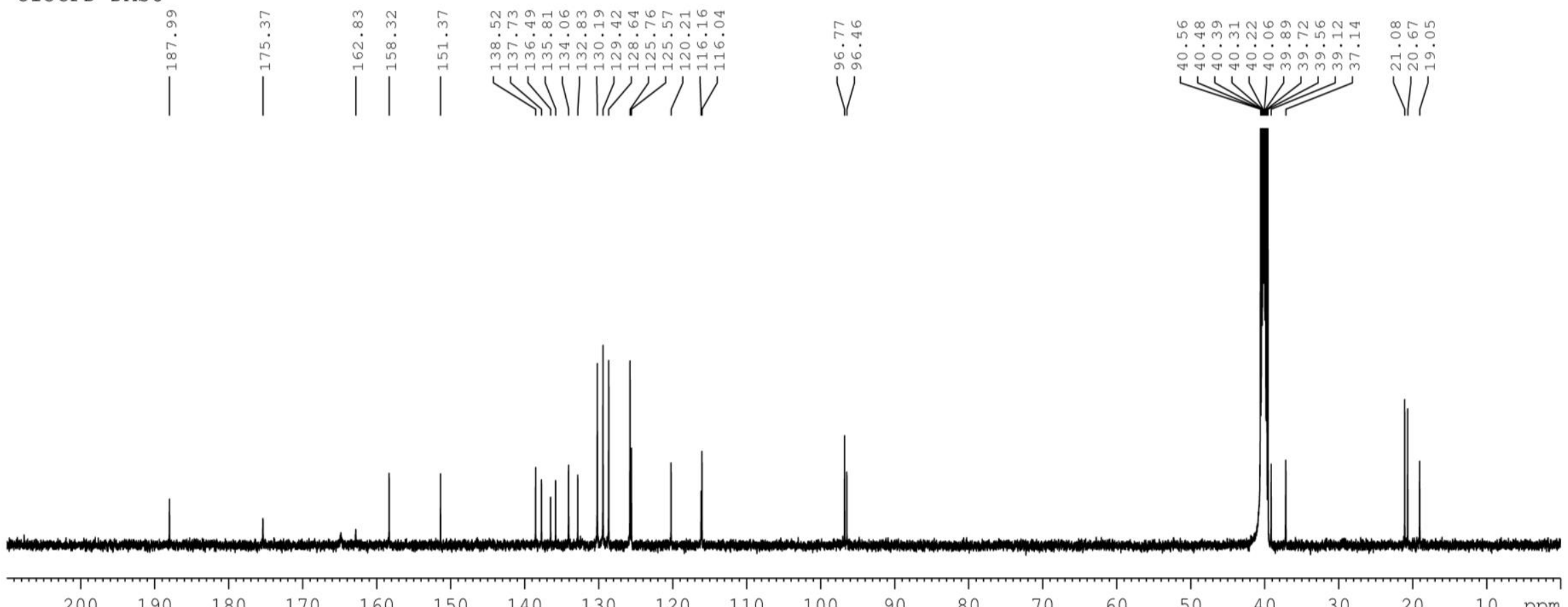

Figure S73. ${ }^{13} \mathrm{C}\left\{{ }^{1} \mathrm{H}\right\}$ NMR $\left(125 \mathrm{MHz}\right.$, DMSO- $\left.d_{6}\right)$ spectra of compound $\mathbf{6 m}$ 


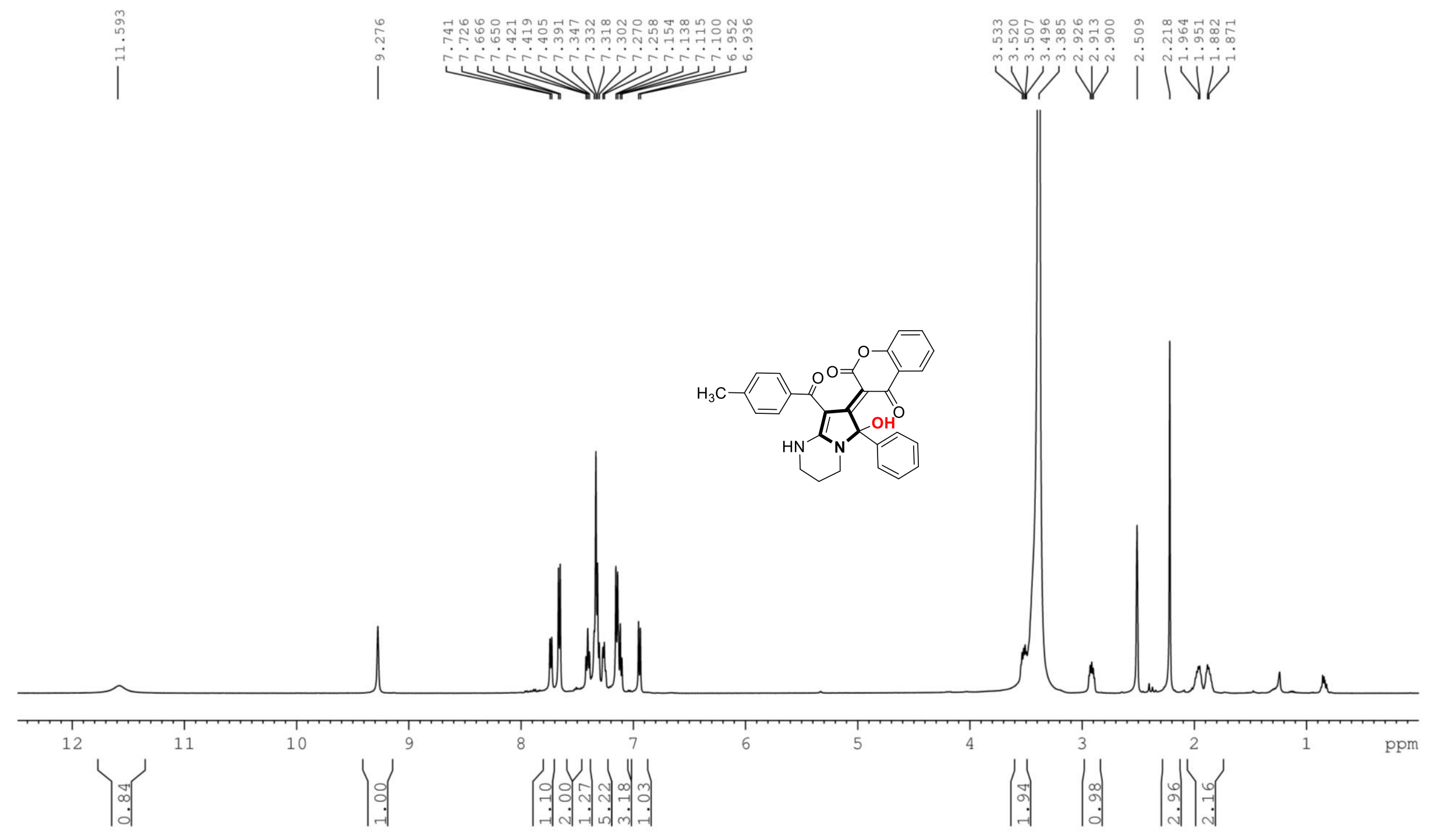

Figure S74. ${ }^{1} \mathrm{H}$ NMR $\left(500 \mathrm{MHz}, \mathrm{DMSO}-d_{6}\right)$ spectra of compound $\mathbf{6 n}$ 


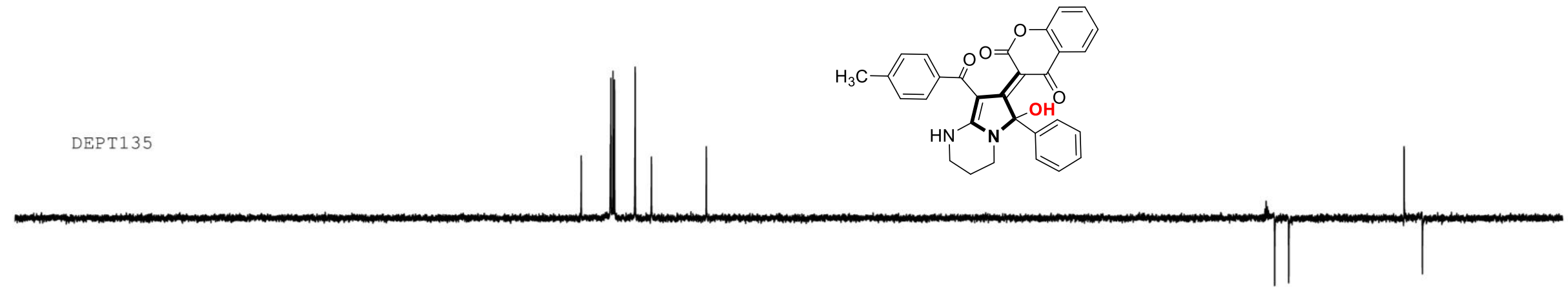

YUNNAN UNIVERSITY AVIIIHD500 ZQX-a-15

Jul27-2018-ziquanxing

C13CPD DMSO
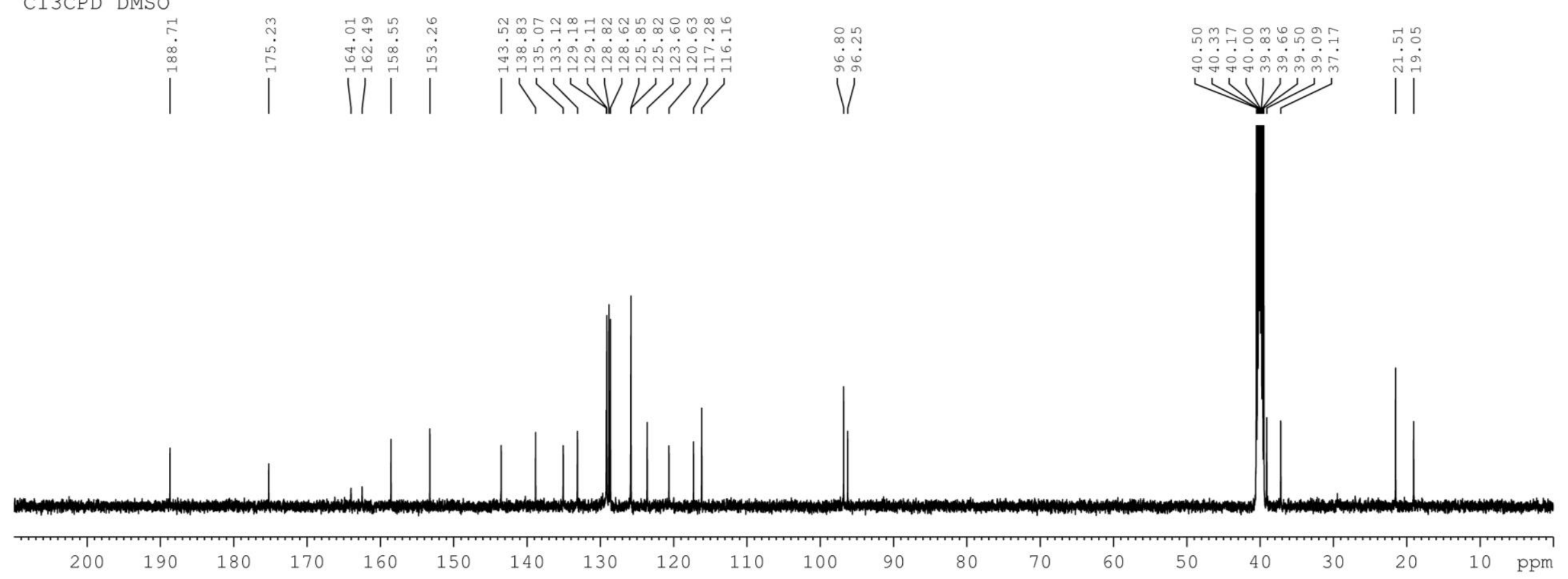

Figure S75. ${ }^{13} \mathrm{C}\left\{{ }^{1} \mathrm{H}\right\}$ NMR $\left(125 \mathrm{MHz}\right.$, DMSO- $\left.d_{6}\right)$ spectra of compound $\mathbf{6 n}$ 


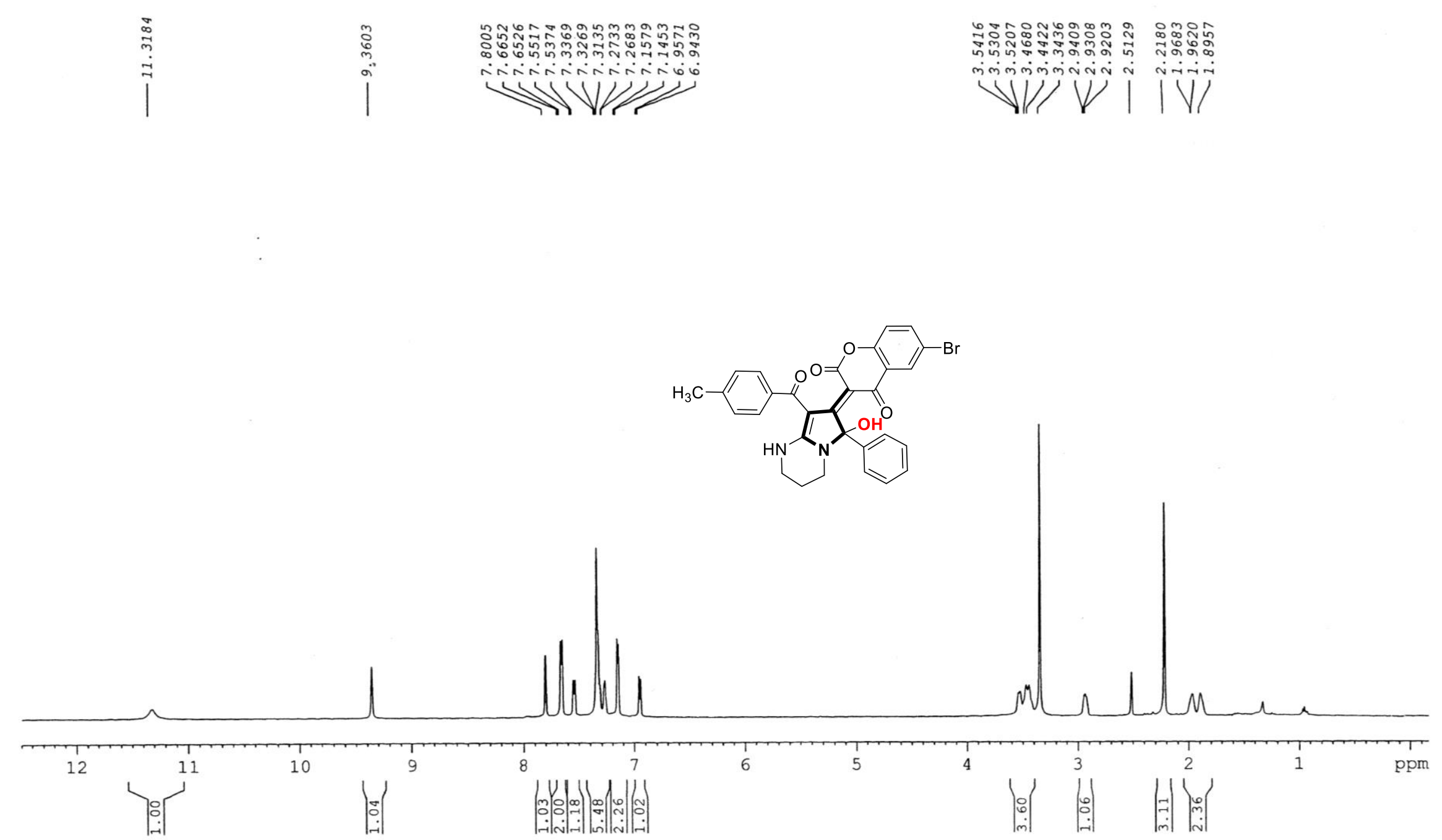

Figure S76. ${ }^{1} \mathrm{H}$ NMR $\left(600 \mathrm{MHz}, \mathrm{DMSO}-d_{6}\right)$ spectra of compound $\mathbf{6 o}$ 


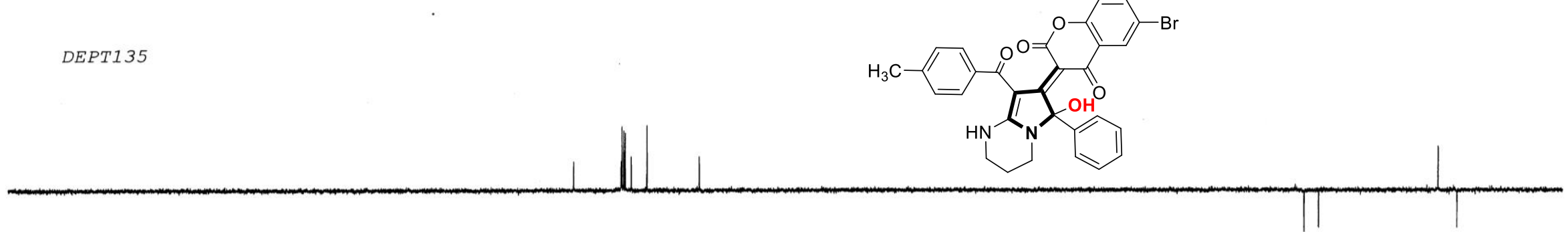

YUNNAN UNIVERSITY ASCEND AVIIIHD600 zQX-a-34 Aug10-2018-ziquanxing C13CPD DMS
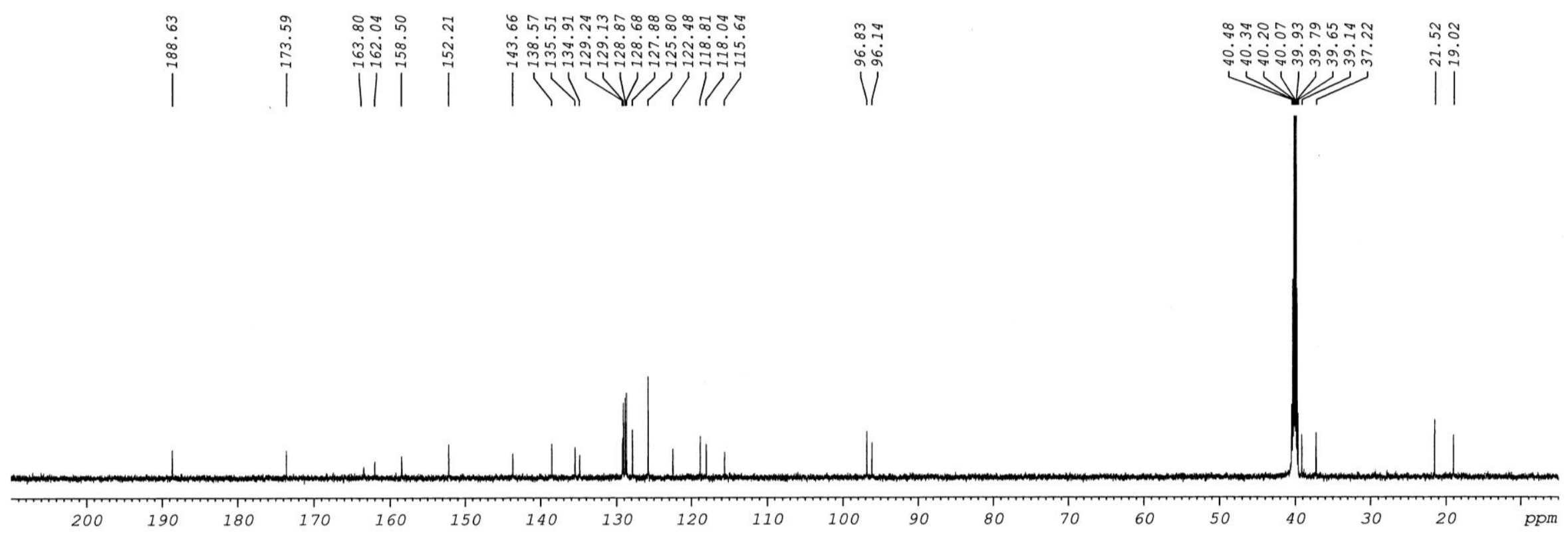

Figure S77. ${ }^{13} \mathrm{C}\left\{{ }^{1} \mathrm{H}\right\}$ NMR $\left(150 \mathrm{MHz}\right.$, DMSO- $\left.d_{6}\right)$ spectra of compound 60 


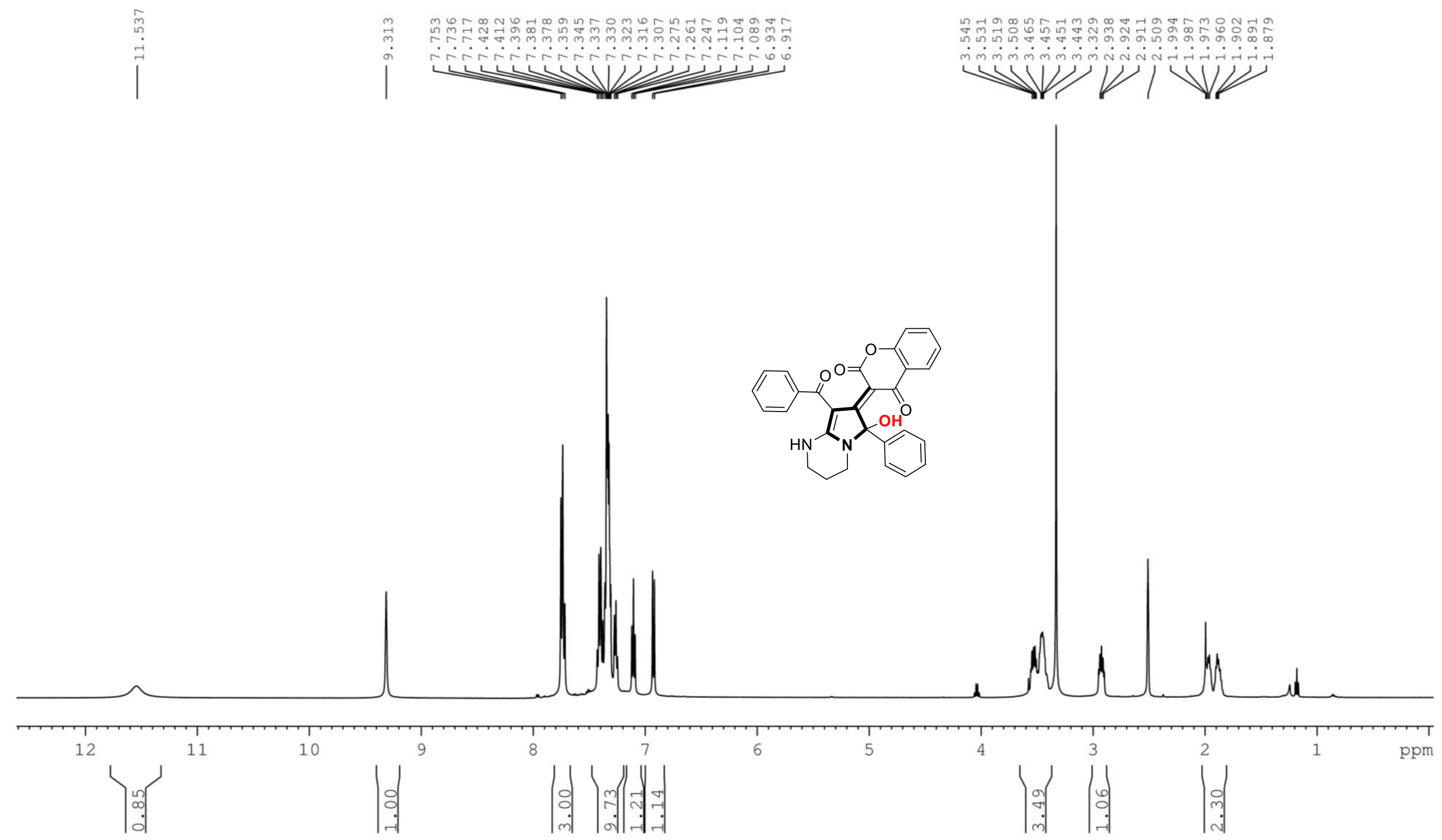

Figure S78. ${ }^{1} \mathrm{H}$ NMR $\left(500 \mathrm{MHz}, \mathrm{DMSO}-d_{6}\right)$ spectra of compound $\mathbf{6 p}$ 


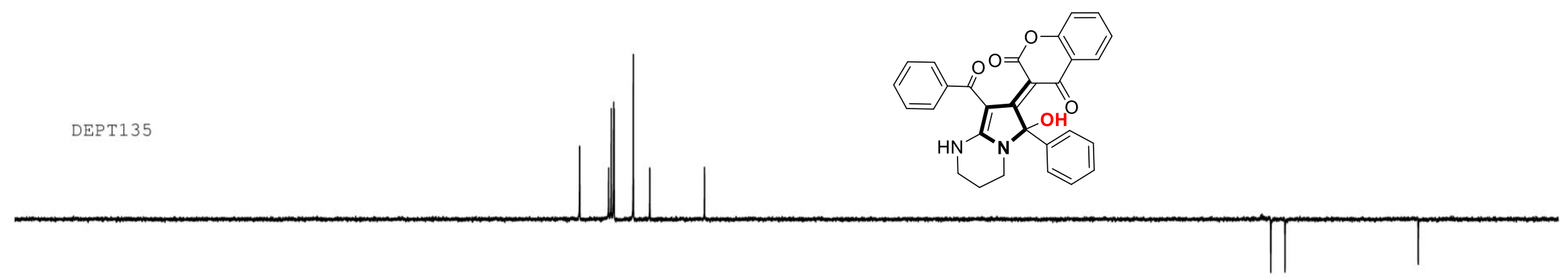

YUNNAN UNIVERSITY AVIIIHD500 ZQX-a-22-1 Aug01-2018-ziquanxing

C13CPD DMSO
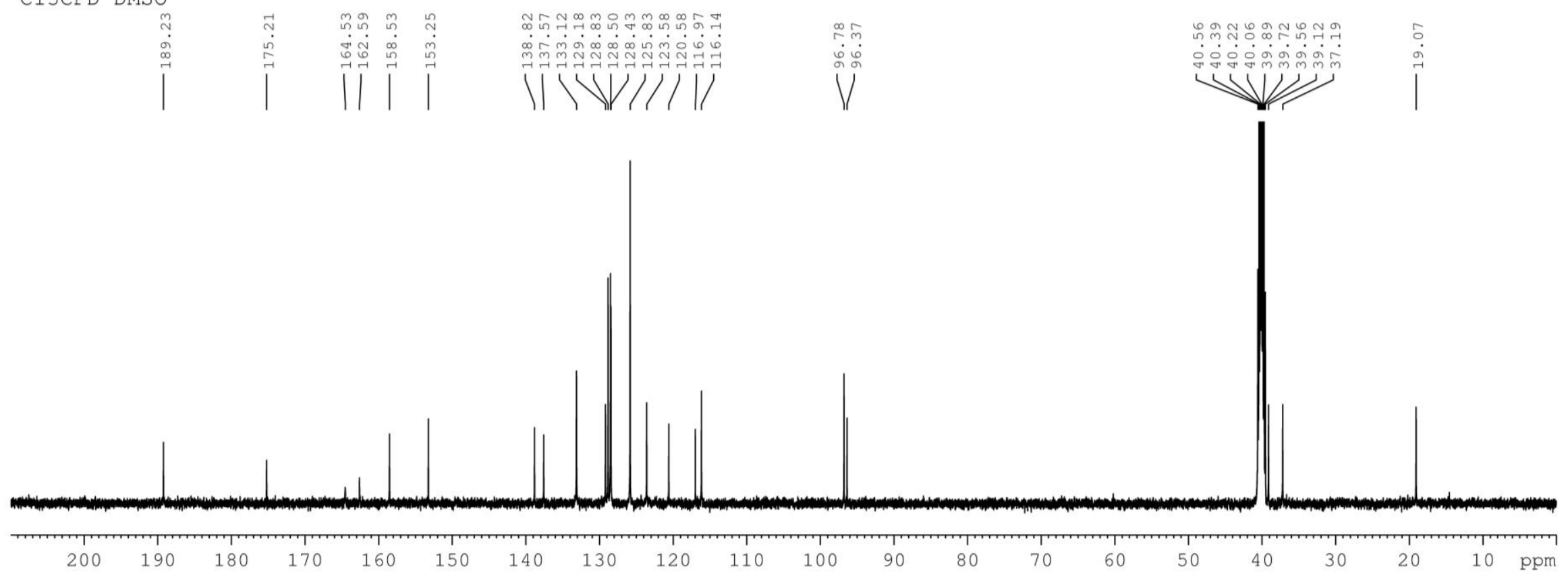

Figure S79. ${ }^{13} \mathrm{C}\left\{{ }^{1} \mathrm{H}\right\}$ NMR $\left(125 \mathrm{MHz}, \mathrm{DMSO}-d_{6}\right)$ spectra of compound $\mathbf{6 p}$ 
Vj
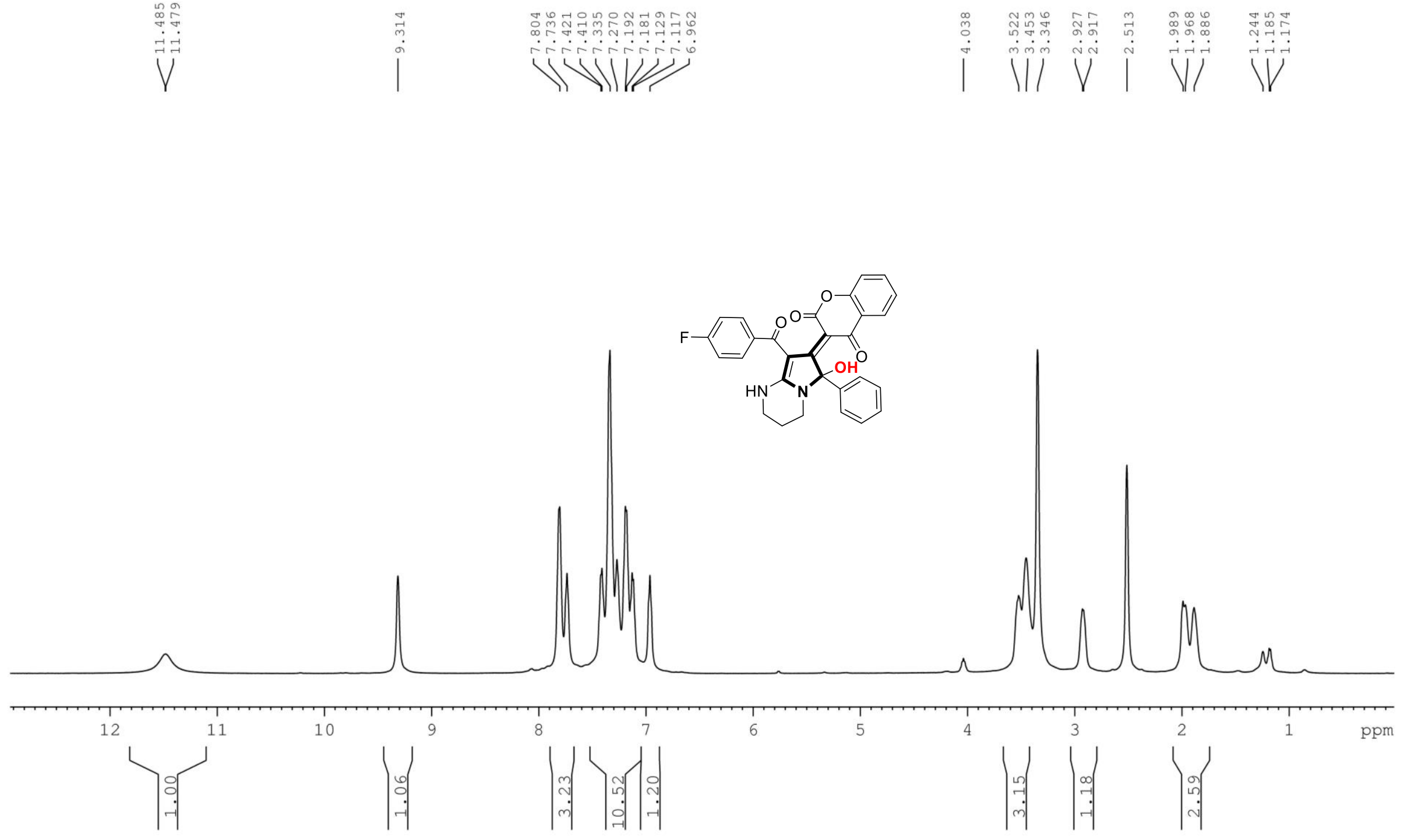

Figure S80. ${ }^{1} \mathrm{H}$ NMR $\left(500 \mathrm{MHz}, \mathrm{DMSO}-d_{6}\right)$ spectra of compound $\mathbf{6 q}$

S90 


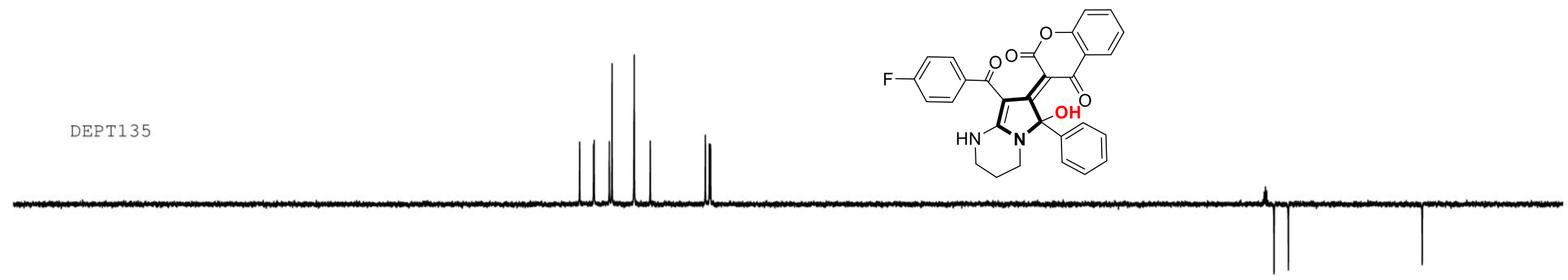

YUNNAN UNIVERSITY AVIIIHD500 ZOX-a-2 Jun27-2018-ziquanxing

C13CPD DMSO
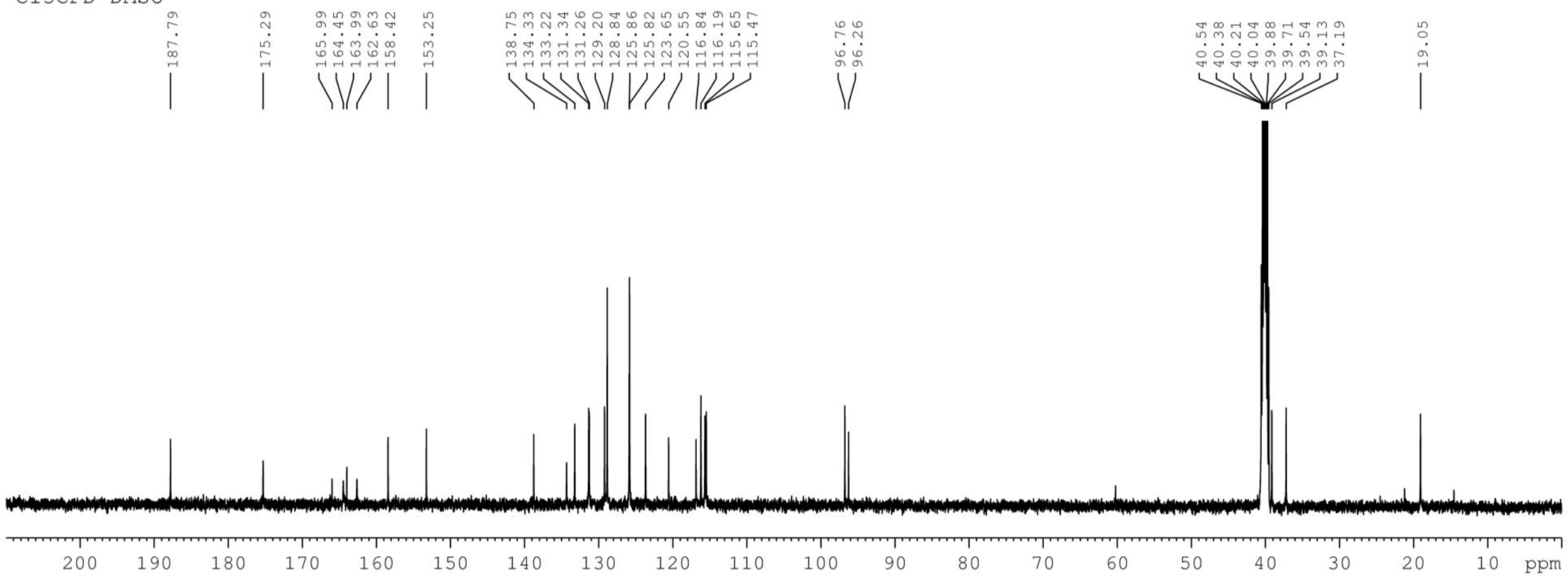

Figure S81. ${ }^{13} \mathrm{C}\left\{{ }^{1} \mathrm{H}\right\}$ NMR $\left(125 \mathrm{MHz}, \mathrm{DMSO}-d_{6}\right)$ spectra of compound $\mathbf{6 q}$ 


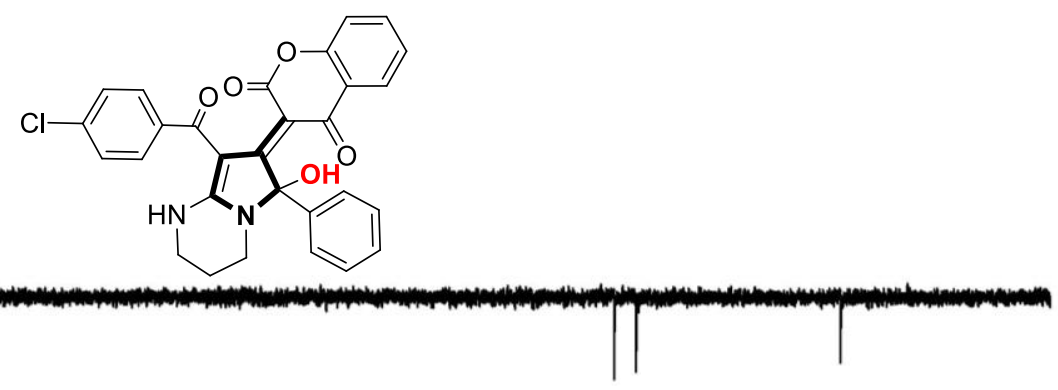

YUNNAN UNIVERSITY ASCEND AVIIIHD600 ZQX-A-1 Mar23-2019-ziquanxing + C13CPD DMSO
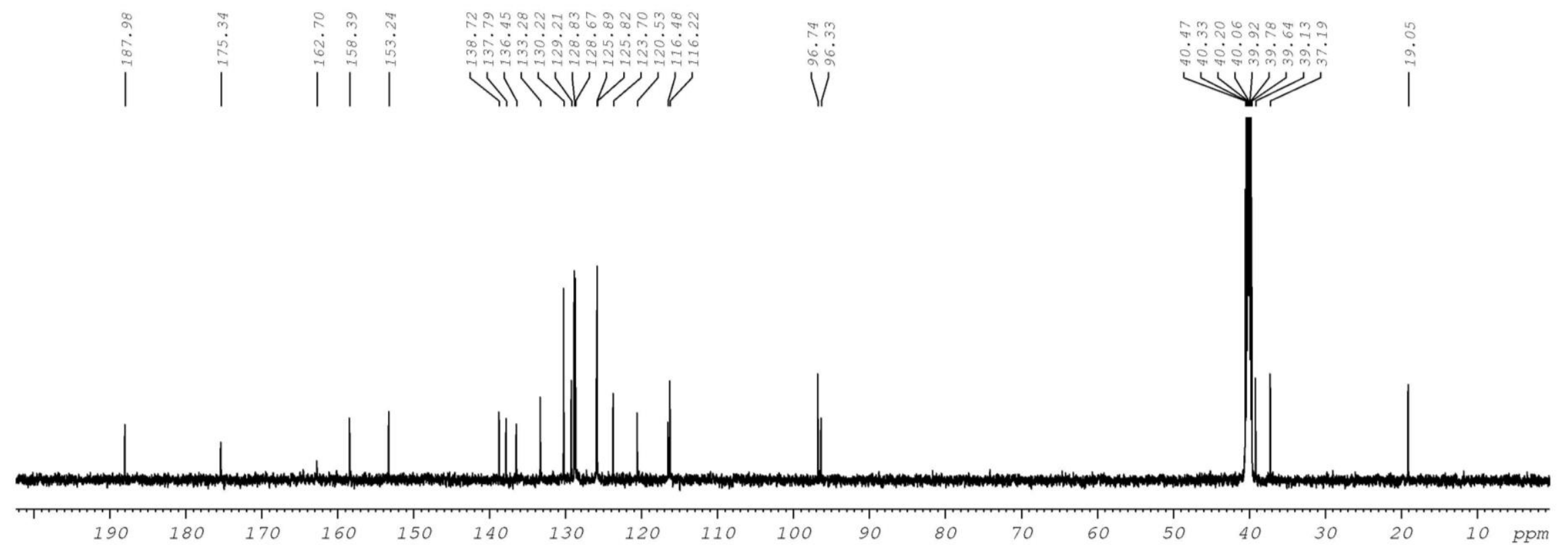

Figure S83. ${ }^{13} \mathrm{C}\left\{{ }^{1} \mathrm{H}\right\}$ NMR (150 MHz, DMSO- $\left.d_{6}\right)$ spectra of compound $6 \mathbf{r}$ 


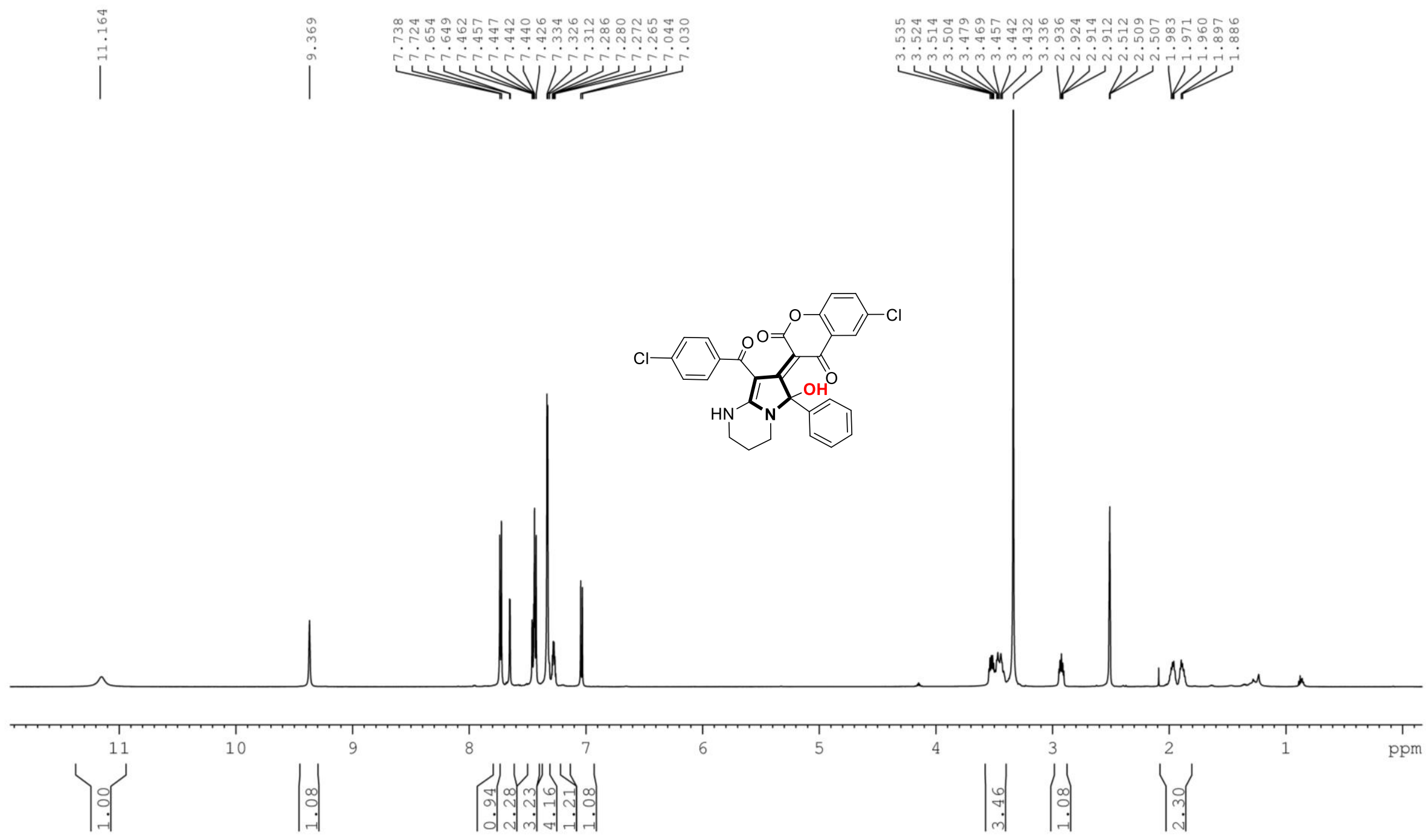

Figure S84. ${ }^{1} \mathrm{H}$ NMR $\left(600 \mathrm{MHz}, \mathrm{DMSO}-d_{6}\right)$ spectra of compound $\mathbf{6 s}$ 


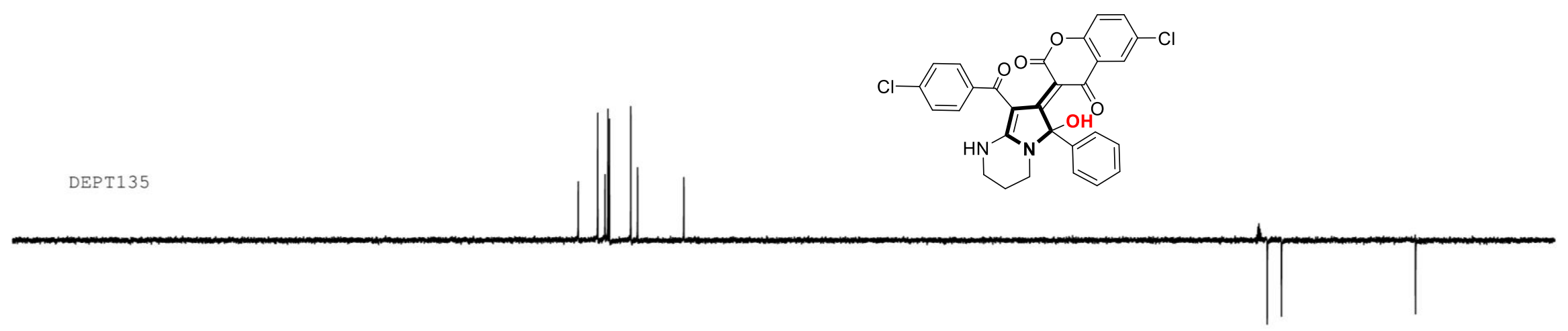

YUNNAN UNIVERSITY ASCEND AVIIIHD600 ZOX-a-25 Aug28-2018-ziquanxing C13CPD DMSO
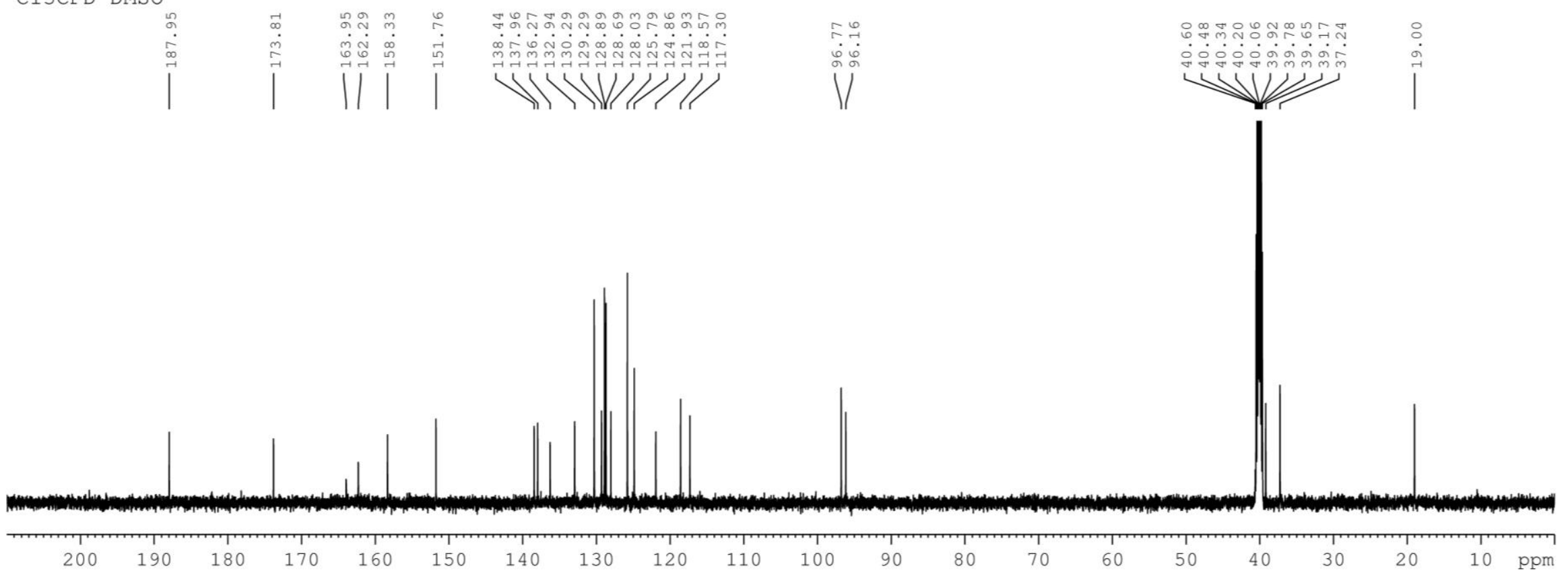

Figure S85. ${ }^{13} \mathrm{C}\left\{{ }^{1} \mathrm{H}\right\}$ NMR $\left(150 \mathrm{MHz}\right.$, DMSO- $\left.d_{6}\right)$ spectra of compound $\mathbf{6 s}$ 


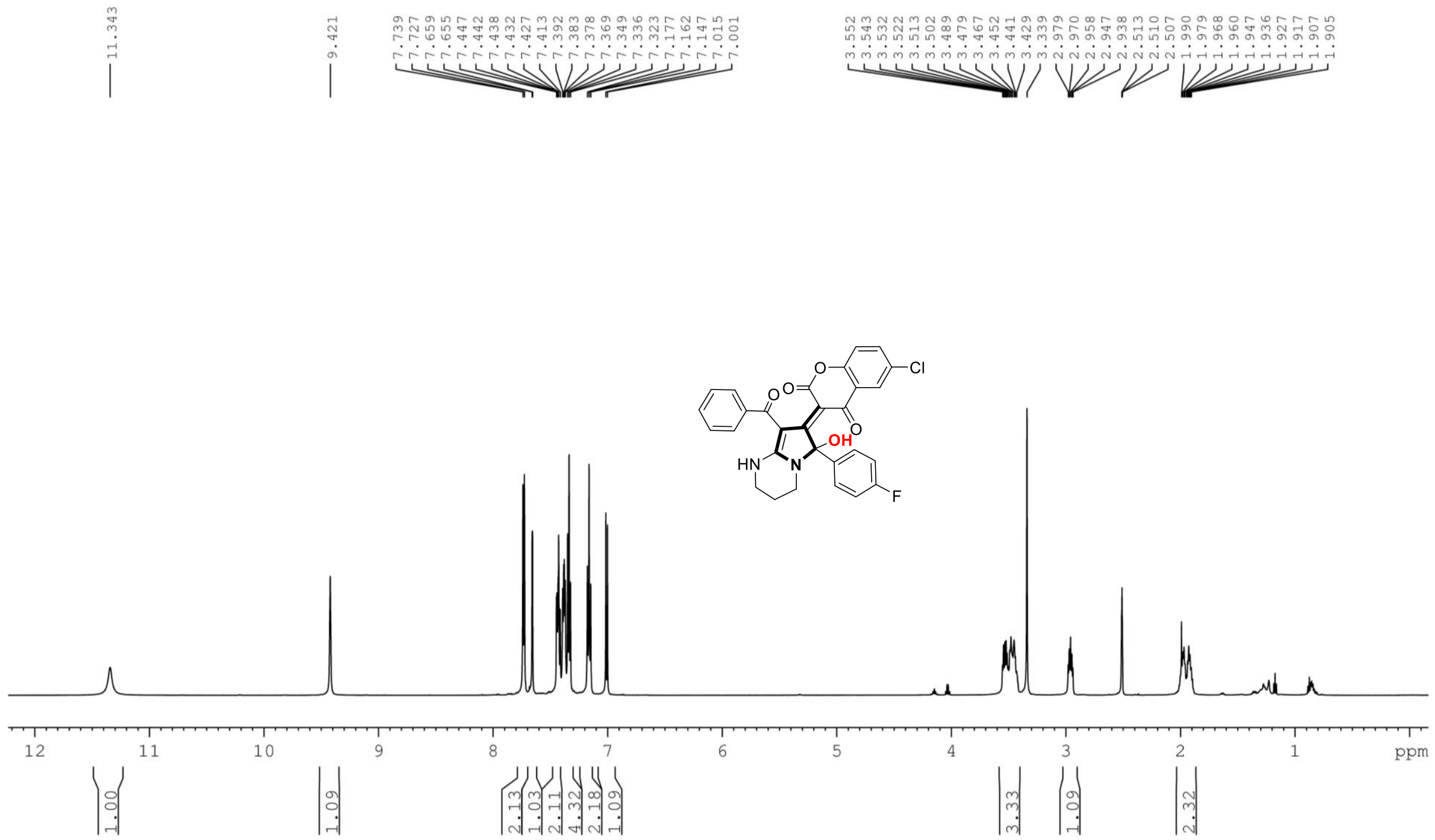

Figure S86. ${ }^{1} \mathrm{H}$ NMR $\left(600 \mathrm{MHz}, \mathrm{DMSO}-d_{6}\right)$ spectra of compound $6 \mathbf{t}$ 


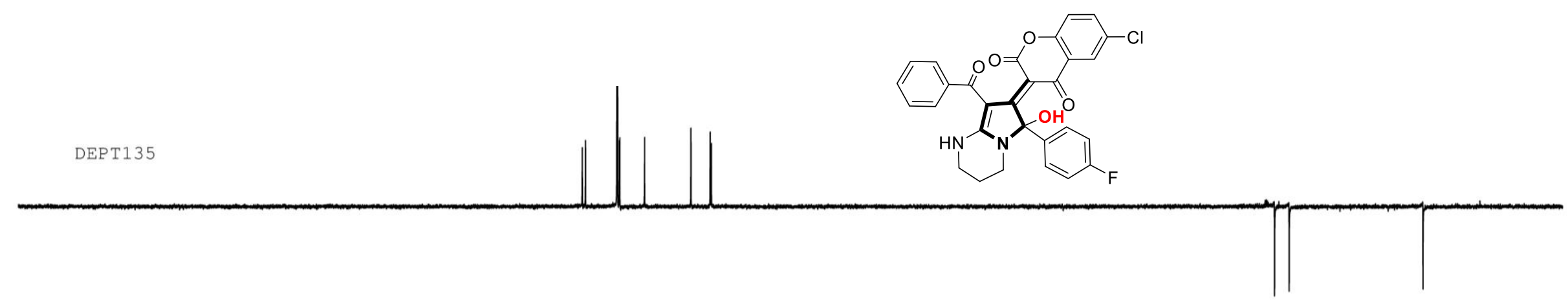

YUNNAN UNIVERSITY ASCEND AVIIIHD600 ZQX-a-30

Aug23-2018-ziquanxing

C13CPD DMSO
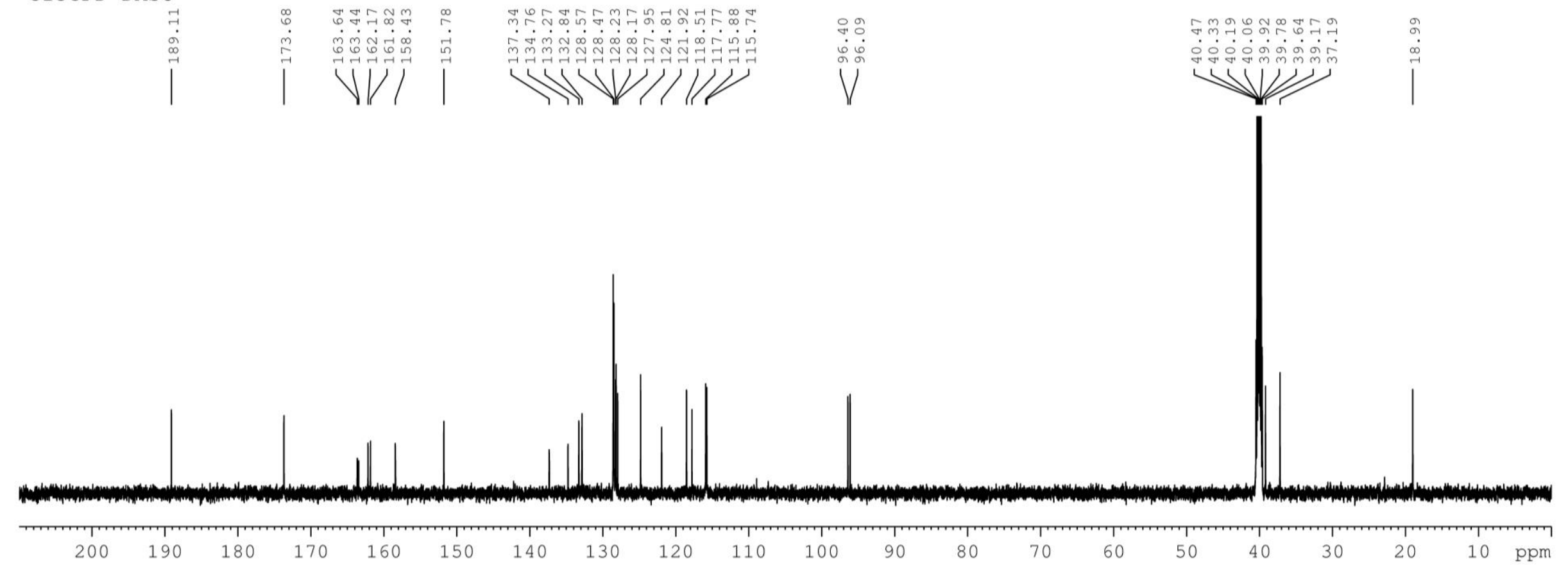

Figure S87. ${ }^{13} \mathrm{C}\left\{{ }^{1} \mathrm{H}\right\}$ NMR $\left(150 \mathrm{MHz}, \mathrm{DMSO}-d_{6}\right)$ spectra of compound $6 \mathbf{t}$ 


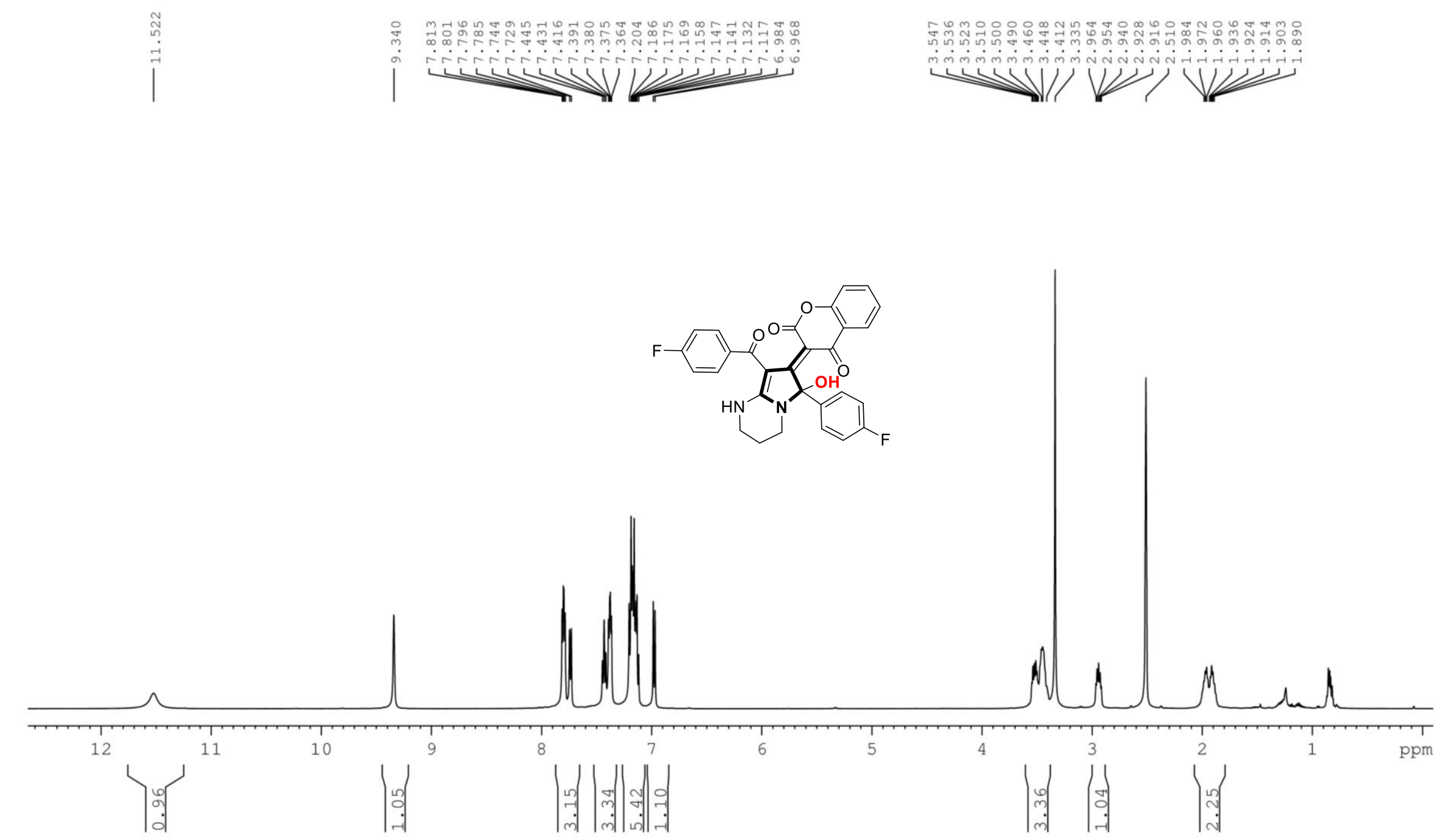

Figure S88. ${ }^{1} \mathrm{H}$ NMR $\left(500 \mathrm{MHz}, \mathrm{DMSO}-d_{6}\right)$ spectra of compound $\mathbf{6 u}$ 


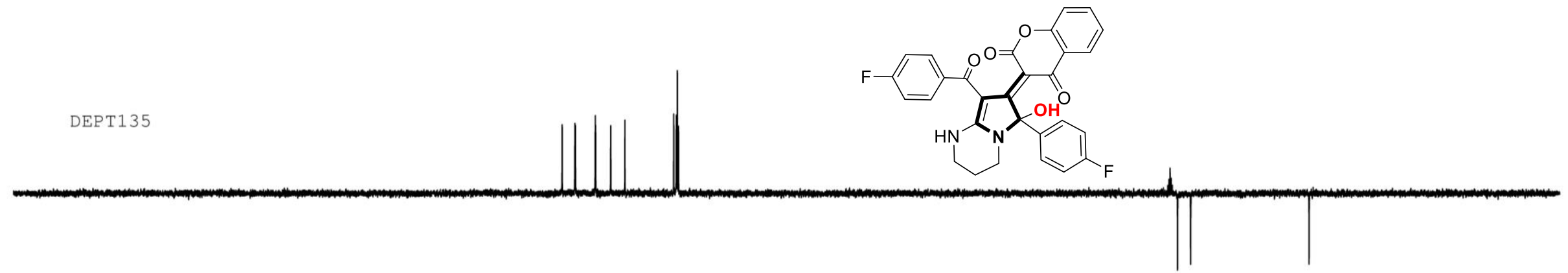

YUNNAN UNIVERSITY AVIIIHD500 ZQX-a-4 Jun05-2018-ziquanxing

C13CPD Dioxane
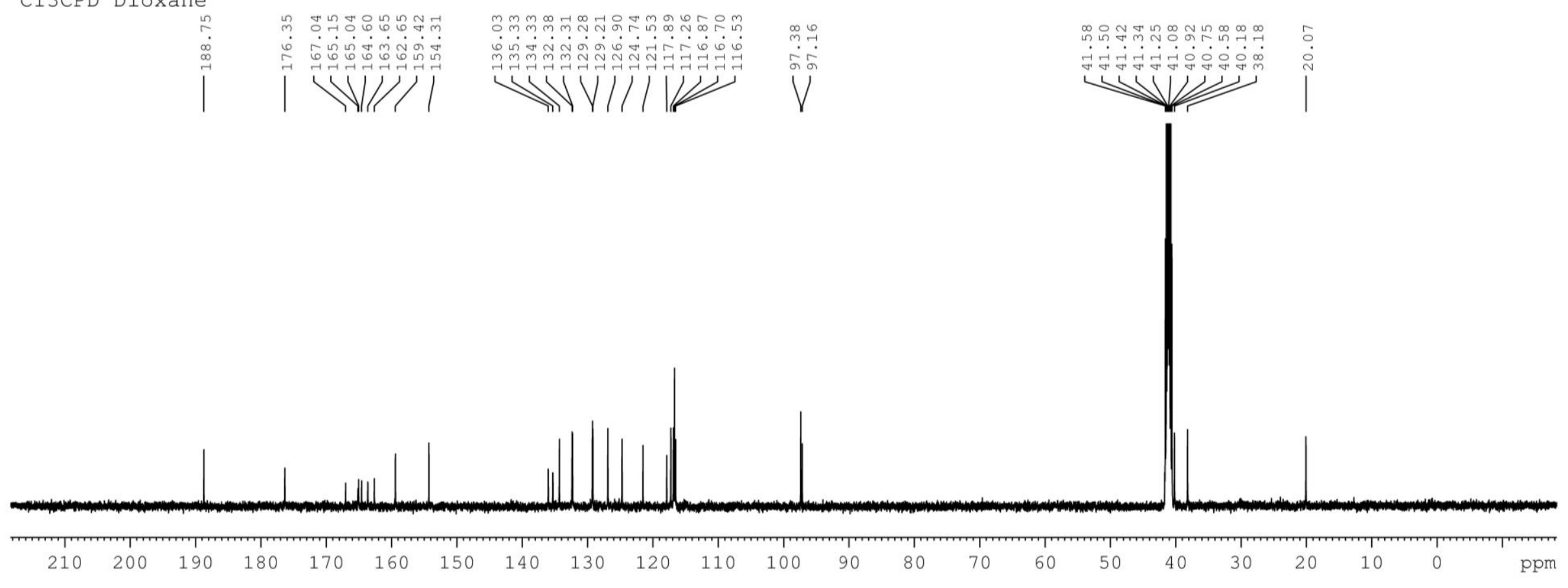

Figure S89. ${ }^{13} \mathrm{C}\left\{{ }^{1} \mathrm{H}\right\}$ NMR $\left(125 \mathrm{MHz}, \mathrm{DMSO}-d_{6}\right)$ spectra of compound $6 \mathbf{u}$ 


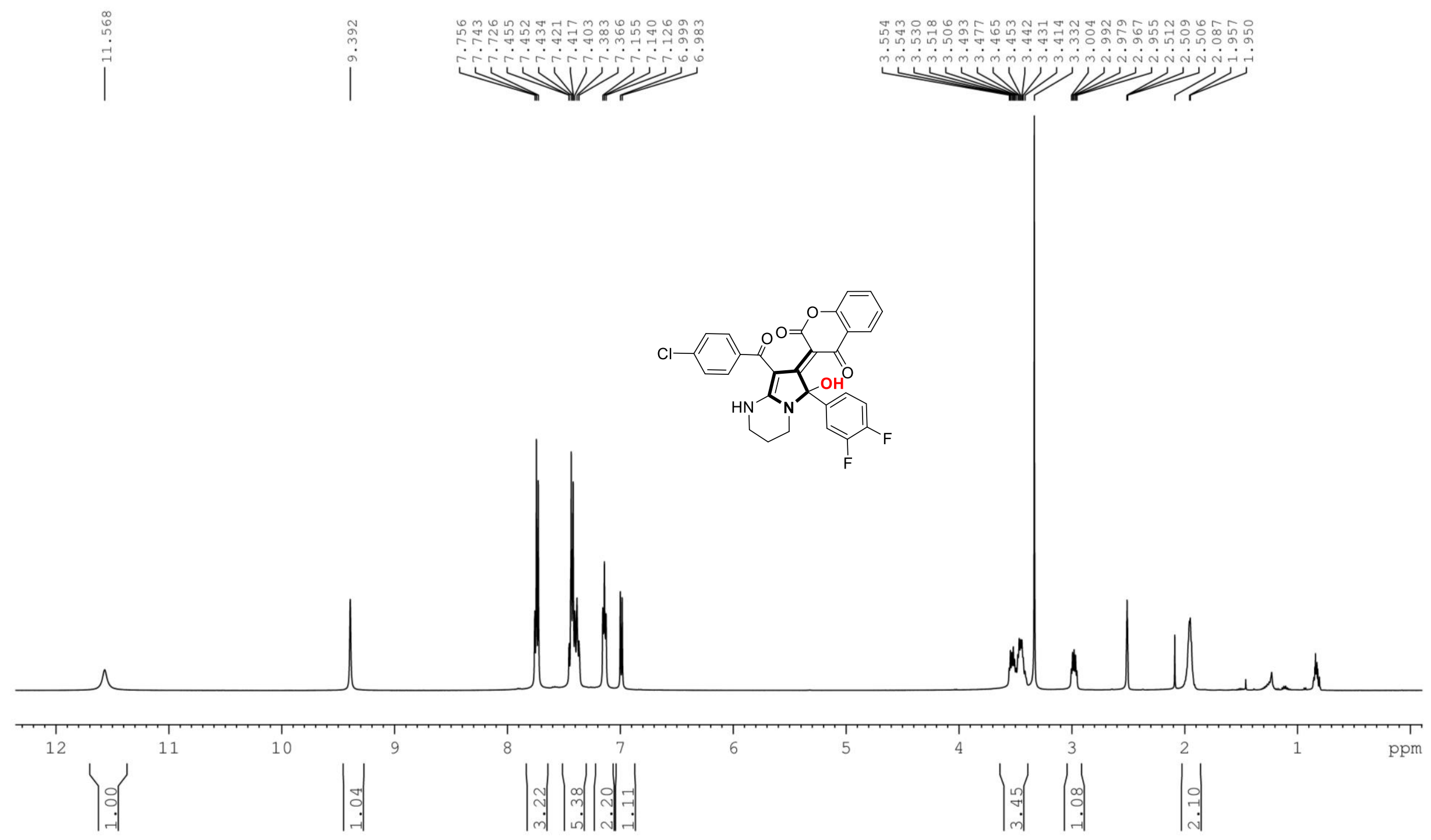

Figure S90. ${ }^{1} \mathrm{H}$ NMR $\left(500 \mathrm{MHz}\right.$, DMSO- $\left.d_{6}\right)$ spectra of compound $\mathbf{6 v}$ 


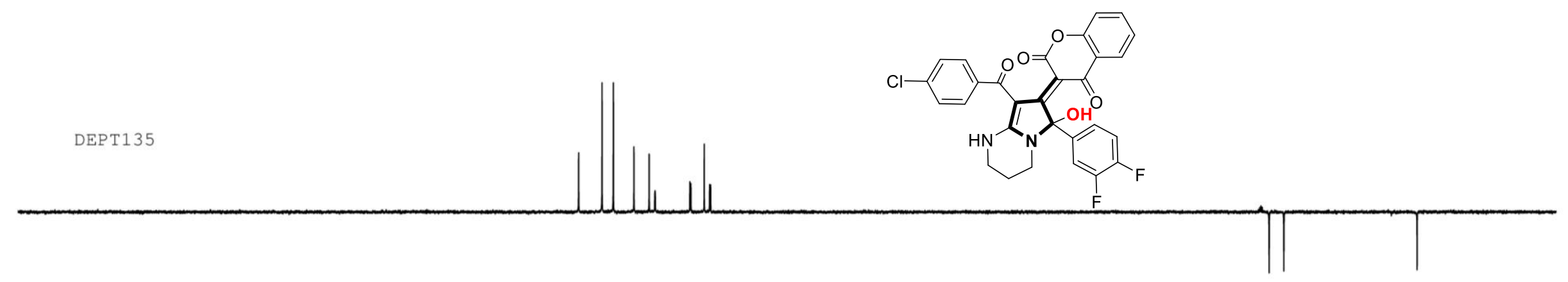

YUNNAN UNIVERSITY AVIIIHD500 ZQX-a-18-1

Aug01-2018-ziquanxing

C13CPD DMSO
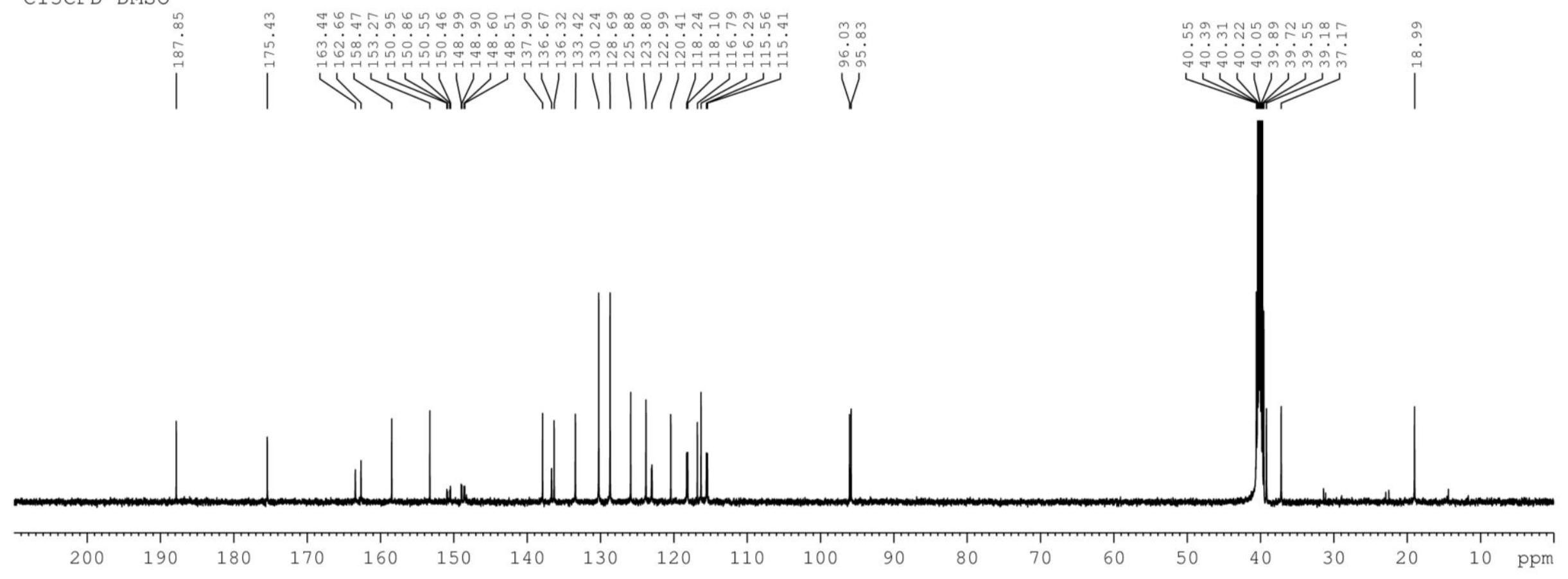

Figure S91. ${ }^{13} \mathrm{C}\left\{{ }^{1} \mathrm{H}\right\}$ NMR $\left(125 \mathrm{MHz}, \mathrm{DMSO}-d_{6}\right)$ spectra of compound $\mathbf{6 v}$ 


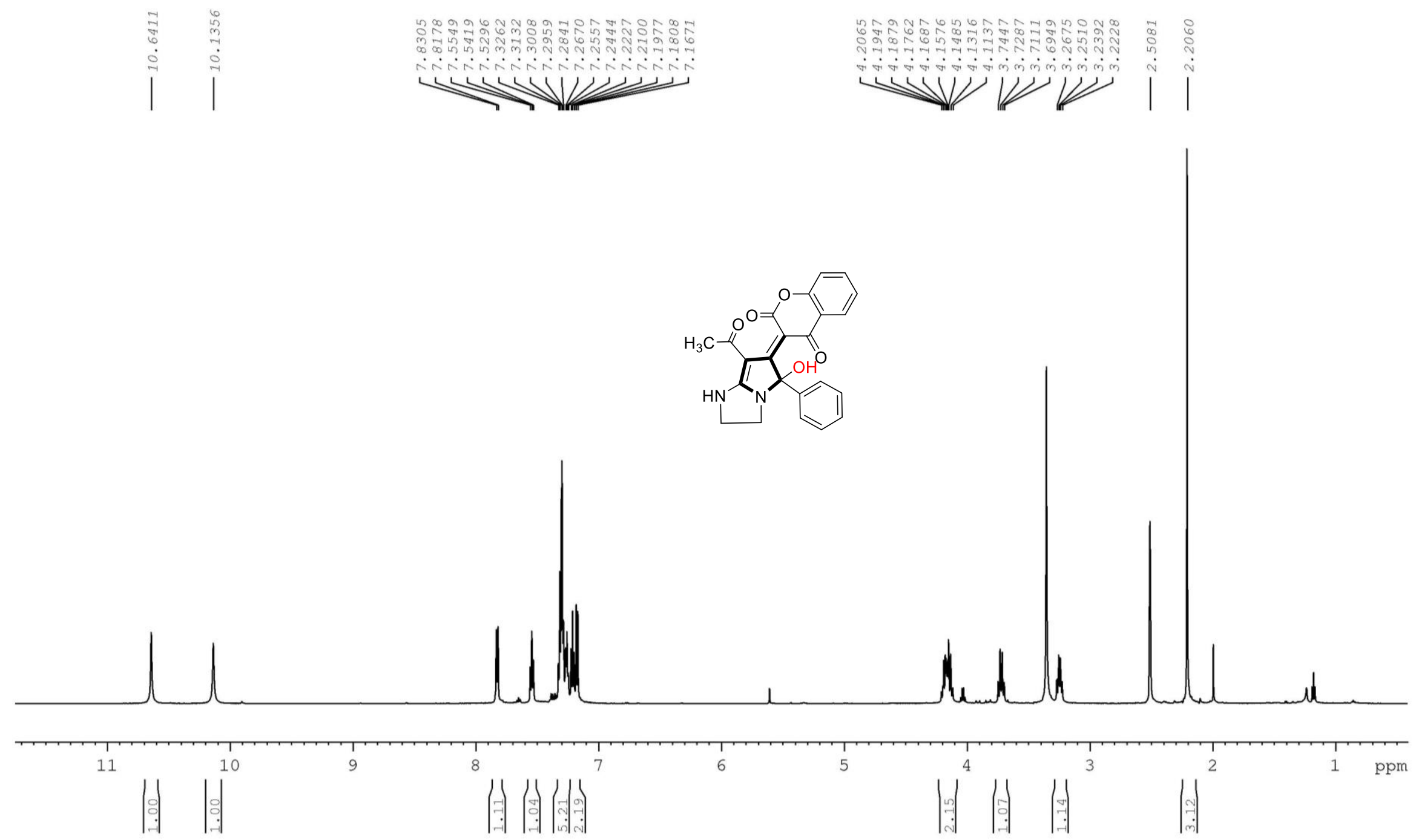

Figure S92. ${ }^{1} \mathrm{H}$ NMR $\left(500 \mathrm{MHz}, \mathrm{DMSO}-d_{6}\right)$ spectra of compound $\mathbf{6} \mathbf{w}$ 


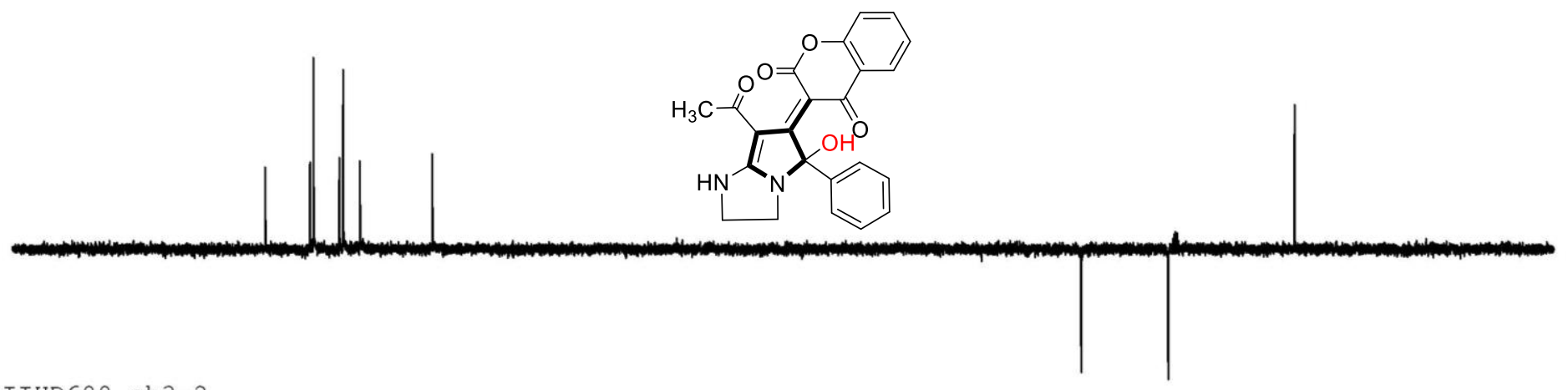

YUNNAN UNIVERSITY ASCEND AVIIIHD600 ch3-2
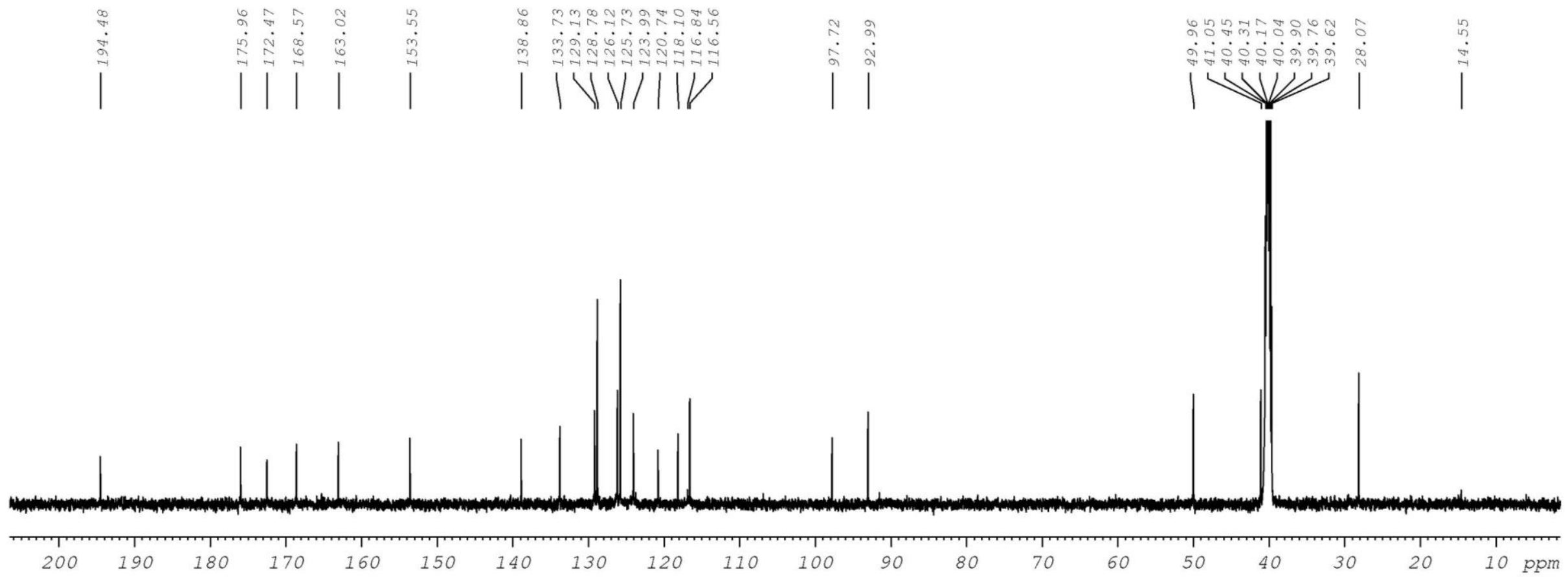

Figure S93. ${ }^{13} \mathrm{C}\left\{{ }^{1} \mathrm{H}\right\}$ NMR $(125 \mathrm{MHz}$, DMSO-d 6 ) spectra of compound $6 \mathbf{w}$ 


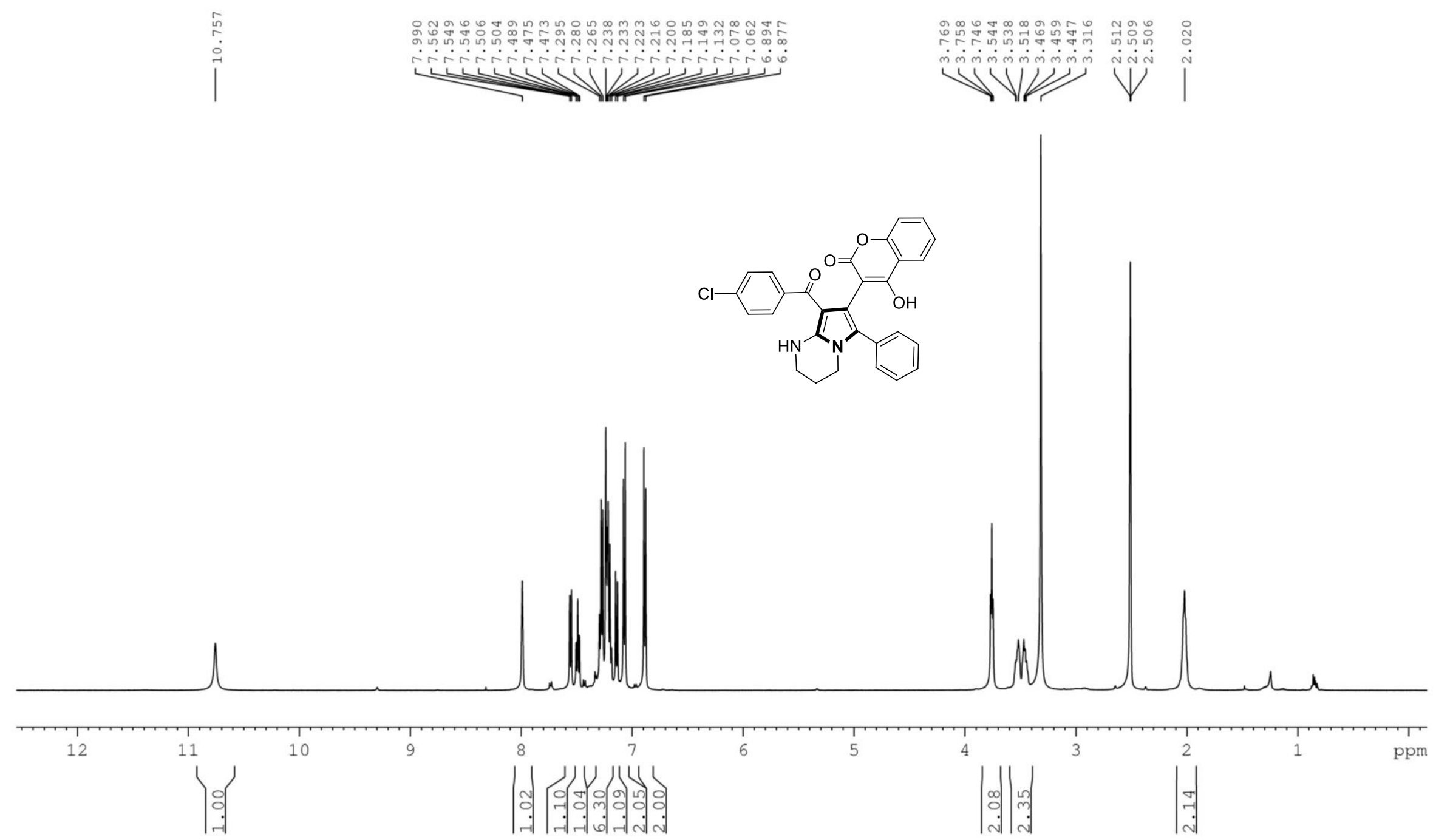

Figure S94. ${ }^{1} \mathrm{H}$ NMR $\left(500 \mathrm{MHz}, \mathrm{DMSO}-d_{6}\right)$ spectra of compound $7 \mathbf{r}$ 


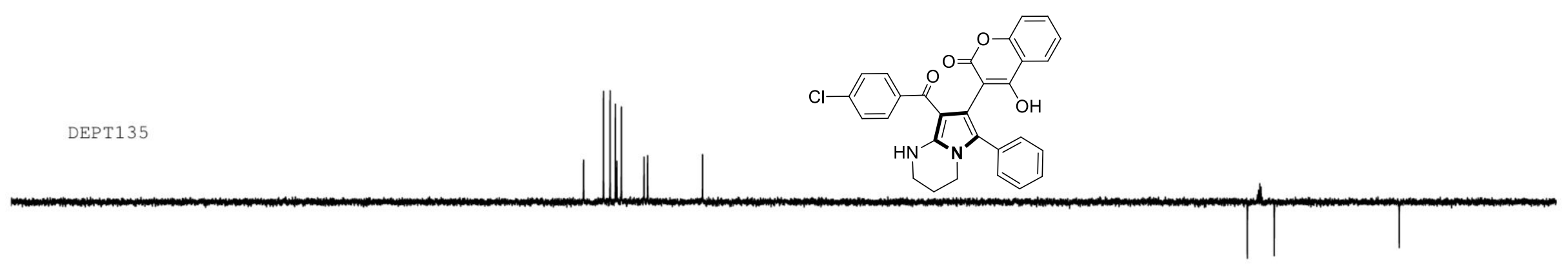

YUNNAN UNIVERSITY AVIIIHD500 ZOX-a-1 May30-2018-ziquanxing

C13CPD DMSO
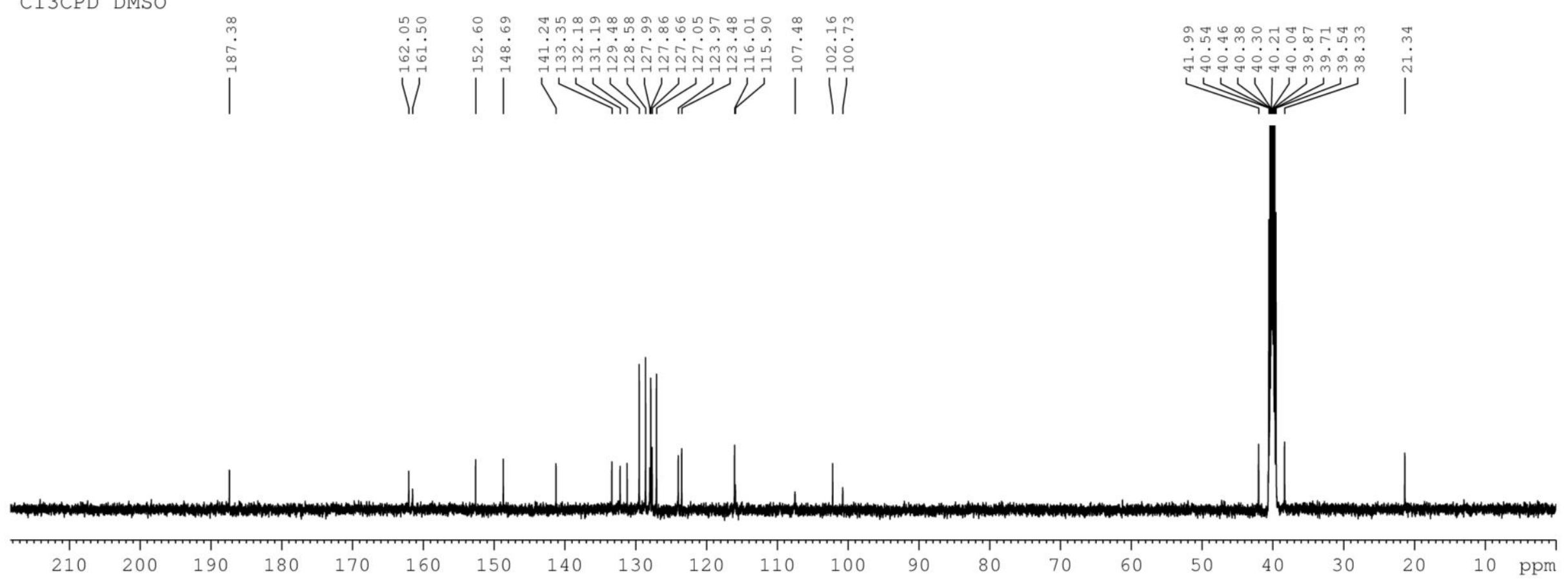

Figure S95. ${ }^{13} \mathrm{C}\left\{{ }^{1} \mathrm{H}\right\}$ NMR (125 MHz, DMSO- $\left.d_{6}\right)$ spectra of compound $7 \mathbf{r}$ 
$75 \mathrm{\exists}$

70

65

60

55

50

$45-$

40 牲

35

30 手

25

20

15

$$
\text { } 0 .
$$
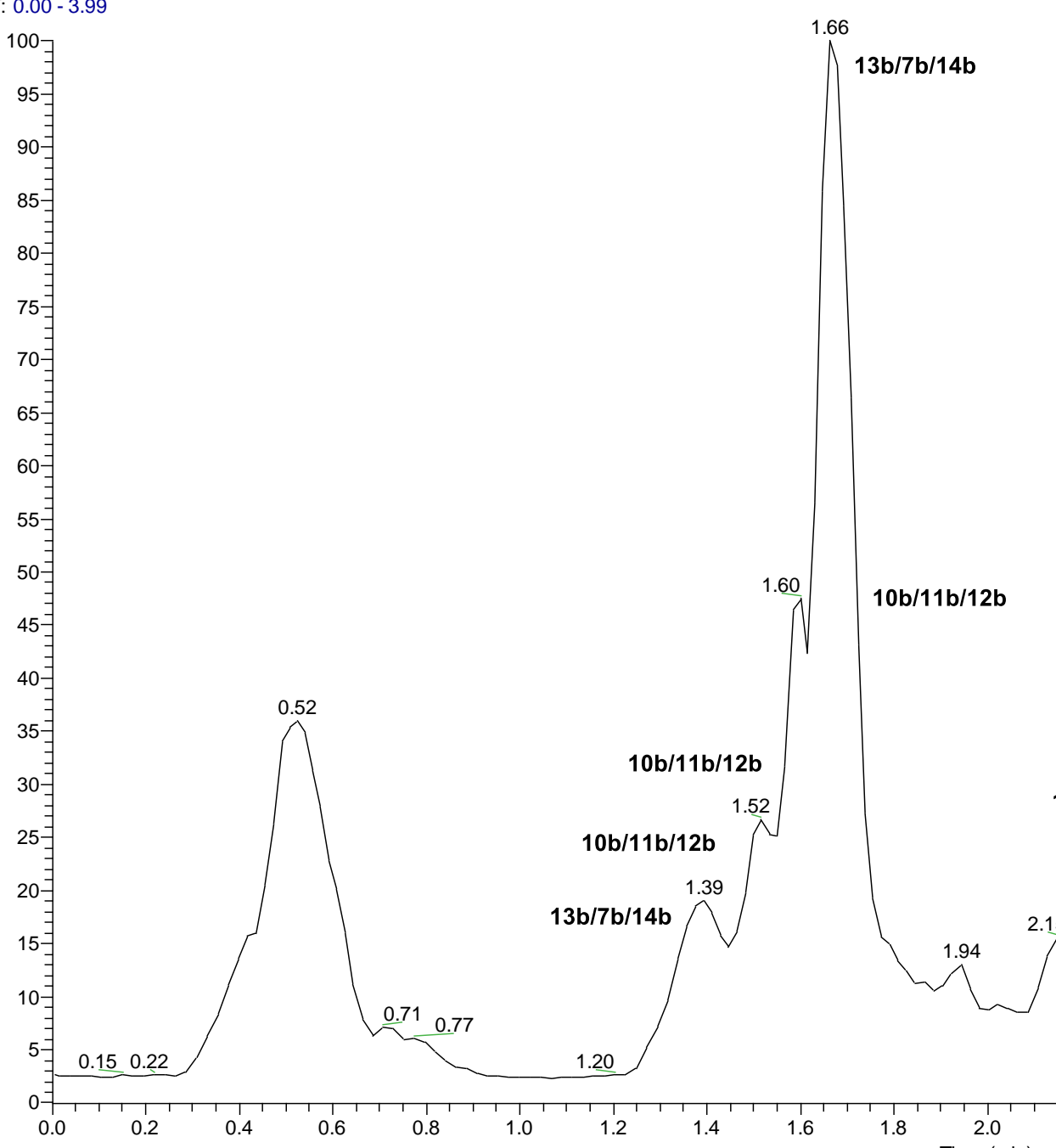

$13 b / 7 b / 14 b$

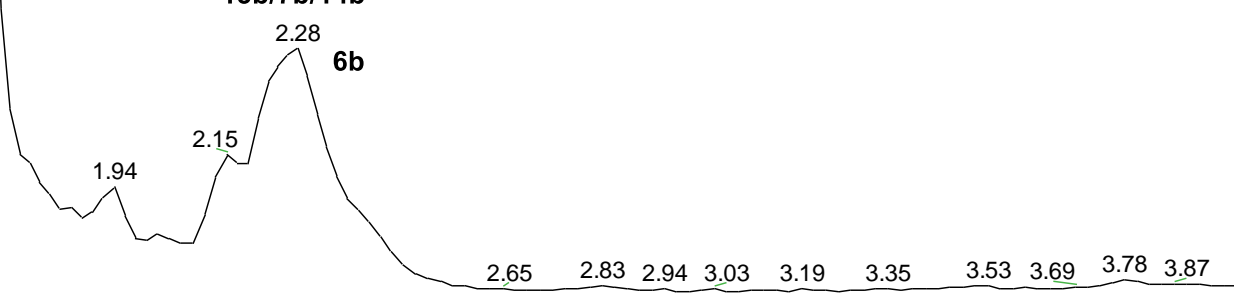

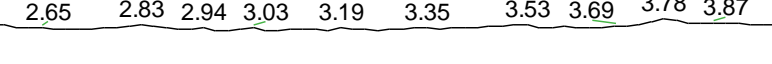

Figure S96. HPLC of the reaction mixture 
08-08 \#75 RT: 1.53 AV: $1 \quad$ NL: $1.03 E 5$
T: FTMS + c ESI Full ms [200.00-1100.00]

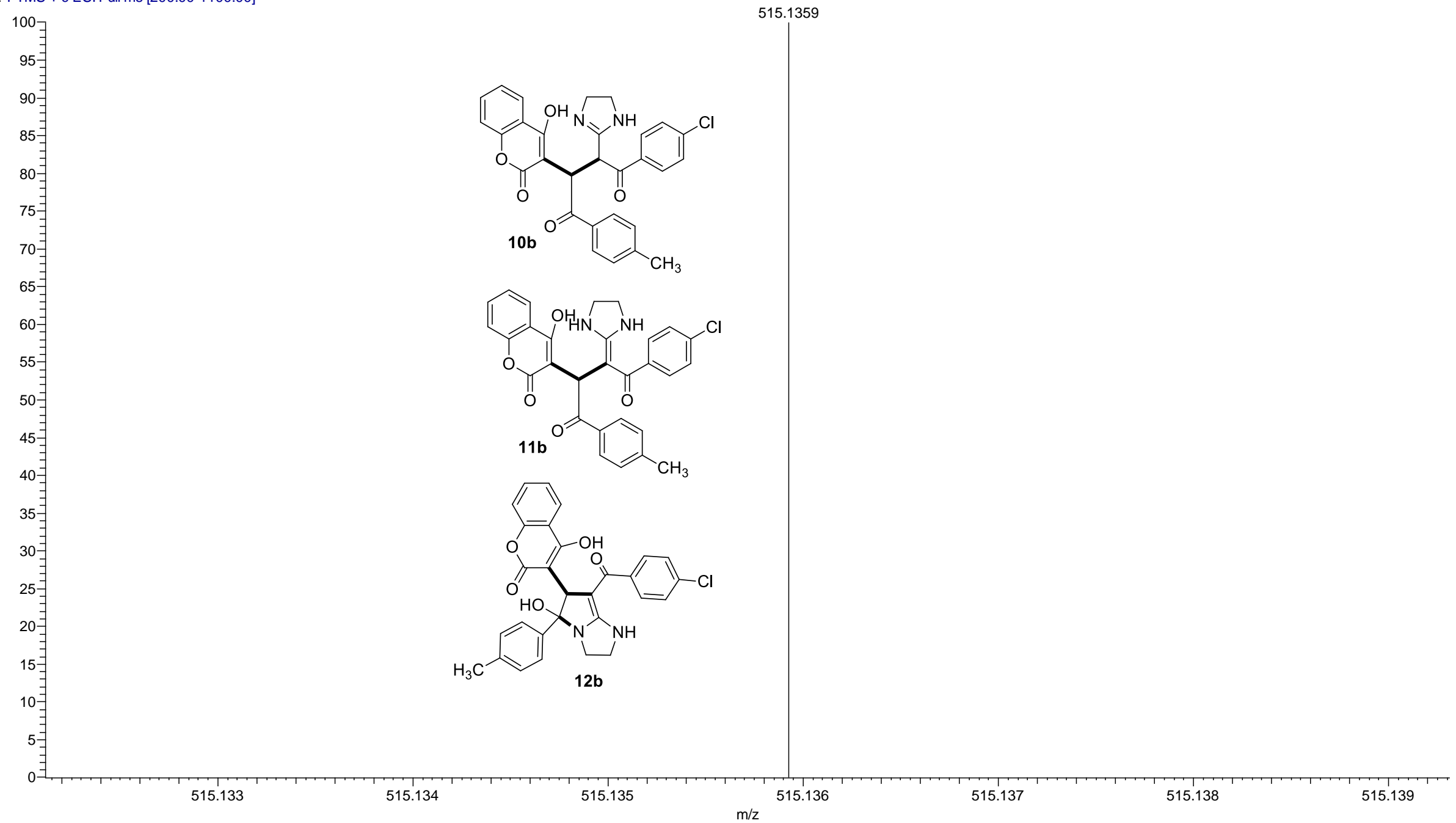

Figure S97. HRMS of intermediate 10b/11b/12b 


$$
\begin{gathered}
{ }^{100} \\
95 \\
90 \\
90 \\
85 \\
80 \\
75 \\
70 \\
70 \\
65 \\
60 \\
65 \\
55 \\
50= \\
45 \\
40= \\
35 \\
30 \\
25 \\
20 \\
20= \\
15 \\
10= \\
5 \\
5 \\
0
\end{gathered}
$$

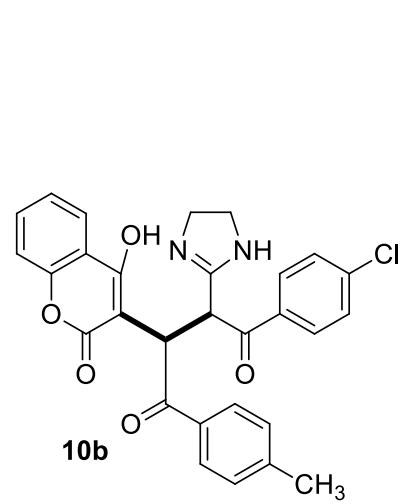

515.1356
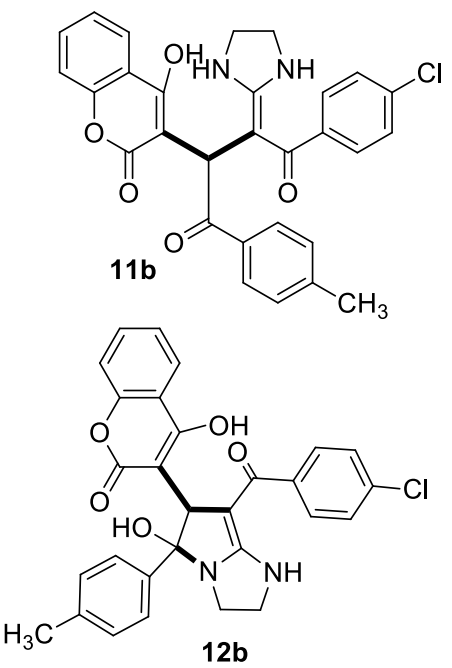

515.133

515.134

515.135

515.137

515.138

515.139

Figure S98. HRMS of intermediate 10b/11b/12b 


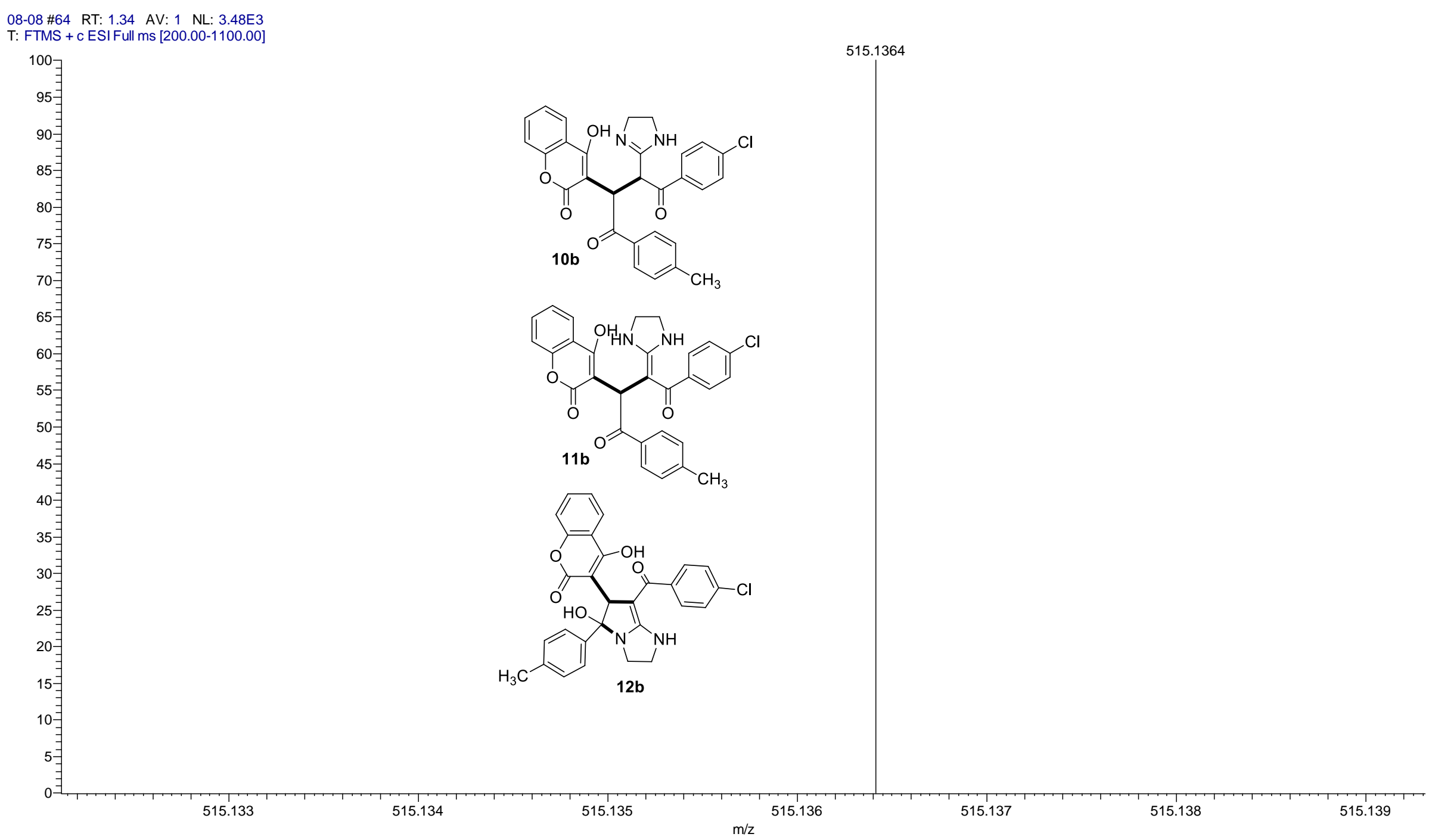

Figure S99. HRMS of intermediate $\mathbf{1 0 b} / \mathbf{1 1 b} / \mathbf{1 2 b}$ 


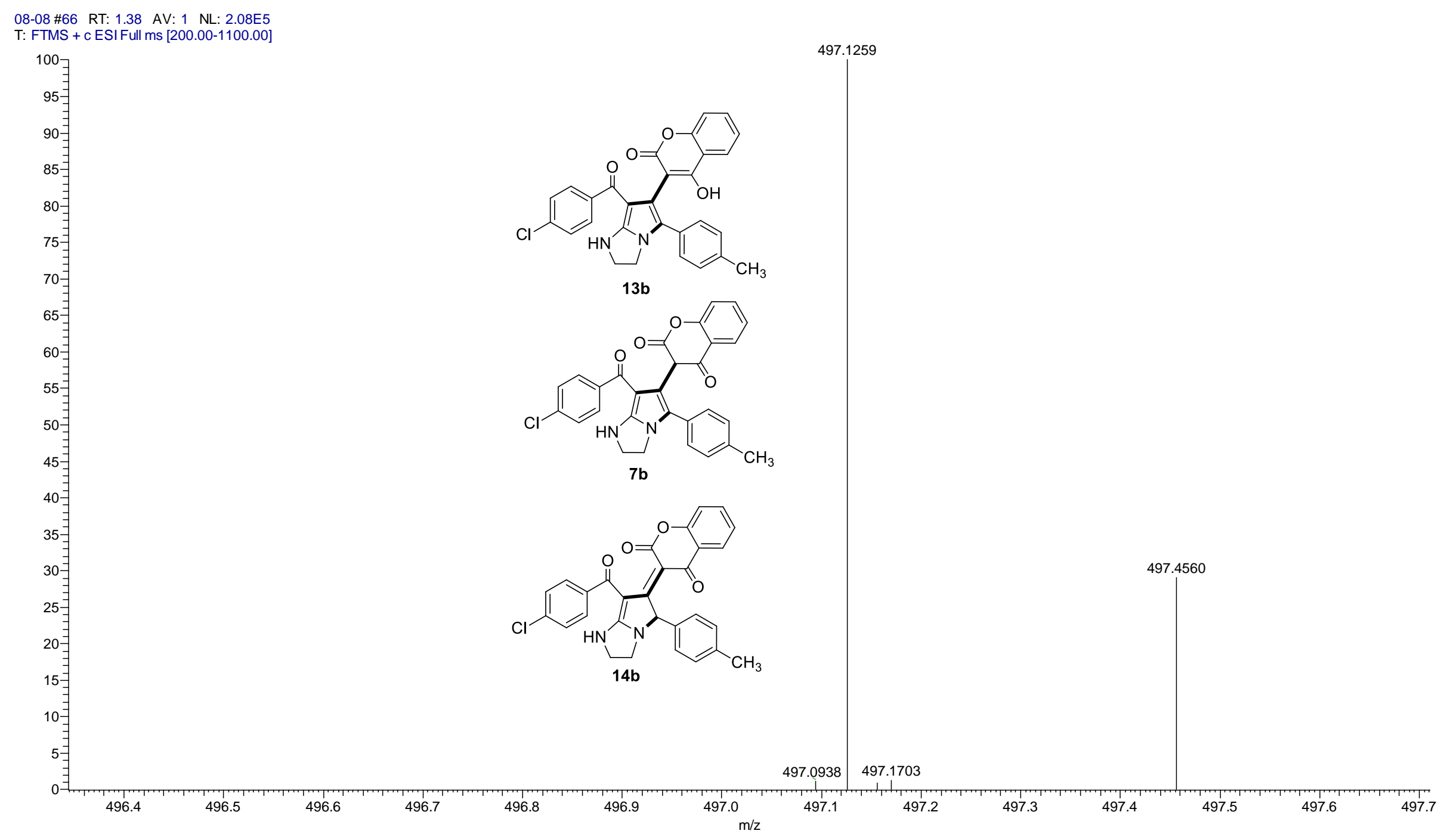

Figure S100. HRMS of intermediate $13 b / 7 b / 14 b$ 
08-08 \#83 RT: 1.66 AV: $1 \quad$ NL: $2.60 E 7$

T: FTMS + c ESI Full ms [200.00-1100.00]

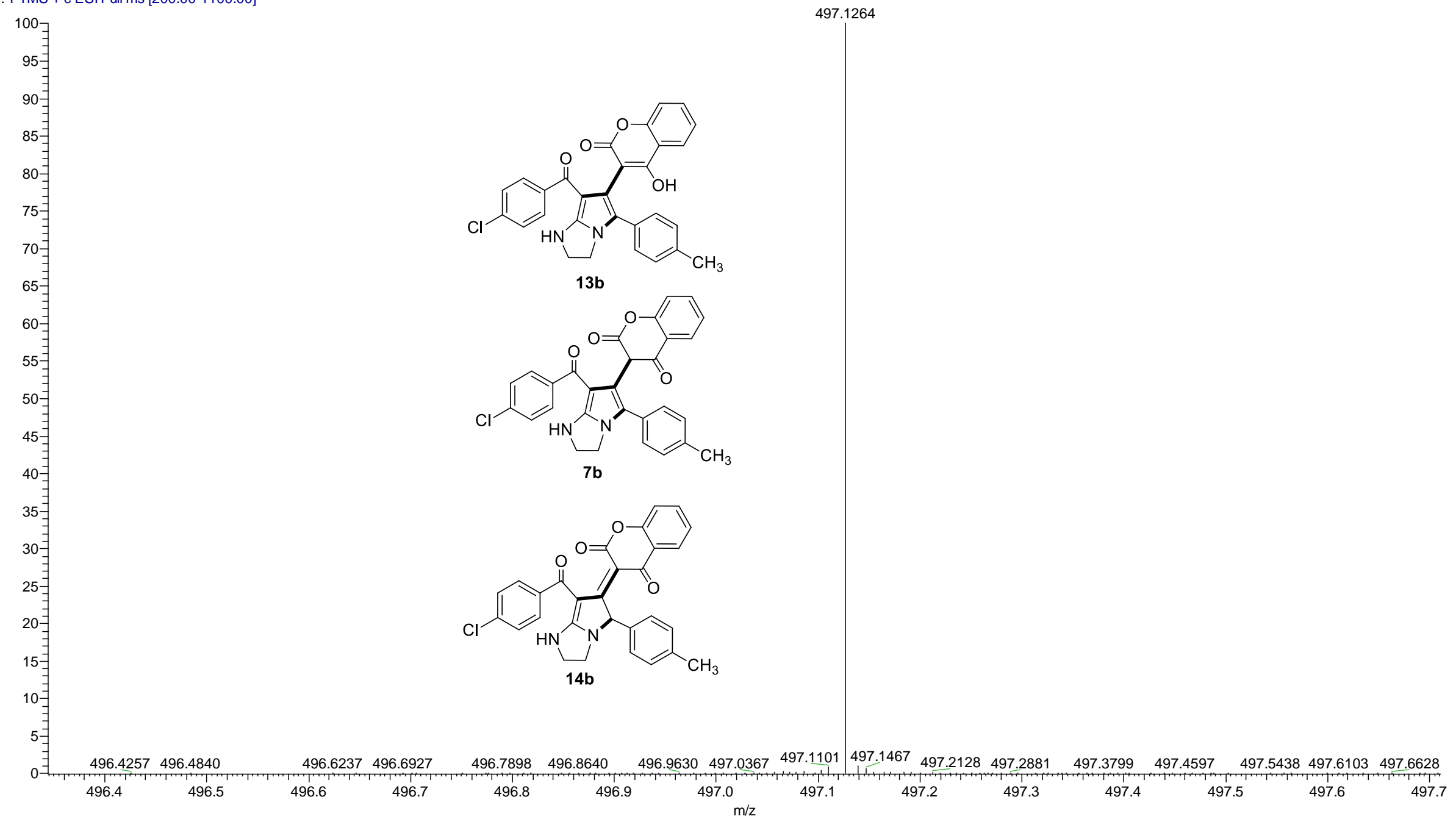

Figure S101. HRMS of intermediate $13 b / 7 b / 14 b$ 
08-08 \#116 RT: 2.28 AV: 1 NL: 3.19E5

T: FTMS + c ESI Full ms [200.00-1100.00]

100

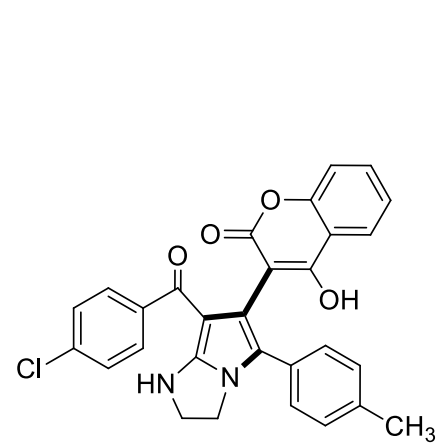

497.1257

$$
\begin{aligned}
& 95 \\
& 90 \\
& 85 \\
& 80 \\
& 75 \\
& 70 \\
& 65 \\
& 60 \\
& 55 \\
& 50 \\
& 45 \\
& 40 \\
& 35 \\
& 30 \\
& 25 \\
& 20 \\
& 20 \\
& 15 \\
& 10 \\
& 5 \\
& 0 \\
& 0
\end{aligned}
$$

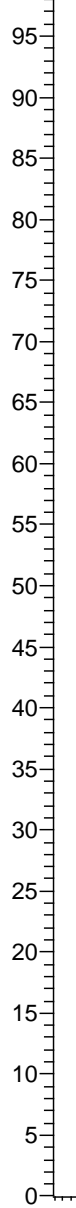

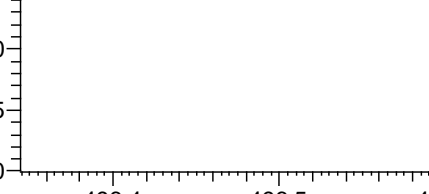

496.5

496.6

Figure S102. HRMS of intermediate 13b/7b/14b 
08-08\#116 RT: 2.28 AV: 1 NL: $3.19 E 5$

T: FTMS + c ESI Full ms [200.00-1100.00]

100 习

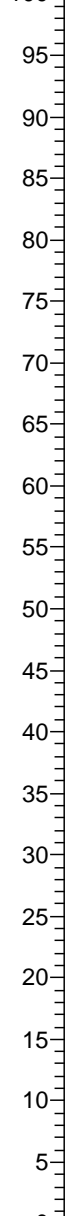

10 每

496.4

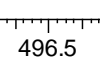

496.6

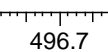

$496.8 \quad 496.9$

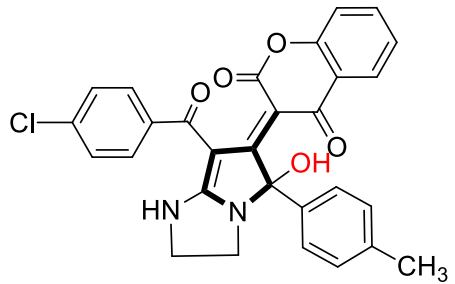

Figure S103. HRMS of compound $6 \mathrm{~b}$

497.4559

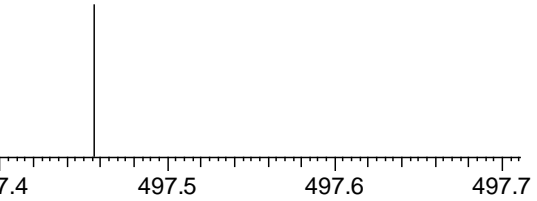




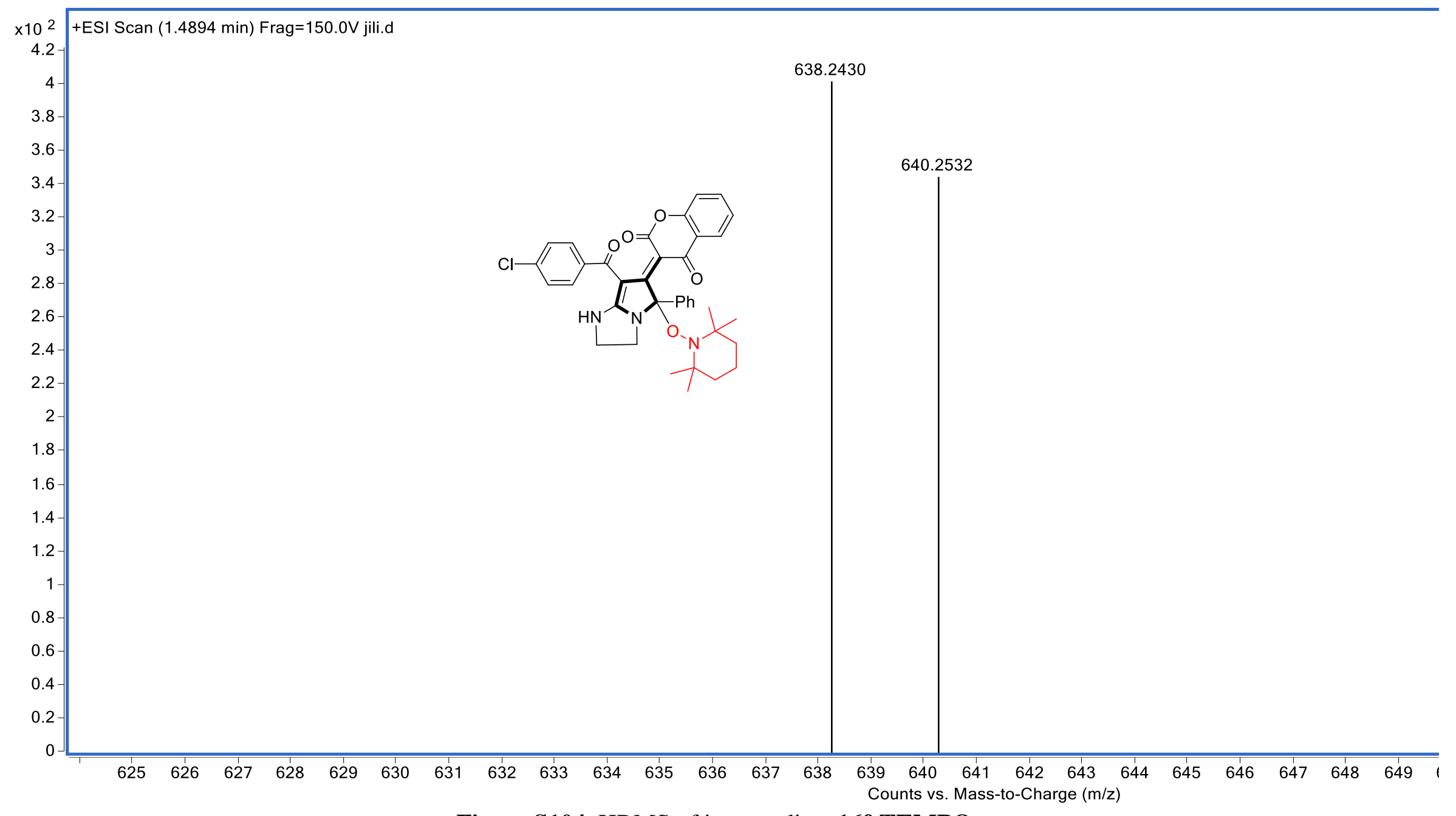

Figure S104. HRMS of intermediate 16f-TEMPO 


\section{References and Notes}

1. (a) Mertens, H.; Troschütz, R. Synthese primärer Nitroketenaminale. Arch. Pharm. 1986, 319, 161-167. (b) Li, K.; Chen, L.; Fan, Y.-X.; Wei, Y.; Yan, S.-J. Multicomponent Tether Catalysis Synthesis of Highly Functionalized 4-(Pyridin-2-ylmethyl)-2-aminopyrroles via Cascade Reaction Is Accompanied by Decarboxylation. J. Org. Chem. 2019, 84, 11971-11982.

2. (a) Huang, Z.-T.; Wang, M.-X. A New Route to 3H-1,5-Benzodiazepines and Heterocylic Ketene Aminals from Benzoyl Substituted Ketene Dithioacetals and Diamines. Synthesis 1992, 12, 1273-1276. (b) Huang, Z.-T.; Liu, Z.-R. Synthesis of 2-(Benzoylmethylene)imidazoldines and -hexahdropyrimidines by Condensation of Ethyl Benzoylacetimidates With 1,2-Ethamediamine or 1,3-Propandiamine, and Some Addition Reactions. Synthesis 1987, 4, 357-362. (c) Huang, Z.-T.; Liu, Z.-R. SYNTHESIS OF BENZOYL SUBSTITUTED HETEROCYCLIC KETENE AMINALS BY CONDENSATION OF BENZOYL KETENE MERCAPTALS WITH DIAMINES. Synth. Commun. 1989, 19, 943-958. (d) Li, Z.-J.; Smith, C. D. THE SYNTHESIS OF FLUOROHETEROCYCLIC KETENE AMINALS. Synth. Commun. 2001, 31, 527-533.

3. CCDC1916446 contain the supplementary crystallographic data for compound 6c. CCDC1916449 contain the supplementary crystallographic data for compound $\mathbf{6 v}$. These data can be obtained free of charge from The Cambridge Crystallographic Data Center via $\underline{\text { www.ccdc.cam.ac.uk/data_request/cif }}$ 